\title{
Control of continuum robots for minimally invasive interventions
}

\section{Gustaaf J. Vrooijink}




\section{CONTROL OF CONTINUUM ROBOTS FOR MINIMALLY INVASIVE INTERVENTIONS}

Gustaaf J. Vrooijink 



\title{
CONTROL OF CONTINUUM ROBOTS FOR MINIMALLY INVASIVE INTERVENTIONS
}

\section{DISSERTATION}

\author{
to obtain \\ the degree of doctor at the University of Twente, \\ on the authority of the rector magnificus, \\ Prof. dr. T.T.M. Palstra, \\ on account of the decision of the Doctorate Board, \\ to be publicly defended \\ on Wednesday the $13^{\text {th }}$ of November 2019 at 16:45 hours
}

\section{Gustaaf Johannes Vrooijink}

born on the $29^{\text {th }}$ of January 1986 in Oldenzaal, the Netherlands 
This dissertation has been approved by:

Prof. dr. Sarthak Misra

Cover design: Gustaaf J. Vrooijink

Printed by: Ipskamp Printing

ISBN: 978-90-365-4888-5

DOI: $\underline{10.3990 / 1.9789036548885}$

(C) 2019 Gustaaf J. Vrooijink, the Netherlands. All rights reserved. No parts of this thesis may be reproduced, stored in a retrieval system or transmitted in any form or by any means without permission of the author. Alle rechten voorbehouden. Niets uit deze uitgave mag worden vermenigvuldigd, in enige vorm of op enige wijze, zonder voorafgaande schriftelijke toestemming van de auteur. 


\section{Graduation Committee}

Chairman/secretary: Prof. dr. ir. G.P.M.R. Dewulf University of Twente

Supervisor:

Prof. dr. S. Misra

University of Twente

\& University Medical

Center Groningen

Committee Members: Prof. dr. N. Simaan

Vanderbilt University

Prof. dr. ir. P. Breedveld

Delft University of

Technology

Prof. dr. J. Dankelman

Delft University of Technology

Prof. dr. ir. H.F.J.M. Koopman

University of Twente

Dr. ir. R.G.K.M. Aarts

University of Twente 

This work is part of the research programme iMIT with project number 12704, which is (partly) financed by the Dutch Research Council (NWO).
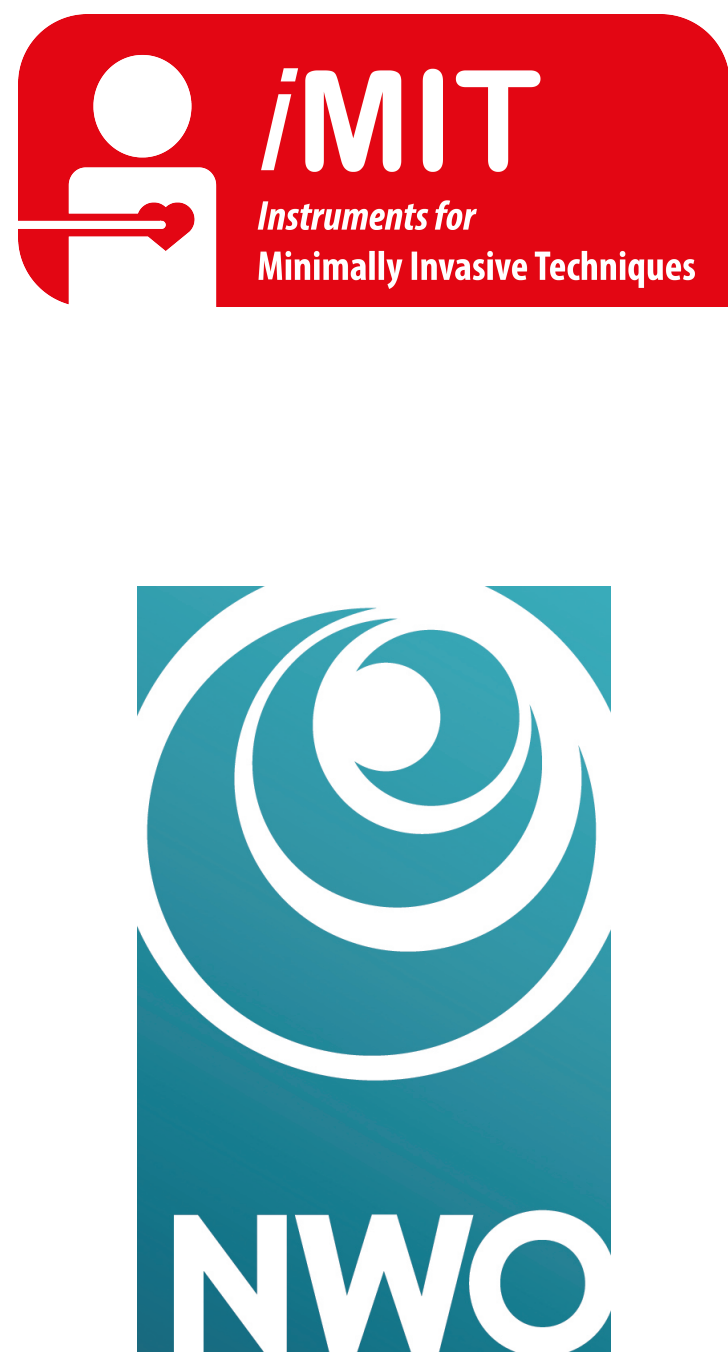


\section{Summary}

In recent times, the role of minimally invasive surgery (MIS) in diagnosis and therapy has significantly increased. The rapid adoption of MIS can be attributed to the considerable benefits offered to patients. This includes reduced patient trauma, less scarring and faster recovery times. Furthermore, the reduced patient trauma enables the treatment of high-risk patients who were initially denied surgery. However, restricted access, reduced visibility and limited dexterity of instruments at the treatment location are among the challenges of MIS. Hence, the demand for new technology such as continuum robots that can assist the clinician during MIS is significant. Potentially, the control of continuum robots such as flexible needles, delivery sheaths and steerable catheters could be used to address challenges associated with MIS.

In this thesis, closed-loop control methods using flexible needles, delivery sheaths and steerable catheters for percutaneous and cardiovascular interventions are developed. The closed-loop control methods are used to accurately position the continuum robot at the treatment location and to provide instrument tip stabilization for tissue motions. Feedback of the continuum robot and target location for closed-loop control is provided by ultrasound images and electromagnetic tracking. The closed-loop control methods (aided by path planning) uses continuum robot models for the accurate positioning and stabilization of the instrument tip. Furthermore, the control input is provided to actuators and mechanisms in order to steer the continuum robot. In order to evaluate the closed-loop control of continuum robots, an experimental testbed is developed and used to reproduce a clinical environment.

The proposed closed-loop control methods for continuum robots are evaluated in clinically-relevant experiments. The results obtained from experiments showed an improved continuum robot tip positioning compared to clinical practice. In order to avoid sensitive tissue that could be present in surgery, it is demonstrated that constraints integrated in the control method could be used to restrict the instrument motion. Furthermore, stabilization of the continuum robot tip for tissue motions is demonstrated. However, the proposed closedloop control method for continuum robots could benefit from improvements in feedback, control methods, continuum robot design, and instrument steering mechanisms with actuators. Therefore, with modifications, the proposed methods could be successfully deployed in clinical practice. 


\section{Samenvatting}

In de afgelopen jaren is de rol van minimaal invasieve chirurgie (MIC) bij diagnose en therapie aanzienlijk toegenomen. De snelle toename van MIC is te danken aan de grote voordelen voor patiënten, zoals minder trauma, kleinere littekens en snellere hersteltijden. Bovendien zorgt de verminderde schade aan het lichaam ervoor, dat patiënten met een hoog risico, waarbij aanvankelijk een operatie niet mogelijk werd geacht, toch behandeld kunnen worden. Echter, een beperkte toegang, verminderd zicht en gereduceerde behendigheid van het instrument op de plek van behandeling behoren tot de uitdagingen van MIC. Hierdoor is de vraag naar nieuwe technologie, zoals continuümrobots, die de clinicus kan helpen tijdens MIC, aanzienlijk. Mogelijk kan de aansturing van continuümrobots, zoals flexibele naalden, stuurbare toegangsschede en katheters gebruikt worden om uitdagingen in MIC te ondervangen.

In dit proefschrift zijn er regeltechnische methoden ontwikkeld voor flexibele naalden, stuurbare toegangsschedes en katheters in percutane en cardiovasculaire interventies. De regelaars worden gebruikt om de continuümrobot nauwkeurig op de behandellocatie te positioneren en om de instrumenttip te stabiliseren tegen beweging van weefsel. Terugkoppeling van de continuümrobot en de doellocatie worden via echografie en elektromagnetische volgsensoren verkregen. Het regelsysteem (met behulp van pad planning) maakt gebruik van de eerder genoemde terugkoppeling en van continuümrobot modellen voor de stabilisatie en positionering van de instrumenttip. Tenslotte wordt het stuursignaal van de regelaar door een bedieningsmechanisme met actuatoren gebruikt om de continuümrobot te kunnen sturen. Om de regelsystemen voor continuümrobots te kunnen evalueren, is er een testopstelling ontwikkeld om de klinische omgeving te reproduceren.

De voorgestelde regeltechnische methoden voor continuümrobots zijn geëvalueerd door middel van klinisch relevante experimenten. De resultaten van deze experimenten laten een verbeterde positionering van de instrumenttip zien ten opzichte van ervaringen uit de praktijk. Daarnaast is er, om sensitief weefsel in de buurt van de behandellocatie te vermijden, een restrictie opgenomen in de regelaar. Deze restrictie heeft aangetoond, dat de beweging van het instrument beperkt wordt, zodat er geen schade aan weefsel kan worden aangericht. Tevens is de stabilisatie van de instrumenttip tegen bewegingen van weefsel aangetoond. Echter, de voorgestelde besturingsmethoden voor continuümrobots kunnen profiteren van verbeteringen in feedback, regelaar, continuümrobot ontwerp en bedieningsmechanisme met actuatoren. Desalniettemin zouden de voorgestelde regeltechnische methoden, met bovenstaande wijzigingen, succesvol toegepast kunnen worden in MIC. 
Contents

Summary

Samenvatting v v

\begin{tabular}{lll}
\hline & Introduction & 3
\end{tabular}

$1.1 \quad$ Clinical applications . . . . . . . . . . . . . . . . . 3

1.2 Challenges and proposed methods . . . . . . . . . . . . . . 8

1.2 .1 Related work . . . . . . . . . . . . . . . . . . . . . 9

1.3 Objectives . . . . . . . . . . . . . . . . . . . 13

1.4 Contributions . . . . . . . . . . . . . . . . . . . . . . . . . . . . . . . . . . . . . .

1.5 Outline of the thesis . . . . . . . . . . . . . . . 16

Part I Continuum robots: Needle steering ___ 17

2 Needle path planning and steering in a three-dimensional non$\begin{array}{ll}\text { static environment using two-dimensional ultrasound images } & 21\end{array}$

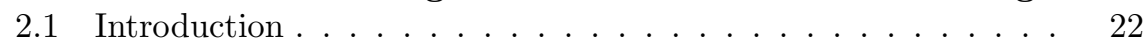

2.2 Related Work . . . . . . . . . . . . . . . . . . . . . 24

2.3 Methods . . . . . . . . . . . . . . . . . . 25

2.3 .1 Ultrasound Image Processing . . . . . . . . . . . . . . . . . . 26

$2.3 .2 \quad$ Needle Tip Pose Estimation . . . . . . . . . . . . . . . . 27

2.3 .3 Ultrasound Image-Guided Controller . . . . . . . . . . . 29

2.3 .4 Motion Planning . . . . . . . . . . . . . . . . . 32

2.3 .5 Duty Cycled Needle Steering . . . . . . . . . . . . . . . . . . . . 35

2.4 Experiments . . . . . . . . . . . . . . . . . . 37

$2.4 .1 \quad$ Experimental Setup and Materials . . . . . . . . . . . . 37

2.4 .2 Experimental Scenarios . . . . . . . . . . . . . . . . . . . . . . . . 39

2.4 .3 Experimental Results . . . . . . . . . . . . . . . . . 40

2.5 Conclusions and Future Work . . . . . . . . . . . . . . . . . . 43

Part II Continuum robots: Catheter steering ___ 45

3 Model predictive control of a robotically-actuated delivery sheath for beating heart compensation 49

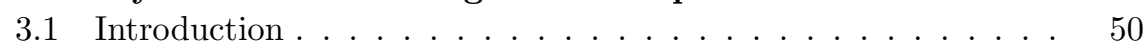

3.1 .1 Related Work . . . . . . . . . . . . . . . . 51 
3.1 .2 Contributions . . . . . . . . . . . . . . . . 52

3.2 Methods . . . . . . . . . . . . . . . . . . . . . 53

$3.2 .1 \quad$ Instrument modeling . . . . . . . . . . . . . 54

3.2.1.1 $\quad$ Forward kinematics . . . . . . . . . . . . . 54

3.2.1.2 Inverse kinematics . . . . . . . . . . . . . . 57

3.2.1.3 Differential kinematics . . . . . . . . . . . . . 58

3.2 .2 Ultrasound image segmentation . . . . . . . . . . . . . . 59

3.2.3 Model predictive control . . . . . . . . . . . . . 63

$3.2 .3 .1 \quad$ Model description . . . . . . . . . . . . . . 64

3.2.3.2 Control objective. . . . . . . . . . . . . . . . 65

3.2.3.3 $\quad$ Aortic heart valve modeling. . . . . . . . . . . 67

3.2.3.4 Controller design . . . . . . . . . . . . . . . . . . . . 68

3.2.3.5 Hysteresis compensation . . . . . . . . . . 72

3.3 Experiments . . . . . . . . . . . . . . . . . . . . . . 72

3.3.1 Experimental setup and materials . . . . . . . . . 72

3.3.2 Experimental scenarios . . . . . . . . . . . . . . . . 74

$3.3 .2 .1 \quad$ Circular path . . . . . . . . . . . . . 74

3.3.2.2 Aortic heart valve motion tracking . . . . . . . 75

3.3.3 Experimental results . . . . . . . . . . . . . . . 75

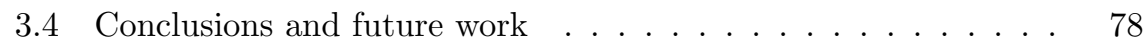

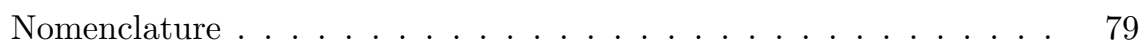

3.A Concept of prediction . . . . . . . . . . . . . . 81

4 Sensor-Guided Stabilization of a Steerable and Flexible Catheter Tip for Beating Heart Mitral Valve Motions 83

$4.1 \quad$ Introduction . . . . . . . . . . . . . . . . . . . . . 85

4.1 .1 Related work . . . . . . . . . . . . . . . . 85

4.1 .2 Contributions . . . . . . . . . . . . . . . . 86

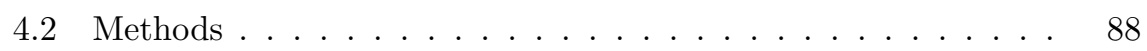

$4.2 .1 \quad$ Experimental setup. . . . . . . . . . . . . . . . 88

4.2.2 $\quad$ Steerable and flexible catheter (SFC) modeling . . . . . 90

$4.2 .3 \quad$ Catheter steering module . . . . . . . . . . . . 92

\begin{tabular}{|lll}
\hline 4.2 .4 & Steerable and flexible catheter tracking and segmentation 96
\end{tabular} 4.2.4.1 $\quad$ Electro-Magnetic tracking. . . . . . . . . . . . . . 97

$4.2 .4 .2 \quad$ Ultrasound segmentation . . . . . . . . . . . . . . . 97

$4.2 .5 \quad$ Sensor-guided controller . . . . . . . . . . . . . . . . . . 99

$4.2 .6 \quad$ Beating heart mitral valve modeling . . . . . . . . . . . 101

4.3 Experiments . . . . . . . . . . . . . . . . . 103

4.3 .1 Experimental plan . . . . . . . . . . . . . . 103

4.3 .2 Results . . . . . . . . . . . . . . . . . . . . 104

4.4 Conclusions and future work . . . . . . . . . . . . . . 106 
\begin{tabular}{|lll|}
\hline 5 & A Beating Heart Testbed for the Evaluation of Robotic Car- \\
\hline & diovascular Interventions & 107 \\
\hline
\end{tabular}

5.1 Introduction . . . . . . . . . . . . . . . . . . . . . 108

5.2 Methods . . . . . . . . . . . . . . . . . . 111

5.2 .1 Experimental testbed . . . . . . . . . . . . 111

$5.2 .2 \quad$ Beating heart mitral valve motion modeling . . . . . . . 113

$5.2 .3 \quad$ Cardiac pump . . . . . . . . . . . . . . 115

5.2 .4 Systemic circulation modeling . . . . . . . . . . . . . . 117

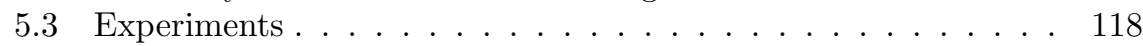

5.3.1 Experimental plan . . . . . . . . . . . . . . . . . . 118

5.3 .2 Results $\ldots \ldots \ldots \ldots$. . . . . . . . . . . . . . . . . . . . . . . . . 118

5.4 Conclusions and future work . . . . . . . . . . . . . 120

Part III Outlook 123

6 Conclusions and future work $\quad \mathbf{1 2 5}$

6.1 Conclusions . . . . . . . . . . . . . . . . . 125

6.2 Future work . . . . . . . . . . . . . . . . . . . 127

$\begin{array}{ll}\text { Bibliography } & 131\end{array}$

$\begin{array}{lr}\text { Acknowledgements } & 149\end{array}$

$\begin{array}{ll}\text { About the author } & 151\end{array}$ 


\section{CHAPTER 1 \\ Introduction}

Over the last decades, medical treatment provided by minimally invasive surgery (MIS) has significantly increased [1-3]. The increased use of MIS can be explained by the advances in medical technology. Treatment provided by MIS offers considerable benefits for patients. This includes reduced trauma and improved quality of life. The reduced patient trauma enables the treatment of high-risk patients who would otherwise be denied surgery. Furthermore, the reduced patient trauma is often associated with shorter hospital stays. Thus, reducing the costs and increasing productivity of healthcare 4].

The use of continuum robots such as steerable and flexible needles, delivery sheaths, endoscopes, laparoscopic devices and catheters can provide a significant contribution to MIS. Continuum robots can be used in MIS to address challenges such as limited dexterity of the instrument at the treatment location, which often impedes successful treatment. The goal of this doctoral research is on the design and control of continuum robotic methods used in a realistic clinical environment. Further, the goal is to use novel multi-steerable catheter technology to enable accurate positioning at a treatment location in order to assist the clinician during the procedure. The closed-loop control strategies using continuum robots provided in this thesis aim to improve the capabilities of MIS in two main research areas. In Part I, the closed-loop control strategies for percutaneous applications using continuum robots (i.e., steerable and flexible needles) are described. In Part II, the closed-loop control strategies using continuum robots such as delivery sheaths and catheters for cardiovascular interventions are presented.

\subsection{Clinical applications}

In this section, medical applications are described that can benefit from the use of continuum robots such as steerable and flexible needles, delivery sheaths and catheters. The scope of the medical applications is limited to percutaneous and cardiovascular procedures. However, other applications in neurosurgery, urological and thoracic interventions could be improved by the image-guided control of continuum robots.

Percutaneous procedures are often performed by instruments such as needles, which are used for the extraction of tissue or to perform therapy. Needle 


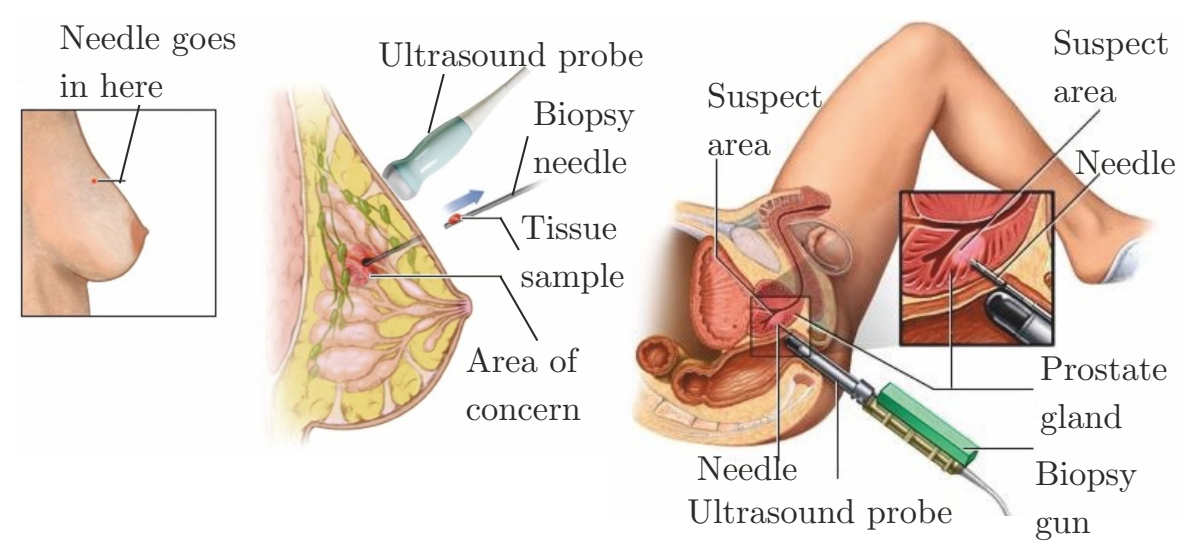

(a)

(b)

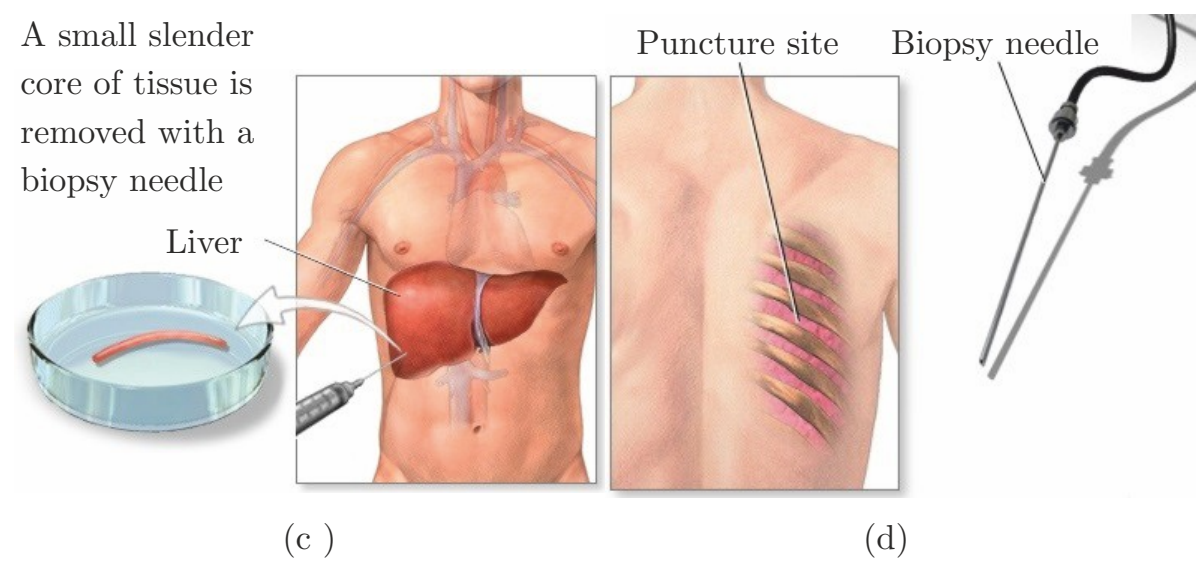

Figure 1.1: Examples of minimally invasive surgical procedures using needles. (a) During a breast biopsy, a needle is used to remove a small piece of tissue sample for diagnostic purposes (image courtesy of Healthwise Incorporate, Boise, USA (C)). (b) A prostate biopsy is performed in order to evaluate if the retrieved tissue is malicious (image courtesy of Mayo Foundation for Medical Education and Research, Rochester, USA (C)). (c) A percutaneous procedure is conducted in order to extract and evaluate liver tissue for signs of damage or disease (image courtesy of Icahn School of Medicine at Mount Sinai, New York City, USA (C)). (d) A lung tissue biopsy is performed in order to evaluate if a lung disease or cancer is present (image courtesy of Icahn School of Medicine at Mount Sinai, New York City, USA (C).

tip placement in biopsy or therapy is often performed using feedback from medical imaging modalities 5,6$]$. Crucial to the success of such percutaneous procedures is the accurate guidance of the instrument tip to reach the target tissue. If needle placement is unsuccessful, the instrument should be retracted and reinserted. Therefore, several attempts may be needed before accurate nee- 


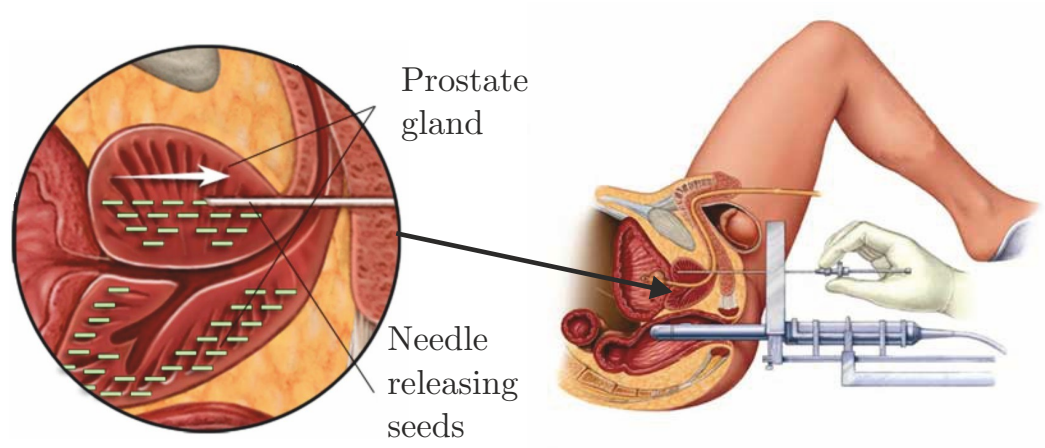

Figure 1.2: A prostate brachytherapy procedure, the needle is used to insert an encapsulated radioactive seeds into the prostate, inside or next to the area requiring treatment (image courtesy of Mayo Foundation for Medical Education and Research, Rochester, USA (C)).

dle positioning is provided. Inaccurate instrument tip placement could result in misdiagnosis during biopsy and unsuccessful treatment of cancer during therapy 7. Further, inaccurate instrument tip placement could result in unwanted tissue damage.

A biopsy is performed in order to extract tissue from breast, prostate, liver and lung for diagnostic purposes as depicted in Fig. 1.1. A breast biopsy is performed in order to extract a tissue samples for diagnosis. In order to visualize both instrument and suspected tissue medical images obtained from ultrasound, magnetic resonance and mammography are used. The needle is often inserted under image guidance in order to retrieve several tissue samples. A prostate biopsy is performed if a prostate-specific antigen blood test or digital rectal exam indicates abnormalities. Using a needle, the urologist extracts multiple tissue samples for diagnosis such as cell abnormalities that could be prostate cancer. In prostate biopsy, the needle is often guided by transrectal ultrasonography or magnetic resonance imaging. A liver biopsy is a procedure to remove a small slender core of liver tissue for examination. The needle is inserted under ultrasound-image guidance for accurate needle placement and to avoid damage to nearby organs. Subsequently, the retrieved tissue samples are analysed under a microscope for signs of damage or disease. A lung biopsy is often considered if abnormalities in the chest are observed during a computerized tomography scan or chest X-ray. Samples of tissue or cells are obtained by core needle biopsy or by fine needle aspiration, respectively. The biopsy needle is guided by computerized tomography scans, fluoroscopy, ultasound or magnetic resonance images to reach the site of the abnormal growth. The retrieved samples are analysed for malignant tissue. In order to provide successful diagnosis, accurate needle placement is required, which is often impeded my respiration. A needle could be retracted and re-inserted to reach the site of the abnormal growth. However, multiple needle insertions increases the 


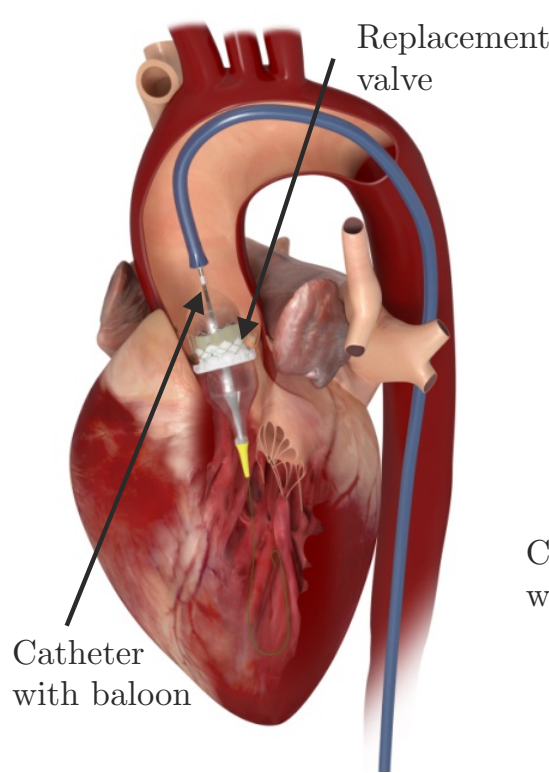

(a) Transfemoral TAVI

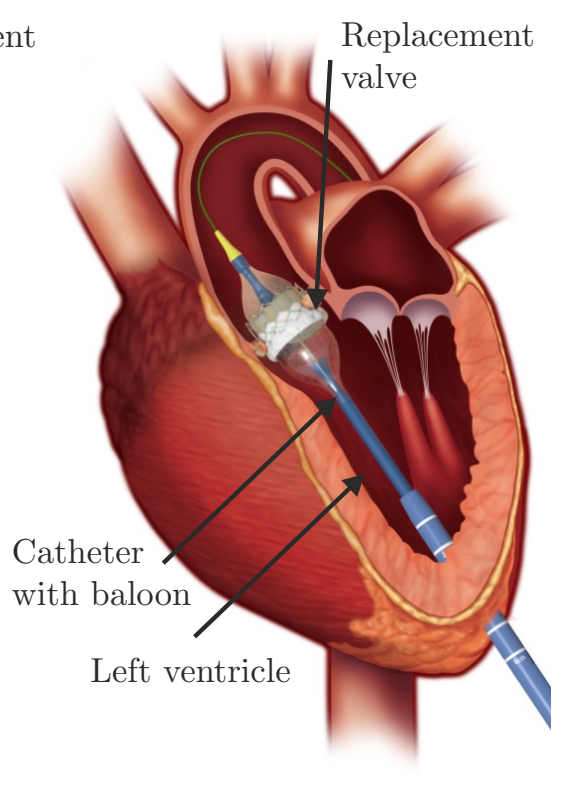

(b) Transapical TAVI

Figure 1.3: A minimally invasive surgical approach for the implantation of a prosthetic aortic valve (image courtesy of Maastricht UMC+, Maastricht, the Netherlands (C)). The method is known as Transcatheter Aortic Valve Implantation (TAVI), which can be employed using the transfemoral route as depicted in (a) or the transapical route shown in (b).

risk of pneumothorax.

Furthermore, therapeutic procedures such as localized drug delivery, cryotherapy and brachytherapy are performed in order to provide treatment. Brachytherapy is performed by implanting radioactive sources in order to destroy cancer cells as demonstrated in fig. 1.2. The radioactive sources are used to deliver a high doses of radiation to a specific area in the prostate gland compared to conventional external beam radiation. The radioactive sources are implanted in the prostate gland using a needle, which is guided guided by medical imaging modalities such as ultrasound. If placement of the radioactive sources is inaccurate, the dose provided to the intended cancer cells may not be sufficient, which could require additional treatment.

Cardiovascular diseases related to heart valves such as symptomatic aortic stenosis require treatment. This can be provided by open heart aortic valve replacement with cardiopulmonary bypass. However, open heart aortic valve replacement is often considered a high-risk procedure for elderly with comorbidities $1,8,9$. As an alternative, a minimally-invasive method for the treatment of aortic stenosis is provided by transfemoral (TF) or transapical (TA) transcatheter aortic valve implantation (TAVI) as depicted in Fig. 1.3 [1] 3 . 


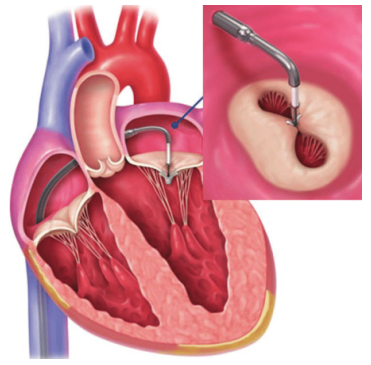

(a) MitraClip placement. Inset shows the atrial view.

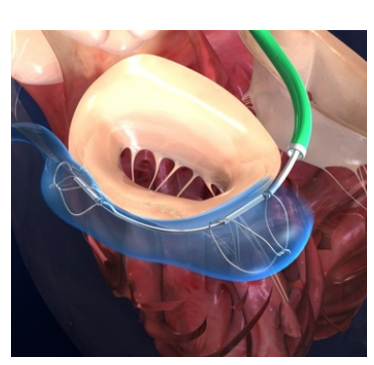

(b) Indirect mitral annuloplasty.

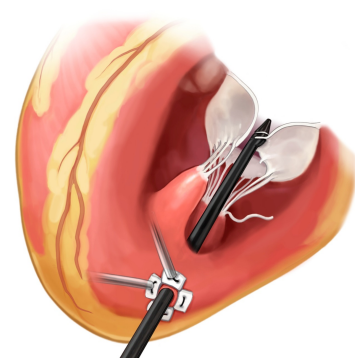

(c) Artificial chordae implantation.

Figure 1.4: Examples of minimally invasive surgical procedures for the treatment of degenerative mitral valve deceases. (a) A MitraClip is placed on the mitral valve leaflets in order to provide treatment for severe (commissural) mitral regurgitation (image courtesy of Abbott Laboratories, Chicago, USA (C)). (b) Indirect mitral annuloplasty is performed to treat severe mitral regurgitation caused by mitral annular enlargement. Indirect annuloplasty is performed by using an implantable mitral annular contour system, which is placed into the coronary sinus and great cardiac vein (image courtesy of Cardiac Dimensions Pty. Ltd. and subsidiaries, Sydney, Australia (C)). (c) Treatment for mitral valve leaflet prolapse caused by a damaged or ruptured chordae tendinae is provided by implanting an artificial chordae using a transapical approach (image courtesy of NeoChord, Inc., St. Louis Park, USA (C)).

TAVI performed using the transapical approach provides a minimally-invasive method with direct surgical access through the apex of the heart for the replacement of the aortic valve. In TA-TAVI procedures, complications such as prosthetic valve malpositioning have been reported 10 . The malpositioning and deployment of the prosthetic aortic valve implant could result in periprosthetic aortic regurgitation, valve embolization and occlusion of arteries. Addressing the malpositioning prosthetic valve often requires conversion to surgical aortic valve replacement with cardiopulmonary bypass or a valve-invalve procedure 11. Hence, the success of the procedure is highly dependent on the accurate prosthetic valve orientation, positioning and deployment.

Similar to aortic valve replacement, open heart surgery via sternotomy is used to enable repair strategies in order to provide treatment to a degenerative mitral valve disease. The aim of a repair strategy is to restore the original mitral valve function and to preserve the natural hemodynamics. Further, repair strategies reduce the risk of embolism and minimize the need for long-term anticoagulation, which is beneficial for the quality of life of the patient [12,13]. Although, superior surgical access to the deceased mitral valve is provided by open heart surgery via sternotomy, the procedure is associated with severe patient trauma. Thus, high-risk patients with comorbidities are often excluded from surgery [14]. As an alternative, limited minimally-invasive methods for mitral valve repair are available $15-18$. Minimally-invasive methods without cardiopulmonary bypass are often impeded by beating-heart motions and re- 
quires the attention of the clinician, whose accuracy deteriorates for complex repetitive motions up to 60 beats per minute 19 . Hence, technology is required that enables and assists the clinician to perform minimally-invasive mitral valve repair.

A number of MIS mitral valve repair strategies are presented in Fig. 1.4. MitraClip therapy is a repair strategy without cardiopulmonary bypass for high risk patients with severe (commissural) mitral regurgitation. The therapy uses a MitralClip to grasp and clamp the mitral valve leaflets, which results in a double orifice of the mitral valve. Ultrasound imaging is used to evaluate MitraClip placement by considering the mitral valve efficiency and the leaflet capture. The MitraClip can be repositioned in order to find the optimal reduction of mitral regurgitation. The Carillon Mitral Contour System provides indirect annuloplasty for high risk patients with severe mitral regurgitation caused by mitral annular enlargement. The implantable mitral annular constraint device is used to improve the function of the mitral apparatus from the venous anatomy of the heart (i.e., coronary sinus and great cardiac vein). The mitral annular constraint device uses distal and proximal anchors connected by a shaping ribbon in order to reshape the mitral valve annulus. The NeoChord artificial chordae delivery system is used to provide repair treatment for high risk patients with severe mitral regurgitation caused by an elongated or ruptured native chordae tendinae. The artificial chordae is implanted using a transapical approach under ultrasound image guidance. The mitral valve leaflet is grasped by the delivery system, while multiple artificial chordae are placed to re-suspend the prolapsed segment.

\subsection{Challenges and proposed methods}

In the majority of the medical interventions, the MIS approach is considered to be a challenge. One of the major challenges in MIS is the reduced visibility at the treatment location, which often impedes the proficient execution of the procedure 18]. Another considerable challenge is the limited dexterity of the instrument at the treatment location. Dexterity of the instrument at locations that are often considered to be confined is necessary for the adequate treatment of the disease. In percutaneous needle interventions, several retractions and reinsertions may be needed before accurate needle placement is provided. Inaccurate needle tip placement could result in misdiagnosis, unwanted tissue damage, complications and unsuccessful treatment. Furthermore, in cardiovascular interventions, the absence of cardiopulmonary bypass requires the clinician to cope with the beating heart motions [19]. The aforementioned challenges pose significant constraints on the capabilities of both instrument and clinician.

In this thesis, sensor-guided control strategies for continuum robots such as steerable and flexible needles, delivery sheaths and catheters are proposed in 
order to address challenges in MIS such as limited visibility, dexterity and tissue motions. By sensor-guided control, the continuum robot could be positioned at the treatment location with accuracy and stabilized for tissue motions. By considering percutaneous procedures, methods for needle placement using closedloop control updated by path planning are proposed. Furthermore, methods for cardiovascular applications are proposed. This includes the stabilization of an instrument for beating heart motions. The instrument tip stabilization for beating heart motions could provide a virtually-still treatment location, which enables the clinician to perform surgery as if the heart stopped beating. Further, the proposed continuum robotic steering methods are evaluated in clinical relevant experimental testbeds.

\subsubsection{Related work}

Tangible improvements in MIS are offered by technological advances in continuum robotics such as steerable and flexible needles, delivery sheaths, endoscopes, laparoscopic devices and catheters, which are often assisted by sensorguided control [20]. By considering percutaneous MIS, improvements have been provided by continuum robots such as steerable and flexible needles 21]. Unlike traditional rigid needles that are restricted to move along a straight path from the needle entry location to the clinical target, flexible needles with an asymmetric (e.g., bevel) tip enable controlled insertion along curved paths 22 27]. The asymmetric forces distributed at the needle tip during insertion enable the needle to deflect along a curved path. For bevel-tipped needles, the deflection occurs in the direction of the bevel tip 28 30]. Further, by orienting the needle during the insertion, the direction of deflection can be controlled, which allows for the needle to be steered towards an intended treatment location 31]. Hence, improved targeting accuracy is offered by continuous adjustments of the needle trajectory 3235 .

Some of the needle concepts described in literature are depicted in Fig. 1.5 . An actuated curved stylet needle (Fig. 1.5(a) is used to automatically steer towards a target 23]. The needle is steered by retracting and extending the curved stylet from the cannula in order to move straight or along a curved path, respectively. The curved stylet needle steering method is developed for percutaneous interventions such as biopsy and localized drug delivery. A programmable bevel concept (Fig. 1.5(b) inspired by a wasp is used to steer the needle towards a target [26]. The needle comprises of four bevel-tipped segments that are interlocked and controlled in order to steer the needle in a desirable direction. The bend radius and direction is controlled by the offset between needle segments. The envisioned applications of the programmable bevel needle concept are keyhole neurosurgery, tissue biopsy and drainage of liver abscesses. An actuated conical tip (Fig. 1.5(c) is used to steer the needle to in a desired direction [36]. The flexible needle with conical tip mounted on a ball joint is controlled by four tendons. Fiber Bragg Grating sensors integrated 


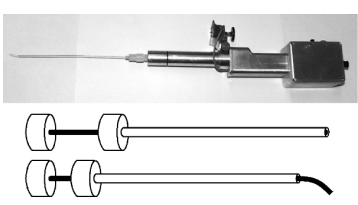

(a)

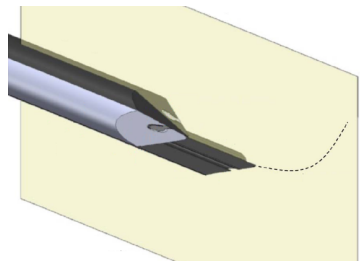

(b)

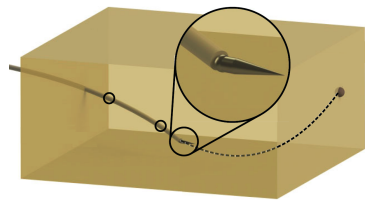

(c)

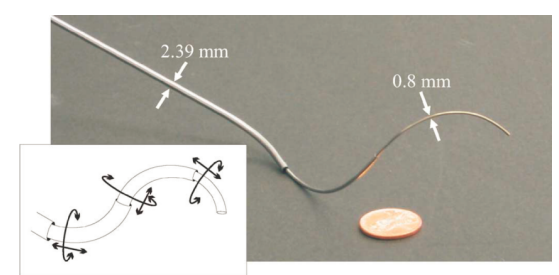

(d)

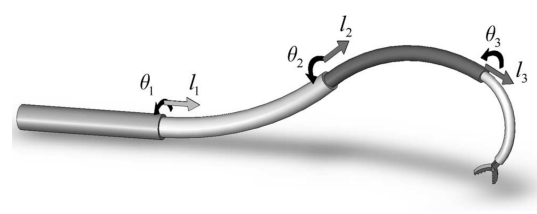

(e)

Figure 1.5: Examples of steerable needle concepts. (a) Curved stylet needle-steering concept: Showing the stylet withdrawn and extended 23. (image courtesy of Okazawa et al., (C) 2005 IEEE). (b) A programmable bevel needle concept inspired by a wasp [26] (image courtesy of Ko et al., (C) 2011 IEEE). (c) An actuated needle concept with a controllable conical tip 36 (image courtesy of Roesthuis et al., (C) 2015 IEEE). An alternative concept is the concentric-tube robot. The concentric-tube robot is capable of puncturing, while dexterity is offered. Concentric-tube robot designs are provided in (d) by Webster III et al. (image courtesy of Webster III et al., (C) 2009 IEEE) 37] and in (e) by Dupont et al. (image courtesy of Dupont et al., (C) 2010 IEEE) 27.

along the needle shaft provide shape reconstruction, which is used in a steering algorithm. The needle with actuated conical tip could potentially be used in applications such as tissue biopsy and brachytherapy.

Alternative concepts such as the concentric-tube robots (Fig. 1.5(d) and Fig. 1.5(e) combine the puncturing capabilities provided by needles and the dexterity offered by catheters 27,37 . The robot is based on a concentric combination of precurved elastic tubes (e.g., nitinol). The individual tubes can be independently translated axially and rotated at the base, which enables the concentric-tube robot to reach a desired treatment location. Potential medical applications of the concentric-tube robots are fetal surgery, cardiovascular surgery, transnasal skull base surgery and transgastric surgery. During insertion, medical imaging modalities such as Ultrasound (US), MagneticResonance (MR), Computed Tomography (CT) and fluoroscopy can be used for feedback of both instrument and treatment location 38 45. The feedback can be used as an input in motion and path planning algorithms in order to compute feasible paths of the needle, which allows for obstacle avoidance and minimization of tissue damage 46 53. The combination of steerable and flexible needles with path planning updated by images obtained from medical 


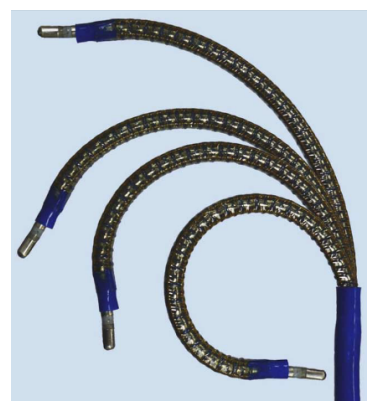

(a)

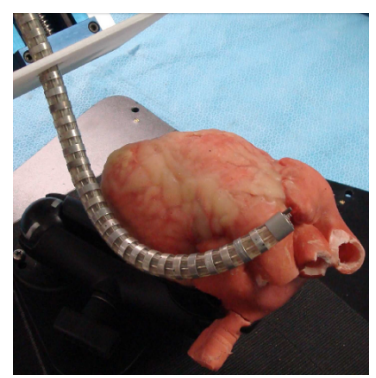

(d)

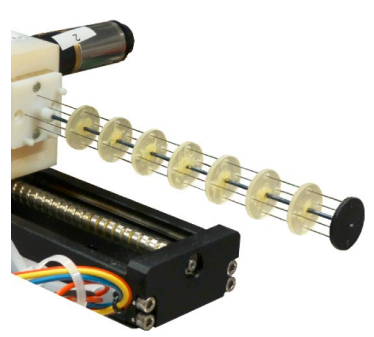

(b)

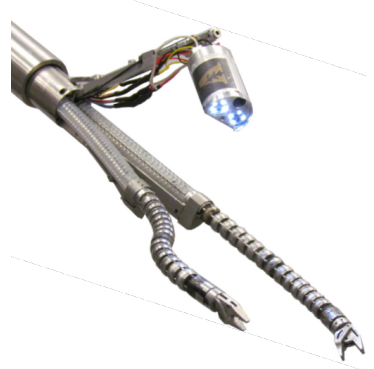

(e)

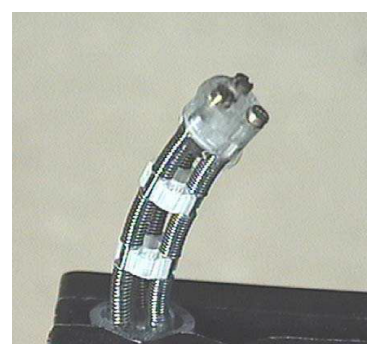

(c)

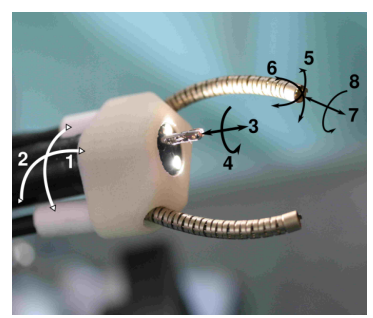

(f)

Figure 1.6: Examples of continuum robot concepts for medical interventions (a) Example of a tendon driven catheter (image courtesy of Camarillo et al., (C) 2008 IEEE) 58]. (b) Example of a tendon driven catheter with integrated shape sensing sensors (image courtesy of Roesthuis et al., (C) 2013 IEEE) [59]. (c) Example of a hydraulically actuated multi active link catheter (image courtesy of Bailly et al., (C) 2005 IEEE) 60. (d) Example of a highly articulated surgical snake robot (image courtesy of Tully et al., (C) 2011 IEEE) 61]. (e) Example of a platform for single port access surgery, which comprises of a 3D stereo vision module and two dexterous arms (image courtesy of Simaan et al., (C) 2013 IEEE) 62]. (f) Example of a robotic platform which comprises of a video endoscope and two articulated arms (image courtesy of Bardou et al., (C) 2010 IEEE) 63.

imaging modalities, can be considered a significant step forward in the development of MIS capabilities 5357 .

Similar to steerable and flexible needles, continuum robots such as catheters have provided contributions to MIS 17, 18]. Continuum robotic catheters are capable of improving existing and enabling future MIS procedures. A number of the continuum robotic catheter concepts documented in literature are presented in Fig. 1.6. The tendon-driven continuum manipulator (Fig. 1.6(a)) modelled by Camerillo et al. is capable of articulating motions in order to provide dexterity 58 . The catheter is actuated by four tendons that are equally spaced on the manipulator perimeter. Further, the catheter inner and outer diameters are lined with laminated plastics for the use in medical applications. 
The tendon-driven continuum manipulator could potentially be used for medical applications in cardiovascular surgery. A tendon-driven manipulator with integrated shape sensing (Fig. 1.6(b) is documented by Roesthuis et al. [59]. A total of twelve Fiber Bragg Grating sensors are distributed over four fibers that are embedded on the flexible nitinol backbone of the manipulator. The strain measurements provided by the Fiber Bragg Grating sensors are used for 3D shape reconstruction. The reconstructed shape of the manipulator is used in closed-loop control. The tendon-driven manipulator could potentially be used to target difficult-to-reach locations in the human body. A hydraulically actuated multi active link catheter (Fig. 1.6(c) is presented by Bailly et al. 60]. The catheter comprises of two bases that are interconnected by equally spaced bellows. The catheter is bend by hydraulic pressure variations in the individual bellows. The hydraulically-actuated catheter is developed for endovascular aortic aneurysm treatment. Further, a highly articulated surgical snake robot (Fig. 1.6(d) is described by Tully et al. 61,64. Although not considered a catheter, the snake robot provides stability and maneuverability by using six actuators. The snake robot is steered towards a location while maintaining its shape in $3 \mathrm{D}$ space. This is achieved by each individual link that moves into the configuration of the link in front of it. Note, that the articulated design maintains it shape in 3D, while instantaneous change of the bend angle requires planning to move from one configuration to another. The highly articulated surgical snake robot is developed for applications such as cell transplantation by intramyocardial injection, epicardial ablation and epicardial lead placement for resynchronization. An insertable robot end effectors platform (Fig. 1.6(e)p is described by Ding et al. and Simaan et al. 62, 65, 66. The insertable robot comprises of two dexterous arms and a 3D stereo vision module, which are controlled by 21 actuators. The insertable robot end effectors platform can be folded into a configuration with a diameter of $15 \mathrm{~mm}$, which allows for platform insertion through a trocar or natural orifice. Further, the robot is designed to perform tasks such as intracorporeal suturing and knot tying. The insertable robot end effectors platform is developed for medical applications in single port access surgery and natural orifice trans-luminal endoscopic surgery. Note, that the insertable robot end effectors platform arms are considered stiffer than a catheter, while the backbones are controlled by push-and-pull actuation. Bardou et al. presented a multiple sections flexible endoscopic system (Fig. 1.6(f)p in order to improve endoscopic tasks and workspace 63. The system comprises of a main endoscope with endoscopic camera and two flexible hollow arms. The arms are attached to the endoscope and provide additional degrees of freedom and triangulation of the instruments which can translate and rotate inside each arm. The multiple sections flexible endoscopic system is developed for medical interventions in natural orifice trans-luminal endoscopic surgery. Further, the kinematics of the aforementioned continuum robotic catheter designs have been documented in literature $27,31,58,63,65,67,68$. The continuum robotic designs offer dexterity, enable clinically relevant tasks such as suturing and 


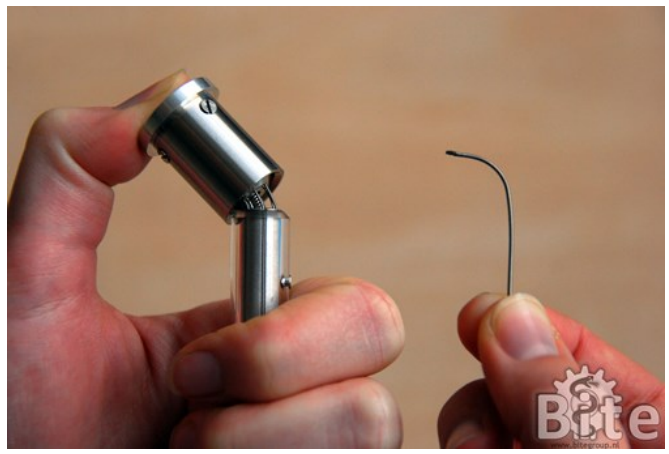

Figure 1.7: Manipulation of a minimally-invasive instrument (i.e., steerable guidewire) for potential use in minimally-invasive surgery. The depicted device has been developed by DEAM Corporation, Amsterdam, the Netherlands in collaboration with the Bio-Inspired TEchnology (BITE) research group of Delft University of Technology, Delft, the Netherlands (image courtesy of the BITE research group of Delft University of Technology, Delft, the Netherlands (C)).

provide improved targeting accuracy, while patient trauma is minimized. In order to provide intuitive control of the continuum robotic catheter, advanced control strategies are combined with catheter steering mechanisms and actuators 69]. A well investigated topic for the use of continuum robotic catheters is the compensation of tissue motion $70-72$. Often predictive control strategies are used in order to address the repetitive respiration and the beating heart motions 73 76]. Another investigated topic is the interaction between catheter tip and tissue using force sensors, which is documented in literature 77, 78. In the aforementioned works, the continuum robotic catheter methods are often assisted by feedback from medical imaging devices such as US, MR, CT or fluoroscopy [2, 32, 39, 41, 44,79. By combining continuum robotic catheters with advanced control strategies aided by feedback from medical images, intuitive and accurate instrument tip positioning can be provided, which can be considered a major step forward in MIS procedures $44,69,80,81$.

\section{$1.3 \quad$ Objectives}

The research provided in the thesis is conducted within the Instrument for Minimally Invasive Techniques (iMIT) program under the project multi steerable catheters (MULTI), which aims to develop and control continuum robots for minimally-invasive interventions 82,83 . The objective of the iMIT program is to enable clinicians to reach, evaluate and treat any location within the human body using a single instrument as depicted in Fig. 1.7. As part of the iMIT program, the MULTI project goal is the development and the closed-loop control of continuum robots in a realistic clinical environment. 
Minimally-invasive surgery on the beating heart is one of the strong drives behind catheter development. Coronary artery balloon dilations form a golden standard, but complex cardiac catheter interventions are still rare. Main difficulties are the absence of vessel wall support, making it hard to position the catheter tip precisely, and the complex $3 \mathrm{D}$ shape of the ventricles requiring intricate catheter maneuvers that are impossible to carry out with existing designs. The added effect of blood flow and tissue motion caused by respiration and heartbeat makes it hard to use catheters for complex interventions such as mitral valve annuloplasty procedures, cardiac tissue resection, atrial septal defect closures and precision cardiac biopsies and transcatheter aortic-valve implantation (TAVI). The aim of the MULTI project is to develop novel multisteerable catheter technology to enable accurate positioning within a beating heart under electromechanically-assisted manual control by a clinician.

\subsection{Contributions}

The main contributions of this thesis are provided by:

- The development of an ultrasound-guided needle insertion method assisted by continuous path planning, which is capable of obstacle avoidance in order to reach a moving target. The integration and testing of the path planning algorithm was achieved in collaboration with the Computational Robotics Research Group in North Caroline University at Chapel Hill, USA.

- The development of a model predictive control strategy used to stabilize the robotically-actuated delivery sheath tip for beating-heart motions, which is based on pre-operative patient data.

- The design of a catheter steering module capable of robot actuation of a wide range of tendon-driven catheters such as a steerable and flexible catheter.

- Presenting the electro-magnetic and ultrasound guided stabilization methods of a steerable and flexible catheter tip for beating heart mitral valve motions.

- The development of a beating heart model with a realistic interior, which is capable of mimicking beating heart motions and systemic circulation in order to provide a realistic cardiac environment for minimally invasive mitral valve repair surgery using steerable and flexible catheters.

Within the context of the thesis, the following articles are published or under consideration in the international peer-reviewed journals: 
- G.J. Vrooijink, M. Abayazid, S. Patil, R. Alterovitz, and S. Misra, Needle path planning and steering in a three-dimensional non-static environment using two-dimensional ultrasound images, International Journal of Robotics Research, 33(10): 1361-1374, September 2014.

- M. Abayazid, G.J. Vrooijink, S. Patil, R. Alterovitz, and S. Misra, Experimental evaluation of ultrasound-guided 3D needle steering in biological tissue, International Journal of Computer Assisted Radiology and Surgery, 9(6): 931-939, November 2014.

- G.J. Vrooijink, A. Denasi, J.G. Grandjean, and S. Misra, Model predictive control of a robotically-actuated delivery sheath for beating heart compensation, International Journal of Robotics Research, 36(2): 193-209, April 2017.

- G.J. Vrooijink and S. Misra, Sensor-Guided Stabilization of a Steerable and Flexible Catheter Tip for Beating Heart Mitral Valve Motions, IEEE Transactions on Medical Robotics and Bionics, 2019 (under review).

The following papers were published at international peer-reviewed conferences:

- G.J. Vrooijink, T.T.M. Ellenbroek, P. Breedveld, J.G. Grandjean, and S. Misra, A preliminary study on using a robotically-actuated delivery sheath (RADS) for transapical aortic valve implantation, in Proceedings of the IEEE International Conference on Robotics and Automation (ICRA), Hong Kong, China, pages 4380-4386, May-June 2014.

- G.J. Vrooijink, M.P. Jansen, M.L. Tolhuisen, J.G. Grandjean, and S. Misra, Ultrasound-guided stabilization of a robotically-actuated delivery sheath (RADS) for beating heart mitral valve motions, in Proceedings of the IEEE International Conference on Biomedical Robotics and Biomechatronics (BioRob), Singapore, pages 73-79, June 2016. (Best Conference Paper Award).

- G.J. Vrooijink, H. Irzan, and S. Misra, A beating heart testbed for the evaluation of robotic cardiovascular interventions, in Proceedings of the IEEE International Conference on Biomedical Robotics and Biomechatronics (BioRob), Enschede, the Netherlands, pages 1076-1082, August 2018.

The following abstract submission was published at a local conference:

- G.J. Vrooijink, J.G. Grandjean, and S. Misra, Closed-loop control of a robotically-actuated delivery sheath (RADS) for cardiovascular applications, in Proceedings of the of the 5th Dutch Biomedical Engineering Conference, Egmond aan Zee, The Netherlands, January 2015. 


\subsection{Outline of the thesis}

The thesis is divided into three parts. The chapters of Part I and II are provided by the aforementioned papers, which are published or under consideration in international peer-reviewed journals and conferences. Part I, II and III of this thesis are outlined as follows:

In Chapter 2 (Part I), the work describing robotic needle interventions for minimally-invasive surgery is presented. A system capable of autonomously and accurately guiding a steerable needle using path planning and ultrasound feedback is provided. A rapidly exploring random tree-based motion planner is used to continuously compute a feasible needle path in order to reach a target. In experiments, the needle is steered around moving obstacles towards a moving target.

In Part II, the sensor-guided control methods of continuum robots for minimally-invasive cardiovascular interventions are provided. The control methods described in Part II consider continuum robots such as a roboticallyactuated delivery sheath and a steerable and flexible catheter. Part II consists of three chapters.

In Chapter 3 (Part II), a model-predictive control (MPC) strategy is presented, which is used to compensate the robotically-actuated delivery sheath (RADS) tip for beating heart motions at a treatment location. Further, kinematic models and on-line ultrasound segmentation methods of the RADS are provided and integrated in the MPC strategy.

In Chapter 4 (Part II), electro-magnetic and ultrasound guided stabilization methods of a steerable and flexible catheter (SFC) tip for beating heart mitral valve (MV) motions are provided. A novel catheter steering module capable of robot actuation of a wide range of tendon-driven catheters is developed. The SFC tip stabilization methods are demonstrated in a heart system, which is attached to a six degrees-of-freedom Stewart platform in order to reproduce beating heart MV motions based on pre-operative patient data.

In Chapter 5 (Part II), a beating heart testbed for the evaluation of robotic catheters in minimally invasive cardiovascular interventions is provided. Aspects of the beating heart testbed such as the relevant interior structures, an integrated realistic MV model, an artificial aortic valve, a systemic arterial model, a venous reservoir and a pulsatile pump are described. Experiments are provided in order to demonstrate systemic circulation and beating heart motion re-production, while a robotic catheter in the heart model is observed by ultrasound imaging and electro-magnetic position tracking.

Finally, in Part III of this thesis, the outlook is provided. In Chapter 6 (Part III) conclusions are summarized and potential directions for future work are given. 


\section{Part I}

\section{Continuum robots: Needle steering}





\section{Preface}

\section{Continuum robots: Needle steering}

In numerous percutaneous procedures, medical instruments are inserted into tissue for diagnosis and therapy. Inaccurate instrument tip placement could result in misdiagnosis and delayed or unsuccessful treatment. Continuum robots such as steerable needles could provide accurate instrument tip positioning. The trajectory of the steerable needle can be adjusted during the procedure in order to reach a moving target with accuracy. In Chapter 2, a closed-loop control strategy for percutaneous applications using steerable needles is provided. An ultrasound tracking method for a steerable needle is developed. The ultrasound feedback of the steerable needle is provided to a path planner. The path planner is used to compute a feasible needle path to the target. Subsequently, the path is provided as an input to closed-loop control of the steerable needle. Furthermore, the ultrasound feedback is used to control the needle in closed-loop. In order to evaluate the proposed needle steering method, experiments are conducted. The aim of the experiments is to demonstrate accurate needle placement for clinically-relevant scenarios with movement in obstacle and target locations. 
CHAPTER 2

Needle path planning and steering in a three-dimensional non-static environment using two-dimensional ultrasound images

\begin{abstract}
Needle insertion is commonly performed in minimally invasive medical procedures such as biopsy and radiation cancer treatment. During such procedures, accurate needle tip placement is critical for correct diagnosis or successful treatment. Accurate placement of the needle tip inside tissue is challenging, especially when the target moves and anatomical obstacles must be avoided. We develop a needle steering system capable of autonomously and accurately guiding a steerable needle using twodimensional (2D) ultrasound images. The needle is steered to a moving target while avoiding moving obstacles in a three-dimensional (3D) non-static environment. Using a 2D ultrasound imaging device, our system accurately tracks the needle tip motion in 3D space in order to estimate the tip pose. The needle tip pose is used by a rapidly exploring random tree-based motion planner to compute a feasible needle path to the target. The motion planner is sufficiently fast such that replanning can be performed repeatedly in a closed-loop manner. This enables the system to correct for perturbations in needle motion, and movement in obstacle and target locations. Our needle steering experiments in a soft-tissue phantom achieves maximum targeting errors of $0.86 \pm 0.35 \mathrm{~mm}$ (without obstacles) and $2.16 \pm 0.88 \mathrm{~mm}$ (with a moving obstacle).
\end{abstract}

This chapter has been previously published as

57] G.J.Vrooijink, M. Abayazid, S. Patil, R. Alterovitz, and S. Misra, Needle path planning and steering in a three-dimensional non-static environment using two-dimensional ultrasound images, International Journal of Robotics Research, 33(10): 1361-1374, September 2014. 
Chapter 2. Needle path planning and steering in a three-dimensional non-static environment using two-dimensional ultrasound images

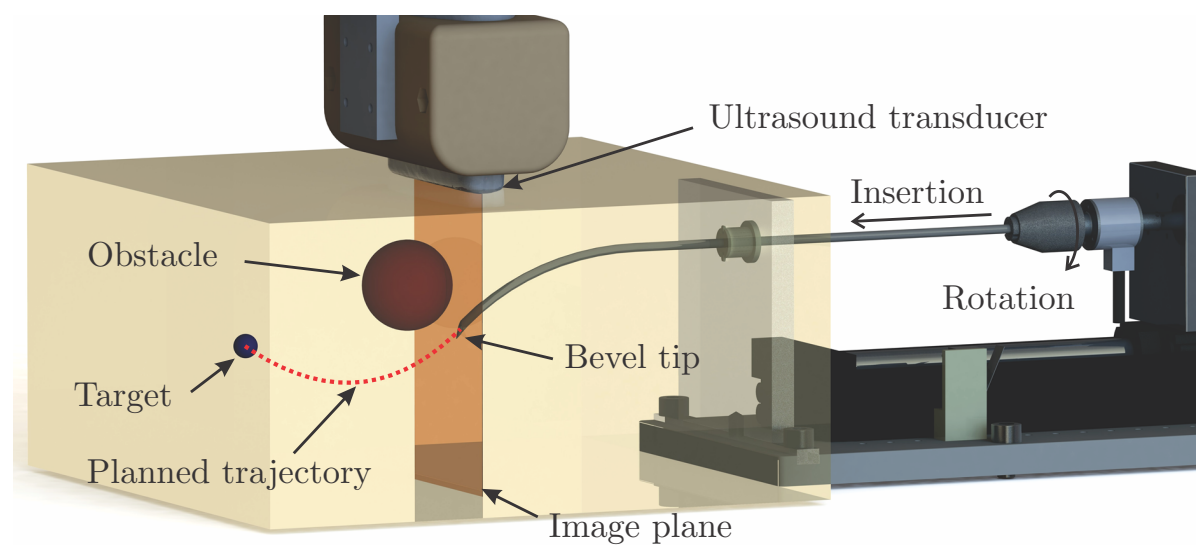

Figure 2.1: A flexible bevel-tipped needle is steered in the soft-tissue phantom by using a device that robotically inserts and rotates the needle. The needle deflects along a curved trajectory in the direction of the bevel tip. A two-dimensional ultrasound transducer, which is orientated perpendicular to the needle insertion direction, is used to track the needle tip in three-dimensional space during insertion. A transducer positioning device is used to track the needle tip during insertion in order to estimate the needle tip pose. The needle tip pose is used to plan and control the needle motion to reach a moving target while avoiding possibly moving obstacles.

\subsection{Introduction}

Percutaneous needle insertion into soft tissue is a component of many minimally invasive medical procedures. Percutaneous needles are used for diagnostic and therapeutic procedures, including biopsy to extract tissue samples for diagnosis and brachytherapy for implanting radioactive sources into tumors for cancer treatment. These procedures are typically performed under image guidance using imaging modalities such as computed tomography (CT), magnetic resonance (MR), fluoroscopy, or ultrasound. Imaging provides crucial information about the locations of the clinical target, anatomical obstacles, and the needle itself during the procedure. Accurate guidance of the needle tip is often crucial to the success of such image-guided procedures. For example, inaccurate needle tip placement may result in misdiagnosis during biopsy and unsuccessful cancer treatment during brachytherapy 84].

Needle insertion is traditionally performed using rigid needles, but recent advancements in steerable needles have the potential to enable clinicians to more accurately reach clinical targets while simultaneously avoiding anatomical obstacles 21,25]. Unlike rigid needles that are restricted to approximately straight line paths from the needle entry location to the clinical target, flexible needles with an asymmetric, bevel tip naturally move along a curve when inserted into soft tissue 28,29 . A steerable needle's insertion trajectory can be adjusted during a procedure to improve the accuracy of reaching moving tar- 
gets, e.g., target perturbations of approximately $7.0 \mathrm{~mm}$ are common during clinical interventions in breast tissue 85,88 . The ability to control a steerable needle along curved trajectories also enables these needles to reach previously inaccessible targets while avoiding anatomical obstacles, including impenetrable structures such as bones and sensitive structures such as blood vessels or nerves.

We introduce a needle steering system capable of autonomously and accurately guiding a steerable needle using two-dimensional (2D) ultrasound imaging to a moving target while avoiding a moving obstacle in three-dimensional (3D) anatomy. Ultrasound imaging is an ideal imaging modality to use during needle insertion procedures because of its low cost compared to CT and MR, and because it does not rely on ionizing radiation, which can be harmful to the patient when used in large doses during continuous CT or fluoroscopy imaging 89. However, ultrasound is challenging to use for needle tracking because of its low resolution and high noise. We use a novel technique to track and steer flexible needles in 3D using 2D ultrasound images. The $2 \mathrm{D}$ ultrasound transducer is placed at the tissue surface perpendicular to the direction of needle insertion (Fig. 2.1). During needle insertion, the method automatically repositions the transducer such that the needle tip is in the imaging plane. Our method also processes the images to estimate the needle tip pose, enabling online tracking of the needle tip in 3D anatomy.

In this study, we integrate ultrasound tracking into a complete system capable of automatic needle steering in non-static environments in which obstacles and targets may move. The system includes a motion planner that, given the pose of the needle estimated from ultrasound, computes a feasible trajectory that optimizes a clinical criterion and steers the needle around obstacles to a target in a $3 \mathrm{D}$ environment. The system is capable of considering and correcting for obstacle and target motions, and perturbations in the trajectory of the needle due to real-world uncertainties. This is possible because of the motion planner which is sufficiently fast such that it can be executed in a closed-loop manner. Closed-loop planning enables the needle trajectory to be continuously updated as online feedback is obtained from ultrasound tracking. Our system provides a novel approach to controlling steerable needles in $3 \mathrm{D}$ under $2 \mathrm{D}$ ultrasound image guidance.

To the best of our knowledge, our results are the first to experimentally demonstrate a needle steering system that, (1) integrates 3D steerable needle tracking using 2D ultrasound images and 3D motion planning, and (2) successfully guides the needle to a moving target while avoiding a moving obstacle. Our system is capable of accurately placing the needle tip at the desired target location (e.g., lesion), which is essential for successful diagnosis or therapy in many clinical applications. Potential applications that could benefit from this kind of system include breast biopsy and prostate brachytherapy. 
Chapter 2. Needle path planning and steering in a three-dimensional non-static environment using two-dimensional ultrasound images

\subsection{Related Work}

Our work builds on the following two main areas of research for improving the accuracy of needle guidance in soft tissues: needle tracking and needle steering.

A key aspect of improving needle targeting accuracy is accurately tracking the needle tip during a clinical procedure, which is complicated by the limitations of medical imaging modalities. The spatial resolution of $3 \mathrm{D}$ ultrasound images is limited and the refresh rate of a 3D image is low 39. The use of $\mathrm{x}-$ ray-based imaging such as CT or fluoroscopy exposes the patient to high doses of ionizing radiation 89,90 . MR imaging suffers from low refresh rate and incompatibility with ferromagnetic materials 91. Electromagnetic position tracking sensors 21,92 can be used for 3D needle tracking, but their accuracy is sensitive to ferrous materials in the range of measurement. Further, studies by Hong et al. and Neubach and Shoham provided ultrasound-based tracking methods for needles, but motion is limited to the imaging plane 32,41 . A study by Neshat and Patel used 2D ultrasound images to construct a volume, but the volume size remains limited by the available acquisition time in real-time applications [40]. Recently, Vrooijink et al. presented a method to online track flexible needles in 3D using 2D ultrasound images [45]. Our study expands on this technique to track and steer the needle using 2D ultrasound images in the presence of both obstacle and target motion.

Needle steering techniques and devices have been introduced that enable clinicians to improve targeting accuracy by adjusting the needle path within tissue. Such needle steering techniques and devices include bevel-tip flexible needles [28], symmetric-tip needles that can be steered by applying forces at the base [22, 38, curved stylet tips [23, programmable bevel-tip needles [26], and pre-bent concentric tubes [27,31]. We focus on bevel-tip flexible needles. Significant advancements have been made in modeling bevel-tip steerable needles 25. Webster et al. developed and experimentally validated a kinematicbased model based on a unicycle 28]. Minhas et al. showed that the curvature of the needle path can be controlled through duty cycled spinning of the needle during insertion 24]. Misra et al. and Majewicz et al. modeled the characteristics and mechanics of steerable needles in soft-tissue phantoms and biological tissue, respectively 29,43 .

There is extensive research on motion planning and control of steerable needles in a plane (2D) [46,51,53. DiMaio and Salcudean presented a path planning algorithm that relates the needle motion at the base (outside the soft-tissue phantom) to the tip motion inside the soft-tissue phantom $[22$. Motion planners have been developed for needle steering in 3D environments with obstacles. Duindam et al. proposed a fast planner based on inverse kinematics, but which offers no completeness guarantee [48. Park et al. proposed a path-of-probability algorithm that considers uncertainty in needle motion using diffusion-based error propagation, but the planner is not guaranteed to be complete when obstacles are present [49]. Several studies presented 3D path 
planning approaches based on Rapidly-exploring Random Trees (RRTs) [47 50]. These studies demonstrated results in simulations and have not been validated experimentally in 3D under closed-loop ultrasound image guidance.

Needle steering algorithms to control the needle to follow a planned path have been developed. Glozman and Shoham created an image-guided closedloop control algorithm for steering flexible needles using fluoroscopic images for feedback of the needle position during insertion [38]. Neubach and Shoham and Abayazid et al. used ultrasound images for 2D steering [41,55]. A recent study by Bernardes et al. demonstrated a robot-assisted approach for automatic steering of flexible bevel-tipped needles 53]. The 2D needle steering method operates in closed-loop using camera images for feedback, while intraoperative trajectory replanning is used to deal with obstacles and dynamic workspaces. Another study by Abayazid et al. uses Fiber Bragg Grating sensors for 3D closed-loop needle steering without path-planning [56]. Hauser et al. developed a 3D feedback controller that steers the needle along a helical path, but the results were only validated in simulations [54]. Van den Berg et al. proposed a framework for planning and Linear-quadratic-Gaussian (LQG)-based feedback control of a steerable needle under motion and sensing uncertainty, which was extended by Patil et al. for deformable workspaces [93, 94]. Despite these advances, prior LQG-based methods may fail due to control saturation, which is a practical concern for needle steering. Furthermore, these prior LQG-based methods cannot respond in real-time to significant perturbations that are not considered a priori.

The majority of the mentioned studies demonstrated needle steering in 2D. Even fewer studies investigated 3D steering that are also experimentally evaluated. Our study is the first to describe an ultrasound-guided 3D needle steering system capable of avoiding obsacles and reaching targets in non-static environments. This novel system effectively integrates the online ultrasound-based 3D tracking method described by Vrooijink et al. with the motion planner presented by Patil et al., which we extend in this study to execute in a closed-loop manner under ultrasound guidance 45,94$]$. We use duty cycled spinning to achieve variable needle curvature in order to steer the flexible needle along the trajectory computed by the motion planner. The integrated system is capable of steering needles in non-static environments while compensating for uncertainties such as perturbations in needle motion and a priori unknown motions in obstacle and target locations. We experimentally evaluated the targeting accuracy of the system in static and non-static scenarios using a soft-tissue phantom.

\subsection{Methods}

In this section, we present methods to enable robot-assisted tracking and steering of flexible bevel-tipped needles. We summarize the needle tip tracking 
Chapter 2. Needle path planning and steering in a three-dimensional non-static environment using two-dimensional ultrasound images

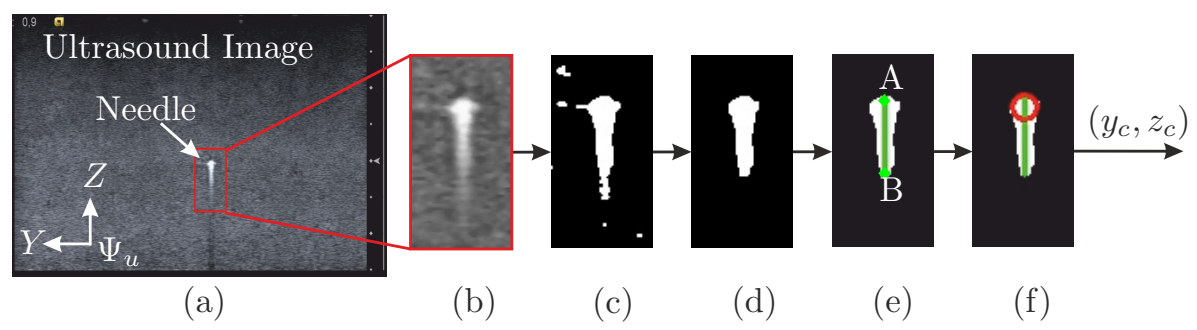

Figure 2.2: The ultrasound image processing steps performed to determine the needle centroid position $\left(y_{c}, z_{c}\right)$. (a) The ultrasound image shows a radial cross-sectional view of the needle affected by the comet tail artifact (CTA). A cropped portion is used for image processing. (b) A median filter is applied to reduce speckle in the ultrasound image. (c) Thresholding is used to obtain a binary image of the needle. (d) Erosion and subsequently dilation are applied to remove the remaining speckle in the ultrasound image. (e) A feature extraction algorithm (Hough Transform) is used to find a vertical line segment denoted by $\overline{A B}$, which describes the needle with CTA. (f) The needle centroid position $\left(y_{c}, z_{c}\right)$ is evaluated from $A$ in the direction of $B$ at a distance equal to the needle radius, and displayed as the center of the red circle.

method which uses 2D ultrasound images to estimate the pose of the needle tip during insertion. The tip pose is used in motion planning and steering, which allows the needle to be steered in a non-static environment with obstacle and target motion.

\subsubsection{Ultrasound Image Processing}

Our system processes ultrasound images to estimate the needle tip pose, which is used for needle steering. The 2D ultrasound transducer is placed perpendicular to the needle insertion axis, as shown in Fig. 2.1. The resulting 2D ultrasound image provides a radial cross-sectional view of the needle, which has ideally a circular shape. However, the radial cross-sectional view of the needle is deformed by an artifact known as reverberation (Fig. 2.2(a)). The artifact occurs when sound waves reflect repeatedly between materials with different acoustic impedances 95 . The acoustic impedance difference between needle and soft tissue causes sound waves to bounce multiple times inside the needle before exiting. If the angle of the reflected sound waves are almost perpendicular to the receivers in the transducer, the reflected sound waves will produce an artifact. The artifact, often referred to as a comet tail artifact (CTA), is visible in ultrasound images and has a tail-shaped structure of equally spaced echoes along the sound wave [96]. The length of the tail-shaped structure depends on the amount of bouncing echoes that are received by the transducer.

We developed an image processing method to locate the needle centroid from the radial cross-sectional view of the needle which is affected by the CTA. Our method consists of a series of image processing techniques used to determine the needle centroid independent of the influences of the CTA. In this 
study, we assume that ultrasound images are properly de-wrapped and scaled. We first process the ultrasound images to enhance the needle using a series of basic image processing techniques, including median filtering, thresholding, and erosion and dilation in Fig. 2.2(b), (c) and (d), respectively. The enhanced image of the needle is used to determine the needle centroid. We apply to the enhanced image a feature extraction algorithm based on the Hough transform to compute a set of vertical line segments which describe the needle cross section and CTA. The length of each line segment must be equal or greater than the needle diameter. The algorithm then computes the mean line segment $(\overline{A B})$ of the set of vertical line segments (Fig. 2.2(e)). The line segment $(\overline{A B})$ describes the location and height of needle cross section and CTA under the assumption that the tail-shaped structure of the CTA is symmetric along the sound wave. Variations in the size of the tail-shaped structure are dependent on the amount of echoes that return to the transducer and affect the mean line segment at $B$. Point $A$ of mean line segment $(\overline{A B})$ is not affected by the CTA, and represents a point on the surface of the needle which is used to determine the needle centroid location. We estimate the needle centroid $\left(y_{c}, z_{c}\right)$ as the point on the line segment between $A$ and $B$ a distance equal to the radius of the needle from $A$ (Fig. 2.2(f)). By positioning the transducer at the needle tip during insertion, we can estimate the needle tip position (centroid $\left(y_{c}, z_{c}\right)$ ), which can be used to estimate the needle tip pose as described below.

\subsubsection{Needle Tip Pose Estimation}

The coordinate frames required to determine the needle tip pose during insertion are shown in Fig. 2.3. The needle is inserted in the soft-tissue phantom along the $x$-axis (frame $\left(\Psi_{0}\right)$ ) with insertion velocity $\left(\mathbf{v}_{i}\right)$ using a needle insertion device (NID). The NID also enables needle rotation about the $x$-axis (frame $\left(\Psi_{0}\right)$ ), which allows the needle to bend in a controlled direction. In order to determine the needle tip pose as it moves through the soft-tissue phantom, the needle tip position,

$$
\mathbf{p}_{t}^{0}=\left[\begin{array}{lll}
p_{x} & p_{y} & p_{z}
\end{array}\right]^{T}
$$

with respect to the fixed reference frame $\left(\Psi_{0}\right)$ is evaluated. The needle centroid $\left(y_{c}, z_{c}\right)$, describes the estimated tip frame $\left(\Psi_{\hat{t}}\right)$ in the ultrasound image frame $\left(\Psi_{u}\right)$. The frames $\left(\Psi_{u}\right.$ and $\left.\Psi_{p}\right)$ are considered coincident for computational simplicity. Frame $\left(\Psi_{p}\right)$ is attached to the positioning device end-effector, and is used to describe the transducer position with respect to fixed reference frame $\left(\Psi_{0}\right)$. Thus, by using coordinate transformations, the estimated needle tip position $\left(\mathbf{p}_{\hat{t}}^{0}\right)$ can be expressed in the fixed reference frame $\left(\Psi_{0}\right)$.

In order to estimate the needle tip position $\left(\mathbf{p}_{\hat{t}}^{0}\right)$, the ultrasound image plane must be located at the tip. Therefore, the transducer needs to be repositioned along the insertion axis ( $x$-axis of frame $\left(\Psi_{0}\right)$ ) according to the needle tip motion. It is assumed that the needle does not buckle during insertion. Hence, 
Chapter 2. Needle path planning and steering in a three-dimensional non-static environment using two-dimensional ultrasound images

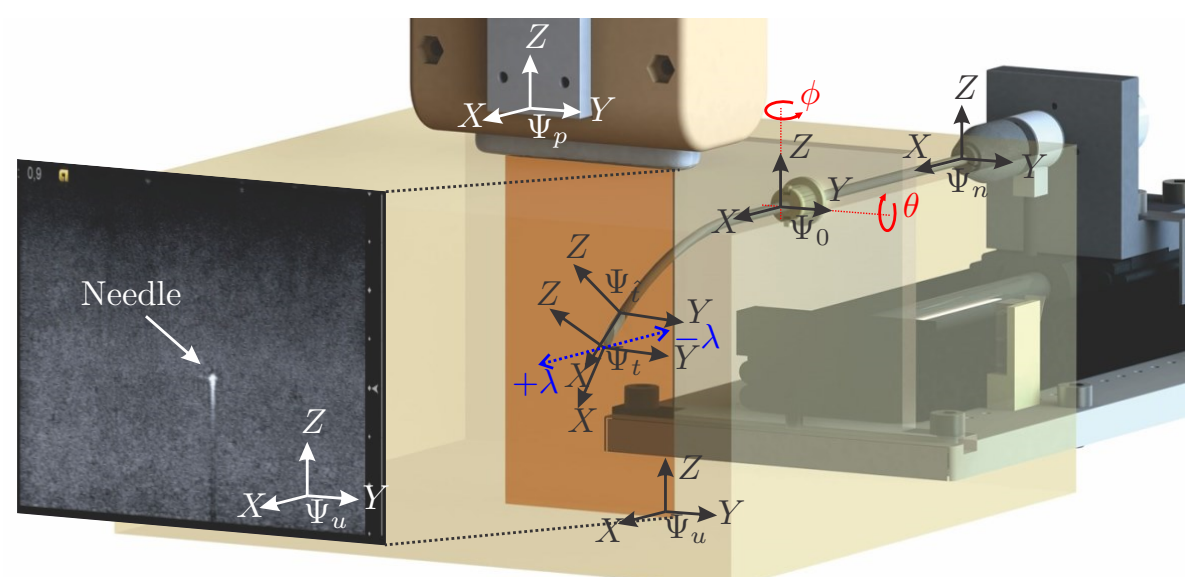

Figure 2.3: The coordinate frames used to estimate the needle tip pose: Frame $\left(\Psi_{0}\right)$ is used as fixed reference frame located at the needle entry point. Frame $\left(\Psi_{n}\right)$ is attached to the needle insertion device end-effector, while frame $\left(\Psi_{p}\right)$ is located at the end-effector of the transducer positioning device. Frame $\left(\Psi_{u}\right)$ is fixed to the ultrasound image plane. Frame $\left(\Psi_{t}\right)$ is located at the needle tip, while frame $\left(\Psi_{\hat{t}}\right)$ is fixed at the estimated needle tip location. The ultrasound transducer aberration along the needle insertion axis $(x$-axis of frame $\left.\left(\Psi_{0}\right)\right)$ is denoted by $\pm \lambda$. Furthermore, the orientation of the needle tip about the $y$ and $z$-axes of frame $\left(\Psi_{0}\right)$ is indicated by $\theta$ and $\phi$, respectively.

the needle tip velocity $\left(\left\|\dot{\mathbf{p}}_{t}^{0}\right\|\right)$ equals to the insertion velocity $\left(\left\|\mathbf{v}_{i}\right\|\right)$ at the base,

$$
\left\|\mathbf{v}_{i}\right\|=\sqrt{\dot{p}_{x}^{2}+\dot{p}_{y}^{2}+\dot{p}_{z}^{2}}
$$

This relation can be used to estimate the required transducer motion along the $x$-axis (frame $\left(\Psi_{0}\right)$ ) to compensate for the needle tip motion. Thus, by rewriting 2.2 , the required transducer motion is given by

$$
\dot{\hat{p}}_{x}=\sqrt{\left\|\mathbf{v}_{i}\right\|^{2}-\dot{\hat{p}}_{y}{ }^{2}-\dot{\hat{p}}_{z}{ }^{2}}
$$

where the insertion velocity is corrected by estimated tip velocities $\left(\dot{\hat{p}}_{y}\right.$ and $\left.\dot{\hat{p}}_{z}\right)$ which are the derivatives of needle tip positions $\left(\hat{p}_{y}\right.$ and $\left.\hat{p}_{z}\right)$, respectively.

In order to determine the needle tip pose, orientations about the $x$ - $(\psi)$ , $y$ - $(\theta)$ - and $z$ - $(\phi)$-axes are required. The NID controls the needle tip orientation $(\psi)$ about its $x$-axis. If we assume no torsional flexibility about the needle shaft, the bevel tip orientation of the needle (about the $x$-axis of frame $\left(\Psi_{t}\right)$ ) can be determined from the NID (frame $\left(\Psi_{n}\right)$ ). The orientation of the needle tip about the $y$ - $(\theta)$ - and $z$ - $(\phi)$-axes of frame $\left(\Psi_{0}\right)$ as depicted in Fig. 2.3 are computed by

$$
\theta=\operatorname{atan} 2\left(\Delta \hat{p}_{z}, \Delta \hat{p}_{x}\right) \text { and } \phi=\operatorname{atan} 2\left(\Delta \hat{p}_{y}, \Delta \hat{p}_{x}\right)
$$




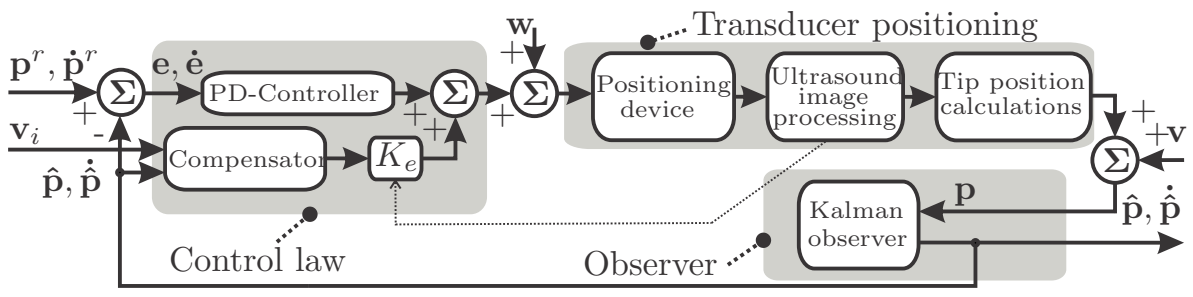

Figure 2.4: An overview of the controller architecture to control the transducer motion in order to enable online three-dimensional needle tip tracking. The transducer motion along the $x$-axis (frame $\left(\Psi_{0}\right)$ ) is evaluated by the compensator using the needle insertion velocity $\left(\mathbf{v}_{i}\right)$ according to 2.3 . In order to provide closed-loop control, gain scheduling of $K_{e}$ according to 2.8 is applied. In-plane motion $\left(y\right.$-axis of frame $\left.\left(\Psi_{0}\right)\right)$ of the needle tip is compensated for by a proportional-derivative-(PD)-controller (proportional gain $\left(K_{p}=\right.$ $0.4)$ and derivative gain $\left(K_{d}=0.1\right)$ ). The needle tip motion in the $z$-axis (frame $\left.\left(\Psi_{0}\right)\right)$ is not compensated for. The $z$-axis (frame $\left(\Psi_{0}\right)$ ) is used to position the transducer on the surface of the soft-tissue phantom. The transducer motion is enabled by a Cartesian positioning device to provide the needle tip position $(\mathbf{p})$. The needle tip velocity $(\dot{\mathbf{p}})$ is obtained by taking the time derivative of $\mathbf{p}$. The tracker reference position and velocity signals are denoted $\mathbf{p}^{r}$ and $\dot{\mathbf{p}}^{r}$, while the tracker position and velocity errors are denoted $\mathbf{e}$ and $\dot{\mathbf{e}}$, respectively. The influence of process $(\mathbf{w})$ and measurement $(\mathbf{v})$ noise on the states ( $\mathbf{p}$ and $\dot{\mathbf{p}})$ are minimized by a Kalman observer, which also predicts the subsequent state. The estimated needle tip position and velocity are denoted by $\hat{\mathbf{p}}$ and $\dot{\hat{\mathbf{p}}}$, respectively.

respectively, where $\Delta \hat{p}_{x}, \Delta \hat{p}_{y}$ and $\Delta \hat{p}_{z}$ represents small needle tip displacements along the $x-, y$ - and $z$-axes of frame $\left(\Psi_{0}\right)$, respectively. The rotation matrix $\left(\mathbf{R}_{\hat{t}}^{0}\right)$ is computed using the tip orientations $(\psi, \theta$ and $\phi)$. The tip pose is known, since position $\left(\mathbf{p}_{\hat{t}}^{0}\right)$ and orientation $\left(\mathbf{R}_{\hat{t}}^{0}\right)$ are known. Thus, the homogeneous transformation $\left(\mathbf{H}_{\hat{t}}^{0}\right)$ is estimated by

$$
\mathbf{H}_{\hat{t}}^{0}=\left[\begin{array}{cc}
\mathbf{R}_{\hat{t}}^{0} & \mathbf{p}_{\hat{t}}^{0} \\
\mathbf{0}_{3}^{T} & 1
\end{array}\right],
$$

which describes the estimated needle tip frame $\left(\Psi_{\hat{t}}\right)$ with respect to the reference frame $\left(\Psi_{0}\right)$. In order to estimate the needle tip pose during insertion, we implemented a controller to accurately position the ultrasound transducer at the needle tip.

\subsubsection{Ultrasound Image-Guided Controller}

The ultrasound transducer is positioned at the needle tip using the controller architecture presented in Fig. 2.4. The needle tip position is denoted by $\mathbf{p}$, and its corresponding time derivative representing the tip velocity is given by $\dot{\mathbf{p}}$. Unless otherwise stated, the variables used in this subsection are expressed in the fixed reference frame $\left(\Psi_{0}\right)$, which is not included for notational simplicity. The transducer moves in the needle insertion direction $\left(x\right.$-axis of frame $\left(\Psi_{0}\right)$ ) using a compensator according to the estimated needle tip velocity $\left(\dot{\hat{p}}_{x}\right) 2.3$. 
Chapter 2. Needle path planning and steering in a three-dimensional non-static environment using two-dimensional ultrasound images

Estimation errors in the needle tip velocity $\left(\dot{\hat{p}}_{x}\right)$ caused by sideways cutting or needle deformation results in a positioning error between transducer and needle tip along the $x$-axis (frame $\left(\Psi_{0}\right)$ ), which is considered to be the transducer aberration denoted by $\lambda$ (Fig. 2.3). The aberration is given by

$$
\lambda=\left|p_{x}-\hat{p}_{x}\right|
$$

where $p_{x}$ represents the needle tip position and $\hat{p}_{x}$ the estimated tip position by the controller. The transducer aberration $(\lambda)$ introduces an error in the estimated needle tip pose $\left(\mathbf{H}_{\hat{t}}^{0}\right)$,

$$
\mathbf{H}_{\hat{t}}^{t}=\mathbf{H}_{0}^{t} \mathbf{H}_{\hat{t}}^{0}
$$

where $\mathbf{H}_{\hat{t}}^{t} \in \mathbb{R}^{4 \times 4}$ represents the pose error between frames $\left(\Psi_{t}\right.$ and $\left.\Psi_{\hat{t}}\right)$, which ideally equals the identity matrix.

Closed-loop control is applied to reduce the transducer aberration $(\lambda)$ that introduces the needle tip pose error $\left(\mathbf{H}_{\hat{t}}^{t}\right)$. This is achieved by adding a gain $\left(K_{e}\right)$ to the estimated needle tip velocity $\left(\dot{\hat{p}}_{x}\right)$. Thus, by scheduling of $K_{e}$, the transducer velocity can be increased $\left(K_{e}>1\right.$ to move faster than the needle) or decreased $\left(K_{e}<1\right.$ to move slower than the needle) when the needle is in- or out-of-plane, respectively. The velocity gain is scheduled according to

$$
K_{e}=\left\{\begin{array}{ll}
1.05 & \text { if needle is in-plane } \\
0.5 & \text { if needle is out-of-plane }
\end{array},\right.
$$

where closed-loop control is achieved by estimating $K_{e}$ empirically. The imposed gain scheduling controller forces the transducer to move towards the needle tip, and thus, minimizes $\lambda$ and therefore minimizes the needle tip pose error $\left(\mathbf{H}_{\hat{t}}^{t}\right)$.

A standard proportional-derivative (PD) controller is used to control the transducer motion along the $y$-axis (frame $\left(\Psi_{0}\right)$ ), which allows the needle tip to move beyond the transducer image width $(5.5 \mathrm{~cm})$. The transducer motion along the $z$-axis (frame $\left(\Psi_{0}\right)$ ) is used to maintain contact between the transducer and surface of the soft-tissue phantom to provide clear ultrasound images. In order to minimize the influence of process and measurement noise on the states ( $\mathbf{p}$ and $\dot{\mathbf{p}}$ ), a Kalman observer is included 97 . The discrete state-space representation is given by $\mathbf{x}(k+1)=\mathbf{A x}(k)$ and $\mathbf{y}(k)=\mathbf{C x}(k)$, where $k$ is the discrete-time index, $\mathbf{x}=\left[\begin{array}{ll}\mathbf{p} & \dot{\mathbf{p}}\end{array}\right]^{T}, \mathbf{C}=\left[\begin{array}{ll}\mathbf{1} & \mathbf{0}\end{array}\right], \mathbf{1}$ and $\mathbf{0}$ are $1 \times 3$ row vectors filled with ones and zeros, respectively, and $\mathbf{A}=\left[\begin{array}{ll}\mathbf{a}_{\mathbf{0}} & \mathbf{a}_{\mathbf{1}}\end{array}\right]$, where $\mathbf{a}_{\mathbf{0}}=\left[\begin{array}{ll}\mathbf{I} & \mathbf{0}_{3}\end{array}\right]^{T}, \mathbf{a}_{\mathbf{1}}=\left[\begin{array}{ll}\Delta t \mathbf{I} & \mathbf{I}\end{array}\right]^{T}, \mathbf{I}$ is a $3 \times 3$ identity matrix, $\mathbf{0}_{3}$ is a $3 \times 3$ matrix filled with zeros, and $\Delta t=0.04 \mathrm{sec}$ denotes the sampling time of the Kalman observer. The system and measurement covariances are given by $\mathbf{Q}=\left[\begin{array}{ll}\mathbf{q}_{0} & \mathbf{q}_{1}\end{array}\right]$, where $\mathbf{q}_{0}=\left[\begin{array}{ll}\frac{\Delta t^{4}}{4} \mathbf{I} & \frac{\Delta t^{3}}{2} \mathbf{I}\end{array}\right]^{T}$ and $\mathbf{q}_{1}=\left[\begin{array}{cc}\frac{\Delta t^{3}}{2} \mathbf{I} & \Delta t^{2} \mathbf{I}\end{array}\right]^{T}$ and $R=0.1$, respectively. Note, that the covariance of the system noise is obtained 


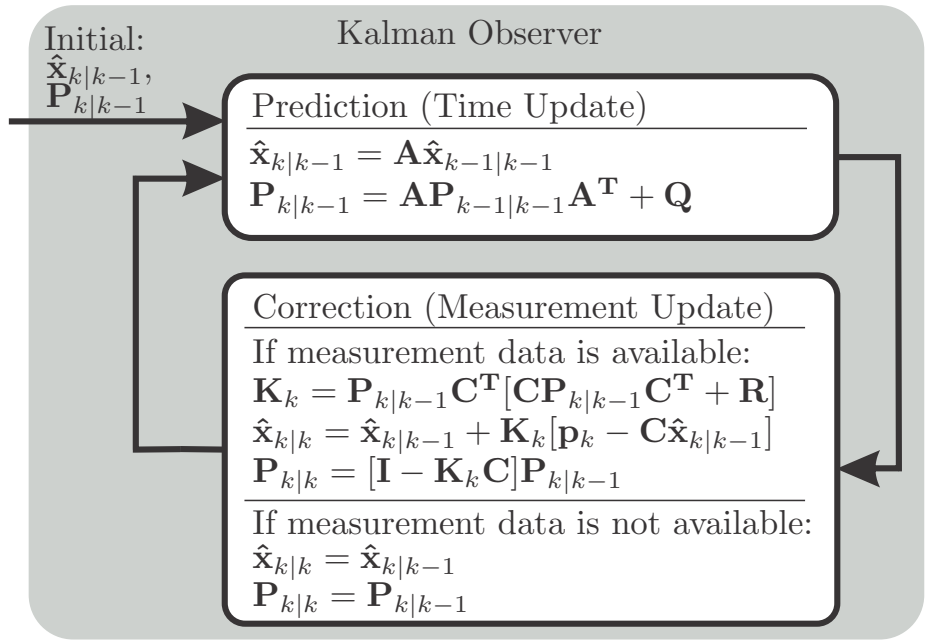

Figure 2.5: A schematic representation of the Kalman observer. During the time update, predictions of the state $\left(\hat{\mathbf{x}}_{k \mid k-1}\right)$ and the error covariance $\left(\mathbf{P}_{k \mid k-1}\right)$ are performed. Subsequently, if the needle is detected in the ultrasound image, a measurement update is performed with Kalman gain $\left(\mathbf{K}_{k}\right)$ and needle tip position measurement $\left(\mathbf{p}_{k}\right)$.

by $\mathbf{Q}=\mathbf{G G}^{T} \sigma_{a}^{2}$, where $\mathbf{G}=\left[\begin{array}{ll}\frac{\Delta t^{2}}{2} \mathbf{I} & \Delta t \mathbf{I}\end{array}\right]^{T}$ and it is assumed that forces acting on the needle cause a constant needle tip acceleration, which is normally distributed according to $\mathcal{N}\left(0, \sigma_{a}\right)$ with $\sigma_{a}=1$. A schematic representation of the Kalman observer is described in Fig. 2.5. Another important role of the Kalman observer is to provide state estimation when the transducer moves ahead of the needle (which results in loss of needle visibility). This allows the compensator described in Fig. 2.4 to reposition the ultrasound transducer according to the estimated needle tip velocity $\left(\dot{\hat{p}}_{x}\right) \sqrt{2.3}$. The uncertainty of the projected states increases over time without measurement updates. Hence, it is essential to minimize the duration of measurement absence. Upon return of measurement data, the Kalman gain $\left(\mathbf{K}_{k}\right)$ is adapted according to the increased uncertainty of the projected states, ensuring a decrease in estimation error.

The images of the ultrasound machine are transferred to a computer and processed at 25 frames-per-second. The controller architecture with the Kalman observer, depicted in Fig. 2.4. operates at 25 [Hz]. This facilitates repositioning of the ultrasound transducer in order to track the needle during insertions with velocities $1-5[\mathrm{~mm} / \mathrm{s}]$. Higher insertion velocities could be considered, but this is associated with an increase of the aberration in transducer position. Tracking according to the proposed method was validated with maximum mean errors of $0.64 \pm 0.11 \mathrm{~mm}, 0.25 \pm 0.06 \mathrm{~mm}$ and $0.27 \pm 0.06 \mathrm{~mm}$ along the $x-, y$ and $z$-axes, respectively. The error in tip orientations about the $y$ - $(\theta)$ - and $z$ - 
Chapter 2. Needle path planning and steering in a three-dimensional non-static environment using two-dimensional ultrasound images

$(\phi)$-axes are $2.68^{\circ} \pm 1.22$ and $2.83^{\circ} \pm 1.36$, respectively. We experimentally evaluated transducer position aberration using insertion velocities $1-5[\mathrm{~mm} / \mathrm{s}]$, which resulted in a mean aberration of $0.24-0.64[\mathrm{~mm}]$. We refer the reader to Vrooijink et al. for details regarding the experiments performed to evaluate needle tip tracking [45]. The proposed method evaluates the needle tip pose $\left(\mathbf{H}_{\hat{t}}^{0}\right)$ at $25[\mathrm{~Hz}]$ during insertion. The estimated needle tip pose is used in a separate motion planning loop in order to steer the needle to a desired target while avoiding obstacles as described below.

\subsubsection{Motion Planning}

We use a fast motion planner to automatically compute motions that steer the needle's tip to a moving target while avoiding a moving obstacle in a 3D environment. Given preoperative medical images, we assume the user specifies the clinical target as well as obstacles, including sensitive structures such as glands or blood vessels and impenetrable structures such as bones. The objective of the motion planner is to quickly compute a sequence of feasible motions that steer the needle's tip from its current pose to the target while avoiding obstacles. Our motion planner, described below, is fast enough to execute in a closed-loop manner to correct for perturbations in needle motion, obstacle location, and target location as they occur.

At the core of our closed-loop motion planning approach is a sampling-based rapidly exploring random tree (RRT) planner 98 that is customized for needle steering [50], as outlined in Alg. 1 .

Prior work on motion planning for steerable needles in 3D assumes a constant curvature kinematic model, which severely restricts the range of motion of the needle tip 47, 48. This makes it difficult for planners to compute a feasible motion plan in 3D environments with obstacles. In contrast, our planner assumes a variable curvature kinematic model that allows us to compute trajectories composed of circular arcs of bounded curvature and uses duty cycled spinning during insertion to adjust the needle's net curvature [24], as described in the next subsection. The planner also makes use of reachability-guided sampling for efficient expansion of the rapidly-exploring search tree to significantly improve planner performance 99]. These customizations help us to achieve orders-of-magnitude reduction in computation time compared to prior sampling-based planners 47]. In this work, we extend the motion planner to operate in a closed-loop manner with ultrasound imaging feedback.

The input to the planner is the estimated needle tip pose $\left(\mathbf{H}_{\hat{t}}^{0}\right)$, a target region $\left(\mathcal{P}_{\text {target }}\right)$, and the computation time $(\Delta)$ allotted for planning. The planner incrementally builds a tree $(\mathcal{T})$ over the state space, while satisfying nonholonomic motion constraints of the needle and avoiding obstacles in the environment. To expand the tree $(\mathcal{T})$, a random point $\left(\mathbf{p}_{\text {rand }} \in \mathbb{R}^{3}\right.$ ) is sampled from the workspace. The algorithm then identifies a node $\left(\mathbf{H}_{\text {near }}\right)$ in the tree, that is closest (i.e., minimizes distance) to the sample $\left(p_{\text {rand }}\right)$. For fast perfor- 


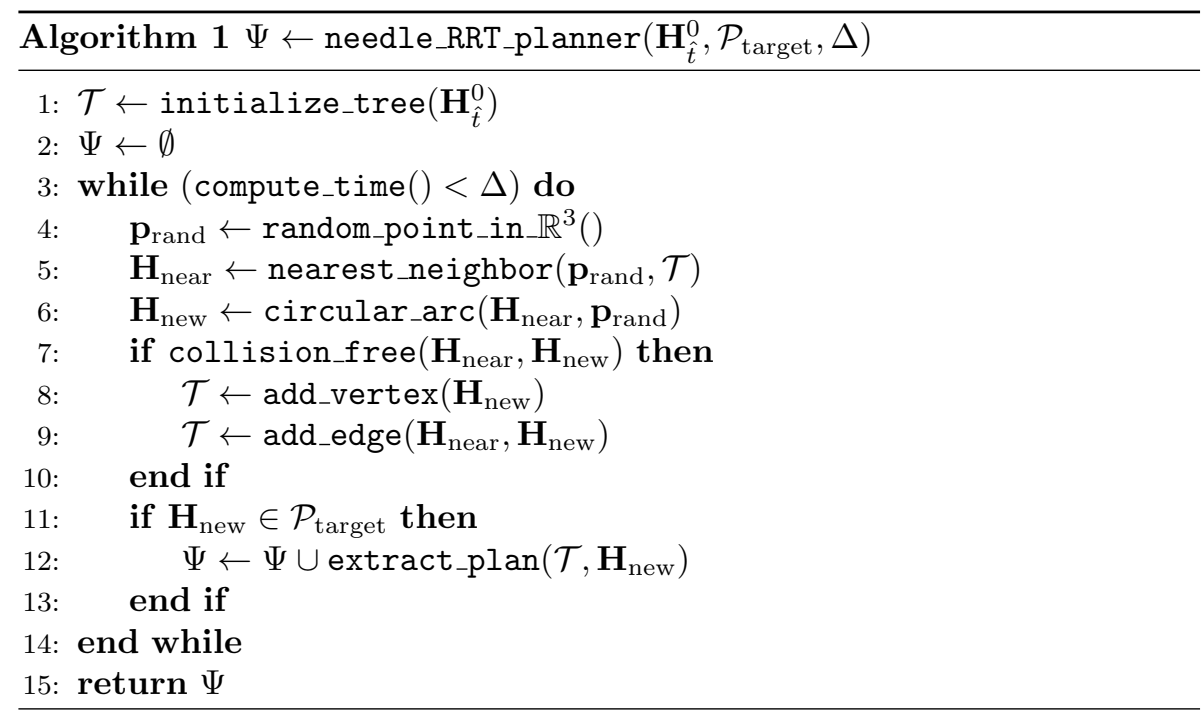

mance, we use a distance metric customized for steerable needles that accounts for the needle's nonholonomic constraint 50. Since the needle has a natural maximum curvature $\left(\kappa_{0}\right)$, not all sampled points will be reachable from a given state because of the nonholonomic constraints of the needle. The reachable set from a state $\mathbf{H}_{\text {near }}=\left[\begin{array}{cc}R_{\text {near }} & \mathbf{p}_{\text {near }} \\ \mathbf{0}_{3}^{T} & 1\end{array}\right]$ consists of all points that can be connected to $\mathbf{p}_{\text {near }}$ by a circular arc that has a radius $r \geq 1 / \kappa_{0}$ and is tangent to the

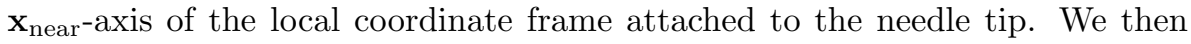
define the distance metric as the length of such a circular arc connecting $\mathbf{p}_{\text {rand }}$ and $\mathbf{H}_{\text {near }}$ if $\mathbf{p}_{\text {rand }}$ is in the reachable set of $\mathbf{H}_{\text {near }}$, and infinity otherwise. This strategy restricts the search domain to only those nodes that are within the reachable set of the nearest node $\left(\mathbf{H}_{\text {near }}\right)$, thus increasing the likelihood of coverage of the state space 99].

The sampled point ( $\mathbf{p}_{\text {rand }}$ ) can then be connected to $\mathbf{H}_{\text {near }}$ directly using a circular arc parameterized by $[l, \phi, r]^{T}$, where $l$ is the arc length, $\phi$ is the change in orientation of the needle tip coordinate frame $\left(\mathbf{H}_{\text {near }}\right)$ around the $\mathbf{x}_{\text {near-axis, }}$ and $r$ is the arc radius. We limit the length $(l)$ of the arcs that are added. Let $\mathbf{H}_{\text {new }}$ be the state reached by traversing along the circular arc starting from $\mathbf{H}_{\text {near }}$ and traveling a maximum distance of $l \leq l_{\max }$. We add $\mathbf{H}_{\text {new }}$ and the edge connecting $\mathbf{H}_{\text {near }}$ and $\mathbf{H}_{\text {new }}$ to the tree $(\mathcal{T})$ if the circular arc connecting the two states is collision free. When the position $\mathbf{p}_{\text {new }}$ of a newly added state $\left(\mathbf{H}_{\text {new }}\right)$ is found to lie in the target region $\left(\mathcal{P}_{\text {goal }}\right)$, we extract a planned path by traversing the tree $(\mathcal{T})$ backwards from $\mathbf{H}_{\text {new }}$ to the root. We refer the reader to Patil et al. for further details on our RRT-based planning approach [50].

The output of the planner is a set of plans $(\Psi)$ that can be computed in the 
Chapter 2. Needle path planning and steering in a three-dimensional non-static environment using two-dimensional ultrasound images

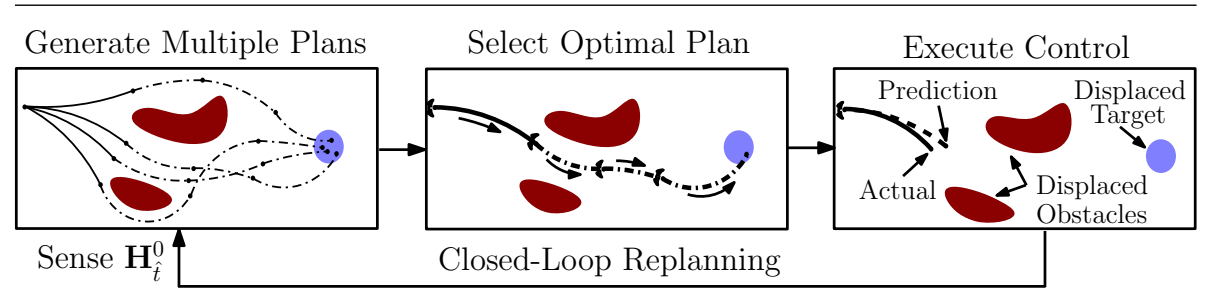

Figure 2.6: We use closed-loop motion planning to steer the needle to a target. Given a needle tip pose and the locations of a target region and obstacles, our fast, randomized motion planner computes in the available time many feasible motion plans (left). The method selects the best plan based on clinically motivated optimization criteria such as minimizing path length or maximizing clearance from obstacles (middle). The needle insertion device then executes the first control output of the plan (right). The planner is periodically executed every $\Delta$ seconds, closing the loop. At the beginning of each period, the ultrasound system returns an estimate of the needle tip pose, and the motion planner executes for the next period while the needle insertion device executes the previously computed control output. Closed-loop motion planning enables the system to automatically steer the needle to targets in $3 \mathrm{D}$ environments while avoiding obstacles and correcting for perturbations in needle motion and obstacle and target locations as they occur.

time $(\Delta)$ allotted for planning. We then select the best plan based on clinically motivated criteria such as minimizing path length or maximizing clearance from obstacles. In each period, multiple feasible motion plans are computed and a high quality plan is selected based on clinically motivated criteria. In our experiments, we minimize the length of the path (to minimize tissue cut) when no obstacles are present, and we maximize clearance from obstacles (to maximize safety) when obstacles are present.

The system executes the motion planner repeatedly during the procedure until the target is reached (see Fig. 2.6). After the user specifies the environment, the planner first computes an initial plan. The system then enters a closed-loop in which the planner is periodically re-executed every $\Delta$ seconds. The closed-loop motion planner as depicted in Fig. 2.6 is given a fixed planning time $(\Delta)$ of 0.6 sec. At the beginning of each period of duration $\Delta$, the motion planner obtains the actual needle tip pose $\left(\mathbf{H}_{\hat{t}}^{0}\right)$ from the ultrasound tracking system. The NID then executes the first $\Delta$ time of the previously computed plan. Simultaneously, the motion planner computes an updated plan that will be ready for execution in $\Delta$ time. The new motion plan is computed from a prediction of the needle tip pose after $\Delta$ time, where the prediction is based on the prior plan. The planner also uses the current positions of the target and the obstacles at each re-planning period. At the end of each period, the control outputs, consisting of the needle insertion and rotational velocities for the next $\Delta$, are sent to the NID for execution, and the process is repeated till the needle reaches the target. 


\subsubsection{Duty Cycled Needle Steering}

When a flexible bevel-tipped needle is inserted into soft tissue, the bevel tip causes the needle to follow a circular path with a radius of curvature that is approximately constant 28]. However, the planner computes a sequence of variable curvature circular arcs that steers the needle from the specified needle tip pose to the target. We approximate any curvature $(\kappa)$ between 0 and the maximum natural curvature $\left(\kappa_{0}\right)$ by duty cycling the rotation (spinning) of the needle 24 . The variable curvature (duty cycling) is achieved by alternating between (I) insertion with rotations, in which the needle moves straight by rotating (spinning) at a constant velocity, and (II) insertion without rotation, in which the needle follows a path of constant curvature. Needle spinning must be a multiple of full rotations in order to preserve the same bevel tip orientation every cycle.

Duty cycling is implemented for needle steering by inserting the needle a fixed distance each cycle and spinning with a fixed rotational velocity $\left(\omega_{\text {spin }}\right)$. Let $\delta$ be the duration of each duty cycling interval, which is composed of a spin interval of duration $\left(\delta_{\text {spin }}\right)$ and an insertion interval of duration $\left(\delta_{\text {ins }}\right)$, as illustrated in Fig. 2.7(a). Let $\alpha(0 \leq \alpha \leq 1)$ be the proportion of the time spent in spin intervals, i.e., $\alpha=\delta_{\text {spin }} / \delta$, where $\delta=\delta_{\text {spin }}+\delta_{\text {ins }}$. The empirical relationship between $\kappa$ and $\alpha$ is expressed as,

$$
\alpha=h(\kappa), 0 \leq \kappa \leq \kappa_{0},
$$

where $h(\kappa)$ is dependent on the mechanical properties of the needle and soft tissue, and is determined by fitting a polynomial function to the empirical data gathered during characterization experiments as described below.

Given a circular arc of desired curvature $(\kappa)$, we use 2.9 to determine $\alpha$. Since the needle tip preserves the same bevel tip orientation at the end of each spin interval, the duration of the spin interval is given by $\delta_{\text {spin }}=\left(2 k \pi / \omega_{\text {spin }}\right), k \in$ $\mathbb{Z}$. We then compute the quantities $\delta=\left(\delta_{\text {spin }} / \alpha\right)$ and $\delta_{\text {ins }}=\left(\delta-\delta_{\text {spin }}\right)$. The low level control inputs (NID) for the insertion velocity $(v(t))$ and rotational velocity $(\omega(t))$ during a duty cycle interval are given by

$$
\begin{aligned}
& v(t)=v_{\text {ins }}, \quad 0 \leq t \leq \Delta / \delta \\
& \omega(t)=\left\{\begin{array}{rll}
\omega_{\text {spin }} & \text { if } \quad j \delta<t \leq j \delta+\delta_{\text {spin }} \\
0 & \text { if } \quad j \delta+\delta_{\text {spin }}<t \leq(j+1) \delta
\end{array}\right.
\end{aligned}
$$

where $v_{\text {ins }}$ is the default insertion velocity of the needle, $j \in\{0,1, \ldots, \Delta / \delta\}$, and $\Delta / \delta$ is the total number of duty cycle intervals required to span the duration of each replanning step $(\Delta)$. This allows us to compute the control outputs required for actuation (insertion and rotation) of the needle.

Duty cycling requires that we characterize the maximum curvature $\left(\kappa_{0}\right)$ of the needle and determine the empirical relationship $(h(\kappa))$ between the curvature $(\kappa)$ and the duty cycling factor $(\alpha)$. We empirically determine that $h(\kappa)$ 
Chapter 2. Needle path planning and steering in a three-dimensional non-static environment using two-dimensional ultrasound images

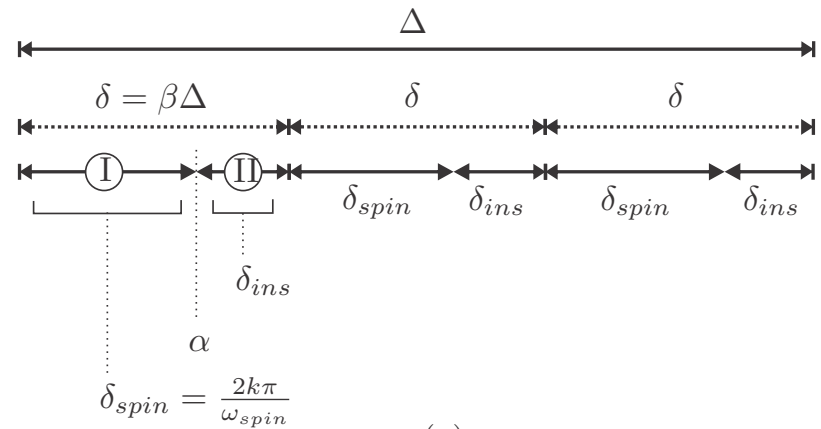

(a)

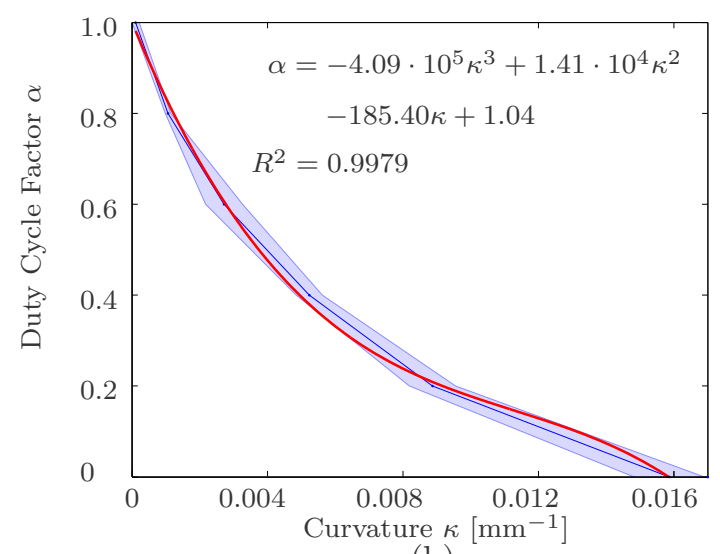

(b)

Figure 2.7: (a) The time duration $(\Delta)$ is split into multiple (e.g., three) intervals of duration $\delta$, each for $\beta=1 / 3$. Each interval is then composed of two intervals: (I) a spin interval of duration $\left(\delta_{\text {spin }}\right)$ given by $\delta_{\text {spin }}=\left(2 k \pi / \omega_{\text {spin }}\right), k \in \mathbb{Z}$ in which the needle is both inserted and rotated, and (II) an insertion interval of duration $\delta_{\text {ins }}$ in which the needle is only inserted without any rotation. (b) Characterization of the relationship $(\alpha=h(\kappa))$ for needle insertion in the soft-tissue phantom.

is dependent on the mechanical properties of the needle and the tissue and is not necessarily linear as demonstrated by prior work with duty cycled needle steering 24].

In order to estimate the relationship $(\alpha=h(\kappa)) \sqrt{2.9}$ for the soft-tissue phantom described in Section 4 , we performed repeated needle insertions up to $50 \mathrm{~mm}$. We varied the value of $\alpha$ between 0 and 1 in increments of 0.2 , and computed the duration of the duty cycling interval $(\delta)$ for a time interval $\Delta=$ 0.6 sec. Given a fixed insertion velocity $\left(v_{\text {ins }}=3[\mathrm{~mm} / \mathrm{s}]\right)$ and rotational velocity $\left(\omega_{\text {spin }}=5[\right.$ rotations $\left./ \mathrm{s}]\right)$, we command the actuators during each duty cycling interval with control outputs computed using (2.11). The application of these controls causes the needle tip to traverse a circular arc of some curvature $\kappa$ in a plane. 
In order to determine the effective curvature $(\kappa)$ of the planar arc, we recorded the needle tip pose $\left(\mathbf{H}_{\hat{t}}^{0}\right)$ after the end of each duty cycling interval for $N$ such intervals. We observed that the needle tip deviates from the plane because of initialization errors and other sources of uncertainty. To robustly estimate $\kappa$, we fit a circle to the set of $3 \mathrm{D}$ points given by $\mathbf{p}_{\hat{t}}^{0} \in \mathbb{R}^{3}, t=0, \ldots, N$. We accomplished this by first computing a best-fit plane that minimized the sum of the squared orthogonal distances from each point to the plane by performing principal component analysis (PCA) on the set of points. We then projected the points onto the first two principal components that span the plane and then fitted a circle to the set of projected $2 \mathrm{D}$ points using a robust circle fitting algorithm $[100]$. The curvature $(\kappa)$ was obtained by taking the reciprocal of the radius of this fitted circle. Fig. 2.7(b) shows the relationship $(\alpha=h(\kappa))$ for needle insertion in soft-tissue phantom used for our experiments. The needle achieved a maximum curvature $\kappa_{0}=0.016\left[\mathrm{~mm}^{-1}\right]$ (i.e., a bend radius of 62.5 [mm]) in the soft-tissue phantom.

We used the experimental measurements of $\alpha$ to compute a best-fit polynomial curve with a fixed maximum degree $(=3)$ that minimized the sum of the squared errors of the data points from the curve. This curve defines the relationship $(\alpha=h(\kappa))$. An important point to note is that the smaller the distance $\left(v_{\text {ins }} \delta\right)$ traveled by the needle tip in every duty cycling interval, the better the approximation of $\kappa$. We divided time duration $(\Delta)$ into a single duty cycling interval $(\delta=\beta \Delta$, with $\beta=1)$. This results in an insertion distance of $v_{\text {ins }} \delta=1.8[\mathrm{~mm}]$ per duty cycling interval.

Note, that the characterization $(\alpha=h(\kappa))$ used in motion planning and the duty cycle needle steering uses a single needle and tissue relationship. Motion planning and duty cycled needle steering in clinical practice should consider tissue properties of a wide range patients.

\subsection{Experiments}

In this section, we evaluate our 3D needle steering system described in Section 2.3. We experimentally evaluate the system in a soft-tissue phantom in different environments. In four experimental scenarios, we steer the needle to reach (moving) targets while avoiding (moving) obstacles.

\subsubsection{Experimental Setup and Materials}

In order to facilitate needle steering, all components of the experimental setup are integrated into a unified system (hardware and software for planning and ultrasound-guided control) in order to work together effectively. The experimental setup (Fig. 2.8 can be divided into two parts. First, the NID robotically inserts and rotates the needle about its axis. A telescopic sheath surrounds the needle to prevent buckling during insertion into the soft-tissue phantom. 
Chapter 2. Needle path planning and steering in a three-dimensional non-static environment using two-dimensional ultrasound images

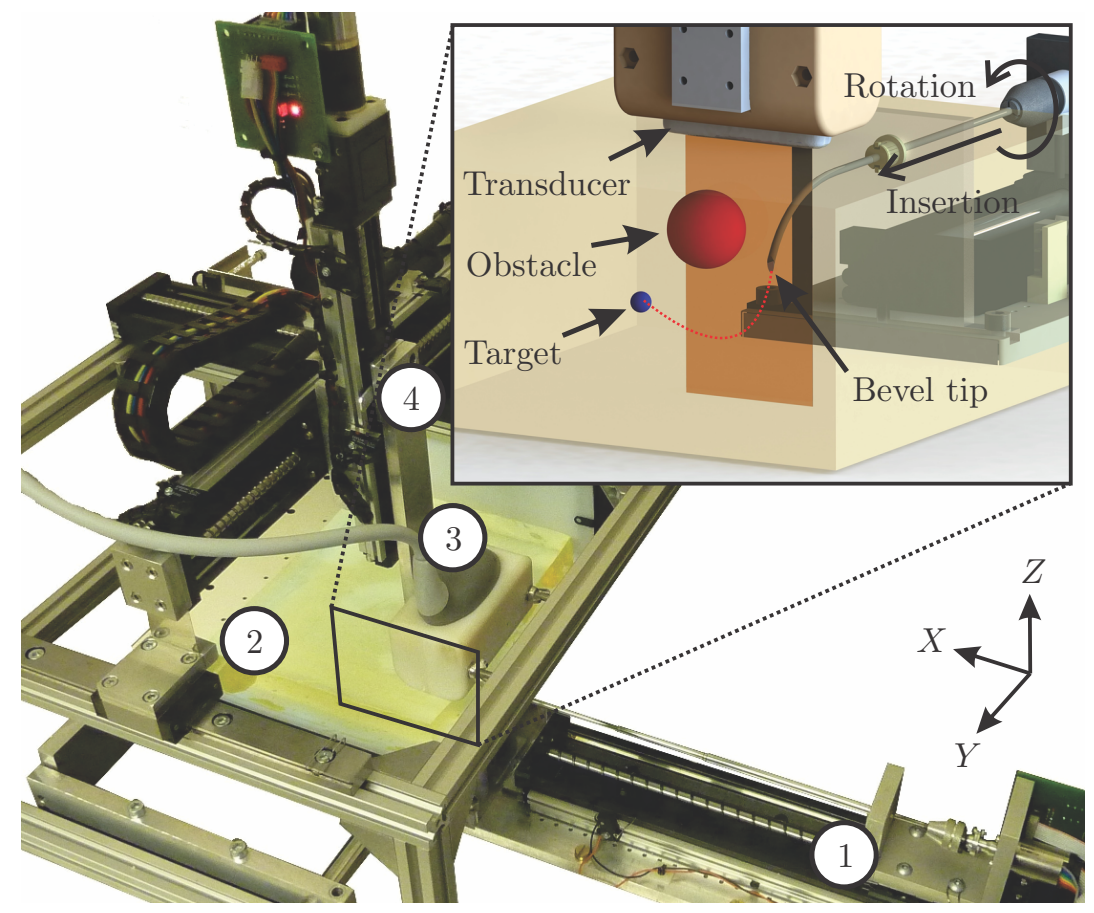

Figure 2.8: The experimental setup used to track and steer a flexible needle to reach a target while avoiding an obstacle. The needle, which is controlled at its base (inset) by a needle insertion device (1) is inserted into the soft-tissue phantom (2). The two-dimensional ultrasound transducer (3) is positioned at the needle tip during insertion by a transducer positioning device (4), which provides feedback for steering.

For the details of the NID we refer the reader to Roesthuis et al. and Van Veen et al. 30,101. Second, the transducer positioning device positions the $2 \mathrm{D}$ ultrasound transducer in $3 \mathrm{D}$ space. The positioning device consists of three orthogonally placed linear translation stages. The linear stages LX30, LX26 and LX20 (Misumi Group Inc., Tokyo, Japan) facilitate motion in $x$-, $y$ - and $z$ axes (frame $\left(\Psi_{0}\right)$ ) (Fig. 2.3), respectively. In order to actuate the linear stages, Maxon ECMax22 motors with GP32/22 gearheads (Maxon Motor, Sachseln, Switzerland) are used. The velocity of each stage is controlled by an Elmo Whistle 2.5/60 motor controller (Elmo Motion Control Ltd, Petach-Tikva, Israel). The positioning accuracies of the device are $27 \mu \mathrm{m}, 35 \mu \mathrm{m}$, and $41 \mu \mathrm{m}$ along the $x-, y$-, and $z$-axes, respectively. The ultrasound transducer is mounted to the positioning device end-effector using a perfectly fitting clamp.

The ultrasound images are obtained using an $18 \mathrm{MHz}$ transducer connected to a Siemens Acuson S2000 ultrasound machine (Siemens AG, Erlangen, Germany). The ultrasound machine is linked to a computer using an S-video cable that transfers the images $(720 \times 576$ pixels $)$ with a frame rate of 25 frames per 


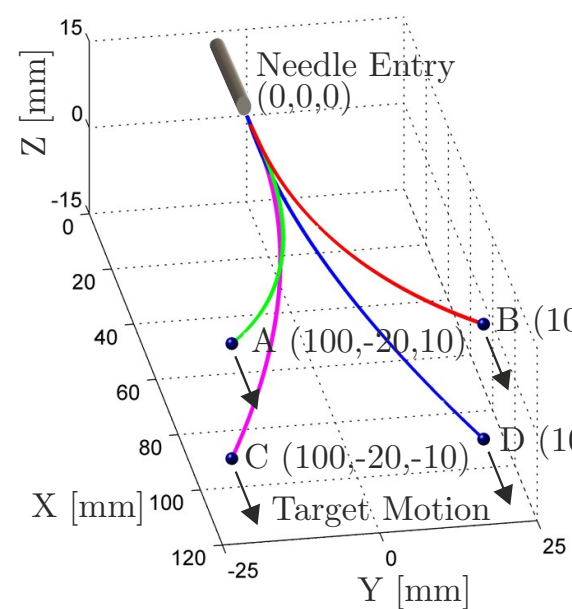

(a)

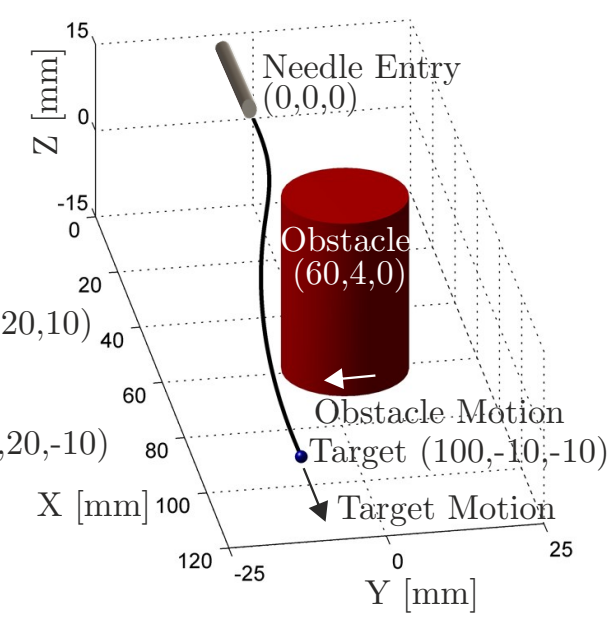

(b)

Figure 2.9: Experimental needle steering scenarios: (a) The needle is steered to a moving spherical target at four different locations: A, B, C and D in Scenarios I-A, -B, -C and $\mathrm{D}$, respectively. (b) The needle is steered around a cylindrical-shaped obstacle (e.g., bone) which is in the direct path of the needle to reach a spherical target (Scenarios II-IV). In Scenario II, the needle is steered to reach a stationary target while avoiding a stationary obstacle. In Scenario III, the needle is steered to reach a moving target while avoiding a stationary obstacle. In Scenario IV, the needle is steered to reach a target in an environment with obstacle and target motion.

second. The ultrasound images are used to track the needle during insertion into a soft-tissue phantom. The soft-tissue phantom is obtained by a weight based mixture of $84.1 \%$ water, $14.9 \%$ gelatin powder (Dr.Oetker, Ede, The Netherlands) and 1.0\% silica gel 63 (E. Merck, Darmstadt, Germany). The elasticity of the gelatin mixture used to mimic human breast tissue is $35 \mathrm{kPa}$ (Young's Modulus) [102]. Silica gel is added to the mixture to simulate the acoustic scattering of human tissue in ultrasound images 103 . The flexible needle, which is a solid wire, is made of Nitinol alloy (nickel and titanium). The Nitinol needle has a diameter of $0.5 \mathrm{~mm}$ with a bevel angle (at the tip) of $30^{\circ}$.

\subsubsection{Experimental Scenarios}

To evaluate the performance of our system that integrates ultrasound-based needle tracking and motion planning algorithms, we conducted experiments in which our system steers the needle to a target (e.g., lesion) in 3D non-static environments while avoiding obstacles. We evaluate our system's targeting accuracy in four experimental scenarios (Fig. 2.9). We execute our system ten 
Chapter 2. Needle path planning and steering in a three-dimensional non-static environment using two-dimensional ultrasound images

times for each experimental scenario. The needle insertion and rotational velocities used during the experiments are $3[\mathrm{~mm} / \mathrm{s}]$ and 5 [rotations/s], respectively. In our experiments we used virtual obstacles and targets. The target and obstacle locations are expressed in $(x[\mathrm{~mm}], y[\mathrm{~mm}], z[\mathrm{~mm}])$-coordinates of the fixed reference (frame $\left(\Psi_{0}\right)$ ).

- In Scenarios I-A, -B, -C and -D, the needle is steered to reach a moving target at four different locations (A, B, C and D), respectively.

- In Scenario II, the needle is steered to reach a stationary target while avoiding a stationary obstacle.

- In Scenario III, the needle is steered to reach a moving target while avoiding a stationary obstacle.

- In Scenario IV, the needle is steered to reach a moving target while avoiding a moving obstacle.

The initial target locations for experimental Scenarios I-A, -B, -C and -D are $(100,-20,10),(100,20,10),(100,-20,-10)$ and $(100,20,-10)$, respectively. The initial target location for experimental Scenarios II-IV is $(100,-10,-10)$. The target depth of $100 \mathrm{~mm}$ is within the range of typical biopsy depths of lesions and tumors (retroperitoneal) which are approximately $35-115 \mathrm{~mm}[104$. The target size is $2 \mathrm{~mm}$, which is currently the smallest detectable size of a lesion in a breast mammography [105]. Target motion is simulated in Scenarios I, III and IV to investigate the effects of target motion on the needle steering accuracy. During insertion, the needle exerts a force on the target (in the positive $x$-axis of frame $\left(\Psi_{0}\right)$ ), which causes target motion. After 20 seconds of needle insertion, the target starts to move with a constant velocity of $0.4 \mathrm{~mm} / \mathrm{s}$ in the positive $x$-axis (frame $\left(\Psi_{0}\right)$ ) until the needle reached the target. The simulated target motion results in a displacement of approximately $7.0 \mathrm{~mm}$, which is commonly observed during clinical interventions in breast tissue 85 88 106.

In order to evaluate the maneuverability of the needle during steering, an obstacle is positioned in the direct path of the needle to reach the target (Scenarios II-IV). The proposed obstacle has a cylindrical shape (e.g., bone) with a $20 \mathrm{~mm}$ diameter, and is located (center of the cylinder) at $(60,4,0)$. Obstacle motion is introduced in Scenario IV to simulate the non-static behavior of organs which constantly move. During the first 10 seconds of needle insertion, the obstacle moves with a constant velocity of $0.3 \mathrm{~mm} / \mathrm{s}$ in the negative $y$-axis (frame $\left(\Psi_{0}\right)$ ) (towards the needle path). After 10 seconds, obstacle motion is stopped and a total displacement of $3 \mathrm{~mm}$ is achieved.

\subsubsection{Experimental Results}

The experimental results of Scenarios I-IV are provided in Table 2.1. A representative result for each of the Scenarios I(-A, -B, -C and -D), II, III and IV 


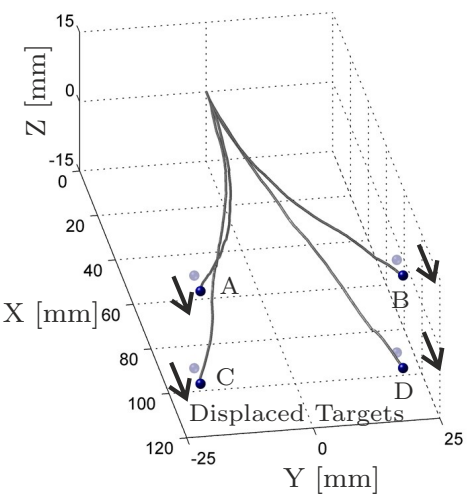

(a)

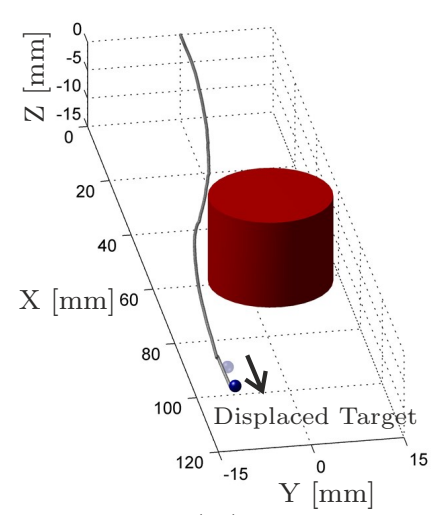

( c)

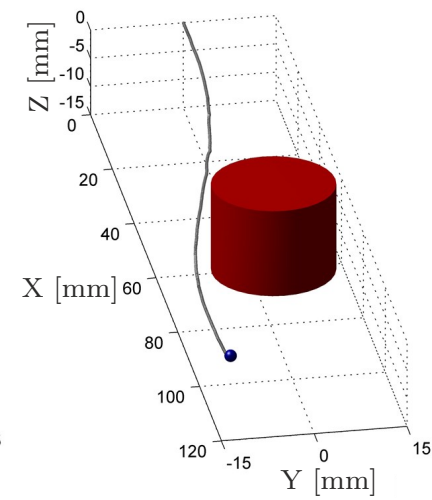

(b)

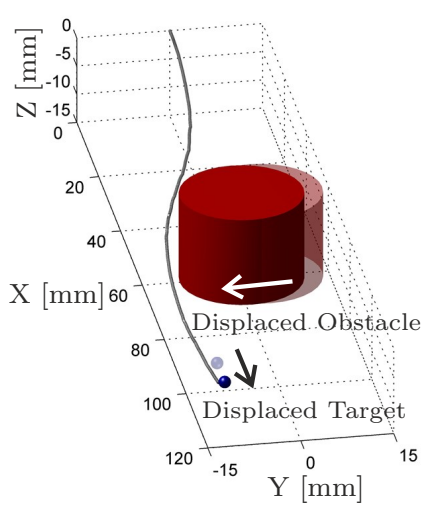

(d)

Figure 2.10: Representative experimental needle steering results and mean absolute errors (MAE) in targeting accuracy: (a) Scenario I (moving target) - MAE: (I-A) $0.59 \mathrm{~mm}$, (IB) $0.54 \mathrm{~mm}$, (I-C) $0.86 \mathrm{~mm}$ and (I-D) $0.82 \mathrm{~mm}$. (b) Scenario II (both stationary obstacle and target) - MAE: $1.76 \mathrm{~mm}$. (c) Scenario III (stationary obstacle and moving target) MAE: $1.33 \mathrm{~mm}$. (d) Scenario IV (both moving obstacle and target) - MAE: $2.16 \mathrm{~mm}$. The initial position of the cylindrical obstacle (bone) is shaded red, while its end position is solid red. The shaded blue sphere represents the initial target location, while the final target location is represented by a solid blue sphere.

are given in Fig. 2.10(a), (b) (c) and (d), respectively. After the needle is steered to reach the target, its final estimated needle tip location from ultrasound tracking is evaluated with respect to the target location. The mean absolute errors (MAE) with standard deviations in the needle tip position with respect to the target along $x-\left(\epsilon_{x}\right)-, y$ - $\left(\epsilon_{y}\right)$ - and $z-\left(\epsilon_{z}\right)$-axes (frame $\left(\Psi_{0}\right)$ ) are reported. The MAE are determined in order to evaluate the distance between tip and target. Experimental results of Scenarios I-A, -B, -C and -D show MAE 
Chapter 2. Needle path planning and steering in a three-dimensional non-static environment using two-dimensional ultrasound images

Table 2.1: Targeting errors for ultrasound needle tracking and steering experiments (Scenarios I-IV). Mean absolute errors (MAE) for needle tip targeting along $x-\left(\epsilon_{x}\right)-, y$ - $\left(\epsilon_{y}\right)$ and $z$ - $\left(\epsilon_{z}\right)$-axes (frame $\left(\Psi_{0}\right)$ ) are provided. The targeting errors are evaluated using the estimated needle tip pose from ultrasound tracking. For each scenario, MAE for targeting accuracy are evaluated. Each experiment is conducted ten times.

\begin{tabular}{cccccc}
\hline \multicolumn{2}{c}{ Scenario } & $\begin{array}{c}\epsilon_{x} \\
{[\mathrm{~mm}]}\end{array}$ & $\begin{array}{c}\epsilon_{y} \\
{[\mathrm{~mm}]}\end{array}$ & $\begin{array}{c}\epsilon_{z} \\
{[\mathrm{~mm}]}\end{array}$ & $\begin{array}{c}\text { MAE } \\
{[\mathrm{mm}]}\end{array}$ \\
\hline \multirow{3}{*}{$\mathrm{I}$} & $\mathrm{A}$ & $0.06 \pm 0.02$ & $0.18 \pm 0.13$ & $0.51 \pm 0.37$ & $0.59 \pm 0.30$ \\
\cline { 2 - 6 } & $\mathrm{B}$ & $0.05 \pm 0.03$ & $0.46 \pm 0.35$ & $0.21 \pm 0.27$ & $0.54 \pm 0.39$ \\
\cline { 2 - 6 } & $\mathrm{C}$ & $0.06 \pm 0.03$ & $0.66 \pm 0.37$ & $0.41 \pm 0.35$ & $0.86 \pm 0.35$ \\
\cline { 2 - 6 } & $\mathrm{D}$ & $0.05 \pm 0.03$ & $0.53 \pm 0.40$ & $0.57 \pm 0.44$ & $0.82 \pm 0.52$ \\
\hline $\mathrm{II}$ & & $0.05 \pm 0.03$ & $1.63 \pm 1.06$ & $0.53 \pm 0.66$ & $1.76 \pm 1.02$ \\
\hline $\mathrm{III}$ & & $0.05 \pm 0.04$ & $1.11 \pm 0.55$ & $0.54 \pm 0.71$ & $1.33 \pm 0.47$ \\
\hline $\mathrm{IV}$ & & $0.06 \pm 0.03$ & $1.37 \pm 0.68$ & $1.56 \pm 0.82$ & $2.16 \pm 0.88$ \\
\hline
\end{tabular}

of $0.59 \mathrm{~mm}, 0.54 \mathrm{~mm}, 0.86 \mathrm{~mm}$ and $0.82 \mathrm{~mm}$, respectively. In Scenario II, an MAE of $1.76 \mathrm{~mm}$ is observed. For Scenario III, an MAE of $1.33 \mathrm{~mm}$ is reported, while for Scenario IV, an MAE of $2.16 \mathrm{~mm}$ is observed.

The experimental results of Scenarios I(-A, -B, -C and -D) show targeting errors less than $1 \mathrm{~mm}$. Note, that the smallest detectable lesions in breast mammography are reported to be $2 \mathrm{~mm}$, with most lesions close to $11 \mathrm{~mm}$ in diameter [105, 107]. Therefore, the proposed tracking and steering method is capable of targeting the smallest detectable lesions in scenarios without obstacles in applications such as breast biopsy. We observe that absence of an obstacle (Scenarios I(-A, -B, -C and -D)) results in better targeting accuracy as compared to the other scenarios. This could be attributed to the fact that once the needle is steered around the obstacle according to a computed motion plan (Scenarios II-IV), the maneuverability of the needle and its ability to correct for perturbations is constrained. Hence, the range of feasible motion plans to the target diminishes due to the obstacle. Further, the decrease in performance (Scenarios II-IV) may be due to torsional flexibility of the needle, which results in needle tip pose estimation $\left(\mathbf{H}_{\hat{t}}^{0}\right)$ errors.

We observe better targeting accuracy in Scenario III (stationary obstacle and moving target) as compared to Scenario II (both stationary obstacle and target). A possible explanation is that once the needle is steered around the obstacle, the motion planner has more options and more time to correct for uncertainties when the target moves away from the obstacle. However, as observed in Scenario IV, the presence of obstacle motion results in decreased targeting accuracy. This can be explained by the reduced range of motion plans 
which are feasible due to the substantial target and obstacle motion, especially when obstacle motion reduces the space of feasible paths that reach the target. Nonetheless, even in the challenging scenario of target and obstacle motion, our method still achieves errors that are typically at or below $2.16 \mathrm{~mm}$.

Experienced clinicians inserting radioactive seeds into the prostate gland for brachytherapy prostate cancer treatment experience average placement errors of $6.3 \mathrm{~mm}$, about $15 \%$ of the prostate's diameter [108]. Further, a study by Blumenfeld et al. concluded that prostate biopsies using rigid needles performed by experienced clinicians show average targeting errors between 5.5-6.5 mm 109]. We evaluate our system using soft-tissue phantoms which leads to several simplifications as opposed to studies in biological tissue. Nonetheless, our method demonstrates needle steering that appears comparable to an experienced clinician in terms of accuracy while providing new obstacle avoidance capabilities. This indicates that our system, with further development, is on track to be applicable to wide class of clinical applications.

\subsection{Conclusions and Future Work}

We present and evaluate a needle steering system capable of autonomously and accurately guiding a steerable needle to a target in a non-static 3D environment using 2D ultrasound images. Our system achieves high accuracy in non-static environments by effectively integrating two major components: a safe, ultrasound-based tracker of the needle pose and a fast needle motion planner that reacts to perturbations in target and obstacle locations. To accurately track the needle tip, we use a clinically-available 2D ultrasound transducer which is orientated perpendicular to the direction of insertion and does not require that the procedure be performed in a single plane. The system estimates the needle tip pose by controlling the position of the ultrasound transducer to obtain images at the the needle tip and using image processing algorithms. The needle tip pose is used in motion planning to compute feasible paths that reach a target while avoiding an obstacle. The planner is sufficiently fast such that the system can repeatedly execute it as new tip pose estimates are obtained from the ultrasound tracker, enabling the system to compensate for uncertainties in steering and to correct for perturbations in obstacle and target locations. In experiments in which the targets moves approximately $7 \mathrm{~mm}$ over the course of the procedure (which is typical in breast biopsies), the system achieves low MAE of $0.86 \mathrm{~mm}$ (without obstacles) and $2.16 \mathrm{~mm}$ (with a moving obstacle).

In future work, we will build on this system and investigate additional avenues to reduce needle placement errors in non-static tissue environments while avoiding anatomical obstacles. We plan to investigate the effects of variations in tissue elasticity on needle curvature (the empirical relationship $\alpha=h(\kappa)$ in Section 2.3.5 and to incorporate methods that compensate for torsional flexibility of the needle. Further, we will also investigate improving targeting 
Chapter 2. Needle path planning and steering in a three-dimensional non-static environment using two-dimensional ultrasound images

accuracy and reducing tissue damage by integrating different needle steering methods with motion planning [55]. In order to provide a more realistic testing scenario, needle steering in biological tissue, including tracking of real targets, will be investigated. Our ultrasound-based tip tracking algorithm evaluates the needle location that is affected by CTA. Some preliminary research indicates that artifacts such as CTA are also observed in biological tissue, although effort is required to properly correct for distortions such as warping that are often observed in ultrasound images of biological tissue. So with some modifications, we believe our proposed ultrasound-guided tracking and control method would be applicable to biological tissue. In addition to experiments in biological tissue, advancements in instrument design need to be made to enable biopsies with flexible needles. Further, we are currently adapting our transducer positioning device to move on curved surfaces using additional degrees of freedom and a force sensor to maintain transducer-tissue contact. While we aim to continue to improve the system, our current study demonstrates the feasibility and potential of tracking and steering flexible needles, which has clinical applications for procedures such as breast and prostate biopsies and brachytherapy. 


\section{Part II}

\section{Continuum robots: Catheter steering}





\section{Preface}

\section{Continuum robots: Catheter steering}

In Part II, the closed-loop control method for continuum robots is expanded to delivery sheaths and steerable catheters used in cardiovascular interventions. The ultrasound tracking method described in Chapter 2 is adopted for online tracking of delivery sheaths and steerable catheters. Furthermore, cardiovascular applications such as degenerative aortic and mitral valve diseases are considered. In addition to accurate continuum robot tip positioning, instrument tip stabilization for beating heart motions is considered. In Chapter 3, a robotically-actuated delivery sheath is controlled in closed loop by using a predictive controller. In experiments, accurate delivery sheath tip positioning and stabilization for beating heart aortic valve motions is demonstrated. Furthermore, constraints are integrated in the control strategy, which are used to restrict the instrument motion to an allowable region. The aim is to demonstrate the ability of the closed-loop control method to avoid sensitive tissue, which could be present in surgery. In Chapter 4, a closed-loop control method for a steerable and flexible catheters is presented. A catheter steering module is developed and used to stabilize the instrument tip for beating heart mitral valve motions. In closed-loop control, feedback of the catheter is provided by ultrasound images or electromagnetic tracking sensors embedded in the instrument base and tip. Closed-loop control experiments are then performed in order to demonstrate catheter tip stabilization for beating heart motions in a realistic and functional mitral valve model. In Chapter 5, an improved beating heart testbed for the evaluation of continuum robots in cardiovascular interventions is described. The beating heart testbed is developed in order to evaluate various continuum robot designs in a clinically-relevant environment. The beating heart testbed considers beating heart motions, systemic circulation and realistic anatomy with functional heart valve models. 


\title{
CHAPTER 3 \\ Model predictive control of a robotically-actuated delivery sheath for beating heart compensation
}

\begin{abstract}
Minimally invasive surgery (MIS) during cardiovascular interventions reduces trauma and enables treatment to high-risk patients who were initially denied surgery. However, restricted access, reduced visibility and control of the instrument at the treatment locations limits the performance and capabilities of such interventions during MIS. Therefore, the demand for technology such as steerable sheaths or catheters that assists the clinician during the procedure increases. In this study, we present and evaluate a robotically-actuated delivery sheath (RADS) capable of autonomously and accurately compensating for beating heart motions by using a model-predictive control (MPC) strategy. We develop kinematic models and present on-line ultrasound segmentation of the RADS that are integrated with the MPC strategy. As a case study, we use pre-operative ultrasound images from a patient to extract motion profiles of the aortic heart valve (AHV). This allows the MPC strategy to anticipate for AHV motions. Further, mechanical hysteresis in the steering mechanism is compensated for in order to improve tip positioning accuracy. The novel integrated system is capable of controlling the articulating tip of the RADS to assist the clinician during cardiovascular surgery. Experiments demonstrate that the RADS follows the AHV motion with a mean positioning error of $1.68 \mathrm{~mm}$. The presented modeling, imaging and control framework could be adapted and applied to a range of continuum-style robots and catheters for various cardiovascular interventions.
\end{abstract}

This chapter has been previously published as

[110 G.J. Vrooijink, A. Denasi, J.G. Grandjean, and S. Misra, Model predictive control of a robotically-actuated delivery sheath for beating heart compensation, International Journal of Robotics Research, 36(2): 193209, April 2017.

while preliminary work is described in

111 G.J. Vrooijink, T.T.M. Ellenbroek, P. Breedveld, J.G. Grandjean, and S. Misra, A preliminary study on using a robotically-actuated delivery sheath (RADS) for transapical aortic valve implantation, in Proceedings of the IEEE International Conference on Robotics and Automation (ICRA), Hong Kong, China, pages 4380-4386, May-June 2014. 
Chapter 3. Model predictive control of a robotically-actuated delivery sheath for beating heart compensation

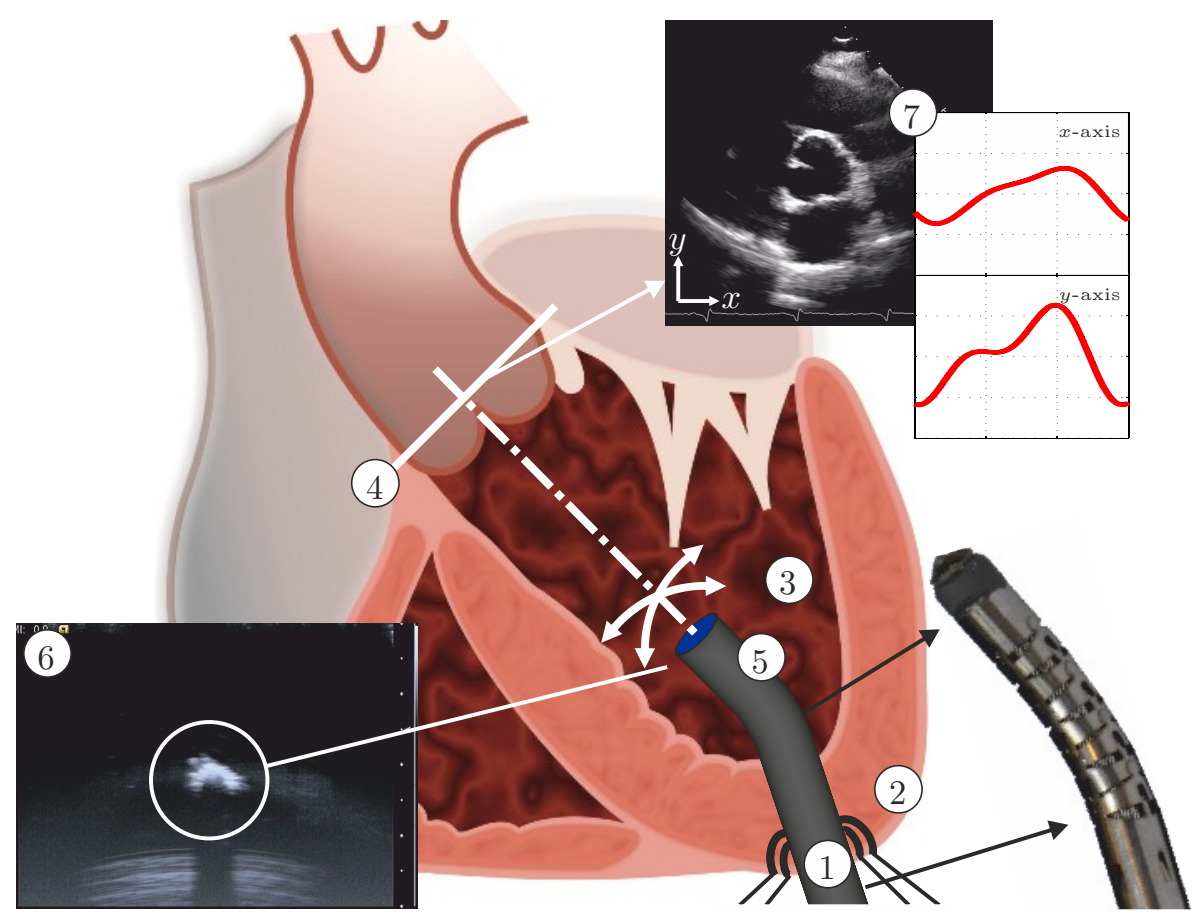

Figure 3.1: Model predicitive control (MPC) can be used to steer the robotically-actuated delivery sheath (RADS) in order to assist the clinician during cardiovascular surgery. The potential of MPC in cardiovascular surgery can be demonstrated by compensating for the aortic heart valve $(\mathrm{AHV})$ motion in a representative case of transapical transcatheter aortic valve implantation. The RADS (1) is inserted through the apex (2) into the left ventricle (3) and oriented towards to the aortic annulus (4). The articulating tip (5) of the RADS can be steered inside the left ventricle under ultrasound (6) image-guidance in two degrees-offreedom by two pairs of antagonistically-configured tension wires. Pre-operative ultrasound data (7) can be used as an input to the MPC strategy to anticipate and compensate the AHV motion during surgery.

\subsection{Introduction}

According to the World Health Organization, cardiovascular diseases are among the leading causes of death globally [112]. Technological developments have the ability to improve and enable treatment of cardiovascular diseases $[1-3]$. Traditionally, open surgery via sternotomy is performed to gain surgical access to the heart, while a heart-lung machine provides life support. Accessibility to the heart is considered to be a major advantage during the open surgery, which is at the expense of severe patient trauma. As an alternative, minimally invasive surgery (MIS) could reduce trauma and enable treatment to high-risk patients who were initially denied surgery [1, 9, 14, 16, 113]. However, limited access, vision and control of the instrument at the treatment locations impedes 
the performance of MIS. During interventions performed without a heart-lung machine, often beating heart motion compensation is desired and could potentially enable future cardiovascular interventions. Tracking of the beating heart requires attention of the surgeon, whose accuracy deteriorates for complex repetitive motions up to 60 beats-per-minute (BPM) [19]. Therefore, the demand for technology that assists the clinician via shared control during the procedure increases.

In this study, we focus on a model predictive control (MPC) strategy that anticipates and compensates for the beating heart motion (Fig. 3.1). The MPC strategy is used to compensate the beating heart motion by using a roboticallyactuated delivery sheath (RADS). Another advantage of MPC is the ability to integrate motion constraints on the instrument in order to prevent damage to sensitive tissue during the procedure. As a case study, we use transcatheter aortic valve implantation (TAVI) to demonstrate the potential of our MPC strategy. Further, applications such as ablation, valve repair surgery and mitral valve chordal replacement can potentially benefit from such an MPC strategy. TAVI can be performed via the transfemoral (TF) or transapical (TA) routes. In this case study, we focus on the TAVI-TA approach, which provides direct surgical access to the aortic and mitral heart valves 9,10 .

\subsubsection{Related Work}

Recent studies demonstrate capabilities of MIS in cardiovascular applications such as angioplasty and patent foramen ovale 69, 80]. These studies describe the use of robotic instruments in order to enable MIS. However, MIS in cardiovascular applications such as coronary artery bypass, ablation and valve surgery could significantly benefit from beating heart compensation. Active robotic stabilization at the treatment location could provide a virtually-still scenario that allows the clinician to perform the primary task of the procedure as if the heart was stopped. Compensation of beating heart motions has been extensively covered in research. Studies that focused on the beating heart surface have been described $70,74,76,114$. These studies cover various predictive strategies by filtering and control to anticipate the beating heart motions. 73 reported significant correlations between beating heart motions and electrocardiogram (ECG) signals, which were utilized for synchronization by 74 . The majority of these studies used predictive strategies to improve accuracy and to anticipate for occlusions in endoscopic cameras images, while this study focusses on ultrasound-guided repair surgery in the beating heart.

Cardiovascular procedures such as ablation and valve-implantation or repair surgery are often assisted by two-dimensional (2D) or three-dimensional (3D) ultrasound, which are used to evaluate cardiac functionalities (e.g., valve closing) 2. The presence of ultrasound imaging during the procedure provides the potential for on-line soft tissue and instrument tracking. 75] evaluated predictive filtering to enable ultrasound-guided robotic tracking of cardiac mo- 
Chapter 3. Model predictive control of a robotically-actuated delivery sheath for beating heart compensation

tions for potential use in ablation. A study by [78], demonstrated capabilities of applying a constant force against a moving target by using ultrasound image feedback and force sensing. Whereas [81] focused on predictive control using an ultrasound-guided tele-operated robot to anticipate for cardiac motions. All of these methods describe one dimensional motion compensation, which is along the lateral axis of the instrument. However, existing applications in valve implantation and repair surgery such as mitraclip placement could benefit from motion compensation in two or more degrees-of-freedom (DOF) $3,9,17$. Further, beating heart motion compensation in two or more DOF could potentially enable future applications in minimally invasive cardiovascular surgery. In this study, we cover an integrated system capable of autonomously and accurately compensating for beating heart motions in 2D by using a MPC strategy. Further, our MPC strategy guided by ultrasound imaging considers active constraint handling in order to avoid damage to sensitive tissue. Major aspects in MPC of the integrated system are kinematic modeling of continuum-style robots and feedback on its pose.

Kinematic modeling is used to describe the RADS shape and articulating tip motion. Modeling of continuum-style robots have been investigated by several groups $27,31,58,62,63,65,67,68,115,116$. Various continuum-style robotic instruments with potential in cardiovascular applications have been described in literature. A large number of the continuum-style robots are actuated by push-and-pull systems or tendon mechanisms. We describe the kinematics of the tendon-driven constant-curvature RADS using both robot-specific and -independent submappings 31. The kinematics presented in this study considers a rigid link which is attached to the flexible segment of the RADS. However, modeling mismatches and disturbances acting on the system affect the tip positioning performance of continuum-style robots.

Tracking of continuum-style robots plays an important role in order to reduce tracking errors caused by modeling mismatches and external disturbances. In TAVI, the clinician is often assisted by 2D and 3D transesophageal or transthoracic echocardiography [2]. 2D ultrasound has been used for 2D and $3 \mathrm{D}$ tracking of flexible instruments by several groups $32,40,41,45,111$. 3D ultrasound-based tracking of cardiac catheters (some equipped with markers) have also been described in literature $39,44,79,117]$. In this study, we integrate an on-line and a robust tracking algorithm that uses 2D ultrasound images of the RADS.

\subsubsection{Contributions}

In this study, we demonstrate an integrated system that assists the clinician by compensating for beating heart motions, which could be used in existing and potential future cardiovascular interventions. By active stabilization of the instrument in the beating heart, a virtually-still treatment location could be provided. This allows the clinician to perform the procedure as if the heart 
was stopped. Such a system requires instrument modeling and tracking, which is integrated in a control strategy capable of anticipating for beating heart motions. In this study, we incorporate the forward, inverse and differential kinematic models of the RADS, which considers a rigid link or a tool attached to the flexible tip segment. Subsequently, we integrate a robust 2D ultrasound image segmentation algorithm capable of on-line evaluation of the RADS tip position. Furthermore, we provide the control objective and corresponding MPC strategy, which considers kinematic modeling and ultrasound feedback. The MPC strategy anticipates for beating heart motions by incorporating models based on pre-operative patient data. In addition to the aforementioned contributions, the MPC strategy considers active constraint handling in order to prevent damage to sensitive tissue. To the best of our knowledge, we are the first to present an ultrasound-guided MPC strategy capable of compensation for beating heart motions in 2D using a RADS. As a case study, we focus on TAVI-TA by integrating an aortic heart valve (AHV) model in the MPC strategy, which is based on pre-operative $2 \mathrm{D}$ ultrasound patient images that describes the AHV motion during the cardiac cycle. The presented strategy for beating heart compensation could be applicable to a wide variety of existing and potential future cardiovascular interventions. Further, we improved the RADS tip positioning accuracy by compensating for instrument hysteresis. Three experimental scenarios using a water container setup demonstrate MPC of the novel integrated system that autonomously and accurately compensates for AHV motions.

\subsection{Methods}

This section presents techniques to enable tracking and MPC-based steering of the RADS. Details on the design of the RADS are provided by 111]. We summarize the forward, inverse and differential kinematics which are used in MPC. Furthermore, we describe the 2D ultrasound segmentation method of the RADS used as feedback for MPC. The MPC strategy includes modeling of the heart valve motion based on pre-operative $2 \mathrm{D}$ ultrasound data. By combining the kinematic modeling and segmentation of the RADS with MPC, the system is able to anticipate the beating heart motion. Note, in the derivations presented, for notational simplicity, we leave out the discrete time variable denoted by $k$ (except where needed for clarity). Further, a list of symbols describing the variables used in this study is provided in the Nomenclature of this chapter (page 79). 
Chapter 3. Model predictive control of a robotically-actuated delivery sheath for beating heart compensation

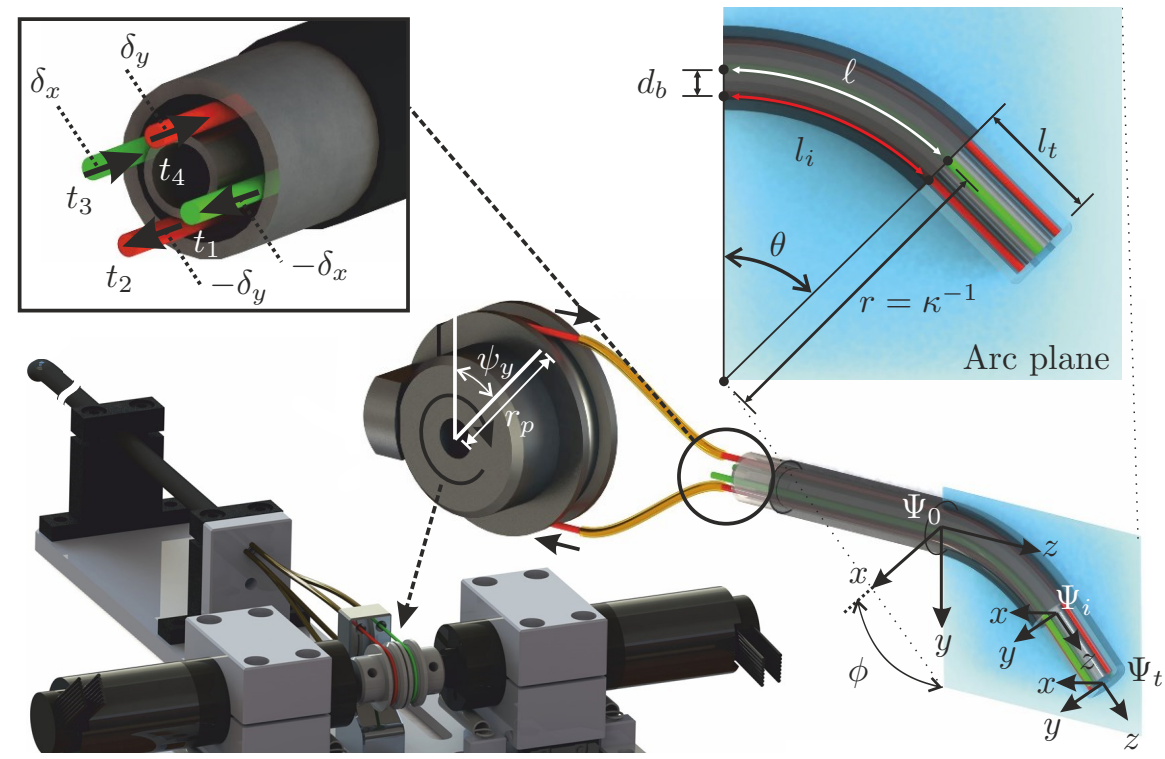

Figure 3.2: An overview of the robotically-actuated delivery sheath (RADS). The articulating tip of the RADS is actuated in two degrees-of-freedom by two pairs (red and green) of antagonistic-configured tension wires driven by a two pulleys with radii $\left(r_{p}\right)$ and angles $\left(\psi_{x}\right.$ and $\psi_{y}$ ). Three coordinate systems are assigned to describe the tip pose of the RADS: $\Psi_{0}$ is the reference frame fixed to the shaft, $\Psi_{i}$ is the intermediate frame assigned to the arc section and frame $\left(\Psi_{t}\right)$ is fixed to the articulating tip. Displacement of the tension wires $\left(t_{1}, \cdots, t_{4}\right)$ by $\delta_{x}$ and $\delta_{y}$ (inset centre) results in instrument bending along the $x$ - and $y$-axes (frame $\left(\Psi_{0}\right)$ ), respectively. The arc of the RADS with parameters, bend angle $(\theta)$, backbone length $(\ell)$, radius $(r)$ and curvature $(\kappa)$ lies in a plane described by the arc plane (inset right). The orientation of the arc plane about the $z$-axis of the reference frame $\left(\Psi_{0}\right)$ is denoted by angle $(\phi)$. Further, the tendon distance to the backbone arc $(\ell)$ is denoted $d_{b}$. A rigid link (not completely shown) of length $\left(l_{t}\right)$ is attached to the arc (frame $\left(\Psi_{i}\right)$ ) of the RADS.

\subsubsection{Instrument modeling}

\subsubsection{Forward kinematics}

The design and integration of the tendon-driven RADS used in this study is shown in Fig. 3.2. Two pairs of antagonistically-configured tension wires are used to actuate the articulating tip of the RADS in 2DOF. Each pair of wires is actuated by a single pulley and controls tip movement in a single DOF (Fig. 3.2). The four-wire design allows for tip movement in two DOF by using two actuators instead of three, that are required in an instrument with three wires. A kinematic model of the tendon-driven continuum-style robot can be described by a robot-specific and a robot-independent submapping [31. The robot-specific mapping relates the actuator-space to the configuration-space. The actuator-space is given by the angles of the pulleys $\left(\psi_{x}\right.$ and $\left.\psi_{y}\right)$, while the 
configuration-space is described by arc parameters such as arc curvature $(\kappa)$, arc plane angle $(\phi)$ and arc length $(\ell)$ ). The robot-independent mapping transforms the configuration-space to the task space (intermediate frame $\left(\Psi_{i}\right)$ ).

In order to evaluate the arc parameters of the configuration-space, the relation between tendon manipulation at the base and the resulting arc needs to be described. In the derivation presented, we denote for notational simplicity: $c_{*}=\cos (*)$ and $s_{*}=\sin (*)$. The RADS used in this study is actuated using four tendons $\left(t_{i}\right)$, where $(i=1, \cdots, 4)$ with corresponding tendon lengths $\left(l_{i}\right)$ as shown in Fig. 3.2 (inset centre). The relationship between the arc length of the RADS $(\ell)$ and the arc length of a single tendon $\left(l_{i}\right)$ is given by

$$
\ell=l_{i}+\theta d_{b} c_{\phi_{i}}
$$

where, $\theta$ is the bend angle, $d_{b}$ denotes the distance between the backbone and tendon and $\phi_{i}$ describes the angle between the bending direction of the RADS and the location of a single tendon $\left(t_{i}\right)$. Note, that the distance $\left(d_{b}\right)$ in our instrument is equal for all tendons (Fig. 3.2). Further, the bend angle $(\theta)$ is related to the curvature by $\theta=\kappa \ell$.

Given the tendon configuration as illustrated in Fig. 3.2, we can formulate the individual tendon angles by $c_{\phi_{1}}=c_{\phi}, c_{\phi_{2}}=s_{\phi}, c_{\phi_{3}}=-c_{\phi}$ and $c_{\phi_{4}}=$ $-s_{\phi}$. By combining the individual tendon angles with (3.1), we can obtain an expression between the arc length $(\ell)$ of the RADS and the individual tendon lengths $\left(l_{i}\right)$, which yields

$$
\ell=\frac{l_{1}+l_{2}+l_{3}+l_{4}}{4}
$$

The antagonistic configuration allows for pairing of the tendons described by $t_{1}$ and $t_{3}$, and $t_{2}$ and $t_{4}$. By using the tendon pairs and 3.2 , the arc plane angle $(\phi)$ is obtained according to

$$
\phi=\operatorname{atan} 2\left(l_{4}-l_{2}, l_{3}-l_{1}\right)
$$

while the curvature is evaluated as

$$
\kappa=\frac{\left.\left(l_{1}-3 l_{2}+l_{3}+l_{4}\right) \sqrt{\left(l_{4}-l_{2}\right)^{2}+\left(l_{3}-l_{1}\right)^{2}}\right)}{d_{b}\left(l_{1}+l_{2}+l_{3}+l_{4}\right)\left(l_{4}-l_{2}\right)} .
$$

Note, that the arc parameters $(\ell, \phi$ and $\kappa)$ described in $(3.2)-(3.4)$ are functions of the individual tendon lengths $\left(l_{i}\right)$. These individual tendon lengths $\left(l_{i}\right)$ are manipulated by displacements $\left(\delta_{x}\right)$ and $\left(\delta_{y}\right)$, which are introduced by two actuated pulleys (Fig. 3.2). The relation between tendon displacements $\left(\delta_{x}\right.$ and $\left.\delta_{y}\right)$ and pulley angles $\left(\psi_{x}\right.$ and $\left.\psi_{y}\right)$ are given by $\delta_{x}=r_{p} \psi_{x}$ and $\delta_{y}=r_{p} \psi_{y}$, where $r_{p}$ describes the pulley radius (equal radii for both pulleys). Hence, we can use (3.1) to formulate an expression for all tendon lengths $\left(l_{i}\right)$ as a function of the pulley angles $\left(\psi_{x}\right.$ and $\left.\psi_{y}\right)$ by $l_{1}=\ell-r_{p} \psi_{x}, l_{2}=\ell-r_{p} \psi_{y}, l_{3}=\ell+r_{p} \psi_{x}$, 
Chapter 3. Model predictive control of a robotically-actuated delivery sheath for beating heart compensation

and $l_{4}=\ell+r_{p} \psi_{y}$. Substituting the expressions for the tendon lengths $\left(l_{i}\right)$ into 3.3 and 3.4 yields

$$
\phi=\operatorname{atan} 2\left(\psi_{y}, \psi_{x}\right)
$$

and

$$
\kappa=\frac{r_{p} \sqrt{\psi_{x}^{2}+\psi_{y}^{2}}}{\ell d_{b}},
$$

respectively. Hence, we obtain the arc parameters $(\kappa, \phi$ and $\ell$ ) of the configurationspace as a function of the pulley angles $\left(\psi_{x}\right.$ and $\left.\psi_{y}\right)$ of the actuator-space.

The robot-independent mapping is given by the homogeneous transformation matrix $\left(\mathbf{H}_{i}^{0} \in S E 3\right)$, where

$$
\mathbf{H}_{i}^{0}=\left[\begin{array}{cccc}
c_{\phi} c_{\kappa \ell} & -s_{\phi} & c_{\phi} s_{\kappa \ell} & \frac{c_{\phi}\left(1-c_{\kappa \ell}\right)}{\kappa} \\
s_{\phi} c_{\kappa \ell} & c_{\phi} & s_{\phi} s_{\kappa \ell} & \frac{s_{\phi}\left(1-c_{\kappa \ell}\right)}{\kappa} \\
-s_{\kappa \ell} & 0 & c_{\kappa \ell} & \frac{s_{\kappa \ell}}{\kappa} \\
0 & 0 & 0 & 1
\end{array}\right],
$$

which describes the mapping between the intermediate frame $\left(\Psi_{i}\right)$ and the reference frame $\left(\Psi_{0}\right)$.

Remark (Discontinuity for $\kappa \neq 0$ ). Discontinuities in kinematics can be addressed by substituting differentiable cardinal sine and versine functions according to

$$
\operatorname{sinc}(\kappa \ell)=\left\{\begin{array}{ll}
\frac{\sin (\kappa \ell)}{\kappa \ell} & \kappa \ell \neq 0 \\
1 & \kappa \ell=0
\end{array},\right.
$$

and

$$
\operatorname{verc}(\kappa \ell)=\left\{\begin{array}{ll}
\frac{1-\cos (\kappa \ell)}{\kappa \ell} & \kappa \ell \neq 0 \\
0 & \kappa \ell=0
\end{array},\right.
$$

which are defined for $\kappa=0$.

In order to describe the articulating tip frame $\left(\Psi_{t}\right)$ of the RADS with respect to the intermediate frame $\left(\Psi_{i}\right)$ as demonstrated in Fig. 3.2 , we use a second transformation. This transformation describes the rigid link attached to the arc section (intermediate frame $\left(\Psi_{i}\right)$ ) of the RADS, which is given by homogenous transformation matrix $\left(\mathbf{H}_{t}^{i} \in S E 3\right)$, where

$$
\mathbf{H}_{t}^{i}=\left[\begin{array}{cc}
\mathbf{I}_{3} & \mathbf{L}_{t}^{i} \\
\mathbf{0}_{1 \times 3} & 1
\end{array}\right]
$$

where $\mathbf{I}$ and $\mathbf{0}$ represents an identity and a matrix filled with zeros, respectively. Further, $\mathbf{L}_{t}^{i} \in \mathbb{R}^{3}$, where $\mathbf{L}_{t}^{i}=\left[\begin{array}{lll}0 & 0 & l_{t}\end{array}\right]^{T}$ describes the rigid link section by 


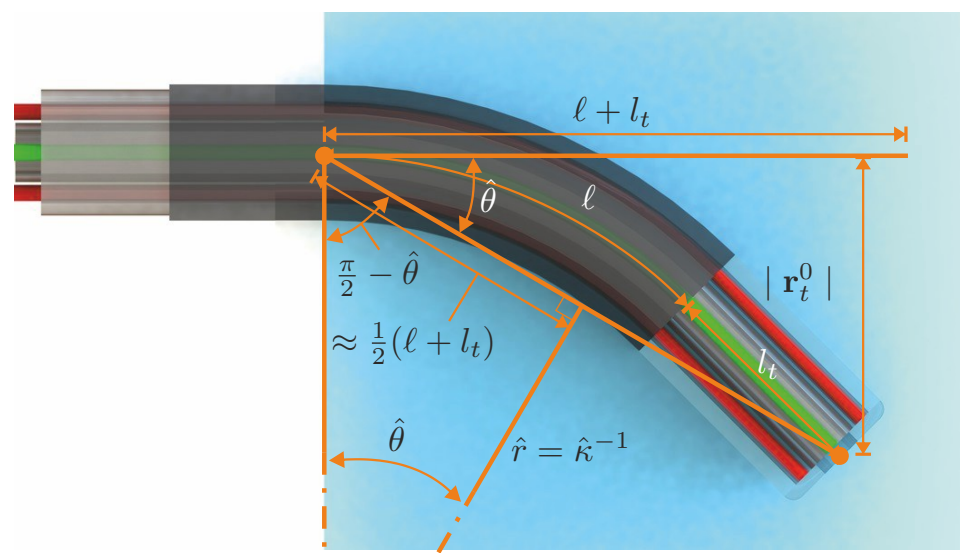

Figure 3.3: Overview of the geometric relations used to estimate an initial value for the arc curvature $(\hat{\kappa})$ of the robotically-actuated delivery sheath (RADS). The arc length $(\ell)$ and rigid link length $\left(l_{t}\right)$ combined with the RADS tip position $\left(\left|\mathbf{r}_{t}^{0}\right|\right)$ are used to estimate the bend angle $(\hat{\theta})$. The estimated bend angle $(\hat{\theta})$ and the approximated length $\left(\frac{1}{2}\left(\ell+l_{t}\right)\right)$ are used to evaluate an initial estimate of the arc curvature $(\hat{\kappa})$ of the RADS.

a translation along the $z$-axes of the intermediate frame $\left(\Psi_{i}\right)$. We describe the RADS articulating tip pose $\left(\mathbf{H}_{t}^{0}\right)$ in the reference frame $\left(\Psi_{0}\right)$ by a series of transformations according to $\mathbf{H}_{t}^{0}=\mathbf{H}_{i}^{0} \mathbf{H}_{t}^{i}$, which completes the forward kinematics.

\subsubsection{Inverse kinematics}

The inverse kinematics is used to express the pulley angles $\left(\psi_{x}\right.$ and $\left.\psi_{y}\right)$ as a function of the reference tip position $\left(\mathbf{r}_{t}^{0} \in \mathbb{R}^{3}\right.$, where $\left.\mathbf{r}_{t}^{0}=\left[r_{x} r_{y} r_{z}\right]^{T}\right)$ ). In order to evaluate the arc parameters of the configuration-space given a measured or reference tip position $\left(\mathbf{r}_{t}^{0}\right)$, we first determine the arc plane angle $(\phi)$ by

$$
\phi=\operatorname{atan} 2\left(r_{y}, r_{x}\right)
$$

Subsequently, we use the forward kinematics presented in (3.7) and 3.10 to derive an expression for the reference tip position $\left(\mathbf{r}_{t}^{0}\right)$ according to

$$
\left[\begin{array}{c}
\mathbf{r}_{t}^{0} \\
1
\end{array}\right]=\mathbf{H}_{t}^{0}\left[\begin{array}{c}
\mathbf{o}_{t} \\
1
\end{array}\right]=\left[\begin{array}{c}
c_{\phi}\left(\frac{\left(1-c_{\kappa \ell}\right)}{\kappa}+l_{t} s_{\kappa \ell}\right) \\
s_{\phi}\left(\frac{\left(1-c_{\kappa \ell}\right)}{\kappa}+l_{t} s_{\kappa \ell}\right) \\
\frac{s_{\kappa \ell}}{\kappa}+l_{t} c_{\kappa \ell} \\
1
\end{array}\right]
$$

where $\mathbf{o}_{t}=\left[\begin{array}{lll}0 & 0 & 0\end{array}\right]^{T} \in \mathbb{R}^{3}$ represents the origin of the articulating tip frame $\left(\Psi_{t}\right)$. Note, that the arc length $(\ell)$ and rigid link length $\left(l_{t}\right)$ are known. Thus, by 
Chapter 3. Model predictive control of a robotically-actuated delivery sheath for beating heart compensation

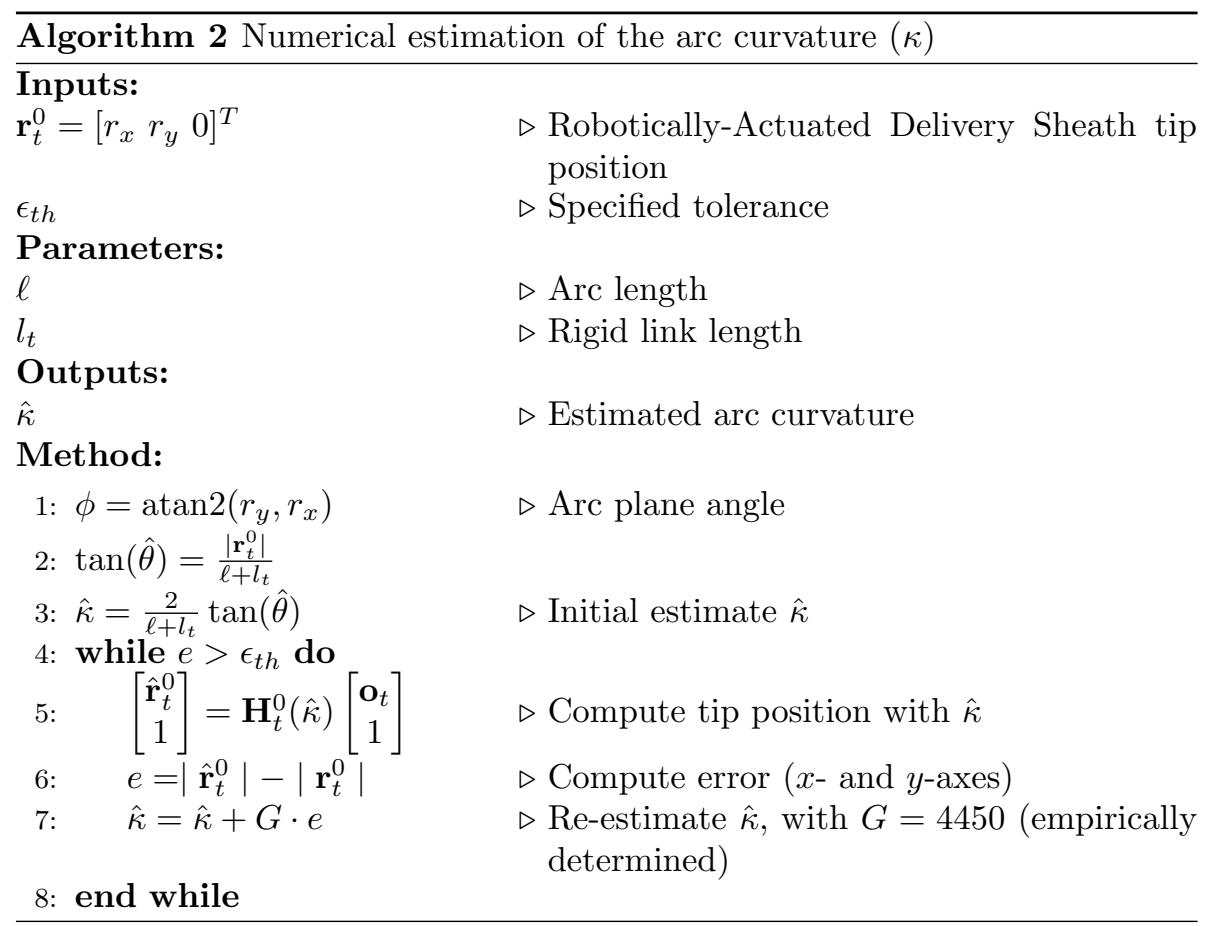

substituting (3.11) into 3.12 , the arc curvature $(\kappa)$ can be solved numerically as described in Algorithm 2. We first estimate an initial value for the arc curvature $(\hat{\kappa})$ using trigonometric relations as depicted in Fig. 3.3 . Subsequently, we use the initial estimated arc curvature $(\hat{\kappa})$ to compute the forward kinematics described in 3.7 and 3.10 . The error $(e)$ between the estimate $\left(\hat{\mathbf{r}}_{t}^{0}\right)$ and reference $\left(\mathbf{r}_{t}^{0}\right)$ tip position is evaluated. The computed error $(e)$ is used to correct the estimated arc curvature $(\hat{\kappa})$, where the feedback gain $(G)$ is included to obtain a desired rate of convergence. The process is repeated until the error $(e)$ is below a specified tolerance $\left(\epsilon_{t h}\right)$. Hence, we obtain an accurate estimation of the arc curvature $(\kappa)$. Given the evaluated arc curvature $(\kappa)$ and arc plane angle $(\phi)$, we can solve $(3.1)$ for all individual tendon lengths $\left(l_{i}\right)$ and determine the corresponding pulley angles $\left(\psi_{x}\right.$ and $\left.\psi_{y}\right)$, which completes the inverse kinematics.

\subsubsection{Differential kinematics}

Differential kinematics is used to relate the change in instrument arc parameters $\left(\mathbf{u}=\left[\begin{array}{ll}\phi & \kappa\end{array}\right]^{T}\right)$ to the change in tip position, which is integrated in the MPC strategy. Similar to the reference tip position in 3.12 , we can describe the tip position according to $\mathbf{p}=\mathbf{F}\left(\phi, \kappa, \ell, l_{t}\right)$, where $\mathbf{p}=\left[\begin{array}{lll}p_{x} & p_{y} & p_{z}\end{array}\right]^{T} \in \mathbb{R}^{3}$ is the tip position in $x$-, $y$ - and $z$-axes (frame $\left(\Psi_{0}\right)$ ), which is described by a 
function $\left(\mathbf{F}\left(\phi, \kappa, \ell, l_{t}\right)\right)$ of the arc parameters $(\phi$ and $\kappa)$. Given the instrument design, we consider the arc length $(\ell)$ and rigid link length $\left(l_{t}\right)$ to be constant in $\left(\mathbf{F}\left(\phi, \kappa, \ell, l_{t}\right)\right)$. Note, that the arc parameters $(\phi$ and $\kappa)$ are controlled by pulley angles $\left(\psi_{x}\right.$ and $\left.\psi_{y}\right)$. An expression that relates the change in instrument arc parameters $\left(\dot{\mathbf{u}}=\left[\begin{array}{ll}\dot{\phi} & \dot{\kappa}\end{array}\right]^{T}\right)$ to instrument tip velocity $\left(\dot{\mathbf{p}}=\left[\begin{array}{lll}\dot{p}_{x} & \dot{p}_{y} & \dot{p}_{z}\end{array}\right]^{T}\right)$ is given by

$$
\dot{\mathbf{p}}=\mathbf{J}_{R}(\mathbf{u}) \dot{\mathbf{u}}
$$

where

$$
\begin{aligned}
& \mathbf{J}_{R}(\mathbf{u})=\left[\begin{array}{cc}
\frac{\partial \mathbf{F}_{1}}{\partial \phi} & \frac{\partial \mathbf{F}_{1}}{\partial \kappa} \\
\frac{\partial \mathbf{F}_{2}}{\partial \phi} & \frac{\partial \mathbf{F}_{2}}{\partial \kappa} \\
\frac{\partial \mathbf{F}_{3}}{\partial \phi} & \frac{\partial \mathbf{F}_{3}}{\partial \kappa}
\end{array}\right]=
\end{aligned}
$$

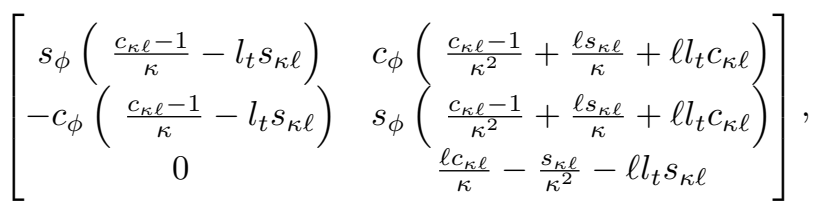

uses the forward kinematics described in 3.12 to analytically derive the differential kinematics. Note, that similar to (3.7), discontinuities for $\kappa=0$ can be addressed by using derivatives of cardinal sine $(\operatorname{sinc}(\kappa \ell))$ and versine $(\operatorname{verc}(\kappa \ell))$ functions. Furthermore, the kinematics of continuum-style robots using three actuators could be derived in a similar manner as presented by Simaan et al. 118. Given the presented techniques that provide forward, inverse and differential kinematics, often unknown modeling mismatches and external disturbances degrades the control performance. Hence, we include ultrasound feedback in order to improve our control strategy.

\subsubsection{Ultrasound image segmentation}

This section elaborates on the segmentation techniques applied to evaluate the RADS centroid location in 2D ultrasound images. In order to view the tip of the RADS in ultrasound images, we insert the instrument in a container and use water as an acoustic transport medium. A radial cross-sectional view of the RADS is obtained by orientating the 2D ultrasound image plane perpendicular to the shaft of the instrument (Fig. 3.4). The RADS tip is positioned in the ultrasound image plane by axial positioning, which is described in details in Section 3.2.3.4. Note, that by axial positioning, the segmented RADS tip frame in ultrasound images (frame $\left(\Psi_{u}\right)$ ) is expressed in the fixed reference frame $\left(\Psi_{0}\right)$ by position feedback.

A representative ultrasound image of the instrument tip is shown in Fig. 3.4(a). We observe a semi-circular shape that describes the reflecting surface of the RADS. By evaluation of the pre-operative ultrasound data (Fig. 3.6), we did 
Chapter 3. Model predictive control of a robotically-actuated delivery sheath for beating heart compensation

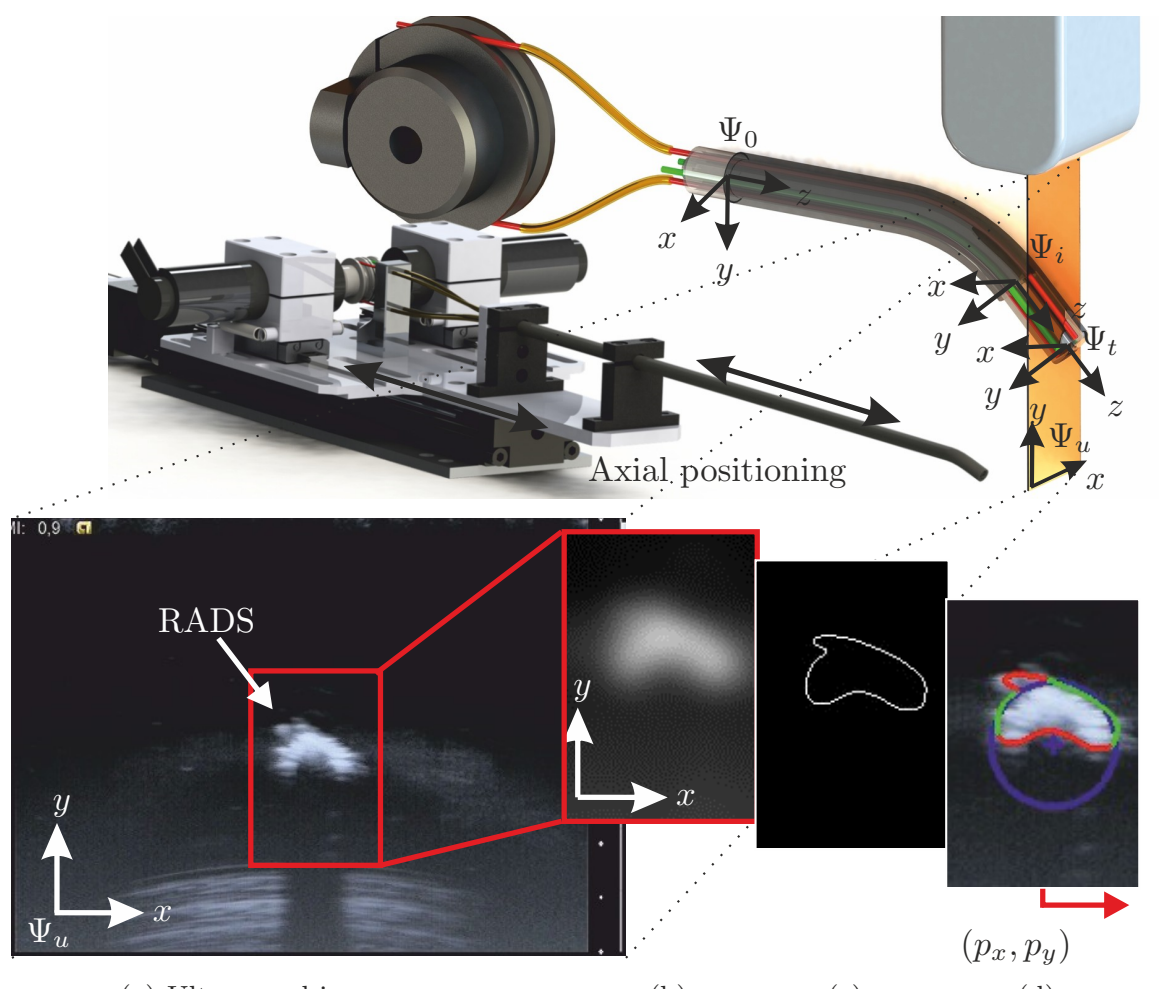

(a) Ultrasound image

(b)

(c)

(d)

Figure 3.4: Ultrasound image segmentation to evaluate the centroid location $\left(p_{x}, p_{y}\right)$ of the robotically-actuated delivery sheath (RADS). The RADS tip (frame $\left(\Psi_{t}\right)$ ) is positioned in the ultrasound image plane denoted by frame $\left(\Psi_{u}\right)$ using axial positioning along the $x$ axis (frame $\left(\Psi_{0}\right)$ ). (a) Radial cross-sectional view of the RADS in 2D ultrasound images. (b) Gaussian filtering using a two-dimensional kernel. (c) Canny edge detection with hysteresis thresholding. (d) Random sample consensus (RANSAC) to localize the centroid $\left(p_{x}, p_{y}\right)$ of the RADS (center of the blue circle). The green and red points are considered inliers and outliers, respectively.

not observe identical semi-circular shapes that represented anatomical structures. Therefore, we use segmentation techniques based on the circular shape parameters to localize the tip of the RADS in ultrasound images. Further, the acoustic impedance difference between RADS materials and cardiac tissue is considered to be significantly large, which enhances the visibility of the instrument 95, 111]. This would enable instrument detection when interaction with cardiac tissue is considered.

Preprocessing is performed to enhance the visibility of the RADS in ultrasound images. In order to reduce speckle and to smoothen edges in the ultrasound image, a 2D Gaussian kernel is applied, as depicted in Fig. 3.4(b). The contrast between instrument and the environment in ultrasound images is 
sufficient for edge detection. Hence, we use a Canny edge detector with hysteresis thresholding to obtain an edge map of the ultrasound image as shown in Fig. 3.4 (c) 119. Hysteresis thresholding reduces the detection of irrelevant edges, that do not describe the semi-circular surface of the RADS. However, irrelevant edges, often introduced by surface deformations caused by artifacts and bending of the instrument may still exist. Hence, the centroid location is evaluated using a random sample consensus (RANSAC) strategy (Algorithm 3). RANSAC is robust to irrelevant edges that do not fit the parametric description of the semi-circular model representing the RADS [119].

The evaluated set $\left(A_{c}\right)$ of edge points $\left(\mathbf{x}_{c} \in \mathbb{R}^{2}\right)$ from the Canny edge detector is used as an input to the RANSAC algorithm. The RANSAC algorithm is an iterative process that consists of three steps (i.e., (I) Hypothesis, (II) Pre-

$\overline{\text { Algorithm } 3 \text { Random sample consensus robotically-actuated delivery sheath }}$ localization

\section{Inputs:}

$A_{c} \leftarrow\left\{\mathbf{x}_{c, v \mid v=1, \cdots, w}\right\}$

$f: H_{3} \rightarrow m_{c}$

$\triangleright$ Set of detected edge points $\left(\mathbf{x}_{c}\right)$

$\triangleright$ Computes the algebraic circle model parameters $\left(m_{c}\right)$ from a set $\left(H_{3}\right)$ of three randomly selected edge points

$C\left(m_{c}, \mathbf{x}_{c}\right)$

$\triangleright$ Cost-function for a single edge point (1 if $\mathbf{x}_{c}$ is an inlier to the algebraic circle parameters $\left(m_{c}\right), 0$ otherwise)

$n$

$\triangleright$ Number of iterations

\section{Outputs:}

$m_{c}^{*}$

$S_{c}^{*}$

$J_{c}^{*}$

\section{Method:}

1: for $i \leftarrow 1, n$ do

2: $\quad H_{3, i} \leftarrow$ random_3pnts $\left(A_{c}\right)$

3: $\quad m_{c}, i \leftarrow f\left(H_{3, i}\right)$

4: if suffice $\left(m_{c, i}\right)$ then

5: $\quad S_{c}, i \leftarrow\left\{\forall \mathbf{x}_{c} \in A_{c} \mid C\left(m_{c, i}, \mathbf{x}_{c}\right)=1\right\}$

6: $\quad J_{c}, i \leftarrow \sum_{\mathbf{x}_{c} \in A_{c}} C\left(m_{c, i}, \mathbf{x}_{c}\right)$

7: $\quad$ if $J_{c}^{*}<J_{c}, i$ then

8: $\quad J_{c}^{*} \leftarrow J_{c, i}$

9: $\quad m_{c}^{*} \leftarrow m_{c, i}$

10: $\quad S_{c}^{*} \leftarrow S_{c, i}$

11: end if

12: $\quad$ end if

13: $\quad i \leftarrow i+1$

14: end for $\triangleright$ Best model parameters

$\triangleright$ Best consensus set (inliers)

$\triangleright$ Best cost

rar 
Chapter 3. Model predictive control of a robotically-actuated delivery sheath for beating heart compensation

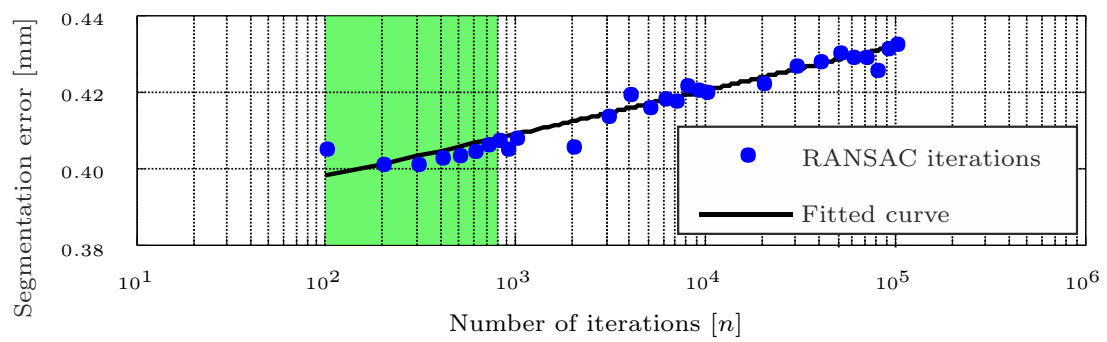

(a) RANSAC iterations vs Manual segmentation

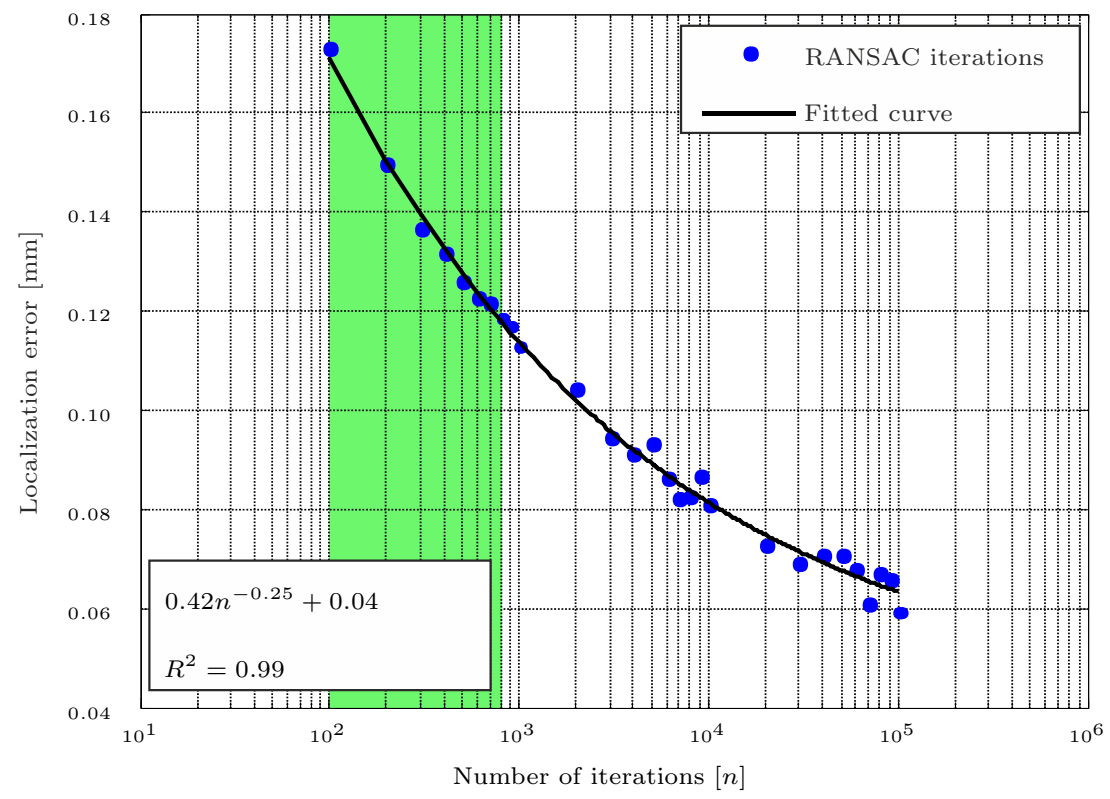

(b) RANSAC iterations vs Centroid localization error

Figure 3.5: Experimental evaluation and validation of image segmentation using a sequence of 600 ultrasound images. (a) Compares the performance between varying random sample consensus (RANSAC) iterations using a ground truth obtained by manual segmentation. (b) Describes the relation between the number of iterations and the localization error using a ground truth obtained after $10^{6}$ RANSAC iterations.

liminary test and (III) Evaluation). In $(I)$, a hypothetical solution is found by fitting algebraic circle model parameters $(m)$ for $f: H_{3} \rightarrow m_{c}$ to a set $\left(H_{3}\right)$ of candidate inliers. The set $\left(H_{3}\right)$ consists of three arbitrary selected edge points from the evaluated set $\left(A_{c}\right)$. In $(I I)$, a preliminary test $\left(\right.$ suffice $\left.\left(m_{c, i}\right)\right)$ is performed to evaluate the model parameters. The model parameters such as radius and centroid location should be consistent with those of the RADS in order to qualify as a potential solution. Further, all edge points of the edge 
map are evaluated using a cost-function $\left(C\left(m_{c}, \mathbf{x}_{c}\right)\right)$ to determine if they are sufficiently close to the periphery of the circular shape. The fitted model is acceptable if a sufficiently large part of the semi-circular surface has been evaluated as the consensus set. In $(I I I)$, an evaluation is performed in order to improve the previous solution. The model parameters and consensus set are both refined if the computed cost $\left(J_{c, i}\right)$ of the current iteration exceeds the previous solution. After $n$ iterations, the centroid location $\left(p_{x}, p_{y}\right)$ of RADS is evaluated from the model parameters $\left(m_{c}\right)$ and displayed as the center of the circle (Fig. 3.4(d)).

The performance of the RANSAC algorithm is evaluated and validated experimentally using a sequence of 600 ultrasound images. Experiments show that the localization error of the RADS decreases if the number $(n)$ of iterations of the RANSAC algorithm are increased as depicted in Fig. 3.5(b). In order to minimize computational costs and considering the ultrasound imaging resolution of approximately $0.12 \mathrm{~mm}$ per pixel, the number of RANSAC iterations should be limited to approximately 800, which is depicted green in Fig. 3.5. Further, we compare the results of RADS segmentation for varying RANSAC iterations using manual segmentation as a ground truth (Fig. 3.5(a)). The results show, that by increasing the RANSAC iterations, the segmentation error remains approximately constant $(0.4 \mathrm{~mm})$. Since there is no reason to believe that an increase in RANSAC iterations would deteriorate segmentation accuracy, we estimate that the insignificant increased error in Fig. 3.5.a), could be attributed to imperfections in manual segmentations.

The presented ultrasound segmentation method is used in our closed-loop control strategy to improve the performance. Note, that the presented 2D segmentation method can be expanded to 3D ultrasound imaging by parallel evaluation of slices along the instrument shaft.

\subsubsection{Model predictive control}

In this section we present the Model Predictive Control (MPC) architecture used to control the RADS in closed-loop. The objective of the MPC architecture is to anticipate the AHV motion based on a model. This allows the clinician to focus on the primary task of the procedure, while compensation for AHV motions is provided. In order to develop an AHV model, we use the pre-operative $2 \mathrm{D}$ ultrasound images that describes the AHV motion of a human during the cardiac cycle. Further, we integrate forward, inverse and differential kinematics described in Section 3.2.1 to determine instrument motion. The 2D ultrasound segmentation presented in Section 3.2 .2 is used to provide instrument feedback for control. 
Chapter 3. Model predictive control of a robotically-actuated delivery sheath for beating heart compensation

\subsubsection{Model description}

The continuous time representation of the model used in the MPC strategy incorporates the differential kinematics described in 3.13 . A cascade configuration is used according to

$$
\begin{gathered}
\dot{\mathbf{p}}_{(t)}=\mathbf{J}_{R}\left(\mathbf{u}_{(t)}\right) \dot{\mathbf{u}}_{(t)}, \\
\ddot{\mathbf{u}}_{(t)}=\mathbf{v}_{(t)}+\mathbf{e}_{(t)}, \\
\mathbf{y}_{(t)}=\mathbf{p}_{(t)}+\mathbf{e}_{(t)},
\end{gathered}
$$

where $\mathbf{v}_{(t)}$ is considered to be the MPC control input signal. However, the change in arc parameters $\left(\dot{\mathbf{u}}_{(t)}\right)$ is used to steer the RADS according to the MPC strategy. The system is subject to an inequality constraint according to

$$
\mathbf{p}_{\min } \leq \mathbf{p}_{(t)} \leq \mathbf{p}_{\max } \forall t
$$

where the instrument tip position is restricted. The instrument tip position is limited in order to prevent damage to the instrument arc-section. Further, by adding restricted regions for the instrument tip position, damage to sensitive tissue can be avoided. In order to preserve dominant features required for ultrasound image segmentation, we add an additional inequality constraint. The constraint is used to limit the instrument tip velocity in $x$ - and $y$-directions of frame $\left(\Psi_{0}\right)$ by

$$
\dot{\mathbf{p}}_{\text {min }} \leq \dot{\mathbf{p}}_{(t)} \leq \dot{\mathbf{p}}_{\max } \forall t
$$

The discrete time model integrated within the MPC strategy can be obtained by using forward Euler discretization of the continuous time model according to

$$
\begin{gathered}
\mathbf{p}_{(k+1)}=\mathbf{p}_{(k)}+\mathbf{J}_{R}\left(\mathbf{u}_{(k)}\right) \mathbf{a}_{(k)}, \\
\mathbf{a}_{(k+1)}=\mathbf{a}_{(k)}+T_{s}^{2} \mathbf{v}_{(k)}+T_{s}^{2} \mathbf{e}_{(k)}, \\
\mathbf{y}_{(k)}=\mathbf{p}_{(k)}+\mathbf{e}_{(k)},
\end{gathered}
$$

where we substitute $\mathbf{a}_{(k)}=\mathbf{u}_{(k+1)}-\mathbf{u}_{(k)}$ and $T_{s}$ denotes the sampling time. Similarly, the equivalent discrete time constraints are given by

$$
\mathbf{p}_{\min } \leq \mathbf{p}_{(k)} \leq \mathbf{p}_{\max } \forall k,
$$

and

$$
\dot{\mathbf{p}}_{\text {min }} \leq \frac{\mathbf{p}_{(k+1)}-\mathbf{p}_{(k)}}{T_{s}} \leq \dot{\mathbf{p}}_{\max } \forall k .
$$

Note, that the constraints stacked into a vector can be evaluated componentwise. The presented discrete time model is integrated within MPC strategy to optimize the control objective. 


\subsubsection{Control objective}

The main MPC objective is to anticipate the AHV motion, while considering a desired reference input. In order to capture these requirements, we can formulate a reference tracking objective according to

$$
\mathbf{r}=\mathbf{r}_{A H V}+\mathbf{r}_{d}
$$

where $\mathbf{r}_{d}$ describes the RADS desired tip position without a priori knowledge, while $\mathbf{r}_{A H V}$ represents the position of the AHV with a priori knowledge obtained from pre-operative modeling. In this study, we assume that a priori knowledge on AHV position is sufficiently well-described by modeling, which is presented in Section 3.2.3.3. In order to achieve our control objective in MPC, we formulate a generalized predictive control (GPC) cost-function $(J)$ [120]. This cost-function evaluates, over a horizon, the reference tracking error and the control action by

$$
\begin{array}{r}
J_{(\mathbf{v}, k)}=\sum_{j=N_{m}}^{N}\left(\hat{\mathbf{y}}_{(k+j \mid k)}-\mathbf{r}_{(k+j)}\right)^{T}\left(\hat{\mathbf{y}}_{(k+j \mid k)}-\mathbf{r}_{(k+j)}\right) \\
+\sum_{j=1}^{N} \mathbf{v}_{(k+j-1 \mid k)}^{T} \boldsymbol{\lambda}^{T} \boldsymbol{\lambda} \mathbf{v}_{(k+j-1 \mid k)},
\end{array}
$$

where $N=15$ describes the prediction horizon, $N_{m}$ is the minimum costhorizon and equals one, $\left(\hat{\mathbf{y}}_{(k+j \mid k)}\right)$ is the predicted instrument tip position $\left(\mathbf{y}_{(k+j)}\right)$ based on knowledge up to time $(k)$ and $\boldsymbol{\lambda}$ is the control input weighting matrix given by

$$
\boldsymbol{\lambda}=\left[\begin{array}{cc}
1 \times 10^{-2} & 0 \\
0 & 1 \times 10^{-7}
\end{array}\right]
$$

which evaluates the control action $(\mathbf{v})$. The control input weighting matrix $(\boldsymbol{\lambda})$, the prediction horizon $(N)$ and minimum cost-horizon $\left(N_{m}\right)$ are empirically determined. By rewriting the cost-function 3.26 into

$$
J_{(\mathbf{v}, k)}=\sum_{j=0}^{N-1} \hat{\mathbf{z}}_{(k+j \mid k)}^{T} \boldsymbol{\Gamma}_{(j)} \hat{\mathbf{z}}_{(k+j \mid k)},
$$

we obtain the standard form used in MPC, where

$$
\mathbf{z}_{(k)}=\left[\begin{array}{c}
\hat{\mathbf{y}}_{(k+1 \mid k)}-\mathbf{r}_{(k+1)} \\
\boldsymbol{\lambda} \mathbf{v}_{(k)}
\end{array}\right],
$$

describes the reference tracking error and control action, while

$$
\boldsymbol{\Gamma}_{(j)}= \begin{cases}{\left[\begin{array}{cc}
\mathbf{0}_{3 \times 3} & \mathbf{0}_{3 \times 2} \\
\mathbf{0}_{2 \times 3} & \mathbf{I}_{2}
\end{array}\right] \quad \text { for } 0 \leq j<N_{m}-1} \\
{\left[\begin{array}{cc}
\mathbf{I}_{3} & \mathbf{0}_{3 \times 2} \\
\mathbf{0}_{2 \times 3} & \mathbf{I}_{2}
\end{array}\right] \quad \text { for } N_{m}-1 \leq j<N-1}\end{cases}
$$


Chapter 3. Model predictive control of a robotically-actuated delivery sheath for beating heart compensation

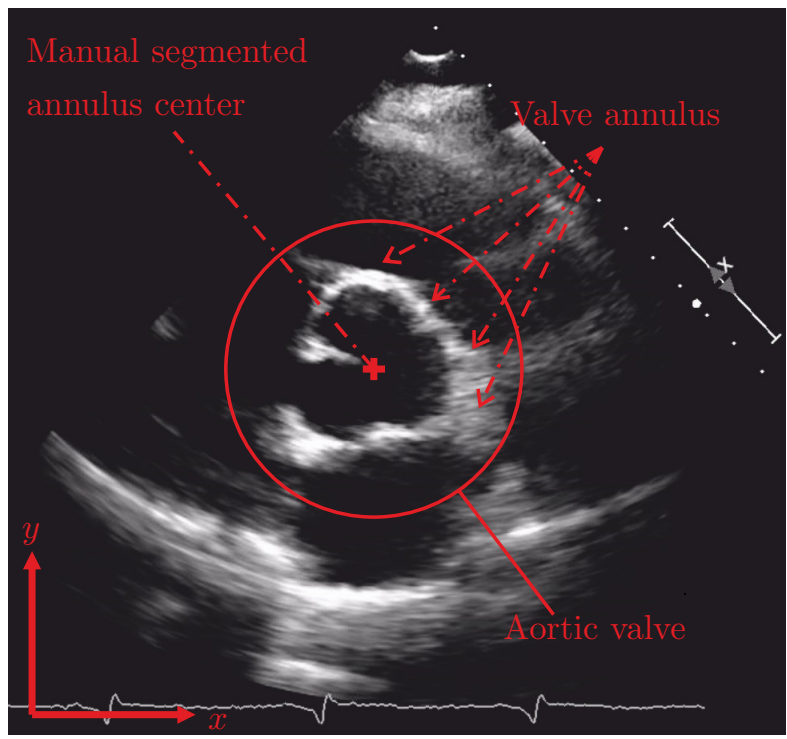

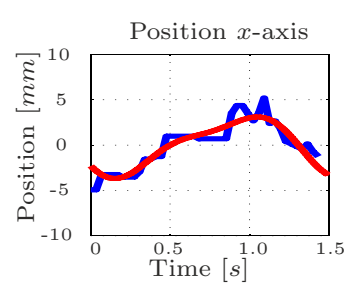

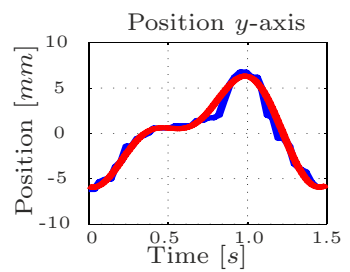

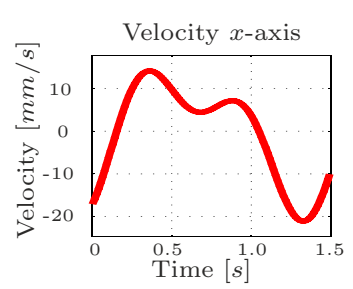

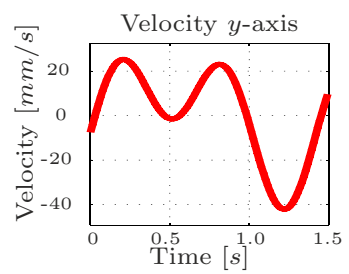

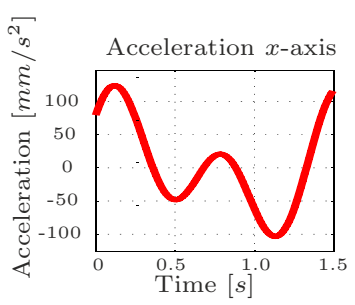

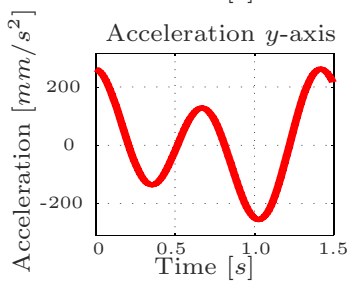

Figure 3.6: A pre-operative two-dimensional transesophageal echocardiogram (TEE) of the aortic heart valve (AHV) annular plane. The center location of the aortic heart valve annulus is manually segmented for multiple cardiac cycles. The human evaluated annulus center positions are used for fitting a motion model. The blue line demonstrates the manuallysegmented annulus position during the cardiac cycle. The corresponding red line describes the position of the fitted model. Further, the velocities and accelerations of the AHV models are provided.

is a diagonal selection matrix $\left(\boldsymbol{\Gamma}_{(j)} \in \mathbb{R}^{5 \times 5}\right)$ used to describe the horizon of the cost-function. By minimizing the cost-function $\left(J_{(\mathbf{v}, k)}\right)$, we optimize between RADS tracking accuracy and control effort, where the reference $\left(\mathbf{r}_{A H V}\right)$ is given by AHV modeling. 


\subsubsection{Aortic heart valve modeling}

Modeling is used to anticipate the AHV motion in MPC. As a case study, we observe the motion of the AHV annulus during the human cardiac cycle. We use pre-operative 2D transesophageal echocardiogram (TEE) to obtain the motion profile of a human AHV annulus (Fig. 3.6). The motion profile obtained from 2D ultrasound images is used to demonstrate compensation of the AHV motion. In this study, we assume a constant shape of the aortic annulus. Hence, we obtain the center position of the aortic annulus by manual segmentation (Fig. 3.6).

The manual segmented aortic annulus position during the cardiac cycle is used to derive a model capable of describing the periodic motion. A two-term Fourier series according to

$$
f_{x}=a_{0_{x}}+a_{1_{x}} c_{\frac{k}{S_{r}} \omega}+b_{1_{x}} s_{\frac{k}{S_{r}} \omega}+a_{2_{x}} c_{2 \frac{k}{S_{r}} \omega}+b_{2 x} s_{2 \frac{k}{S_{r}} \omega},
$$

and

$$
f_{y}=a_{0_{y}}+a_{1 y} c_{\frac{k}{S_{r}} \omega}+b_{1_{y}} s_{\frac{k}{S_{r}} \omega}+a_{2 y} c_{2 \frac{k}{S_{r}} \omega}+b_{2 y} s_{2 \frac{k}{S_{r}} \omega},
$$

is used to describe the periodic aortic annulus motion, where $k$ describes discrete time, $S_{r}$ denotes the number of samples per second and $\omega$ represents the frequency of the periodic function in radians per second. The frequency $(\omega)$ is given by $\omega=\frac{\omega_{x}+\omega_{y}}{2}$, and relates to the heart rate $\left(H_{r}\right)$ in beats-per-minute (BPM) according to $H_{r}=\frac{60 \omega}{2 \pi}$. The corresponding coefficients and frequencies $\left(a_{0_{*}}, a_{1_{*}}, b_{1_{*}}, a_{2_{*}}, b_{2_{*}}\right.$ and $\left.\omega_{*}\right)$ are provided in Table 3.1. By considering the frequencies provided in Table 3.1. the observed heart rate $\left(H_{r}\right)$ is 42 BPM. The goodness of data fitting is given by the coefficients of determination $R^{2}=0.86$ and $R^{2}=0.97$ along the the $x$ - and $y$-axes, respectively. Note, that an increase in the number of terms of the Fourier series described in 3.31 and 3.32 does not result in a significant improvement in the coefficients of determination. From the manual segmentation we observe displacements of $9.60 \pm 3.23 \mathrm{~mm}$ in $x$-axis, while the $y$-axis shows displacements of $12.25 \pm 1.27 \mathrm{~mm}$. Further, from the fitted model we observe maximum velocities and accelerations of approximately $40 \mathrm{~mm} / \mathrm{s}$ and $250 \mathrm{~mm} / \mathrm{s}^{2}$, respectively. The periodic aortic annulus motions described by $f_{x}$ and $f_{y}$ provides the tracking reference $\left(\mathbf{r}_{A H V}=\left[\begin{array}{lll}f_{x} & f_{y} & 0\end{array}\right]^{T}\right)$ for MPC. In order to anticipate the motion of the aortic annulus, a priori knowledge of the tracking reference $\left(\mathbf{r}_{A H V}\right)$ is considered during experimental evaluation using MPC.

Table 3.1: Table of Fourier coefficients used in 3.31 and 3.32 to describe the periodic aortic heart valve motion depicted in Fig. 3.6 Subscript * denotes the corresponding $x$ - or $y$-axes

\begin{tabular}{lllllll}
\hline & $a_{0_{*}}$ & $a_{1_{*}}$ & $b_{1_{*}}$ & $a_{2_{*}}$ & $b_{2_{*}}$ & $\omega_{*}$ \\
\hline$x$-axis & 0.0547 & -1.7850 & -2.4530 & -0.5737 & -0.7064 & 4.4050 \\
\hline$y$-axis & 0.3157 & -3.7290 & -2.7090 & -2.4190 & 0.4965 & 4.4240 \\
\hline
\end{tabular}


Chapter 3. Model predictive control of a robotically-actuated delivery sheath for beating heart compensation

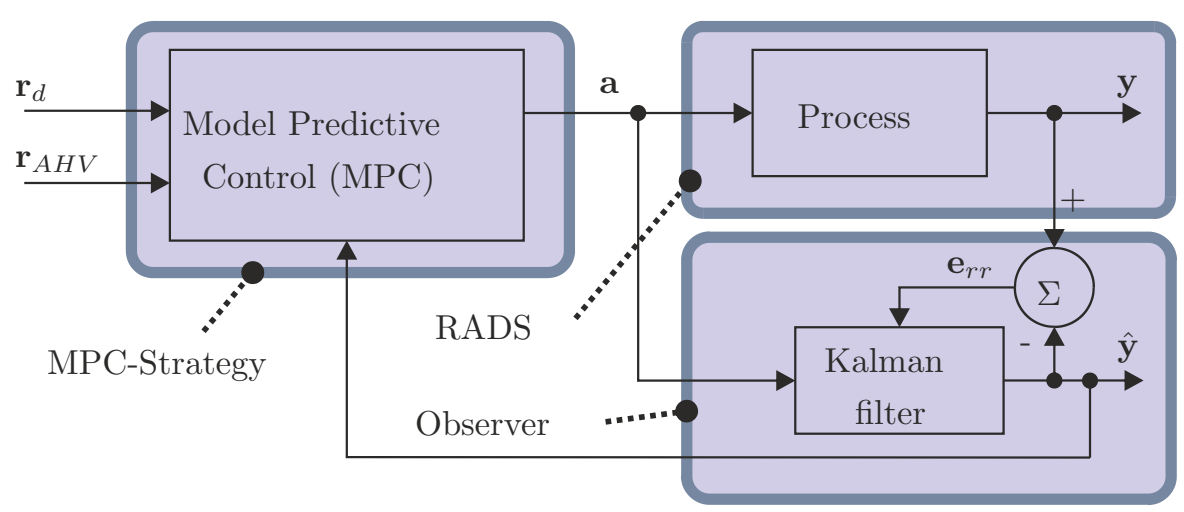

Figure 3.7: Model predictive control (MPC) strategy is used to steer the robotically-actuated delivery sheath (RADS). The referenced tip position by the clinician is denoted by $\mathbf{r}_{d}$, while $\mathbf{r}_{A H V}$ describes the position of the aortic heart valve with a priori knowledge obtained from pre-operative ultrasound images. The tip position obtained by ultrasound image segmentation is denoted by $\mathbf{y}$, with corresponding filtered position described by $\hat{\mathbf{y}}$. The arc parameters denoted by $\mathbf{a}$ is provided as an input to the RADS and the Kalman filter. The resulting positioning error is given by $\mathbf{e}_{r r}$, which is used to adapt the Kalman filter.

\subsubsection{Controller design}

The MPC strategy used to control the articulating tip of the RADS is presented in Fig. 3.7. The control variable ( $\mathbf{v})$ is used to determine the change in arc parameters (a), which are implemented in order to position the tip of the RADS. Subsequently, the tip position $\left(\mathbf{y}=\left[\begin{array}{lll}p_{x} & p_{y} & 0\end{array}\right]^{T}\right)$ of the RADS is measured using a $2 \mathrm{D}$ ultrasound transducer as described in Section 3.2.2. However, ultrasound images are often prone to noise and the tip of the RADS may not always be detected during tracking. Hence, we add an extended Kalman filter to provide state estimation $\left(\hat{\mathbf{y}}=\left[\begin{array}{lll}\hat{p}_{x} & \hat{p}_{y} & \hat{p}_{z}\end{array}\right]^{T}\right)$ based on kinematics described in Section 3.2.1 and 2D ultrasound measurements 97. The position error $\left(\mathbf{e}_{r r}=\mathbf{y}-\hat{\mathbf{y}}\right)$ is evaluated in order to adapt the Kalman filter. Note, that the articulating tip of the RADS must intersect with the ultrasound image plane in order to provide position feedback. Hence, we limit the articulating tip motion to the ultrasound image plane by autonomous axial positioning of the RADS along the $z$-axis of frame $\left(\Psi_{0}\right)$ using a linear stage. The state estimate $\left(\hat{p}_{z}\right)$ provided by the Kalman filter is used as an input to axial positioning.

In order to describe the MPC strategy, we formulate a constrained standard predictive control problem (CSPCP), which uses a multiple-input and a multiple-output (MIMO) state-space representation of the system [120,121. We can write the CSPCP using a MIMO model of the RADS in state-space realization according to the following:

$$
\mathbf{x}_{(k+1)}=\mathbf{A} \mathbf{x}_{(k)}+\mathbf{B}_{1} \mathbf{e}_{(k)}+\mathbf{B}_{2} \mathbf{w}_{(k)}+\mathbf{B}_{3} \mathbf{v}_{(k)},
$$




$$
\begin{gathered}
\mathbf{y}_{(k)}=\mathbf{C}_{1} \mathbf{x}_{(k)}+\mathbf{D}_{11} \mathbf{e}_{(k)}+\mathbf{D}_{12} \mathbf{w}_{(k)}+\mathbf{D}_{13} \mathbf{v}_{(k)}, \\
\mathbf{z}_{(k)}=\mathbf{C}_{2} \mathbf{x}_{(k)}+\mathbf{D}_{21} \mathbf{e}_{(k)}+\mathbf{D}_{22} \mathbf{w}_{(k)}+\mathbf{D}_{23} \mathbf{v}_{(k)}, \\
\boldsymbol{\chi}_{(k)}=\mathbf{C}_{4} \mathbf{x}_{(k)}+\mathbf{D}_{41} \mathbf{e}_{(k)}+\mathbf{D}_{42} \mathbf{w}_{(k)}+\mathbf{D}_{43} \mathbf{v}_{(k)} \leq \boldsymbol{X}_{(k)},
\end{gathered}
$$

where we use the model described in 3.20 to 3.22 . The state is given by $\mathbf{x}_{(k)}=\left[\begin{array}{ll}\mathbf{p}_{(k)} & \mathbf{a}_{(k)}\end{array}\right]^{T}, \mathbf{e}_{(k)}$ represents zero mean white noise, while $\mathbf{w}_{(k)}=$ $\left[\begin{array}{ll}\mathbf{d}_{(k)} & \mathbf{r}_{(k+1)}\end{array}\right]^{T}$ combines all known external signals such as disturbance $\left(\mathbf{d}_{(k)}\right)$ and reference $\left(\mathbf{r}_{(k+1)}\right.$, as described in $\left.(3.25)\right)$. Further, the generalized input is described by $\mathbf{v}_{(k)}$. Note, that $\mathbf{y}_{(k)}$ considers all past inputs and all previous and current measurements $\mathbf{y}_{(0)}, \ldots, \mathbf{y}_{(k)}$, which are provided by segmentation of the RADS tip in ultrasound images. The state-space matrices in (3.33) and (3.34) are given by

$$
\begin{array}{r}
\mathbf{A}=\left[\begin{array}{cc}
\mathbf{I}_{3} & \mathbf{J}_{R}(\mathbf{u}) \\
\mathbf{0}_{2 \times 3} & \mathbf{I}_{2}
\end{array}\right], \mathbf{B}_{1}=\left[\begin{array}{cc}
\mathbf{0}_{3 \times 3} & \mathbf{0}_{3 \times 2} \\
\mathbf{0}_{2 \times 3} & T_{s}^{2} \mathbf{I}_{2}
\end{array}\right], \\
\mathbf{B}_{2}=\left[\begin{array}{cc}
T_{s} \mathbf{I}_{3} & \mathbf{0}_{3 \times 3} \\
\mathbf{0}_{2 \times 3} & \mathbf{0}_{2 \times 3}
\end{array}\right], \mathbf{B}_{3}=\left[\begin{array}{l}
\mathbf{0}_{3 \times 2} \\
T_{s}^{2} \mathbf{I}_{2}
\end{array}\right], \mathbf{C}_{1}=\left[\begin{array}{ll}
\mathbf{C}_{0} & \mathbf{0}_{3 \times 2}
\end{array}\right], \\
\mathbf{D}_{11}=\left[\mathbf{I}_{2} \mathbf{0}_{3 \times 2}\right], \mathbf{D}_{12}=\mathbf{0}_{3 \times 6}, \mathbf{D}_{13}=\mathbf{0}_{3 \times 2},
\end{array}
$$

where we use the state $(\mathbf{p})$ and the inverse kinematics described in Section 3.2.1.2 to provide the arc parameters $(\mathbf{u})$ for the differential kinematics incorporated in matrix (A). Further, the output of the system is limited to the $2 \mathrm{D}$ ultrasound image plane, which is given in $x$ - and $y$ directions of frame $\left(\Psi_{0}\right)$ by

$$
\mathbf{C}_{0}=\left[\begin{array}{lll}
1 & 0 & 0 \\
0 & 1 & 0 \\
0 & 0 & 0
\end{array}\right]
$$

In this study, we do not model external disturbances, hence we denote $\mathbf{d}_{(k)}=$ $\left[\begin{array}{lll}0 & 0 & 0\end{array}\right]^{T}, \forall k$. The matrices $\mathbf{C}_{2}, \mathbf{D}_{21}, \mathbf{D}_{22}$ and $\mathbf{D}_{23}$ associated with the cost signal $\left(\mathbf{z}_{(k)}\right)$ introduced in 3.35 can be obtained by substituting $\hat{\mathbf{y}}_{(k+1 \mid k)}=$ $\mathbf{C}_{1} \mathbf{x}_{(k+1)}+\mathbf{D}_{11} \hat{\mathbf{e}}_{(k+1 \mid k)}$ and $\mathbf{v}_{(k)}$ into 3.29 , which can be rewritten as

$$
\begin{array}{r}
\hat{\mathbf{z}}_{(k)}=\underbrace{\left[\begin{array}{c}
\mathbf{C}_{1} \mathbf{A} \\
\mathbf{0}_{2 \times 6}
\end{array}\right]}_{\mathbf{C}_{2}} \mathbf{x}_{(k)}+\underbrace{\left[\begin{array}{c}
\mathbf{C}_{1} \mathbf{B}_{1} \\
\mathbf{0}_{2 \times 3}
\end{array}\right]}_{\mathbf{D}_{21}} \mathbf{e}_{(k)} \\
+\underbrace{\left(\left[\begin{array}{c}
\mathbf{C}_{1} \mathbf{B}_{2} \\
\mathbf{0}_{2 \times 6}
\end{array}\right]+\left[\begin{array}{cc}
\mathbf{0}_{3 \times 3} & -\mathbf{I}_{3 \times 3} \\
\mathbf{0}_{2 \times 3} & \mathbf{0}_{2 \times 3}
\end{array}\right]\right)}_{\mathbf{D}_{22}} \mathbf{w}_{(k)}+\underbrace{\left[\begin{array}{c}
\mathbf{C}_{1} \mathbf{B}_{3} \\
\boldsymbol{\lambda}
\end{array}\right]}_{\mathbf{D}_{23}} \mathbf{v}_{(k)},
\end{array}
$$


Chapter 3. Model predictive control of a robotically-actuated delivery sheath for beating heart compensation

where we use the zero mean white noise estimate $\left(\hat{\mathbf{e}}_{(k+1 \mid k)}=\mathbf{0}_{3 \times 1}\right)$.

The constraints presented in $(3.23)$ and $(3.24)$ can be combined and described by two one-sided constraint signals $\left(\chi_{1,(k)}\right.$ and $\left.\chi_{2,(k)}\right)$ stacked together according to

$$
\begin{array}{r}
\underbrace{\left[\begin{array}{c}
\boldsymbol{\chi}_{1,(k)} \\
\boldsymbol{\chi}_{2,(k)}
\end{array}\right]}_{\boldsymbol{\chi}_{(k)}}=\left[\begin{array}{c}
\mathbf{x}_{(k+1)} \\
-\mathbf{x}_{(k+1)}
\end{array}\right]=\underbrace{\left[\begin{array}{c}
\mathbf{A} \\
-\mathbf{A}
\end{array}\right]}_{\mathbf{C}_{4}} \mathbf{x}_{(k)}+\underbrace{\left[\begin{array}{c}
\mathbf{B}_{1} \\
-\mathbf{B}_{1}
\end{array}\right]}_{\mathbf{D}_{41}} \mathbf{e}_{(k)}+ \\
\underbrace{\left[\begin{array}{c}
\mathbf{B}_{2} \\
-\mathbf{B}_{2}
\end{array}\right]}_{\mathbf{D}_{42}} \mathbf{w}_{(k)}+\underbrace{\left[\begin{array}{c}
\mathbf{B}_{3} \\
-\mathbf{B}_{3}
\end{array}\right]}_{\mathbf{D}_{43}} \mathbf{v}_{(k)} \leq \underbrace{\left[\begin{array}{c}
\boldsymbol{X}_{1, \max } \\
-\boldsymbol{X}_{2, \min }
\end{array}\right]}_{\boldsymbol{X}_{(k)}},
\end{array}
$$

where $\boldsymbol{X}_{1, \max }=\left[\begin{array}{ll}\mathbf{p}_{\max } & T_{s} \mathbf{J}_{R}^{+}(\mathbf{u}) \dot{\mathbf{p}}_{\max }\end{array}\right]^{T}$ and $\boldsymbol{X}_{2, \min }=\left[\begin{array}{ll}\mathbf{p}_{\min } & T_{s} \mathbf{J}_{R}^{+}(\mathbf{u}) \dot{\mathbf{p}}_{\min }\end{array}\right]^{T}$. Note, that by using the differential kinematic relation in 3.13 , the constraint on the instrument tip velocity described in (3.24) can be rewritten as a constraint on the change in arc parameters $\left(\mathbf{a}_{(k)}\right)$ according to

$$
T_{s} \mathbf{J}_{R}^{+}(\mathbf{u}) \dot{\mathbf{p}}_{\min } \leq \mathbf{a}_{(k)} \leq T_{s} \mathbf{J}_{R}^{+}(\mathbf{u}) \dot{\mathbf{p}}_{\max } \forall k,
$$

where $\mathbf{J}_{R}^{+}(\mathbf{u})$ is the Moore-Penrose pseudo inverse of $\mathbf{J}_{R}(\mathbf{u})$. By adding the stacked inequality constraint, we complete the CSPCP presented in 3.33 to 3.36 .

The CSPCP is used to formulate a model based on the concept of prediction, which is described in Appendix 3.A. The prediction model is used to solve for the optimal control vector $\left(\tilde{\mathbf{v}}_{(k)}\right)$. By using predictions of the cost signal provided in (3.39) and the diagonal selection matrix described in (3.30), given the prediction interval $0 \leq j \leq N-1$, we can formulate a signal vector $\left(\tilde{\mathbf{z}}_{k}\right)$ similar to 3.52 and provide a block diagonal selection matrix $(\overline{\boldsymbol{\Gamma}})$ by

$$
\tilde{\mathbf{z}}_{(k)}=\left[\begin{array}{c}
\hat{\mathbf{z}}_{(k \mid k)} \\
\hat{\mathbf{z}}_{(k+1 \mid k)} \\
\vdots \\
\hat{\mathbf{z}}_{(k+N-1 \mid k)}
\end{array}\right] \text { and } \overline{\boldsymbol{\Gamma}}=\left[\begin{array}{cccc}
\boldsymbol{\Gamma}_{(1)} & \mathbf{0}_{5 \times 5} & \ldots & \mathbf{0}_{5 \times 5} \\
\mathbf{0}_{5 \times 5} & \boldsymbol{\Gamma}_{(2)} & \ddots & \vdots \\
\vdots & \ddots & \ddots & \mathbf{0}_{5 \times 5} \\
\mathbf{0}_{5 \times 5} & \ldots & \mathbf{0}_{5 \times 5} & \boldsymbol{\Gamma}_{(N-1)}
\end{array}\right] \text {, }
$$

respectively, which can be used to rewrite the cost-function presented in 3.28 according to

$$
J_{(\mathbf{v}, k)}=\tilde{\mathbf{z}}_{(k)}^{T} \overline{\boldsymbol{\Gamma}} \tilde{\mathbf{z}}_{(k)} .
$$

Note, that the cost-function $J_{(\mathbf{v}, k)}$ includes estimates of $\mathbf{z}_{(k)}$ using all inputs $\mathbf{v}_{(0)}, \ldots, \mathbf{v}_{(k+N-1)}$ and previous and current measurements $\mathbf{y}_{(0)}, \ldots, \mathbf{y}_{(k)}$. The predicted cost signal $\left(\tilde{\mathbf{z}}_{k}\right)$ in 3.42 can be formulated as

$$
\tilde{\mathbf{z}}_{(k)}=\tilde{\mathbf{C}}_{2} \mathbf{x}_{(k)}+\tilde{\mathbf{D}}_{21} \mathbf{e}_{(k)}+\tilde{\mathbf{D}}_{22} \tilde{\mathbf{w}}_{(k)}+\tilde{\mathbf{D}}_{23} \tilde{\mathbf{v}}_{(k)},
$$


where matrices $\tilde{\mathbf{C}}_{2}, \tilde{\mathbf{D}}_{21}, \tilde{\mathbf{D}}_{22}$ and $\tilde{\mathbf{D}}_{23}$ can be obtained according 3.54$)$ and (3.55). In addition to the constraint described in CSPCP according to (3.40), we add additional constraints to the prediction model to shape the control signal. In order to obtain a smooth and robust control action, we add a control horizon constraint to the prediction model. The equality constraint $\left(\mathbf{\Upsilon}_{(k)}=\right.$ $\mathbf{0}_{2 \times 1}$ ) on the control horizon can be described by

$$
\mathbf{v}_{(k+j \mid k)}=\mathbf{\Upsilon}_{(k)}, \text { for } N_{c} \leq j<N,
$$

where the control action is assumed to be zero after control horizon $\left(N_{c}=10\right)$, which is empirically determined. The corresponding prediction signal $\left(\tilde{\boldsymbol{v}}_{(k)}\right)$ according to 3.51 and 3.52 is given by

$$
\tilde{\boldsymbol{v}}_{(k)}=\tilde{\mathbf{C}}_{3} \mathbf{x}_{(k)}+\tilde{\mathbf{D}}_{31} \mathbf{e}_{(k)}+\tilde{\mathbf{D}}_{32} \tilde{\mathbf{w}}_{(k)}+\tilde{\mathbf{D}}_{33} \tilde{\mathbf{v}}_{(k)}=\tilde{\boldsymbol{\Upsilon}}_{(k)},
$$

where the equality constraint prediction vector is given by $\tilde{\Upsilon}_{(k)}=\mathbf{0}_{10 \times 1}$ and the corresponding matrices are described by $\tilde{\mathbf{C}}_{3}=\mathbf{0}_{10 \times 6}, \tilde{\mathbf{D}}_{31}=\mathbf{0}_{10 \times 3}, \tilde{\mathbf{D}}_{32}=$ $\mathbf{0}_{10 \times 90}$ and

$$
\tilde{\mathbf{D}}_{33}=\left[\begin{array}{ccccccc}
\mathbf{0}_{2 \times 2} & \cdots & \mathbf{0}_{2 \times 2} & \mathbf{I}_{2} & \mathbf{0}_{2 \times 2} & \cdots & \mathbf{0}_{2 \times 2} \\
\mathbf{0}_{2 \times 2} & \cdots & \mathbf{0}_{2 \times 2} & \mathbf{0}_{2 \times 2} & \mathbf{I}_{2} & \ddots & \vdots \\
\vdots & & \vdots & \vdots & \ddots & \ddots & \mathbf{0}_{2 \times 2} \\
\mathbf{0}_{2 \times 2} & \cdots & \mathbf{0}_{2 \times 2} & \mathbf{0}_{2 \times 2} & \cdots & \mathbf{0}_{2 \times 2} & \mathbf{I}_{2}
\end{array}\right]
$$

Further, a prediction vector $\left(\tilde{\boldsymbol{\chi}}_{(k)}\right)$ of the constraint signal $\left(\boldsymbol{\chi}_{(k)}\right)$ described in 3.40 can be constructed similar to 3.52 , with corresponding prediction matrices computed according to (3.54) and (3.55). The prediction of the inequality constraint is given by

$$
\tilde{\boldsymbol{\chi}}_{(k)}=\tilde{\mathbf{C}}_{4} \mathbf{x}_{(k)}+\tilde{\mathbf{D}}_{41} \mathbf{e}_{(k)}+\tilde{\mathbf{D}}_{42} \tilde{\mathbf{w}}_{(k)}+\tilde{\mathbf{D}}_{43} \tilde{\mathbf{v}}_{(k)} \leq \tilde{\boldsymbol{X}}_{(k)},
$$

which also completes the prediction model.

By minimizing the following cost-function

$$
\min _{\tilde{\mathbf{v}}} \tilde{\mathbf{z}}_{(k)}^{T} \overline{\boldsymbol{\Gamma}} \tilde{\mathbf{z}}_{(k)},
$$

subject to constraints described in $(3.46)$ and $(3.48)$, we can solve for the optimal control vector $\left(\tilde{\mathbf{v}}_{(k)}\right)$ that optimizes the CSPCP. The optimization described in 3.49 can be evaluated as a quadratic programming problem subject to constraints. For details on the derivations, we refer the reader to work of 120. We use qpOases software in $\mathrm{C}++$ to solve the quadratic programming problem online [122]. The solution is used to obtain the optimal control vector $\left(\tilde{\mathbf{v}}_{(k)}\right)$. We implement according to the receding horizon principle, where we apply $\left(\mathbf{a}_{(k)}\right)$ to steer the RADS according to the optimization. 
Chapter 3. Model predictive control of a robotically-actuated delivery sheath for beating heart compensation

\subsubsection{Hysteresis compensation}

Before the optimal solution is implemented, we compensate for hysteresis in the system. Hysteresis caused by backlash often occurs in cable driven instruments such as endoscopes and catheters 123. Hysteresis can be described by a positive or negative contact mode and a free mode. In contact mode, kinematics describes the relation between pulley angles $\left(\psi_{x}\right.$ and $\left.\psi_{y}\right)$ and RADS tip position (p), which is not known in free mode. Further, the positive and negative contact modes corresponds to the direction in which the pulleys are engaged. Hysteresis often introduces inaccuracies in the response of the instrument. Hence, we use a classical approach to reduce hysteresis in the system according to

$$
\Delta \mathbf{c}=\left[\begin{array}{ll}
\Delta \psi_{x} & \Delta \psi_{y}
\end{array}\right]^{T}+\left[\begin{array}{ll}
f_{\left(\Delta \psi_{x}\right)} & f_{\left(\Delta \psi_{y}\right)}
\end{array}\right]^{T}
$$

where $\boldsymbol{\Delta} \mathbf{c}$ is the compensated angular velocity of the pulleys $\left(\Delta \psi_{x}\right.$ and $\left.\Delta \psi_{y}\right)$ and

$$
f_{\left(\Delta \psi_{*(k)}\right)}= \begin{cases}\operatorname{sign}\left(\Delta \psi_{*(k)}\right)\left(\alpha_{*}^{+}-\alpha_{*}^{-}\right) & \begin{array}{l}
\text { If } \quad \operatorname{sign}\left(\Delta \psi_{*(k)}\right) \\
\neq \operatorname{sign}\left(\Delta \psi_{*(k-1)}\right)
\end{array} \\
0 & \text { Elsewhere }\end{cases}
$$

is the function that represents the compensation term. Note, that alternative methods for the compensation of hysteresis such as a hyperbolic tangent function could be considered 115. In order to limit chatter, we only apply the compensation term if the velocity change in direction exceeds a threshold. Compensation is provided by hysteresis parameters $\left(\alpha_{*}^{+}\right.$and $\left.\alpha_{*}^{-}\right)$, which are the positive and negative contact positions, respectively. The positive and negative contact positions are experimentally evaluated by measuring the hysteresis between pulley angles and corresponding tip locations.

\subsection{Experiments}

In this section, we present experiments performed to evaluate the MPC strategy. First, we describe the components and layout of the experimental setup used to steer the RADS. Subsequently, a pre-operative AHV model is presented and integrated with the MPC strategy. This is followed by the experimental plan and results that demonstrate the capabilities of the MPC strategy.

\subsubsection{Experimental setup and materials}

The experimental setup used to evaluate the performance of the MPC is shown in Fig. 3.8. The RADS used in our experiments is based on a cable-ring structure surrounded by a hinged-tube (DEAM Corporation, Amsterdam, The 


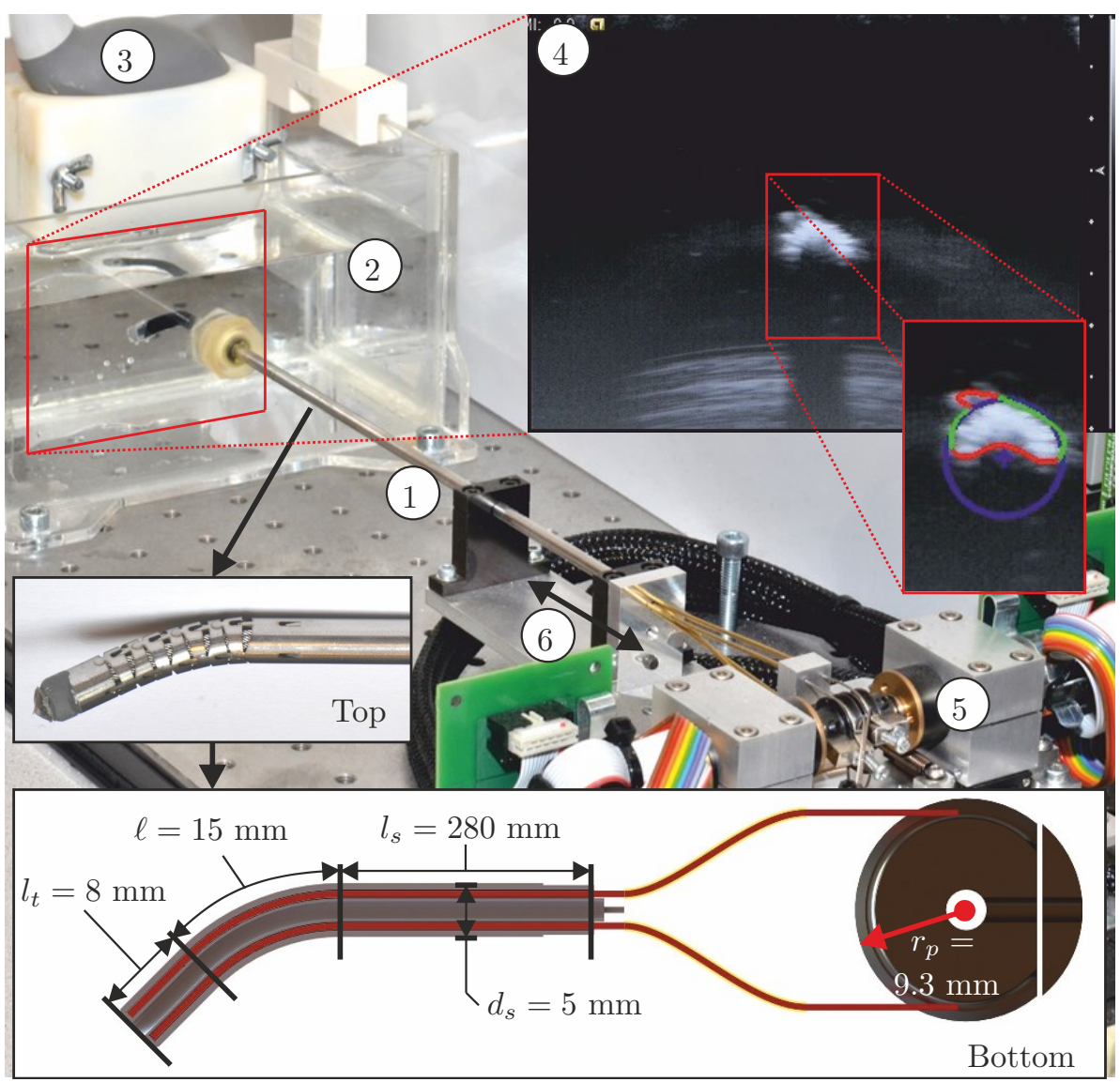

Figure 3.8: The experimental setup used to control the robotically-actuated delivery sheath (RADS) using a model predictive control strategy. (1) RADS. (2) Container filled with water in which the RADS is inserted. (3) Ultrasound transducer. (4) Ultrasound image with a radial cross-sectional view of the RADS. (5) Motors and corresponding electronics used to control the articulating tip of the RADS. (6) Translation along the longitudinal axis of the RADS in order to position the tip in the two dimensional ultrasound image plane. The top inset shows the flexible segment (articulating tip) of the RADS, which uses a hinged tube construction. The bottom inset depicts a longitudinal cross-section with dimensions of the RADS. An antagonistic configuration of a pair of tension wires (red) is actuated by a pulley-driven system. Each pair of tension wires (total of two pairs) is guided through the flexible shaft and through two incompressible brass tubes (yellow) to actuate a single degree-of-freedom of the articulating tip.

Netherlands) [124]. Two pairs of antagonistically-configured tension wires facilitate articulating tip movement of the RADS in two DOF 111. The tension wires of the RADS are driven by an ECMax22 motor with a GP32/22 gearhead (Maxon Motor, Sachseln, Switzerland). Further, a LX30 translational stage (Misumi Group Inc., Tokyo, Japan) is used to translate the RADS along 
Chapter 3. Model predictive control of a robotically-actuated delivery sheath for beating heart compensation

longitudinal axis in order to compensate for the tip motion in the out-of-imageplane direction. The maximum velocity and acceleration along the longitudinal axis are $230 \mathrm{~mm} / \mathrm{s}$ and $2800 \mathrm{~mm} / \mathrm{s}^{2}$, respectively. All motors are actuated by an Elmo Whistle 2.5/60 motor controller (Elmo Motion Control Ltd, PetachTikva, Israel). The RADS is inserted in a container filled with water in order to enable ultrasound-based tip tracking. A Siemens 18L6 transducer operating with a frequency of $16 \mathrm{MHz}$, a power level of $-4 \mathrm{~dB}$ and a scanning depth of $4 \mathrm{~cm}$ on a Siemens Acuson S2000 ${ }^{T M}$ ultrasound system (Siemens AG, Erlangen, Germany) is positioned at the tip of the RADS (in the container) to obtain ultrasound images as feedback. Note, that without any modifications to ultrasound image segmentation, the transthoracic echocardiography approach used in this study could be replaced by transesophageal echocardiography (TEE). The ultrasound images are transferred via S-video output (in-plane resolution of approximately $0.12 \mathrm{~mm}$ per pixel $)$ to a computer $\left(2.80 \mathrm{GHz}\right.$ Intel ${ }^{\circledR}$ I7, 8-GB internal memory and 64-bit Windows 7 ) with a frame rate of 25 frames per second. Further, this computer is used to implement and execute in $\mathrm{C}++$ the MPC strategy which provides control signals to the motors and electronics. The MPC strategy uses a sampling time $\left(T_{s}\right)$ of $0.04 \mathrm{~s}$. In order to anticipate the beating heart motion, we integrate an AHV model within the MPC strategy.

\subsubsection{Experimental scenarios}

A series of experiments have been conducted in order to evaluate the tracking accuracy of the MPC strategy in an integrated system using a reference signal described by in 3.25). In previous research, a model-based approach was used to steer the RADS along a circular path using $2 \mathrm{D}$ ultrasound images as feedback 111]. The results of these experiments showed that, while moving at $2 \mathrm{~mm} / \mathrm{s}$, mean positioning errors of approximately $2 \mathrm{~mm}$ along the $x$ and $y$-axes. In this study, we aim to improve these results and provide novel functionalities such as compensation for beating heart motions. Hence, we evaluate the performance using multiple scenarios such as tracking circular paths and AHV motions.

\subsubsection{Circular path}

The first set of experiments is performed in order to evaluate steering of the RADS along circular paths using the MPC strategy. Note, that no heart valve motion modeling is considered $\left(\mathbf{r}_{A H V}=\mathbf{0}\right)$ while the RADS moves along the circular paths described by reference signal $\left(\mathbf{r}_{d}\right)$. The circle has a radius of $6 \mathrm{~mm}$, while we use an articulating tip velocity of $2 \mathrm{~mm} / \mathrm{s}$. First, we evaluate a circular path without limitations on the RADS tip position. Subsequently, we limit the tip position to a maximum of $4 \mathrm{~mm}$ along the $x$-axis by integrating a state constraint to the MPC strategy (similar to $(3.40)$ ). By restricting the 
instrument motion to an allowable region, we demonstrate the ability of the MPC strategy to avoid sensitive tissue that could be present in surgery. These limitations can be integrated in the MPC strategy, allowing the controller to anticipate for the forbidden regions in order to avoid damage to sensitive tissue. In Fig. 3.9(b), the allowed area of the RADS tip is depicted green, while the forbidden region is red.

\subsubsection{Aortic heart valve motion tracking}

The second set of experiments is used to evaluate the novel designed MPC strategy to steer the RADS with a priori knowledge on the AHV motion. Although, other medical applications such as mitral valve surgery could potentially benefit from the integrated system, we demonstrate the capabilities of the MPC strategy by tracking AHV motions in the annular plane. The beating heart motions have been obtained by analyzing the pre-operative $2 \mathrm{D}$ ultrasound images as depicted in Fig. 3.6. We evaluate the performance of the MPC with a priori knowledge of the AHV motion described by tracking reference $\left(\mathbf{r}_{A H V}=\left[\begin{array}{lll}f_{x} & f_{y} & 0\end{array}\right]^{T}\right)$ obtained by $(3.31)$ and $\left(3.32\right.$ and $\mathbf{r}_{d}=\mathbf{0}$. In order to preserve the dominant features required for ultrasound image segmentation, we limit the tip velocity to $20 \mathrm{~mm} / \mathrm{s}$ in $x$ - and $y$-axes of frame $\left(\Psi_{0}\right)$ by integrating state constraints according to (3.24). A RADS tip velocity that exceeds $20 \mathrm{~mm} / \mathrm{s}$, will cause dominant features to disappear in ultrasound images. This results in unreliable segmentation of the RADS tip position. Further, we limit the maximum tip deflection to $10 \mathrm{~mm}$ in $x$ - and $y$-directions (frame $\left(\Psi_{0}\right)$ ) by $(3.23$.

\subsubsection{Experimental results}

The results of the experimental scenarios are reported in Table 3.2, while a single representative of each experiment can be found in Fig. 3.9. In order to evaluate the tip tracking accuracy of the RADS, the experiments were repeated twelve times for circular trajectories and thirty times for AHV motions. The mean absolute errors (MAE) in the tracked tip position $\left(\epsilon_{x}\right.$ and $\left.\epsilon_{y}\right)$ during trajectory tracking are reported. On average, the RANSAC algorithm of the segmentation strategy completes $819 \pm 101$ iterations (single CPU core implementation). In experiments, we observe a constant delay of approximately 200 milliseconds in obtaining the 2D ultrasound images used for feedback. Further, a compensation algorithm is used to reduce the mechanical hysteresis in the system in order to improve tip positioning accuracy.

Experiments showed tracking of a circular trajectory with MAE of $0.73 \mathrm{~mm}$ and $0.50 \mathrm{~mm}$ in the $x$ - and $y$-axes, respectively. The observed mean absolute distance error is $0.89 \mathrm{~mm}$. A slightly higher error is observed in the $x$-axis compared to the $y$-axis. This could be attributed to the difference in mechanical properties such as friction and backlash of the two uncoupled pulley 
Chapter 3. Model predictive control of a robotically-actuated delivery sheath for beating heart compensation

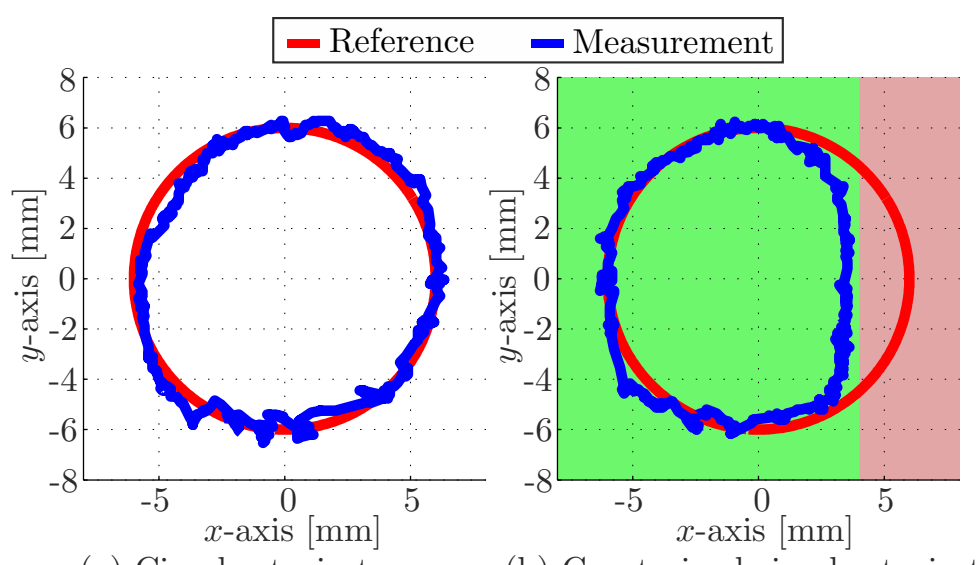

(a) Circular trajectory

(b) Constrained circular trajectory

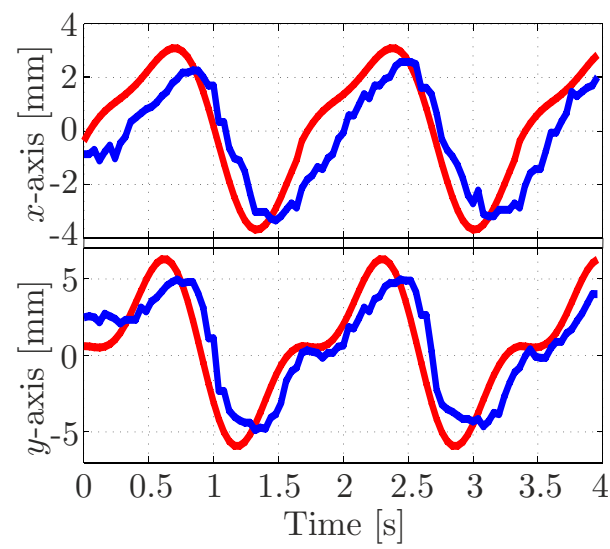

(c)AHV motion

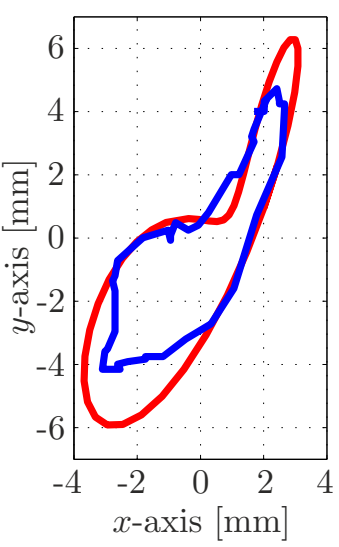

(d) 2DAHV motion

Figure 3.9: Representative experimental model-predictive control results of the articulating tip of the robotically-actuated delivery sheath during tracking of (a) circular reference path $\left(\mathbf{r}_{\mathbf{d}}\right)$ and $\left(\mathbf{r}_{\mathbf{A H V}}=\mathbf{0}\right),(\mathrm{b})$ constrained circular reference path $\left(\mathbf{r}_{\mathbf{d}}\right)$ and $\left(\mathbf{r}_{\mathbf{A H V}}=\mathbf{0}\right)$ and (c and d) aortic heart valve (AHV) motion trajectories according to $\mathbf{r}_{\mathbf{A H V}}=\left[\begin{array}{lll}f_{x} & f_{y} & 0\end{array}\right]^{T}$ and $\mathbf{r}_{\mathbf{d}}=\mathbf{0}$. The red line trajectory represents the reference path $(\mathbf{r})$ described in 3.25 , while the blue line represents the actual path $\left(\mathbf{y}=\left[\begin{array}{lll}p_{x} & p_{y} & 0\end{array}\right]^{T}\right)$ followed by the articulating tip.

systems (each control a single DOF). Nonetheless, the results show a significant improvement in tracking performance compared to the mean positioning errors of approximately $2 \mathrm{~mm}$ along the $x$ - and $y$-axes demonstrated in previous work 111]. Further, in the experiments that demonstrate the circular trajectory in which the instrument motion is limited, we observe a MAE of $0.74 \mathrm{~mm}$ and $0.61 \mathrm{~mm}$ in the $x$ - and $y$-axes, respectively. The corresponding mean absolute distance error is $0.97 \mathrm{~mm}$. The observed error correspond to the results 
Table 3.2: Experimental results of the robotically-actuated delivery sheath tip tracking for circular paths and aortic heart valve motions using the model-predictive control. The mean absolute distance error $(\epsilon)$ and position errors $\left(\epsilon_{x}\right.$ and $\left.\epsilon_{y}\right)$ along the $x$ - and $y$-axes are provided. Further, the standard deviation for $N_{r}$ repetitions is reported.

\begin{tabular}{|c|c|c|c|c|}
\hline Case & $N_{r}$ & $\begin{array}{c}\epsilon_{x} \\
{[\mathrm{~mm}]}\end{array}$ & $\begin{array}{c}\epsilon_{y} \\
{[\mathrm{~mm}]}\end{array}$ & $\begin{array}{c}\epsilon \\
{[\mathrm{mm}]}\end{array}$ \\
\hline \hline Circle & 12 & $0.73 \pm 0.03$ & $0.50 \pm 0.10$ & $0.89 \pm 0.08$ \\
\hline Constrained circle & 12 & $0.74 \pm 0.11$ & $0.61 \pm 0.17$ & $0.97 \pm 0.17$ \\
\hline Aortic heart valve & 30 & $1.06 \pm 0.43$ & $1.29 \pm 0.38$ & $1.68 \pm 0.53$ \\
\hline
\end{tabular}

of a circular trajectory without instrument limitations. Hence, we demonstrate that the MPC strategy is capable of avoiding areas that could potentially be sensitive tissue without degrading performance. By tracking AHV motions, we observe a MAE of $1.06 \mathrm{~mm}$ and $1.29 \mathrm{~mm}$ in the $x$-and $y$-axes, respectively. The observed mean absolute distance error is $1.68 \mathrm{~mm}$. A higher error is observed in tracking AHV motions compared to circular trajectories. This could be explained by the fast moving AHV motions that impedes the accuracy of tracking. Further, we observe a decrease in tracking accuracy along the $y$-axis for AHV motions. The decrease in tracking accuracy could be explained by the instrument velocity which is limited to $20 \mathrm{~mm} / \mathrm{s}$ in MPC in order to enable RADS detection in ultrasound images. The MPC strategy considers both the tip velocity limitation and AHV motion in order to optimize tracking accuracy.

Further, the ultrasound measurement delay introduced in the feedback signal of the MPC strategy is among the main contributer to the tracking error. This measurement delay is considered to be the combined result of prepoccessing within the ultrasound system, transferring images to the computer using a capturing device and instrument segmentation. However, it can not be ruled out that tracking accuracy is affected by (non)-linear friction, tendon elongation or crosstalk between the two tendon pairs (i.e., controlling one tendon pair influences the other tendon pair). Note, that we evaluate our system using pre-operative patient data which leads to simplifications with respect to heart-rate variability. However, these simplifications could potentially be eliminated by administering medicine that reduce the heart rate and apply over pacing to effectively control the patient's heart rate in a predictable manner. This improves the ability to anticipate the AHV motion. Nonetheless, our method demonstrates tracking of AHV motions based on pre-operative ultrasound patient data. This indicates that our system, with further development, could provide cardiac motion compensation to a wide class of cardiovascular applications performed without a heart-lung machine. 
Chapter 3. Model predictive control of a robotically-actuated delivery sheath for beating heart compensation

\subsection{Conclusions and future work}

In this study, we present and evaluate a novel RADS capable of autonomously and accurately compensating for AHV motions by using an MPC strategy. We develop and incorporate kinematic models of the RADS within the MPC strategy. In order to accurately evaluate the RADS tip position, we use a clinically-available $2 \mathrm{D}$ ultrasound transducer which is orientated perpendicular to the tip of the RADS. An on-line segmentation algorithm is developed in order to provide feedback of the RADS tip position for the MPC strategy. Preoperative ultrasound images of a patient are used to evaluate the AHV motion. Further, mechanical hysteresis is addressed by a compensation algorithm in order to improve the tip positioning accuracy. The novel integrated system is capable of controlling the articulating tip of the RADS in order to compensate for AHV motions. In experiments, we demonstrate evidence that the RADS tracks the AHV motions with a mean absolute distance error for AHV motions of $1.68 \mathrm{~mm}$. Hence, we potentially improve and enable new applications in cardiovascular surgery performed without a heart-lung machine.

In future work, we intend to address accuracy problems introduced by measurement delay and instrument friction. Electro-magnetic instrument tip tracking will be integrated in order to reduce feedback delay and to improve robustness in control. The prototype device used in this study will be replaced by a flexible steerable catheter in order to enable applications in ablation, aortic and mitral valve surgery. Further, we plan to combine instrument and intra-operative AHV motion segmentation of $2 \mathrm{D}$ and $3 \mathrm{D}$ ultrasound images in order to improve the tracking performance in a clinically relevant scenario. We continuously aim to improve the robustness and accuracy of the integrated system. Nonetheless, the presented framework for modeling, imaging and control is applicable to a range of continuum-style robots and catheters. Our current study evaluates compensation of AHV motions using an MPC strategy. Hence, we demonstrate feasibility and potential for steerable catheters to compensate cardiac motions in cardiovascular interventions such as aortic and mitral valve surgery. 


\section{Nomenclature}

$A_{c} \quad$ Set of detected edge points

$G \quad$ Rate of convergence feedback gain

$H_{3} \quad$ Set of three randomly selected edge points

$H_{r} \quad$ Heart rate

$J \quad$ Generalized predictive control cost

$J_{c} \quad$ Random sampling consensus cost

$N \quad$ Prediction horizon

$N_{c} \quad$ Control horizon

$N_{m} \quad$ Minimum cost-horizon

$N_{r} \quad$ Number of experimental repetitions

$R^{2} \quad$ Data fitting discrepancy

$S_{c} \quad$ Random sampling consensus set (inliers)

$S_{r} \quad$ Sample rate Fourier series

$T_{s} \quad$ Sampling time

$\Delta \psi_{*} \quad$ Angular pulley velocity, where $* \in\{x, y\}$

$\Psi_{*} \quad$ Coordinate system, where $* \in\{0, i, t, u\}$

$\alpha_{*}^{+} \quad$ Positive contact positions, where $* \in\{x, y\}$

$\alpha_{*}^{-} \quad$ Negative contact positions, where $* \in\{x, y\}$

$\boldsymbol{X}, \boldsymbol{X}_{1}, \boldsymbol{X}_{2}$ Inequality constraints $\left(\boldsymbol{X}_{1}, \boldsymbol{X}_{2} \in \mathbb{R}^{5}, \boldsymbol{X} \in \mathbb{R}^{10}\right)$

$\Delta c \quad$ Compensated angular pulley velocity $\left(\Delta c \in \mathbb{R}^{2}\right)$

$\chi, \chi_{1}, \chi_{2}$ Inequality constraint signals $\left(\chi_{1}, \chi_{2} \in \mathbb{R}^{5}, \chi \in \mathbb{R}^{10}\right)$

$\boldsymbol{\lambda}$ Control input weighting matrix $\left(\boldsymbol{\lambda} \in \mathbb{R}^{2 \times 2}\right)$

$\boldsymbol{v} \quad$ Equality constraint signal $\left(\boldsymbol{v} \in \mathbb{R}^{2}\right)$

$\delta_{*} \quad$ Tendon displacement, where $* \in\{x, y\}$

$\dot{\mathbf{p}}_{\text {min }}, \dot{\mathbf{p}}_{\max }$ Inequality constraint lower and upper velocity bound $\left(\dot{\mathbf{p}}_{\min }, \dot{\mathbf{p}}_{\max } \in \mathbb{R}^{3}\right)$

$\ell \quad$ Arc length

$\epsilon \quad$ Mean absolute tip distance error

$\epsilon_{*} \quad$ Mean absolute error in the tracked tip position, where $* \in\{x, y\}$

$\epsilon_{t h} \quad$ Error tolerance

$\kappa \quad$ Arc curvature

A, B, C, D Multiple-input and multiple-output state space realization of the CSPCP

$\mathbf{H}_{i}^{0} \quad$ Homogeneous transformation matrix expressing the intermediate frame in the reference frame $\left(\mathbf{H}_{i}^{0} \in S E 3\right)$

$\mathbf{H}_{t}^{0} \quad$ Homogeneous transformation matrix expressing the tip frame in the reference frame $\left(\mathbf{H}_{t}^{0} \in S E 3\right)$

$\mathbf{H}_{t}^{i} \quad$ Homogeneous transformation matrix expressing the tip frame in the intermediate frame $\left(\mathbf{H}_{t}^{i} \in S E 3\right)$

$\mathbf{L}_{t}^{i} \quad$ Rigid link section by a translation along the $z$-axis of the intermediate frame $\left(\mathbf{L}_{t}^{i} \in \mathbb{R}^{3}\right)$

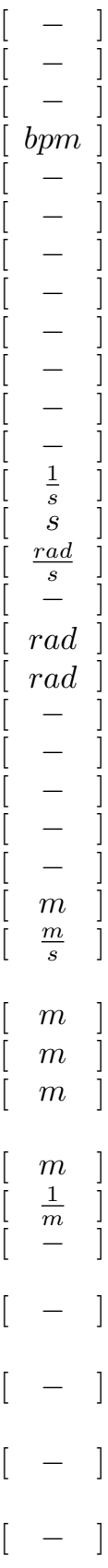


Chapter 3. Model predictive control of a robotically-actuated delivery sheath for beating heart compensation

$\boldsymbol{\Gamma} \quad$ Diagonal selection matrix $\left(\boldsymbol{\Gamma} \in \mathbb{R}^{5 \times 5}\right)$

$\mathbf{\Upsilon}$ Equality constraint $\left(\mathbf{\Upsilon} \in \mathbb{R}^{2}\right)$

a Variations in arc parameters $\left(\mathbf{a}=\left[\begin{array}{ll}\Delta \phi & \Delta \kappa\end{array}\right]^{T} \in \mathbb{R}^{2}\right)$

d The MPC disturbance $\left(\mathbf{d} \in \mathbb{R}^{3}\right)$

e Zero mean white noise $\left(\mathbf{e}, \hat{\mathbf{e}} \in \mathbb{R}^{2}\right)$

$\mathbf{e}_{r r} \quad$ The tip position error $\left(\mathbf{e}_{r r} \in \mathbb{R}^{3}\right)$

$\mathbf{o}_{t} \quad$ Origin of the articulating tip frame $\left(\mathbf{o}_{t} \in \mathbb{R}^{3}\right)$

$\mathbf{p}, \mathbf{p}_{t}^{0}$ Tip position expressed in the reference frame $\left(\mathbf{p}, \mathbf{p}_{t}^{0} \in \mathbb{R}^{3}\right)$

$\mathbf{p}_{\min }, \mathbf{p}_{\max }$ Inequality constraint lower and upper position bound $\left(\mathbf{p}_{\text {min }}, \mathbf{p}_{\text {max }} \in \mathbb{R}^{3}\right)$

$\mathbf{r}, \mathbf{r}_{t}^{0}$ Referenced tip position expressed in the reference frame $\left(\mathbf{r}, \mathbf{r}_{t}^{0} \in \mathbb{R}^{3}\right)$

$\mathbf{r}_{A H V}$ Aortic heart valve annulus motion reference $\left(\mathbf{r}_{A H V} \in \mathbb{R}^{3}\right)$

$\mathbf{r}_{d} \quad$ Desired tip position $\left(\mathbf{r}_{d} \in \mathbb{R}^{3}\right)$

$\mathbf{u} \quad$ Arc parameters, $\mathbf{u}=\left[\begin{array}{ll}\phi & \kappa\end{array}\right]^{T} \in \mathbb{R}^{2}$

v Control input signal $\left(\mathbf{v} \in \mathbb{R}^{2}\right)$

$\mathbf{w} \quad$ The MPC external signal $\left(\mathbf{w}=\left[\begin{array}{ll}\mathbf{d} & \mathbf{r}\end{array}\right]^{T} \in \mathbb{R}^{6}\right)$

$\mathbf{x} \quad$ The MPC state variable $\left(\mathbf{x}=\left[\begin{array}{ll}\mathbf{p} & \mathbf{a}\end{array}\right]^{T} \in \mathbb{R}^{5}\right)$

$\mathbf{x}_{c} \quad$ Edge point obtained from Canny edge detector $\left(\mathbf{x}_{c} \in \mathbb{R}^{2}\right)$

$\mathbf{y} \quad$ MPC tip position $\left(\mathbf{y} \in \mathbb{R}^{3}\right)$

$\mathbf{z} \quad$ Cost signal $\left(\mathbf{z} \in \mathbb{R}^{3}\right)$

$\omega, \omega_{*}$ The Fourier series frequencies, where $* \in\{x, y\}$

$\phi \quad$ Arc plane angle

$\phi_{i} \quad$ Individual tendon angle, where $i=1, \cdots, 4$

$\psi_{*} \quad$ Pulley angle, where $* \in\{x, y\}$

$\theta \quad$ Arc bend angle

$a_{*}, b_{*}$ The Fourier series coefficients

$d_{b} \quad$ Distance between backbone and tendons

$e \quad$ Estimation error

$f_{*} \quad$ AHV reference position, where $* \in\{x, y\}$

$k \quad$ Discrete time variable

$l_{i} \quad$ Tendon lengths, where $i=1, \cdots, 4$

$l_{t} \quad$ Rigid link length

$m_{c} \quad$ Algebraic circle model parameters

$n \quad$ Number of random sampling consensus iterations

$p_{*} \quad$ Tip position, where $* \in\{x, y, z\}$

$r \quad$ Arc radius

$r_{*} \quad$ Referenced tip position, where $* \in\{x, y, z\}$

$r_{p} \quad$ Pulley radius

$t_{i} \quad$ Tension wires, where $i=1, \cdots, 4$

Note, that we use $\hat{*}$ and $\tilde{*}$ in order to describe an estimate or a prediction of $(*)$, respectively. 


\section{A Concept of prediction}

Based on the model description (3.34), the concept of prediction can be introduced according to

$$
\tilde{\mathbf{s}}_{p,(k)}=\tilde{\mathbf{C}}_{p} \mathbf{x}_{(k)}+\tilde{\mathbf{D}}_{p 1} \mathbf{e}_{(k)}+\tilde{\mathbf{D}}_{p 2} \tilde{\mathbf{w}}_{(k)}+\tilde{\mathbf{D}}_{p 3} \tilde{\mathbf{v}}_{(k)},
$$

where any signal $\mathbf{s}_{p,(k)}$ as presented in 3.34 can be used to formulate predictions at each time instant $(k)$, that considers all signals over horizon $N$. The prediction of signal $\mathbf{s}_{p,(k)}$ is given by signal vector $\tilde{\mathbf{s}}_{p,(k)}$ according to

$$
\tilde{\mathbf{s}}_{p,(k)}=\left[\begin{array}{c}
\hat{\mathbf{s}}_{p,(k \mid k)} \\
\hat{\mathbf{s}}_{p,(k+1 \mid k)} \\
\vdots \\
\hat{\mathbf{s}}_{p,(k+N-1 \mid k)}
\end{array}\right] \text {. }
$$

The prediction presented in (3.51) is based on future control and reference signals described by signal vectors

$$
\tilde{\mathbf{v}}_{(k)}=\left[\begin{array}{c}
\hat{\mathbf{v}}_{(k \mid k)} \\
\hat{\mathbf{v}}_{(k+1 \mid k)} \\
\vdots \\
\hat{\mathbf{v}}_{(k+N-1 \mid k)}
\end{array}\right] \text { and } \quad \tilde{\mathbf{w}}_{(k)}=\left[\begin{array}{c}
\hat{\mathbf{w}}_{(k \mid k)} \\
\hat{\mathbf{w}}_{(k+1 \mid k)} \\
\vdots \\
\hat{\mathbf{w}}_{(k+N-1 \mid k)}
\end{array}\right],
$$

respectively. Note, that $\tilde{\mathbf{w}}_{(k)}$ contains a priori knowledge of the AHV position. We can obtain matrices $\tilde{\mathbf{C}}_{p}, \tilde{\mathbf{D}}_{p 1}, \tilde{\mathbf{D}}_{p 2}$ and $\tilde{\mathbf{D}}_{p 3}$ by using successive substitution of the state equation (3.33) into (3.34) which yields

$$
\tilde{\mathbf{C}}_{p}=\left[\begin{array}{c}
\mathbf{C}_{p} \\
\mathbf{C}_{p} \mathbf{A} \\
\mathbf{C}_{p} \mathbf{A}^{2} \\
\vdots \\
\mathbf{C}_{p} \mathbf{A}^{N-1}
\end{array}\right], \tilde{\mathbf{D}}_{p 1}=\left[\begin{array}{c}
\mathbf{D}_{p 1} \\
\mathbf{C}_{p} \mathbf{B}_{1} \\
\mathbf{C}_{p} \mathbf{A} \mathbf{B}_{1} \\
\vdots \\
\mathbf{C}_{p} \mathbf{A}^{N-2} \mathbf{B}_{1}
\end{array}\right]
$$

and

$$
\tilde{\mathbf{D}}_{p q}=\left[\begin{array}{ccccc}
\mathbf{D}_{p q} & 0 & \cdots & 0 & 0 \\
\mathbf{C}_{p} \mathbf{B}_{q} & \mathbf{D}_{p q} & \ddots & \vdots & \vdots \\
\mathbf{C}_{p} \mathbf{A B}_{q} & \mathbf{C}_{p} \mathbf{B}_{q} & \ddots & 0 & 0 \\
\vdots & & \ddots & \mathbf{D}_{p q} & 0 \\
\mathbf{C}_{p} \mathbf{A}^{N-2} \mathbf{B}_{q} & & \cdots & \mathbf{C}_{p} \mathbf{B}_{q} & \mathbf{D}_{p q}
\end{array}\right]
$$

where subscript $q=2,3$ is used to indicate the corresponding matrix to complete the prediction. The concept of prediction is used to provide a prediction model of the CSPCP signals described in 3.34, 3.35) and (3.36). 
Chapter 3. Model predictive control of a robotically-actuated delivery sheath for beating heart compensation 


\title{
CHAPTER 4 \\ Sensor-Guided Stabilization of a Steerable and Flexible Catheter Tip for Beating Heart Mitral Valve Motions
}

\begin{abstract}
A mitral valve (MV) repair strategy that uses minimally invasive surgical techniques could significantly reduce patient trauma compared to an open heart procedure via sternotomy. Reduced trauma enables fast recovery and could provide treatment to high-risk patients. However, minimally invasive techniques are often impeded by limited vision and dexterity of the instrument at the treatment location, while the absence of cardiopulmonary bypass requires the clinician to cope with the beating heart motions. By sensor-guided stabilization of the instrument, a virtually-still treatment location could be provided, which enables the clinician to perform surgery as if the heart was stopped. In this study, we present and evaluate electromagnetic (EM) and ultrasound (US)-guided steerable and flexible catheter (SFC) tip stabilization methods in order to assist the clinician and to potentially improve the capabilities of minimal invasive MV repair surgery. Our work involves developing a novel SFC tip stabilization method in a realistic and functional MV model embedded in a heart system. The heart system is attached to a six degrees-of-freedom Stewart platform, which is used to reproduce beating heart MV motions based on pre-operative patient data. Further, we developed a novel catheter steering module capable of robot actuation of a wide range of tendon-driven catheters. In order to demonstrate tip stabilization for beating heart MV motions, the SFC is controlled in closed-loop, which uses EM sensing and US imaging to provide instrument feedback. Experimental results showed a mean absolute distance error of $0.57 \mathrm{~mm}$ and $1.39 \mathrm{~mm}$ for EM- and US-guided SFC tip stabilization methods, respectively. The presented methods for stabilization of the SFC tip in the beating heart could be applicable to a wide variety of existing and potential future cardiovascular interventions.
\end{abstract}

This chapter is submitted for publication as

G.J. Vrooijink and S. Misra, Sensor-Guided Stabilization of a Steerable and Flexible Catheter Tip for Beating Heart Mitral Valve Motions, IEEE Transactions on Medical Robotics and Bionics (under review). 
Chapter 4. Sensor-Guided Stabilization of a Steerable and Flexible Catheter Tip for Beating Heart Mitral Valve Motions

while preliminary work is described in

125 G.J. Vrooijink, M.P. Jansen, M.L. Tolhuisen, J.G. Grandjean, and S. Misra, Ultrasound-guided stabilization of a robotically-actuated delivery sheath (RADS) for beating heart mitral valve motions, in Proceedings of the IEEE International Conference on Biomedical Robotics and Biomechatronics (BioRob), Singapore, pages 73-79, June 2016 (Best Conference Paper Award). 


\subsection{Introduction}

Traditionally, open heart surgery via sternotomy is used to facilitate repair strategies in order to provide treatment to a deceased mitral valve (MV). A repair strategy aims to restore the natural MV functionality in order to preserve hemodynamics, reduce the risk of embolism and minimize long-term anticoagulation, which is beneficial for the patient's quality of life after surgery [13. MV repair is often associated with improved patient survival compared to prosthetic valve implantation [12. A repair strategy often requires complex cardiopulmonary bypass surgery with careful intra-operative decision making. Direct and superior surgical access to the deceased MV is obtained via sternotomy, which goes at the expense of severe patient trauma. Hence, patients with comorbidities, which are considered a high risk, are often excluded [14, 17.

As an alternative to open heart surgery, minimally invasive surgery (MIS) could provide MV repair treatment, which enables treatment to high-risk patients and results in shorter recovery times [15]. However, challenges such as accessibility, vision and dexterity at the treatment location limits the scale of MIS [18]. In addition to the aforementioned challenges, MV repair treatment without the use of cardiopulmonary bypass is often impeded by beating-heart motions. The complex and repetitive beating-heart motions pose a significant challenge for a clinician, whose ability to track, significantly deteriorates for heart rates up to 60 beats-per-minute (BPM) 19 .

The use of steerable and flexible catheters (SFC) could provide an aid to the aforementioned challenges and facilitate MIS MV repair treatment [16]. Technological developments in robotically-controlled catheters could offer improved surgical access and dexterity at the treatment location, while patient trauma is minimized. Note, that experience obtained from clinical practice is used to quantify the requirements for the tendon driven catheters and instruments 124, 126, 127. In this study, we use such a tendon driven instrument developed by DEAM Corporation (Amsterdam, the Netherlands). Further, tasks such as stabilization of the catheter tip for beating heart motions could be obtained by robotic control. Instrument tip stabilization for beating heart motions at the treatment location could provide a scenario as if the heart was stopped, which allows the clinician to mainly focus on the MV repair procedure.

\subsubsection{Related work}

The potential use of SFCs such as magnetically-actuated catheters for MIS has been demonstrated by several research groups [128, 129]. These catheters could provide dexterity and instrument stabilization for beating heart motions, which is desired in MIS MV repair. However, a limited operational workspace and the integration of external auxiliary equipment such as electromagnetic coils or permanent magnets used to enable magnetic catheter steering, poses a significant challenge in clinical practice. 
Tendon or push-and-pull rod driven catheters, often controlled in a master and slave configuration, have been evaluated in cardiovascular navigation and positioning tasks 130 135. In these studies, navigation and instrument positioning is often assisted by medical imaging such as ultrasound (US). Further, recent work documents the use of SFCs to perform a specific surgical tasks such as automatic pointing of the US imaging catheter, tissue removal and patent foramen ovales closure 80,136, 138. However, instrument stabilization for beating heart motions at the treatment location was not considered in these studies.

Applications for stabilizing instruments on the beating heart surface has been documented 70,72,74, 139]. The majority of these studies use stereoscopic imaging to visualize both instrument and target, which is not suitable for internal beating heart surgery. Only a limited number of studies describe catheter stabilization within the beating heart using medical imaging such as US 75, 78, 81. Compared to other medical imaging modalities, ultrasound offers a safe and accessible method to visualize both instrument and treatment locations during the intervention. Note, that computed tomography scans exposes both patient and clinician to a high doses of radiation during the intervention, while magnetic resonance imaging requires the instruments to be made of nonmagnetic and dielectric materials 90]. Although, instrument tip stabilization for beating heart motions was demonstrated, motion compensation was limited to the longitudinal axis of the catheter. A wide variety of future and existing MV repair procedures could benefit from improved dexterity at the treatment location and instrument stabilization orthogonal to the catheter tip as described in research 18.

\subsubsection{Contributions}

In this study, we contribute an integrated system which uses a SFC to potentially improve the capabilities of minimal invasive MV repair surgery (Fig.4.1). Further, we develop a novel robotic system that stabilizes the SFC tip for MV motions during the cardiac cycle. Such a system could potentially be considered as an alternative to cardiopulmonary bypass surgery by enabling minimally invasive MV repair procedures on a beating heart.

In this study, we design a novel catheter steering module capable of robot actuation of a wide range of tendon driven catheters by using a pivoting disc controlled by two linear motors. A prototype SFC is attached to the steering module, which we equip with electromagnetic (EM) tracking sensors at the instrument base and tip. The EM tracking sensors integrated in the SFC are used to provide instrument feedback. The SFC and steering module are experimentally evaluated in a realistic and functional acrylic heart system with an embedded MV model. The acrylic heart system attached to a 6DOF Stewart platform is used to reproduced beating heart MV motions based on patient data obtained from pre-operative US and magnetic resonance (MR) imaging [125. 


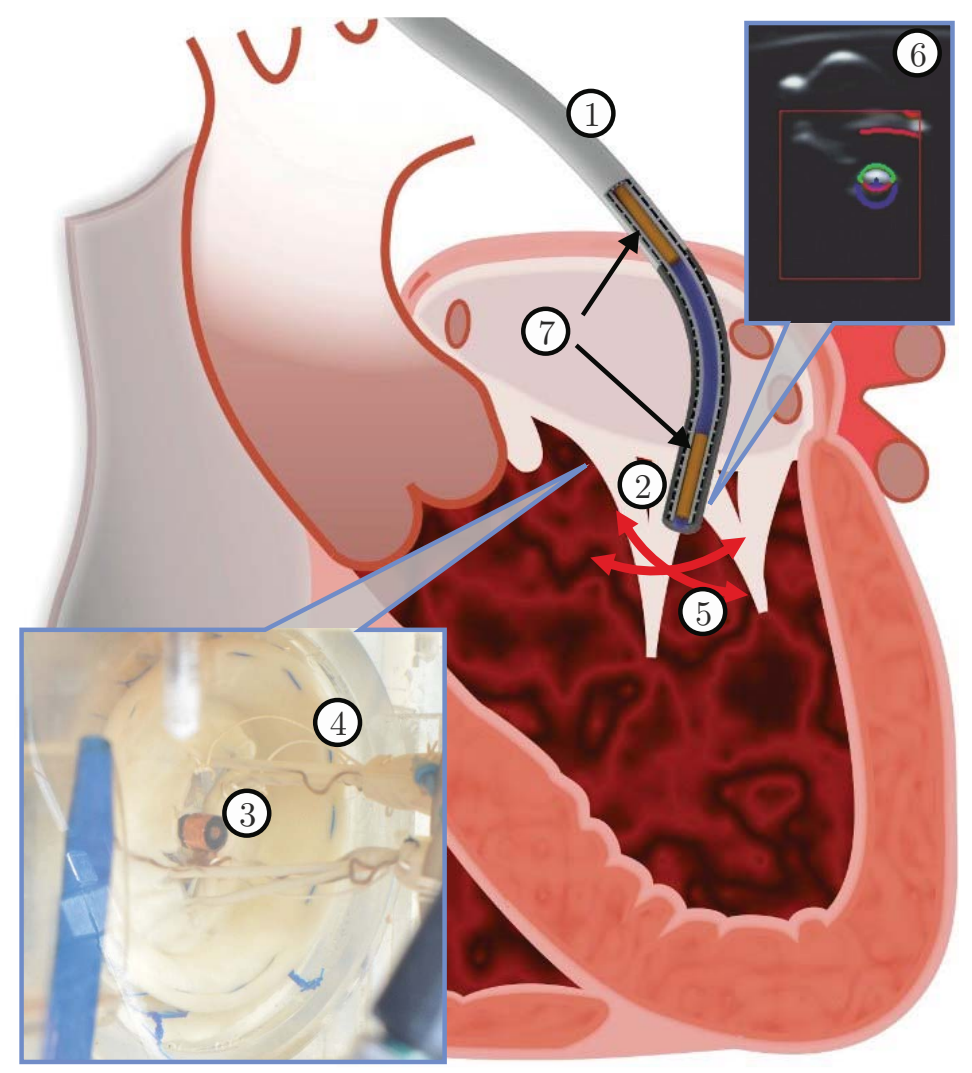

Figure 4.1: A steerable and flexible catheter (SFC) (1) is inserted into the left atrium of the heart. The SFC tip shown in (2) and depicted in the bottom-left inset (3) is introduced into the mitral valve (MV) model shown in (4). The articulating SFC tip is stabilized for beating MV motions (5) within the MV annulus. Motions of the SFC are tracked by ultrasound segmentation (6) and Electro Magnetic (EM) sensors (7) embedded at the base of the steerable segment and instrument tip, which are used for feedback in closed-loop control. The ultrasound transducer (not shown) is placed in the vicinity of the MV and the SFC.

Further, in this study, we expand the acrylic heart system with a pulsatile pump to enable fluid flow. Thus, enabling functional opening and closing of the embedded MV. In order to demonstrate tip stabilization for beating heart MV motions, the catheter is controlled in closed-loop, which uses EM sensing and US imaging to provide instrument feedback.

To the best of our knowledge, we are the first to stabilize the tip of a steerable and flexible catheter in a functional MV model by using EM and US tracking. The proposed method of autonomous catheter tip stabilization for beating heart motions could offer assistance to the clinician during surgery in order to reduce work load. Future and existing applications in MIS such as 
Chapter 4. Sensor-Guided Stabilization of a Steerable and Flexible Catheter Tip for Beating Heart Mitral Valve Motions

MV repair, ablation and cardiovascular biopsies could benefit from improved accessibility and dexterity offered by the integrated system.

\subsection{Methods}

In this section, we describe methods for stabilization of the SFC tip as it is subjected to beating heart MV motions. In section 4.2.1, we describe the experimental setup. In section 4.2.2, we elaborate on the SFC used in this study. In section 4.2.3 we present the catheter steering module used to control the SFC in closed-loop. Subsequently, in section 4.2.4 we provide details about SFC tracking, which is facilitated by EM sensors and US segmentation. In section 4.2.5 we elaborate on our closed-loop control strategy. Further, in section 4.2.6 we describe the pre-operative patient data used to reproduce MV motions in experimental evaluation. Note that, in the derivation presented, we denote for notational simplicity: $c_{(*)}=\cos (*)$ and $s_{(*)}=\sin (*)$.

\subsubsection{Experimental setup}

An overview of the experimental setup can be found in Fig. 4.2, The experiment setup comprises of a SFC controlled in closed-loop by using a catheter steering module. The tip of the SFC with integrated EM tracking sensors, is inserted into an acrylic heart system that contains a functional and realistic MV model. The acrylic heart system is attached to a 6DOF Stewart platform in order to reproduce beating heart MV motions based on pre-operative patient data. We integrate a pulsatile pump in order to provide a volume displacement that enables opening and closing of the MV model. In experimental evaluation, the catheter steering module is used for closed-loop control of the SFC in order to provide instrument tip stabilization for the beating heart MV motions.

The catheter steering module is used to steer the SFC as shown in Fig. 4.2 The catheter steering module uses a pivoting disc in order to manipulate the tendons of the SFC, which enables the articulating tip to be positioned in two dimensions (2D). The disc pivoting and the SFC tip bending direction are directly related, while the disc pivoting angle is proportional to the SFC tip bend angle. By considering a pivoting disc, the instrument tip actuation is independent of the SFC tendon configuration. Hence, any desirable SFC using tendon actuation, could be connected to the pivoting disc of the catheter steering module. The disc pivoting angle and direction is controlled by two LM1247-020-02C (Dr. Fritz Faulhaber GMBH \& CO. KG, Schönaich, Germany) linear motors. Note, that by using linear motors, the SFC tip contact forces could potentially be perceived by the linear motor shaft due to its backdrivability.

The SFC depicted in Fig. 4.2 is a tendon-driven instrument (DEAM Corporation, Amsterdam, the Netherlands), which comprises of a flexible shaft, a steerable segment and a tip segment. The articulating tip of the SFC is con- 


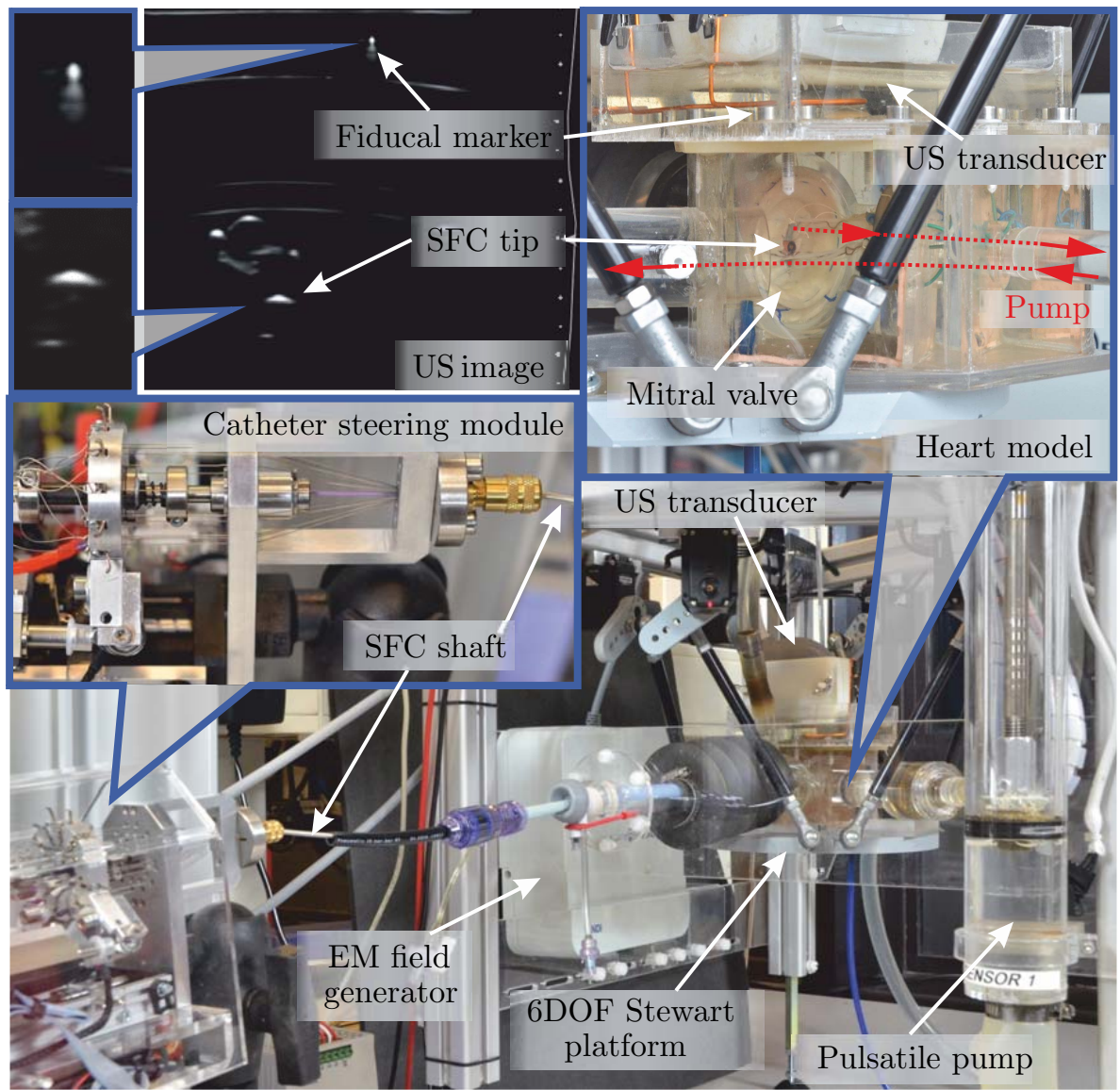

Figure 4.2: An overview of the experimental setup used to demonstrate steerable and flexible catheter (SFC) tip stabilization for mitral valve (MV) motions. An acrylic heart system (bottom right inset) equipped with a realistic and function mitral valve (MV) model is mounted on a six degrees-of-freedom (DOF) Stewart platform. The Stewart platform is used to reproduce beating heart motions based on pre-operative patient data, while a pulsatile pump provides a fluid flow (indicated by the red arrows) in order to enable opening and closing of the MV model. The tip of the SFC is inserted in the MV model, while the SFC tendons are connected to the disc of the catheter steering module as depicted in the left inset. The catheter steering module disc orientation is controlled by two linear motors and enables the articulating SFC tip to be positioned in two dimensions. An electromagnetic (EM) field generator is incorporated to provide SFC tip feedback, which is used for closedloop control. Further, a two-dimensional ultrasound (US) transducer is positioned along the MV annulus, which provides a radial cross-sectional view (top left inset) of the SFC tip and the fiducal marker. US segmentation of the SFC tip and fiducal marker are used for feedback in closed-loop control and provide a reference for the MV motion path, respectively.

trolled in $2 \mathrm{D}$ by actuation of twelve tendons, which are attached to the pivoting disc of the catheter steering module. The working channel of the SFC has a 
Chapter 4. Sensor-Guided Stabilization of a Steerable and Flexible Catheter Tip for Beating Heart Mitral Valve Motions

diameter of $0.8 \mathrm{~mm}$, in which we integrated two EM tracking sensors (Northern Digital Inc., Ontario, Canada). A 5DOF EM tracking sensor is at the base of the steerable segment, while a second 6DOF EM tracking sensor is attached to the tip segment.

The SFC is inserted into an acrylic heart system that contains a functional and realistic MV model (LifeLike BioTissue Inc., London, Canada) as shown in the inset of Fig. 4.2. Note, that the MV model used in this study is implemented with an anterior and posterior middle prolapse. The acrylic heart system is attached to a $6 \mathrm{DOF}$ Stewart platform, which is used to reproduce beating heart MV motions observed in pre-operative patient data. The MV motions are obtained from patients using pre-operative MR and US images. For more details we refer the reader to previous work [125].

In order to enable opening and closing of the MV model, we developed a pulsatile pump. The pulsatile pump is used to displace approximately $50 \mathrm{ml}$ of fluid within the acrylic heart system. The pulsatile flow enables the realistic MV model and the artificial aortic valve to successively open and close. The pump comprises of a piston, which is driven by an ECMax22 motor connected to a GP22S spindle drive (Maxon Motor, Sachseln, Switzerland). The pump has a displacement accuracy of approximately $0.1 \mathrm{ml}$.

In order to provide feedback for closed-loop control, we use a Siemens Acuson S2000 ${ }^{\mathrm{TM}}$ US system (Siemens AG, Erlangen, Germany) to obtain images of the SFC tip and the MV model. A Siemens 18L6 US transducer provides a radial cross-sectional view of the SFC tip and the MV annulus as shown in the inset of Fig. 4.2. Note, that the US transducer is stationary, which leads to simplification with respect to a clinical scenario. Further, in clinical practice, the transthoracic echocardiogram (TTE) approach used in this study could be replaced by a transesophageal echocardiography (TEE) without modifications to our segmentations strategies. The US images are obtained using a frequency of $16 \mathrm{Mhz}$, a scanning depth of $6.5 \mathrm{~cm}$ and a corresponding in-plane image resolution of $0.12 \mathrm{~mm}$ per pixel. In order to perform segmentation, the US images are transferred at a frame rate of 30 frames-per-second to a computer $\left(2.80 \mathrm{GHz}\right.$ Intel ${ }^{\circledR}$ I7, 8-GB internal memory and 64-bit Windows 7) using an Epiphan DVI2USB 3.0 frame grabber (Epiphan Systems Inc, Ottawa, Canada).

\subsubsection{Steerable and flexible catheter (SFC) modeling}

The tendon-driven SFC used in this study is actuated by twelve tendons as depicted in Fig. 4.3. Kinematic modeling of the SFC is often described by a robot-specific and a robot-independent submapping 31,111. However, the design of the catheter steering module, which uses a single virtual tendon to control the SFC, eliminates the robot-specific submapping. Details of the virtual tendon configuration are provided in the next section (Section 4.2.3). The direct kinematics $\left(\mathbf{H}_{t}^{b 2} \in S E(3)\right)$ of the SFC is described by the following 
robot-independent submapping:

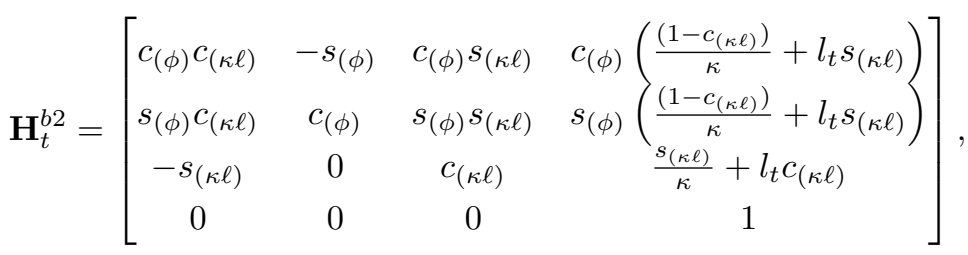

where the SFC tip frame $\left\{\Psi_{t}\right\}$ is expressed in the SFC base frame $\left\{\Psi_{b 2}\right\}$ by using the arc parameters and the SFC tip length $\left(l_{t}\right)$. The arc parameters are described by the bending direction $(\phi)$, the steerable segment curvature $(\kappa)$ and the steerable segment length $(\ell)$. Note, that the discontinuity for $\kappa=0$ in (4.1) can be overcome by substituting the differentiable cardinal sine and versine functions. Further, the steerable segment length $(\ell)$ and the SFC tip length $\left(l_{t}\right)$ are considered a constant.

The robot-specific submapping is replaced by a mapping described by using a single and virtual tendon. The proposed virtual tendon is considered to be aligned with the desired bending direction $(\phi)$ of the SFC steerable segment and is controlled by the virtual tendon displacement $(\delta)$. Hence, by solving for the virtual tendon displacement $(\delta)$, the desired SFC tip deflection can be achieved. By considering a SFC driven by three or more tendons that are equally spaced on a periphery of the instrument, the mapping described by a virtual tendon aligned with the desired SFC bending direction can be used to replace a mapping provided by a catheter specific tendon configuration. The equally spaced tendons on a periphery of the SFC are mapped in a mirrored configuration to the pivoting disc of the catheter steering module. Hence, the SFC bending direction $(\phi)$ is obtained by pivoting the disc the same direction. Subsequently, the virtual tendon displacement $(\delta)$ is obtained by a disc pivot angle $(\gamma)$, which is used to provide the desired SFC tendon configuration. Thus, the desired SFC tip deflection is obtained. Therefore, the mapping described by using a virtual tendon eliminates the use of a robot-specific submapping which is necessary to solve for the individual tendon lengths of the SFC. Thus, applicable to a wide range of tendon driven catheters. Corresponding to the virtual tendon displacement $(\delta)$, the curvature $(\kappa)$ of the SFC steerable segment is described by

$$
\kappa=\frac{\delta}{\ell d_{b}}
$$

where $d_{b}$ is virtual tendon moment arm with respect to the SFC backbone.

By considering a desired SFC tip position $\left(\mathbf{d}_{t} \in \mathbb{R}^{3}\right.$ (frame $\left.\left\{\Psi_{b 2}\right\}\right)$, where $\mathbf{d}_{t}=$ $\left[\begin{array}{lll}d_{x} & d_{y} & d_{z}\end{array}\right]^{T}$ ) and by using the direct kinematics in 4.1 , we can formulate 
the inverse kinematics according to

$$
\left[\begin{array}{c}
\mathbf{d}_{t} \\
1
\end{array}\right]=\mathbf{H}_{t}^{b 2}\left[\begin{array}{c}
\mathbf{o}_{t} \\
1
\end{array}\right]=\left[\begin{array}{c}
c_{(\phi)} \\
s_{(\phi)}\left(\begin{array}{c}
\left.\frac{\left(1-c_{(\kappa \ell)}\right)}{\kappa}+l_{t} s_{(\kappa \ell)}\right) \\
\left.\frac{\left(1-c_{(\kappa \ell)}\right)}{\kappa}+l_{t} s_{(\kappa \ell)}\right) \\
\kappa
\end{array} l_{t} c_{(\kappa \ell)}\right. \\
1
\end{array}\right]
$$

where the origin of the SFC tip frame $\left\{\Psi_{t}\right\}$ is given by $\left(\mathbf{o}_{t} \in \mathbb{R}^{3}\right.$, where $\mathbf{o}_{t}=$ $\left[\begin{array}{lll}0 & 0 & 0\end{array}\right]^{T}$ ). The SFC bending direction can be evaluated by using the desired tip position $\left(\mathbf{d}_{t}\right)$ according to

$$
\phi=\operatorname{atan} 2\left(d_{y}, d_{x}\right)
$$

which can be substituted into 4.3 in order to numerically solve for the steerable segment curvature $(\kappa)$. The evaluated bending direction $(\phi)$ and the curvature $(\kappa)$ can be used to compute the corresponding virtual tendon displacement $(\delta)$, which is provided as follows:

$$
\delta=\kappa \ell d_{b}
$$

Subsequently, the computed virtual tendon displacement $(\delta)$ can be used to evaluate the pose of the catheter steering module.

\subsubsection{Catheter steering module}

The catheter steering module is used to control a SFC independent of its tendon configuration (Fig. 4.3). A virtual tendon is considered in the bending direction of the SFC steerable segment, which can be described by $\mathbf{l}_{d}$ in the catheter steering module disc frame $\left\{\Psi_{d}\right\}$ as depicted in Fig. 4.4. The disc frame $\left\{\Psi_{d}\right\}$ orientation can be obtained by evaluating the desired SFC bending direction $(\phi)$ and the disc pivot angle $(\gamma)$, which is used to provide the desired virtual tendon displacement $(\delta)$.

A minimal configuration of two linear motors is used to control the orientation of the disc frame $\left\{\Psi_{d}\right\}$. By considering the origin of the disc frame $\left\{\Psi_{d}\right\}$ and the linear motor attachment $\left(\mathbf{p}_{m d, b}\right)$ fixed in position (frame $\left\{\Psi_{b m}\right\}$ ), a rotation about the $y$-axis (frame $\left\{\Psi_{d}\right\}$ ) can be obtained by moving the linear motor $(a)$ axis, which is used to control $\left(\mathbf{p}_{m d, a}\right)$. Similarly, rotation about the $x$-axis (frame $\left\{\Psi_{d}\right\}$ ) is provided by linear motor $(b)$. Further, a combination of linear motors $(a)$ and $(b)$ can be used to achieve SFC bending $(\phi)$ in a desired direction.

Note, that the proposed method assumes the $x$-axis of the disc frame $\left\{\Psi_{d}\right\}$ to stay in the plane described by the $x$ - and $z$-axes (frame $\left\{\Psi_{b m}\right\}$ ) as depicted in the left inset (Fig. 4.4). Similarly it is assumed that the $y$-axis of the disc frame $\left\{\Psi_{d}\right\}$ remains in the plane described by the $y$ - and $z$-axes (frame $\left.\left\{\Psi_{b m}\right\}\right)$ ). 


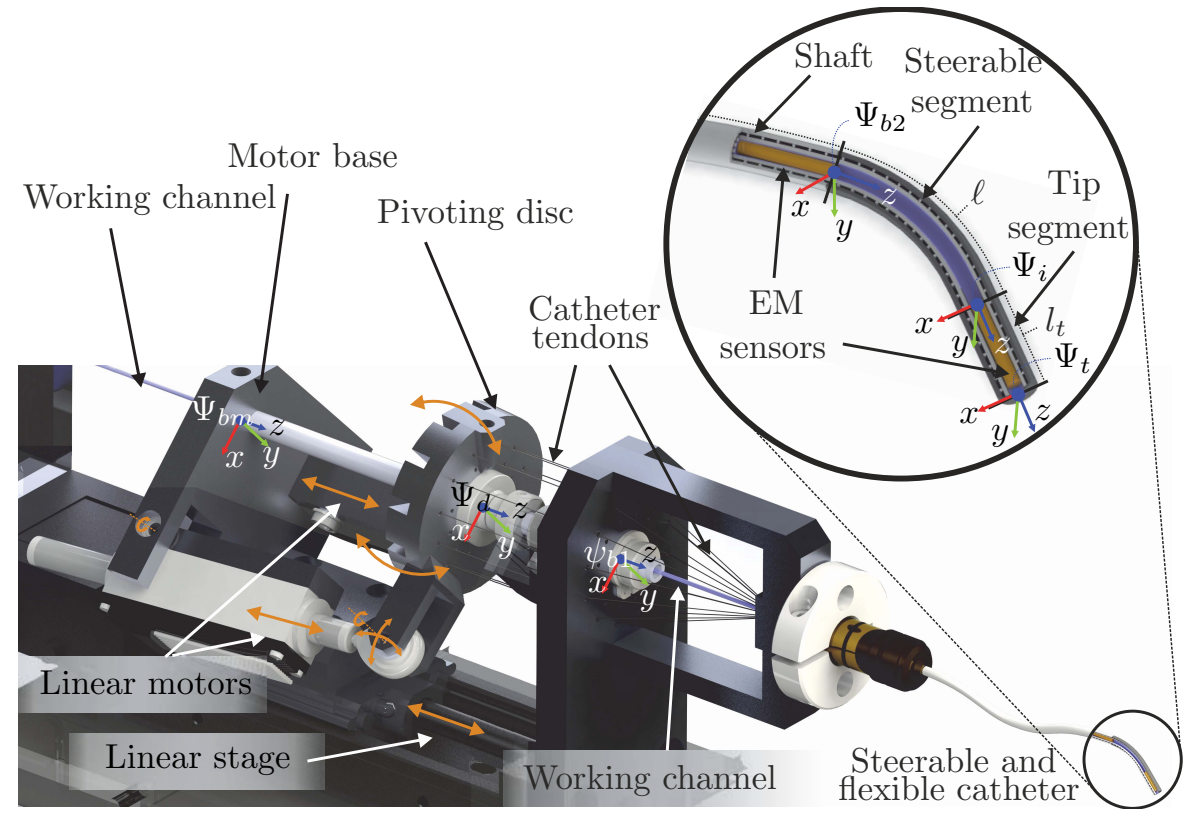

Figure 4.3: An overview of the catheter steering module used to control a steerable and flexible catheter (SFC) independent of its tendon configuration. Two linear motors are attached to the motor base frame $\left\{\psi_{b m}\right\}$, which control the orientation of the disc frame $\left\{\psi_{d}\right\}$. The hollow spherical joint of the pivoting disc is fixed in position to the hollow rod of the linear stage carriage. This prevents the pivoting disc from travelling along the longitudinal axis ( $z$-axis, frame $\left\{\psi_{b m}\right\}$ ), while the orientation can be controlled by using the linear motors. Further, the linear stage is used to provide a pretension on the tendons of the SFC. Note, that the hollow rod provides a passage for the SFC working channel. The SFC is manipulated by rotating the disc frame $\left\{\psi_{d}\right\}$ about its $x$ - or $y$-axis, which enables the articulating tip (frame $\left\{\psi_{t}\right\}$ ) to be positioned in two dimensions. The SFC is actuated by twelve tendons and comprises of a flexible shaft between base frames $\left\{\psi_{b 1}\right\}$ and $\left\{\psi_{b 2}\right\}$, a steerable segment with arc length $(\ell)$ between base frame $\left\{\psi_{b 2}\right\}$ and intermediate frame $\left\{\psi_{i}\right\}$ and a rigid link with length $\left(l_{t}\right)$, which is attached to frame $\left\{\psi_{i}\right\}$. A linear stage is used to add pretension on the tendons of the SFC, while the working channel of the SFC is equipped with two electromagnetic tracking sensor in order to provide feedback of the SFC frames $\left\{\psi_{b 2}\right\}$ and $\left\{\psi_{t}\right\}$, respectively.

However, simultaneous operation of linear motors $(a)$ and $(b)$ introduces a motion out of the aforementioned planes, which can be neglected for small pivoting angles of the disc frame (this application). For large disc pivoting angles, the rotary joints of the linear motors at their base attachments $\left(\mathbf{p}_{m b, a}\right)$ and $\left(\mathbf{p}_{m b, b}\right)$ should be relaxed in order to avoid an over-constrained system.

By considering the desired SFC tip position $\left(\mathbf{d}_{t}\right)$, the corresponding SFC bending direction $(\phi)$ and virtual tendon displacement $(\delta)$ can be computed according to (4.4) and 4.5, respectively. The SFC bending direction $(\phi)$ and virtual tendon displacement $(\delta)$ are used to compute the rotation ma- 


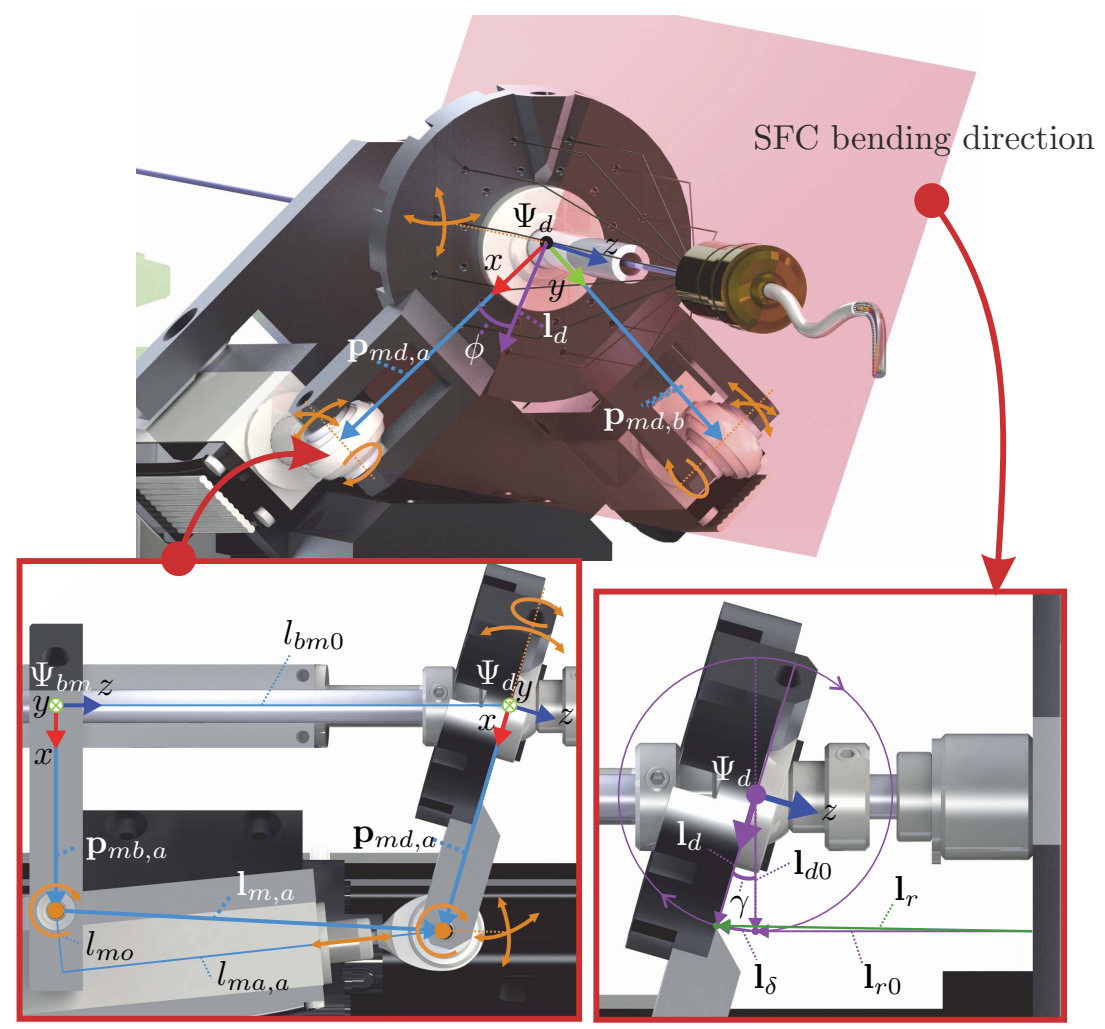

Figure 4.4: A partial view of the actual catheter steering module. Note, that all components are part of the actual catheter steering module and that for the sake of clarity, viewobstructing and irrelevant components to the control mechanism of the disc (frame $\left\{\Psi_{d}\right\}$ ) orientation are omitted from view. Further, joint orientations and translations are shown in orange. Two linear motors (a) and (b) are independently controlled and attached to the disc in order to enable rotation about the $y$ - and $x$-axes (frame $\left\{\Psi_{d}\right\}$ ), respectively. The right inset shows a cross section (red plane) of the catheter steering module in the SFC bending direction $(\phi)$. Note, that rotating the disc in the SFC bending direction $(\phi)$ with pivot angle $\gamma$ can be achieved by positioning motors $(a)$ and $(b)$. Further, the left inset demonstrates the rotation about the $y$-axis of the disc frame $\left\{\Psi_{d}\right\}$. The linear motor $(a)$ of the catheter steering module is attached to the motor base using a rotary joint $\left(\mathbf{p}_{m b, a}\right.$, in frame $\left.\left\{\Psi_{b m}\right\}\right)$, while its linear axis is connected to the disc using a spherical joint $\left(\mathbf{p}_{m d, a}\right.$, in frame $\left.\left\{\Psi_{d}\right\}\right)$. Note, that the spherical joints of linear motor axes facilitates orientation of the disc about the $x$ - and $y$-axis frame $\left\{\Psi_{d}\right\}$. The desired motor configuration is given by $\mathbf{l}_{m, a}$, while the corresponding linear motor axis has a length $\left(l_{m a, a}\right)$ and is fixed with an offset $\left(l_{m o}\right)$. Further, the distance between rotating disc frame $\left\{\Psi_{d}\right\}$ and motor base frame $\left\{\Psi_{b m}\right\}$ is indicated by $l_{b m 0}$. The virtual tendon (depicted as a green arrow) and disc orientation in the desired catheter bending direction are given by $\mathbf{l}_{r}$ and $\mathbf{l}_{d}$, respectively. The corresponding initial virtual tendon and disc configurations are given by $\mathbf{l}_{r 0}$ and $\mathbf{l}_{d 0}$, while the displacement vector of the virtual tendon is denoted by $\mathbf{l}_{\delta}$ and the corresponding pivot angle is indicated by $\gamma$. 
trix $\left(\mathbf{R}_{d}^{b m} \in S O(3)\right)$ to provide the orientation of the disc frame $\left\{\Psi_{d}\right\}$ relative to the motor base frame $\left\{\Psi_{b m}\right\}$ as follows:

$$
\mathbf{R}_{d}^{b m}(\phi, \gamma)=\mathbf{R}_{z}(\phi) \mathbf{R}_{x}(\gamma) \mathbf{R}_{z}(-\phi),
$$

where we first rotation about the $z$-axis (frame $\left\{\Psi_{d}\right\}$ ) in order to enable SFC tip deflection in the desired SFC bending direction $(\phi)$. Subsequently, we rotation about the new $x$-axis (frame $\left\{\Psi_{d}\right\}$ ) by using pivot angle $(\gamma)$, which is used to achieve the desired SFC tip deflection. Finally, the original disc orientation about the $z$-axis (frame $\left\{\Psi_{d}\right\}$ ) is restored by using angle $(-\phi)$. The disc frame rotation matrix $\left(\mathbf{R}_{d}^{b m}\right)$ is given by

$$
\mathbf{R}_{d}^{b m}=\left[\begin{array}{ccc}
c_{(\phi)}^{2}+s_{(\phi)}^{2} c_{(\gamma)} & s_{(2 \phi)} s_{(\gamma / 2)}^{2} & s_{(\phi)} s_{(\gamma)} \\
s_{(2 \phi)} s_{(\gamma / 2)}^{2} & c_{(\phi)}^{2} c_{(\gamma)}+s_{(\phi)}^{2} & -c_{(\phi)} s_{(\gamma)} \\
-s_{(\phi)} s_{(\gamma)} & c_{(\phi)} s_{(\gamma)} & c_{(\gamma)}
\end{array}\right] .
$$

In order to compute the rotation matrix $\left(\mathbf{R}_{d}^{b m}\right)$ described in 4.7 , we need to evaluate the pivot angle $(\gamma)$ of the disc frame $\left\{\Psi_{d}\right\}$. By considering the virtual tendon $\left(\mathbf{l}_{r} \in \mathbb{R}^{2}\right)$, which is in the SFC bending direction $(\phi)$ as depicted in Fig. 4.4, we can formulate the desired virtual tendon displacement $(\delta)$ according to

$$
\delta(\gamma)=\left|\mathbf{l}_{r}(\gamma)\right|-\left|\mathbf{l}_{r 0}\right|,
$$

where $\mathbf{l}_{r 0} \in \mathbb{R}^{2}$ is the initial virtual tendon configuration with an offset $\left(l_{d b}\right)$ described by $\mathbf{l}_{r 0}=\left[\begin{array}{ll}0 & -l_{d b}\end{array}\right]^{T}$. We can find an expression for the desired virtual tendon configuration by

$$
\mathbf{l}_{r}(\gamma)=\mathbf{l}_{r 0}+\mathbf{l}_{\delta}(\gamma)
$$

while the initial virtual tendon configuration with offset $\left(l_{d b}\right)$ is described by $\mathbf{l}_{r 0} \in \mathbb{R}^{2}$, where $\mathbf{l}_{r 0}=\left[\begin{array}{ll}0 & -l_{d b}\end{array}\right]^{T}$. Further, the virtual tendon displacement vector is described by $\mathbf{l}_{\delta} \in \mathbb{R}^{2}$, which is given according to

$$
\mathbf{l}_{\delta}(\gamma)=\mathbf{l}_{d}(\gamma)-\mathbf{l}_{d 0}=r\left[\begin{array}{c}
c_{\left(\gamma_{y}\right)}-1 \\
-s_{\left(\gamma_{y}\right)}
\end{array}\right],
$$

where $\mathbf{l}_{d 0} \in \mathbb{R}^{2}$ describes the initial disc configuration and $\mathbf{l}_{d} \in \mathbb{R}^{2}$ indicates the orientation of the disc. By considering a desired SFC tip position, 4.8, 4.9 and $(4.10)$, the corresponding virtual tendon displacement $(\delta)$ can be evaluated according to

$$
\delta(\gamma)=\sqrt{2 r\left(l_{d b} s_{(\gamma)}-r c_{(\gamma)}\right)+2 r^{2}+l_{d b}^{2}}-l_{d b},
$$

which can be used to find an expression for the pivot angle $(\gamma)$ of the disc frame $\left\{\Psi_{d}\right\}$ by rearranging 4.11 according to

$$
\gamma=\sin ^{-1}\left(\frac{\left(\delta+l_{d b}\right)^{2}-2 r^{2}-l_{d b}^{2}}{2 r \sqrt{r^{2}+l_{d b}^{2}}}\right)-\operatorname{atan} 2\left(-r, l_{d b}\right) .
$$


Chapter 4. Sensor-Guided Stabilization of a Steerable and Flexible Catheter Tip for Beating Heart Mitral Valve Motions

By considering the desired SFC tip position $\left(\mathbf{d}_{t}\right)$, the SFC bending direction $(\phi)$ in (4.4) and the pivot angle $(\gamma)$ in 4.12 are used to compute the corresponding disc frame orientation, which is described in 4.7).

In order to obtain the desired disc frame $\left\{\Psi_{d}\right\}$ orientation, the corresponding linear motor positions must be computed. The coordinate transformation between the disc frame $\left\{\Psi_{d}\right\}$ and motor base frame $\left\{\Psi_{b m}\right\}$ is given by transformation matrix $\left(\mathbf{H}_{d}^{b m} \in S E(3)\right)$

$$
\mathbf{H}_{d}^{b m}=\left[\begin{array}{cc} 
& 0 \\
\mathbf{R}_{d}^{b m} & 0 \\
& -l_{b m 0} \\
\mathbf{0}_{1 \times 3} & 1
\end{array}\right],
$$

where $\mathbf{0}$, represents a row vector filled with zeros and $l_{b m 0}$ is the distance between frames $\left\{\Psi_{b m}\right\}$ and $\left\{\Psi_{d}\right\}$ along the $z$-axis of the motor base frame $\left\{\Psi_{b m}\right\}$. Further, the vectors $\left(\mathbf{l}_{m, a} \in \mathbb{R}^{3}\right)$ and $\left(\mathbf{l}_{m, b} \in \mathbb{R}^{3}\right)$ between linear motor attachments $\left(\mathbf{p}_{m b, a} \in \mathbb{R}^{4}\right)$ and $\left(\mathbf{p}_{m b, b} \in \mathbb{R}^{4}\right)$ of the base frame $\left\{\Psi_{b m}\right\}$ and $\left(\mathbf{p}_{m d, a} \in\right.$ $\left.\mathbb{R}^{4}\right)$ and $\left(\mathbf{p}_{m d, b} \in \mathbb{R}^{4}\right)$ of the disc frame $\left\{\Psi_{d}\right\}$ as depicted in Fig. 4.4 . can be evaluated by

$$
\left[\begin{array}{c}
\mathbf{l}_{m, *} \\
1
\end{array}\right]=\mathbf{p}_{m b, *}-\mathbf{H}_{d}^{b m} \mathbf{p}_{m d, *},
$$

where ${ }^{*}$ indicates the corresponding linear motor $(a)$ or $(b)$. The motor attachments of frame $\left\{\Psi_{b m}\right\}$ are given by $\left(\mathbf{p}_{m b, a}=\left[\begin{array}{llll}l_{m b} & 0 & 0 & 1\end{array}\right]^{T}\right)$ and $\left(\mathbf{p}_{m b, b}=\right.$ $\left.\left[\begin{array}{llll}0 & l_{m b} & 0 & 1\end{array}\right]^{T}\right)$ with offset $\left(l_{m b}\right)$, while the motor attachments of frame $\left\{\Psi_{d}\right\}$ are described by $\left(\mathbf{p}_{m d, a}=\left[\begin{array}{llll}l_{m d} & 0 & 0 & 1\end{array}\right]^{T}\right)$ and $\left(\mathbf{p}_{m d, b}=\left[\begin{array}{llll}0 & l_{m d} & 0 & 1\end{array}\right]^{T}\right)$ with offset $\left(l_{m d}\right)$. Note, that the linear motor axes have an offset $\left(l_{m o}\right)$ as depicted in Fig. 4.4. Hence, the corresponding linear motor axis length can be computed according to

$$
l_{m a, *}=\sqrt{\left|\mathbf{l}_{m, *}\right|^{2}-l_{m 0}^{2}}
$$

By applying the linear motor axis length in 4.15), we obtain the desired disc frame $\left\{\Psi_{d}\right\}$ orientation $\left(\mathbf{R}_{d}^{b m}\right)$. Thus, we achieve the desired SFC tip position.

\subsubsection{Steerable and flexible catheter tracking and seg- mentation}

In order to provide feedback of the SFC tip and the MV motion embedded in the experimental setup, we use EM tracking sensors and 2D US segmentation as depicted in Fig. 4.5. The EM tracking sensors are integrated in the working channel of the SFC in order to provide instrument feedback for closed-loop control. Further, 2D US imaging is used to obtain feedback of the SFC tip and the beating heart MV motions, which is used for instrument tip stabilization. 


\subsubsection{Electro-Magnetic tracking}

A 5DOF EM sensor is embedded in the working channel of the SFC and located at the base frame $\left\{\Psi_{b 2}\right\}$, which provides feedback on the position along the $x$ , $y$ - and $z$-axes of frame $\left\{\Psi_{b 2}\right\}$ and the orientation about the $x$ - and $y$-axes of frame $\left\{\Psi_{b 2}\right\}$. Further, a 6 DOF EM sensor is integrated in the working channel of the SFC, which is at the tip frame $\left\{\Psi_{t}\right\}$ and provides position and orientation feedback. The 6DOF EM sensor at the SFC tip frame $\left\{\Psi_{t}\right\}$ comprises of two collinear 5DOF EM sensors with a fixed distance, which is used to find the orientation about the $z$-axis of frame $\left\{\Psi_{t}\right\}$. Further, the fixed and collinear configuration of the two 5DOF EM sensors at the tip provides measurement redundancy, which can be used to estimate sensor disturbances.

\subsubsection{Ultrasound segmentation}

US segmentation techniques are used to evaluate the beating heart MV motions and the SFC tip position in 2D. A radial cross-sectional view of the SFC within the annulus of the MV is provided in Fig. 4.5. Note, that in this study we assume alignment of the US image plane with the MV annulus by using a fixed US transducer position. In order to enhance the visibility of the instrument, we increase the acoustic impedance difference between the SFC tip and its surround materials. The acoustic impedance $(z)$ of a material is given by $z=$ $\rho c$, where $\rho$ denotes the density and $c$ the acoustic velocity of the material. By improving the reflection coefficient $(r)$, which describes the acoustic impedance difference at an interface between two materials according to

$$
r=\left(\frac{\rho_{2} c_{2}-\rho_{1} c_{1}}{\rho_{2} c_{2}+\rho_{1} c_{1}}\right)^{2},
$$

we can enhance the contrast between the SFC tip and its surrounding materials 140 . Hence, by considering a thin film of copper $\left(\rho_{2}=8960 \mathrm{~kg} / \mathrm{m}^{3}\right.$ and $\left.c_{2}=3810 \mathrm{~m} / \mathrm{s}\right)$ covering the SFC tip and the surrounding materials such as water $\left(\rho_{1}=988 \mathrm{~kg} / \mathrm{m}^{3}\right.$ and $\left.c_{1}=1497 \mathrm{~m} / \mathrm{s}\right)$ or blood $\left(\rho_{b}=1025 \mathrm{~kg} / \mathrm{m}^{3}\right.$ and $c_{b}=1570 \mathrm{~m} / \mathrm{s}$ ), we obtain a reflection coefficient $(r)$ of approximately $84 \%$. Note, that in the clinical setting, the bio-incompatible material copper can be replaced by gold $\left(\rho_{b}=19290 \mathrm{~kg} / \mathrm{m}^{3}\right.$ and $\left.c_{b}=3240 \mathrm{~m} / \mathrm{s}\right)$.

The SFC tip is segmented in 2D US images as depicted in the inset of Fig. 4.5, which shows the radial cross-sectional view of the instrument. We evaluate the reflected semi-circular shape of the SFC tip, which describes the instrument surface. We apply preprocessing such as Gaussian filtering to reduce speckle and noise in US images. Further, by applying a Canny edge detector with a hysteresis threshold, we can obtain an edge map op the semi-circular surface of the SFC tip. A hysteresis threshold reduces the sensitivity to variations in contrast of the US image, which is often caused by differences in acoustic impedances between the SFC tip and the surrounding materials. Subsequently, 


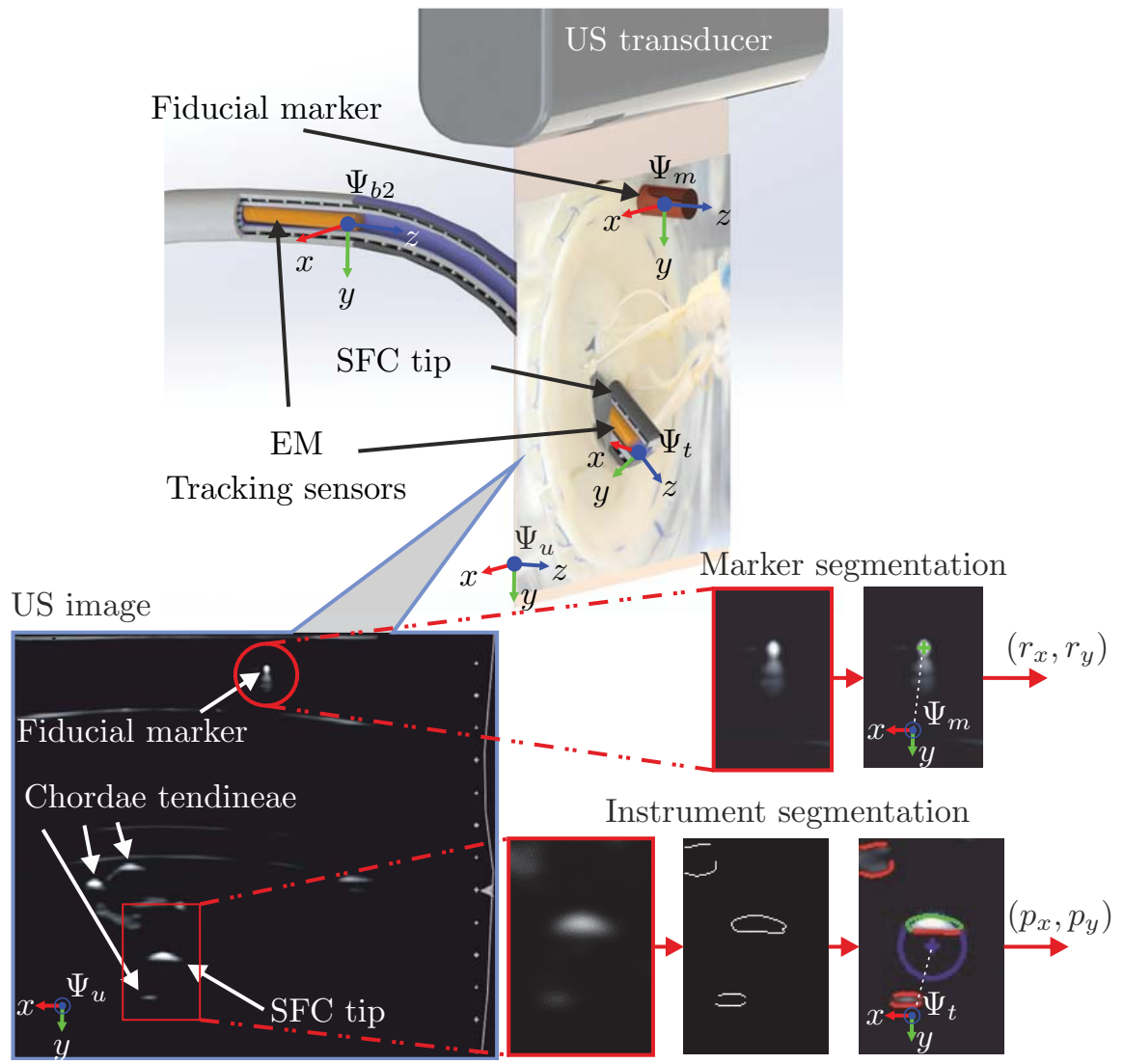

Figure 4.5: An overview of electromagnetic (EM) tracking and two-dimensional ultrasound (US) segmentation of the steerable and flexible catheter (SFC) tip and the mitral valve (MV) pose. An EM sensor is located at the SFC base frame $\left\{\Psi_{b 2}\right\}$, while an second EM sensor is positioned at the SFC tip frame $\left\{\Psi_{t}\right\}$. The US transducer is oriented along the MV annular plane with corresponding US image frame $\left\{\Psi_{u}\right\}$, which is depicted in the bottomleft inset, where a radial cross-sectional view of the SFC tip and the MV fiducial marker is shown. In order to obtain the beating heart MV motions, a fiducial marker (frame $\left\{\Psi_{m}\right\}$ ) is attached to the MV, which is segmented in US images. The centroid of the fiducial marker is segmented (top-right insets) using a blob detection algorithm, which is evaluated as the green cross and provides reference signals $\left(r_{x}\right.$ and $\left.r_{y}\right)$ for closed-loop control. Further, the centroid of the SFC tip $\left(p_{x}, p_{y}\right)$ is evaluated by segmentation techniques (bottom-right insets) such as Gaussian filtering, Canny edge detection and a random sample consensus algorithm, where inliers (green) and outlier (red) describe the semi-circular surface of the SFC tip. Note, that soft tissue such as the chordae tendineae, which is evaluated as part of the edge map, is considered to be an outlier (red) to the semi-circular surface of the SFC tip.

we use a random sample consensus algorithm (RANSAC) to evaluate the SFC tip center position $\left(p_{x}, p_{y}\right)$. The RANSAC algorithm uses the parametric description of a semi-circle to evaluate the SFC tip in US images. Robustness is 
provided by the RANSAC algorithm, which excludes irrelevant edges that do not conform with the parametric description of the SFC tip [110.

In order to evaluate the beating heart MV motions, we attach a fiducial marker to the MV model. The fiducial marker made of copper is extracted by using a blob detection algorithm. Note, that copper marker used in this study can be replaced by a gold fiducial marker, which is often used in clinical practice 141]. A blob classifies itself by a circular region in an image with a different contrast than the surrounding regions. The blob that represents the fiducial marker is segmented by thresholding, where the resulting crosssectional area is evaluated in size and circularity. The evaluated center $\left(r_{x}, r_{y}\right)$ of the blob is used as the fiducial marker position in closed-loop control.

In order to enable coordinate transformations used to express the frames of the experimental setup into a common coordinate system, we evaluate the EM sensor data and the results of US image segmentation. The coordinate transformation $\left(\mathbf{H}_{b 2}^{u} \in S E(3)\right)$ is used to describe the coordinates of the SFC base frame $\left\{\Psi_{b 2}\right\}$ in the the static ultrasound image frame $\left\{\Psi_{u}\right\}$, which is provided by the $5 \mathrm{DOF}$ EM sensor at the SFC base (frame $\left\{\Psi_{b 2}\right\}$ ). Note, that by assuming torsional stiffness of the SFC steerable segment, we can obtain the orientation about the $z$-axis of the SFC base frame $\left\{\Psi_{b 2}\right\}$ by using the 6 DOF EM sensor at the SFC tip frame $\left\{\Psi_{t}\right\}$. Further, the coordinate transformation $\left(\mathbf{H}_{t}^{u} \in S E(3)\right)$, which is enabled by segmentation, expresses the SFC tip (frame $\left\{\Psi_{t}\right\}$ ) in US images (frame $\left\{\Psi_{u}\right\}$ ). Similarly, segmentation is used to describe the fiducial marker frame $\left\{\Psi_{m}\right\}$ in US images (frame $\left\{\Psi_{u}\right\}$ ), which is given by coordinate transformation $\left(\mathbf{H}_{m}^{u} \in S E(3)\right)$. By using the EM sensors and results of US segmentation, the coordinate transformations can be used to express the SFC frames and the beating heart MV motions in a common coordinate system, which enables closed-loop control.

\subsubsection{Sensor-guided controller}

The control strategy comprises of two independently steered systems as depicted in Fig. 4.6. The first system (top, Fig. 4.6), a Stewart platform is used to reproduce beating heart MV motions along the plane of the MV annulus ( $x$ and $y$-axes, frame $\left\{\Psi_{u}\right\}$ ), while a pulsatile pump is used to enable opening and closing of the MV. The second system (bottom, Fig. 4.6), stabilizes the SFC tip for the beating heart MV motions.

The beating heart MV motions $\left(i_{x}\right.$ and $\left.i_{y}\right)$ along $x$ - and $y$-axes of frame $\left\{\Psi_{u}\right\}$ are reproduced using a $6 \mathrm{DOF}$ Stewart platform, which is actuated by joint angels $\left(\boldsymbol{\theta} \in \mathbb{R}^{6}\right.$, where $\left.\boldsymbol{\theta}=\left[\begin{array}{lll}\theta_{1} & \ldots & \theta_{6}\end{array}\right]^{T}\right)$. The beating heart MV motions are provided by pre-operative patient data, which we describe in Section 4.2.6. By considering the MV motions $\left(i_{x}\right.$ and $\left.i_{y}\right)$ and the inverse kinematics of the Stewart platform, we can obtain the joint angles $(\boldsymbol{\theta})$ of the Stewart platform 142]. The aforementioned system functions independently of the SFC closed-loop controller. Hence, by US segmentation of the MV fiducial marker, we provide 

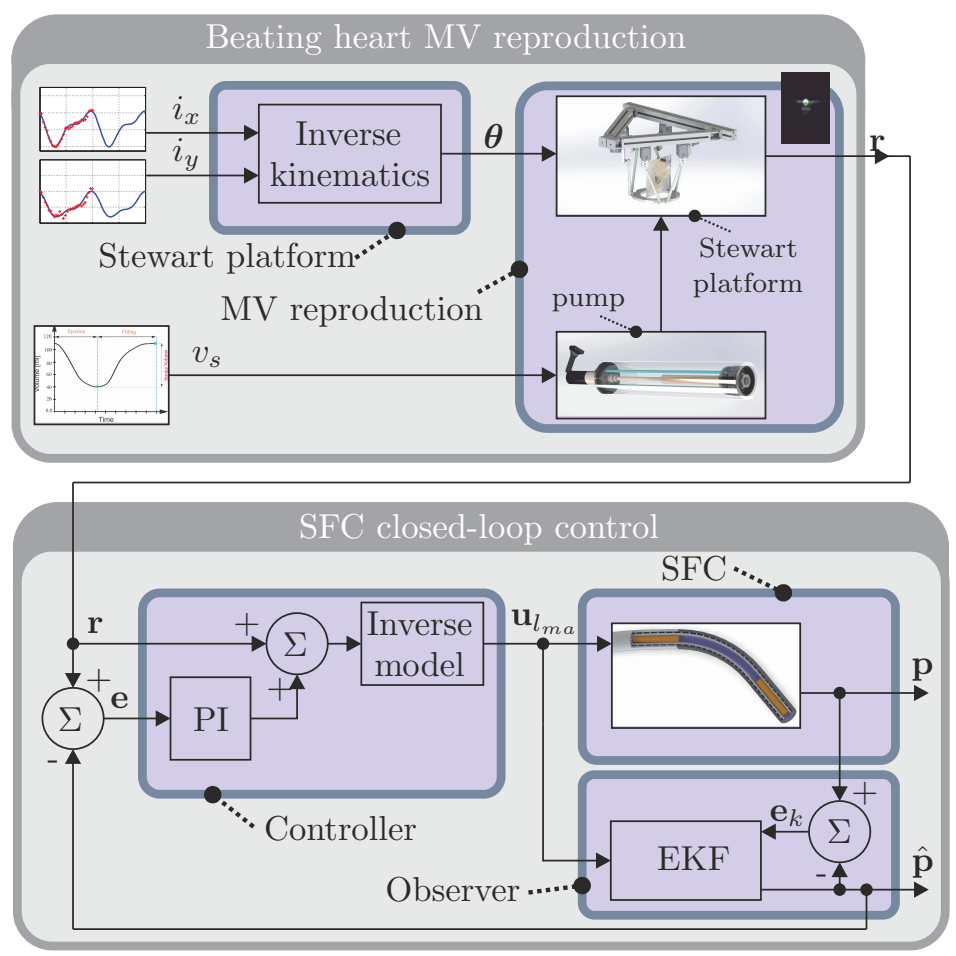

Figure 4.6: An overview of the independently-controlled systems: Beating heart mitral valve (MV) reproduction and the closed-loop control strategy of the steerable and flexible catheter (SFC) tip. Closed-loop control is used to stabilize the SFC tip for the reproduced beating heart MV motions. A Stewart platform with joint angles $(\boldsymbol{\theta})$ is used to reproduce beating heart MV motions based on pre-operative patient data $\left(i_{x}\right.$ and $\left.i_{y}\right)$, while a pulsatile pump provides the desired stroke volume $\left(v_{s}\right)$ to enable opening and closing of the MV. Ultrasound segmentation is used to evaluate the MV motion, which is provided as a reference $\left(\mathbf{r}=\left[\begin{array}{ll}r_{x} & r_{y}\end{array}\right]^{T}\right)$ to closed-loop control of the SFC tip. The control signal $\left(\mathbf{u}_{l_{m a}}=\left[l_{m a, x} l_{m a, y}\right]^{T}\right)$ obtained by inverse kinematics of the SFC and catheter steering module is used to manipulate the linear motors lengths $\left(l_{m a, x}\right.$ and $\left.l_{m a, y}\right)$ of the catheter steering module, which results in the desired SFC tip position $\left(\mathbf{p}=\left[p_{x} p_{y}\right]^{T}\right)$. Further, ultrasound segmentation and electromagnetic tracking is used to evaluate the SFC tip position $(\mathbf{p})$, while its filtered version $\left(\hat{\mathbf{p}}=\left[\begin{array}{ll}\hat{p_{x}} & \hat{p_{y}}\end{array}\right]^{T}\right)$ is provided by an extended kalman filter (EKF) updated by error signal $\left(\mathbf{e}_{k}=\mathbf{p}-\hat{\mathbf{p}}\right)$. A proportional and integral (PI) control action is used to address the reference tracking error $(\mathbf{e}=\mathbf{r}-\hat{\mathbf{p}})$.

a reference signal $\left(\mathbf{r} \in \mathbb{R}^{2}\right.$, where $\left.\mathbf{r}=\left[r_{x} r_{y}\right]^{T}\right)$, which can be used as an input to the SFC closed-loop controller.

The SFC closed-loop controller is used to stabilize the SFC tip for the beating heart MV motions. The reference signal (r) is evaluated by US segmentation and used to compute the control signal $\left(\mathbf{u}_{l_{m a}} \in \mathbb{R}^{2}\right.$, where $\mathbf{u}_{l_{m a}}=$ $\left.\left[\begin{array}{ll}l_{m a, x} & l_{m a, y}\end{array}\right]^{T}\right)$. The signal $\left(\mathbf{u}_{l_{m a}}\right)$ controls the catheter steering module mo- 
tor lengths $\left(l_{m a, x}\right.$ and $\left.l_{m a, y}\right)$ described in 4.15 by using inverse models as follows: First, the reference signal (r) is used to solve the SFC inverse kinematics described in (4.3), which yields the desired virtual tendon displacement in 4.8. . Note, that the SFC tip motion perpendicular to the MV annular plane (longitudinal axis of the SFC), which is caused by the SFC bending, is not addressed in this study. Subsequently, we use the desired virtual tendon displacement to compute the disc frame $\left\{\Psi_{d}\right\}$ pose as described in 4.13 . Finally, the corresponding linear motor lengths described in 4.15 is obtained by the disc frame $\left\{\Psi_{d}\right\}$ pose and 4.14). Feedback of the SFC tip position $\left(\mathbf{p} \in \mathbb{R}^{2}\right.$, where $\left.\mathbf{p}=\left[\begin{array}{ll}p_{x} & p_{y}\end{array}\right]^{T}\right)$ is provided by an EM sensor integrated in the tip and by US image segmentation. We use an extended Kalman filter (EKF) in order to reduce noise and to provide the estimated SFC tip position $\left(\hat{\mathbf{p}} \in \mathbb{R}^{2}\right.$, where $\hat{\mathbf{p}}=\left[\hat{p_{x}} \hat{p_{y}}\right]^{T}$ ). Feedback is used to close the control loop by evaluating the MV reference tracking error $\left(\mathbf{e} \in \mathbb{R}^{2}\right)$, which is the result of modeling mismatch and disturbances acting on the system. The complete closed-loop control system operates at $25 \mathrm{~Hz}$, which is limited by the ultrasound image segmentation of the fiducial marker and the SFC tip positions. The reference tracking error (e) given by $\mathbf{e}=\mathbf{r}-\hat{\mathbf{p}}$ is reduced by using a proportional and integral (PI) control action with empirically evaluated gains. Therefore, stabilization of the SFC tip for the beating heart MV motions is provided.

\subsubsection{Beating heart mitral valve modeling}

An overview of MV motion modeling is shown in Fig. 4.7. A realistic and functional MV is embedded in an acrylic heart model attached to a Stewart platform, which is used to reproduce beating heart MV motions. Further, a piston driven pulsatile pump provides the desired stroke volume $\left(v_{s}\right)$ of approximately $50 \mathrm{ml}$ that enables the MV to open and close during the cardiac cycle. In this study, we used pre-operative patient data to describe the MV motions at a rate of 30 and 9 volumes-per-second, which is obtained by a 1.5 Tesla MR imaging scanner and a TEE system (Philips Healthcare, Amsterdam, the Netherlands), respectively. Manual segmentation of seven (MR data) and twelve (US data) cross-sectional images provides the MV annulus. In order to find the pose of the MV annular plane during the cardiac cycle, we use principle component analysis (PCA) according to: First, the plane of the MV annulus is provided by the two eigenvectors of the PCA covariance matrix with the largest variances. Subsequently, the eigenvector with the least amount of variance, which is normal to the MV annular plane, is used to complete the orientation of the MV annulus 143,144. The periodic MV motion is described by a using two-term Fourier series according to

$$
i_{x}=a_{0 x}+a_{1_{x}} c_{\frac{k}{S_{r}}} \omega+b_{1_{x}} s_{\frac{k}{S_{r}} \omega}+a_{2 x} c_{2 \frac{k}{S_{r}} \omega}+b_{2 x} s_{2 \frac{k}{S_{r}} \omega},
$$

and

$$
i_{y}=a_{0 y}+a_{1 y} c_{\frac{k}{S_{r}}} \omega+b_{1_{y}} s_{\frac{k}{S_{r}} \omega}+a_{2 y} c_{2 \frac{k}{S_{r}} \omega}+b_{2 y} s_{2 \frac{k}{S_{r}}} \omega
$$


Chapter 4. Sensor-Guided Stabilization of a Steerable and Flexible Catheter Tip for Beating Heart Mitral Valve Motions

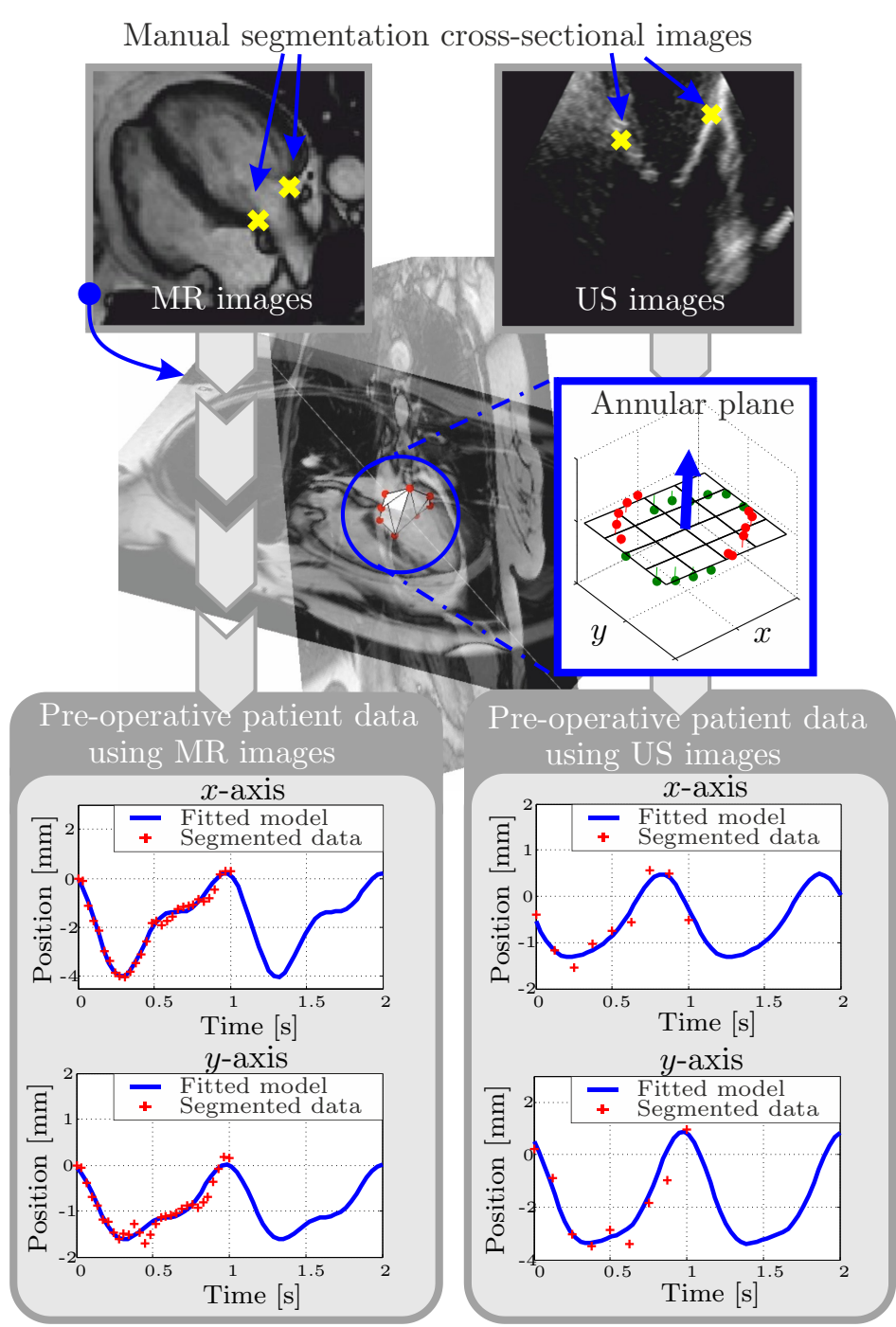

Figure 4.7: An overview of mitral valve (MV) motion modeling obtained from pre-operative patient data. Manual segmentation of cross-sectional magnetic resonance (MR) (top-left inset) and ultrasound (US) (top-right inset) images provides the MV annulus as depicted in the center inset. By evaluating the annular plane position during the cardiac cycle, we obtain the MV motions along the $x$ - and $y$-axes (frame $\left\{\Psi_{u}\right\}$ ) for MR and US pre-operative patient data as depicted in bottom-left and bottom-right graphs, respectively.

where $k$ denotes discrete time, $S_{r}$ the sample rate, while the frequency $(\omega)$ of the periodic MV motion is given by $\omega=2 \pi$ and the coefficients are provided in Table 4.1. The periodic signals $\left(i_{x}\right.$ and $\left.i_{y}\right)$ described in 4.17) and 4.18 are 
Table 4.1: Table of Fourier coefficients used in 4.17 and 4.18 in order to described the periodic mitral valve motions obtained form pre-operative patient data using magnetic resonance (MR) and ultrasound (US) images. The subscript ${ }^{*}$ denotes the $x$ - or $y$-axis (frame $\left\{\Psi_{u}\right\}$ ) with corresponding goodness of fit provided by the square of the correlation $\left(R^{2}\right)$.

\begin{tabular}{llllllll}
\hline & axis & $a_{0_{*}}$ & $a_{1_{*}}$ & $b_{1_{*}}$ & $a_{2_{*}}$ & $b_{2_{*}}$ & $R^{2}$ \\
\hline \multirow{2}{*}{ US } & $x$ & -0.550 & 0.120 & -0.871 & -0.116 & -0.115 & 0.87 \\
& $y$ & -1.700 & 1.813 & -0.985 & 0.410 & -0.321 & 0.96 \\
\hline \multirow{2}{*}{ MR } & $x$ & -1.735 & 0.888 & -1.413 & 0.804 & -0.006 & 0.98 \\
& $y$ & -0.906 & 0.584 & -0.363 & 0.273 & -0.050 & 0.93 \\
\hline
\end{tabular}

provided as an input to the Stewart platform to reproduce the beating heart MV motions from MR and US pre-operative patient data, which is used to experimentally evaluate SFC tip stabilization.

\subsection{Experiments}

In this section, we described the experiments performed in order to evaluate sensor-guided control of the SFC tip. Subsequently, we present the corresponding accuracy results of SFC tip stabilization for the beating heart MV motions.

\subsubsection{Experimental plan}

Experiments are performed in order to evaluate the performance of the presented SFC tip stabilization methods for beating heart MV motions. The SFC tip is inserted in the realistic and functional MV model embedded in the acrylic heart system. In experiments, the Stewart platform is used to reproduce the beating heart MV motions paths based on pre-operative patient data as depicted in Fig. 4.7, while a pulsatile pump provides fluid flow to enable opening and closing of the realistic MV valve. The results of US fiducial marker segmentation is considered as a reference (r) for SFC closed-loop control. In experimental scenarios using multiple SFC tip feedback modalities, we evaluate SFC tip stabilization for beating heart MV motions: First, by using electromagnetic SFC tip tracking. Second, by considering ultrasound SFC tip segmentation. We reproduce the beating heart MV motions during experiments for heart rates of 10, 20 and $30 \mathrm{BPM}$. The aforementioned heart rates can be achieved by considering the frequency $(\omega)$ in 4.17) and 4.18, which describes the periodic MV motion. Note, that the motion compensation experiments are limited to heart rates up to 30 BPM. This limitation is caused by ultrasound detection of the fiducial marker, which is considered as a reference signal in closed-loop control. 
Chapter 4. Sensor-Guided Stabilization of a Steerable and Flexible Catheter Tip for Beating Heart Mitral Valve Motions

\subsubsection{Results}

The results of the experiments described in the experimental plan are reported in Table 4.2. A representative of EM- and US-guided SFC tip stabilization for beating heart MV motions based on MR (20 BPM) and US (30 BPM) preoperative patient data are shown in Fig. 4.8. The SFC tip stabilization experiments are repeated 5 times, which indicated a repeatably less than $\pm 0.1 \mathrm{~mm}$.

Experiments show a deterioration in SFC tip stabilization accuracy for an increased heart rate up to $30 \mathrm{BPM}$. This could be the delayed observations of the MV marker, which is considered to be significant for faster heart rates. Experiments with a reproduced heart rate of 30 BPM show a mean absolute distance error $(\epsilon)$ of $0.57 \mathrm{~mm}$ for EM-guided stabilization, while US-guided stabilization showed a mean absolute distance error $(\epsilon)$ of $1.39 \mathrm{~mm}$. The increased error of US-guided stabilization could be attributed to the delay of approximately 200 milliseconds, which is present in the ultrasound feedback. For experiments with a reproduced heart rate of $10 \mathrm{BPM}$, we observe mean absolute distance errors $(\epsilon)$ of $0.42 \mathrm{~mm}$ and $0.53 \mathrm{~mm}$ for EM- and US-guided SFC tip stabilization, respectively. The aforementioned results show a minor difference and can be considered insignificant. Further, we observe an increased

Table 4.2: Experimental results of the steerable and flexible catheter (SFC) tip stabilization for the beating heart mitral valve motions based on pre-operative patient data obtained from magnetic resonance (MR) and ultrasound (US) images. The results of the electromagneticand ultrasound-guided controllers for heart rates of 10, 20 and 30 beats-per-minute (BPM) are provided. The mean absolute distance error $(\epsilon)$ and the mean absolute position errors $\left(\epsilon_{x}\right.$ and $\left.\epsilon_{y}\right)$ along the $x$ - and $y$-axes are presented with the standard deviation of the absolute error.

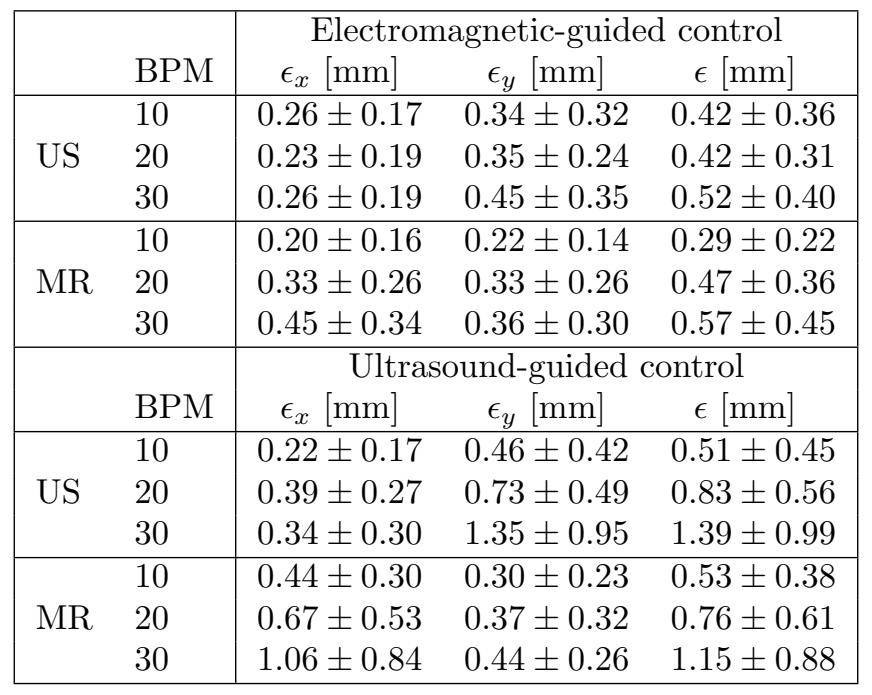




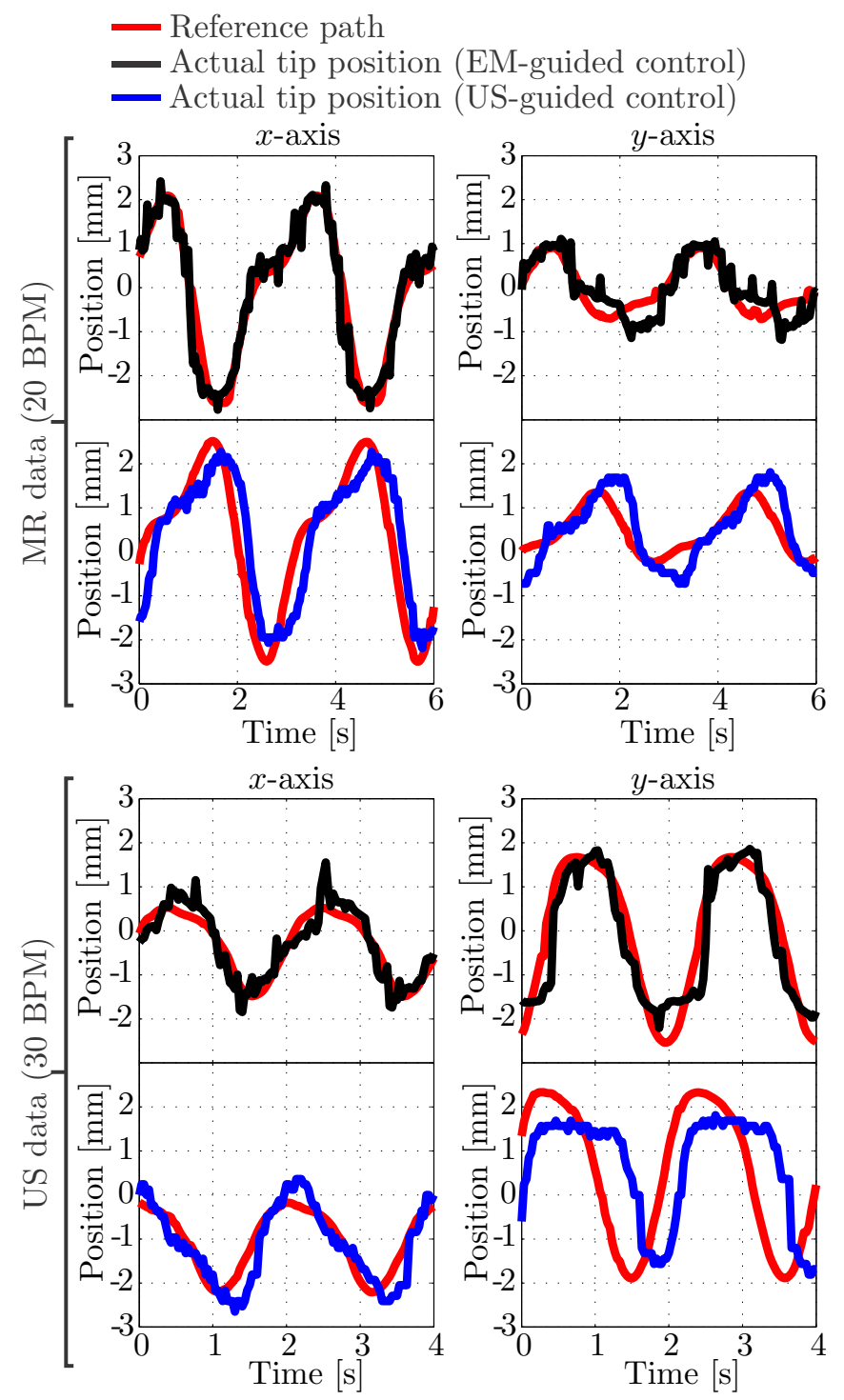

Figure 4.8: Representative experimental sensor-guided stabilization results of the steerable and flexible catheter (SFC) articulating tip for beating heart mitral valve (MV) motions based on magnetic resonance (MR) and ultrasound (US) pre-operative patient data for heart rates of 20 and 30 BPM. The red line describes the reference path $\left(\mathbf{r}=\left[r_{x} r_{y}\right]^{T}\right)$ obtained from marker segmentation described in Section 4.2.4 while the black and blue line represents the actual path $\left(\mathbf{p}=\left[\begin{array}{ll}p_{x} & p_{y}\end{array}\right]^{T}\right)$ of the SFC tip for electromagnetic (EM)- and ultrasound-guided instrument tip stabilization experiments, respectively. 
error in the $y$-axis (Fig. 4.8 , around $2 \mathrm{sec}$ ) for EM-guided SFC tip stabilization of US pre-operative patient data (30 BPM). This could be caused by the interaction between the catheter and the opening and closing MV valve, which acts as a disturbance. However, alternative contributions to the stabilization error could be cross-talk between the tendons and mechanical friction in the steering mechanism of the SFC.

\subsection{Conclusions and future work}

In this study, we have presented and demonstrated a novel approach to stabilize the SFC tip for beating heart MV motions. We describe EM- and US-guided control methods to stabilize the SFC tip for MV motions in a realistic and functional MV model. Further, we developed a catheter steering module capable of robot actuation of a wide range of tendon-driven catheters. Experiments are conducted to evaluate the accuracy of SFC tip stabilization. In order to provide a realistic experimental scenario, the SFC tip stabilization is evaluated in an acrylic heart system with an embedded and functional MV model, which is attached to a 6DOF Stewart platform. The Stewart platform is used to reproduce beating heart motions based on pre-operative patient data, while a pulsatile pump is used to provide a fluid flow, which enables opening and closing of the realistic MV model. The experiments with a reproduced heart rate of $30 \mathrm{BPM}$ showed a mean absolute distance error of $0.57 \mathrm{~mm}$ and $1.39 \mathrm{~mm}$ for EM- and US-guided SFC tip stabilization methods, respectively.

In future work, we aim to replace the acrylic heart system with an anatomically accurate heart model with an embedded MV. Further, we intend to improve the SFC tip tracking accuracy and provide additional robustness by fusing EM- and US-data. In addition to the aforementioned, the SFC tip stabilization accuracy could benefit from control strategies capable of anticipation and prediction of beating heart MV motions to enable SFC tip stabilization for heart rates up to $60 \mathrm{BPM}$. Nonetheless, experiments showed that the proposed method of autonomous catheter tip stabilization is capable for beating heart motions up to 30 BPM. Therefore, with further developments, future and existing applications in MIS such as MV repair, ablation and cardiovascular biopsies could benefit from improved accuracy and instrument stabilization offered by the proposed methods. 
CHAPTER 5

\title{
A Beating Heart Testbed for the Evaluation of Robotic Cardiovascular Interventions
}

\begin{abstract}
The improved natural hemodynamics offered by mitral valve (MV) repair strategies aims to prevent heart failure and to minimize the use of long-term anticoagulant. This combined with the reduced patient trauma offered by minimally invasive surgical (MIS) interventions, requires an increase in capabilities of MIS MV repair. The use of robotic catheters have been described in MIS applications such as navigational tasks, ablation and MV repair. The majority of the robotic catheters are evaluated in testbeds capable of partially mimicking the cardiac environment (e.g., beating heart motion or relevant anatomy), while the validation of robotic catheters in a clinical scenario is associated with significant preparation time and limited availability. Therefore, continuous catheter development could be aided by an accessible and available testbed capable of reproducing beating heart motions, circulation and the relevant anatomy in MIS cardiovascular interventions. In this study, we contribute a beating heart testbed for the evaluation of robotic catheters in MIS cardiovascular interventions. Our work describes a heart model with relevant interior structures and an integrated realistic MV model, which is attached to a Stewart platform in order to reproduce the beating heart motions based on pre-operative patient data. The beating heart model is extended with an artificial aortic valve, a systemic arterial model, a venous reservoir and a pulsatile pump to mimic the systemic circulation. Experimental evaluation showed systemic circulation and beating heart motion reproduction for $70 \mathrm{BPM}$ with a mean absolute distance error of $1.26 \mathrm{~mm}$, while a robotic catheter in the heart model is observed by ultrasound imaging and electromagnetic position tracking. Therefore, the presented testbed is capable of evaluating MIS robotic cardiovascular interventions such as MV repair, navigation tasks and ablation.
\end{abstract}

This chapter has been previously published as

[145] G.J. Vrooijink, H. Irzan, and S. Misra, A beating heart testbed for the evaluation of robotic cardiovascular interventions, in Proceedings of the IEEE International Conference on Biomedical Robotics and Biomechatronics (BioRob), Enschede, the Netherlands, pages 1076-1082, August 2018. 
Chapter 5. A Beating Heart Testbed for the Evaluation of Robotic Cardiovascular Interventions

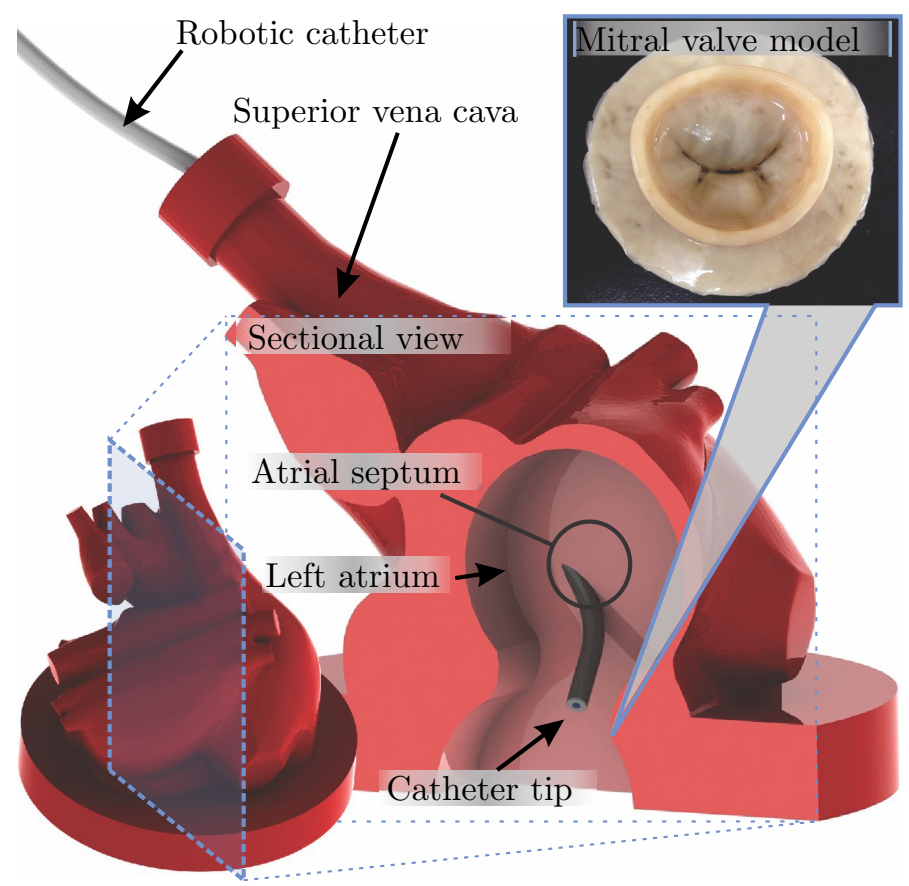

Figure 5.1: A rendering of the beating heart model developed for the evaluation of robotic catheters in minimally invasive mitral valve repair surgery. The interior of the heart is modeled with an anatomically accurate superior vena cava and left and right atria, which is separated by the atrial septum. This allows a robotic catheter to be inserted in the superior vena cava into the right atrium trough the atrial septum to reach a desired treatment location.

\subsection{Introduction}

Mitral insufficiency is one of the commonly observed heart diseases in society, which requires treatment in case of hemodynamic compromise. Mitral valve (MV) repair strategies are often favored over MV replacement strategies [146]. Whereby, repair strategies aim to preserve natural hemodynamics in order to minimize the use of long-term anticoagulant $[13$. MV repair is often performed by open-heart surgery via sternotomy incisions, which is associated with significant patient trauma. Therefore, high-risk patients, often with comorbidities are excluded from surgery 147 . As an alternative to sternotomy incision procedures, MV repair treatment with reduced patient trauma could be provided by minimally invasive surgery (MIS) [15. The favorable long-term outcome provided by MV repair strategies with reduced patient trauma, requires an increase in MIS capability, which can be offered by (robotic) catheter integration 20,148.

The development of robotic catheters for cardiovascular applications have 
been extensively covered in literature. This includes the use of robotic catheters for navigational tasks 130,131,149. Further, work on tele-operated systems, described the deployment of robotic catheters in applications such as endovascular surgery 150,151]. Other studies documented the use of robotic catheters in applications such as ablation and MV repair 72, 78, 80, 110, 152. The majority of aforementioned studies provided results obtained from experimental testbeds, which are used to partially mimic the cardiac environment (e.g., beating heart motion or relevant anatomy), while important aspects of robotic catheter evaluation in cardiovascular surgery such as fluid circulation are neglected. A small group of researchers demonstrated robotic catheter methods in ex- or in-vivo experiments. Validation of robotic catheters in a clinical scenario is associated with significant preparation time and limited availability. Hence, the validation of robotic catheter methods in an accessible and available testbed capable of reproducing the clinical environment could support continuous catheter development.

Various efforts in the modeling of beating heart (sub)system have been described in research. Vismara et al. described a pulsatile mock loop, which is used to simulate the relevant structures and fluid flow for the evaulation of aortic valves $[153$. A left heart simulator was presented by Rabbah et al., which is used for the study of MV mechanics and hemodynamics 154. Mock loops that used various versions of a Windkessel model have been described by a number researchers [155 157]. In these studies, a Windkessel model is used to mimic resistance and compliance of the aorta and arteries of the vascular system. Further, serveral studies presented work capable of repoducing a realistic fluid flow and pressure during the cardiac cycle by using a pulsatile pump 158,159]. Although, the individual components and various beating heart (sub)systems are well described in literature, these studies do not focus on the evaluation of robotically-controlled catheters in cardiovascular surgery. Further, these studies do not combine relevant aspects for robotic catheter evaluation such as beating heart motions, circulation and realistic anatomy in a single testbed.

In this study, we present a beating heart testbed developed for the evaluation of robotic catheters in cardiovascular surgery. Our work contributes a testbed, which comprises of a silicon heart model obtained from a partial cast of a bovine heart (Fig. 5.1). The focus of the testbed design is to provide a realistic environment for minimally invasive MV repair surgery performed by steerable and flexible robotic catheters. Hence, we model the relevant interior heart structures and integrated a realistic and functional MV. In order to reproduce the beating heart motions at the treatment location, we attach the silicon heart model to a six degrees-of-freedom (DOF) Stewart platform. The beating heart motions at the treatment location are reproduced in three dimensional (3D) space by using pre-operative patient data. Further, we extended the beating heart model with an artificial aortic valve (AV) model and a systemic arterial model (SAM) using a Windkessel system in order to mimic the systemic circulation. A pulsatile pump is integrated in order reproduce 
Chapter 5. A Beating Heart Testbed for the Evaluation of Robotic Cardiovascular Interventions

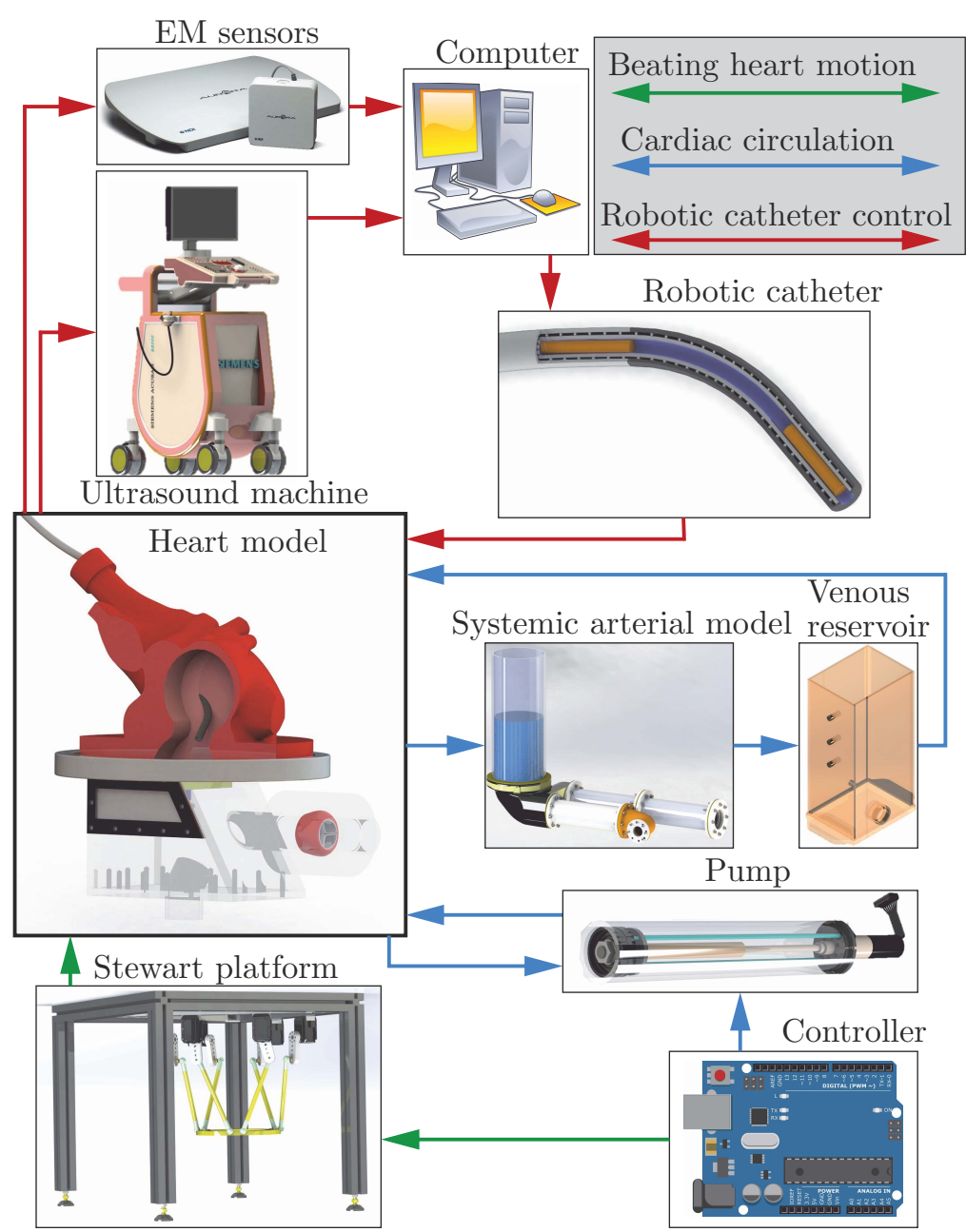

Figure 5.2: An overview of the various components of the beating heart testbed associated with beating-heart MV motion reproduction (green), cardiac circulation (blue) and potential robotic catheter control (red). The heart model comprises of an interior (i.e., superior vena cava, left and right atria and atrial septum), a realistic MV, an acrylic left ventricle and an artificial aortic valve. The heart model is attached to a Stewart platform, which uses a controller to reproduce the beating heart motions based on pre-operative patient data. Systemic circulation is provided by a pulsatile pump, a systemic arterial model and a venous reservoir. A computer controlled robotic catheter could be inserted in the heart model, while feedback is provided by ultrasound images or electromagnetic (EM) position sensors.

the cardiac output by mimicking the change in ventricle volume, which enables opening and closing of the AV and MV models. Hence, the proposed beating heart testbed could provide a realistic environment for MV repair surgery. Note, that other future robotic catheter applications such as navigation, abla- 
tion and cardiac biopsy could potentially be evaluated in the presented beating heart testbed.

\subsection{Methods}

In this section, we provide the methods used to demonstrate the beating heart testbed for the evaluation of robotic catheters in cardiovascular surgery. In section 5.2.1 we present the experimental testbed, while in section 5.2.2 beating heart MV motion modeling is described. Subsequently, in section 5.2.3, we describe the computer-controlled pulsatile pump. In section 5.2.4, we provide details of systemic circulation model. Further, in the derivations presented, we use $k$ to indicate the discrete time variable.

\subsubsection{Experimental testbed}

The beating heart testbed is divided in three major components, which relate to the robotic catheter control in minimally invasive MV repair surgery, the modeling of cardiac circulation and the reproduction of beating heart motions. An overview of the aforementioned components are depicted in Fig. 5.2, while the beating heart testbed is shown in Fig. 5.3

In this study, robotic catheter control in minimally invasive MV repair is considered. Hence, we use silicon heart model obtained from a partial cast of a bovine heart. The interior of the silicon heart is modeled with an anatomical correct superior vena cava and left and right atria, which is separated by a atrial septum. Further, we integrated a functional and realistic MV model with an implemented anterior and posterior middle prolapse (LifeLike BioTissue Inc., London, Canada) in the silicon heart model. This allows for a robotic catheter to be inserted in the superior vena cava into the right atrium trough the atrial septum to reach a desired treatment location such as the MV. By considering a water-filled bellows, the MV including the robotic catheter could be observed by ultrasound imaging as depicted in Fig. 5.2. The water-filled bellows enables the transmission of ultrasound waves from a stationary transducer to the moving heart model during the reproduction of beating heart motions. Note, that a stationary transducer introduces simplifications compared to clinical practice. A fiducial marker is integrated in the testbed as shown in Fig. 5.2, which can be used to evaluate the reproduced beating heart motions of the heart model in ultrasound images. The observed beating heart motion in ultrasound images could be considered as a reference signal for closed-loop control of a robotic catheter. Complementary or as an alternative to ultrasound imaging, electromagnetic (EM) tracking sensors embedded in the catheter could be used for instrument feedback. By minimizing the use of interfering materials such as ferromagnetic and electrically conductive materials in and near the heart model, EM positional tracking of the robotic catheter is not impeded [160]. 
Chapter 5. A Beating Heart Testbed for the Evaluation of Robotic Cardiovascular Interventions

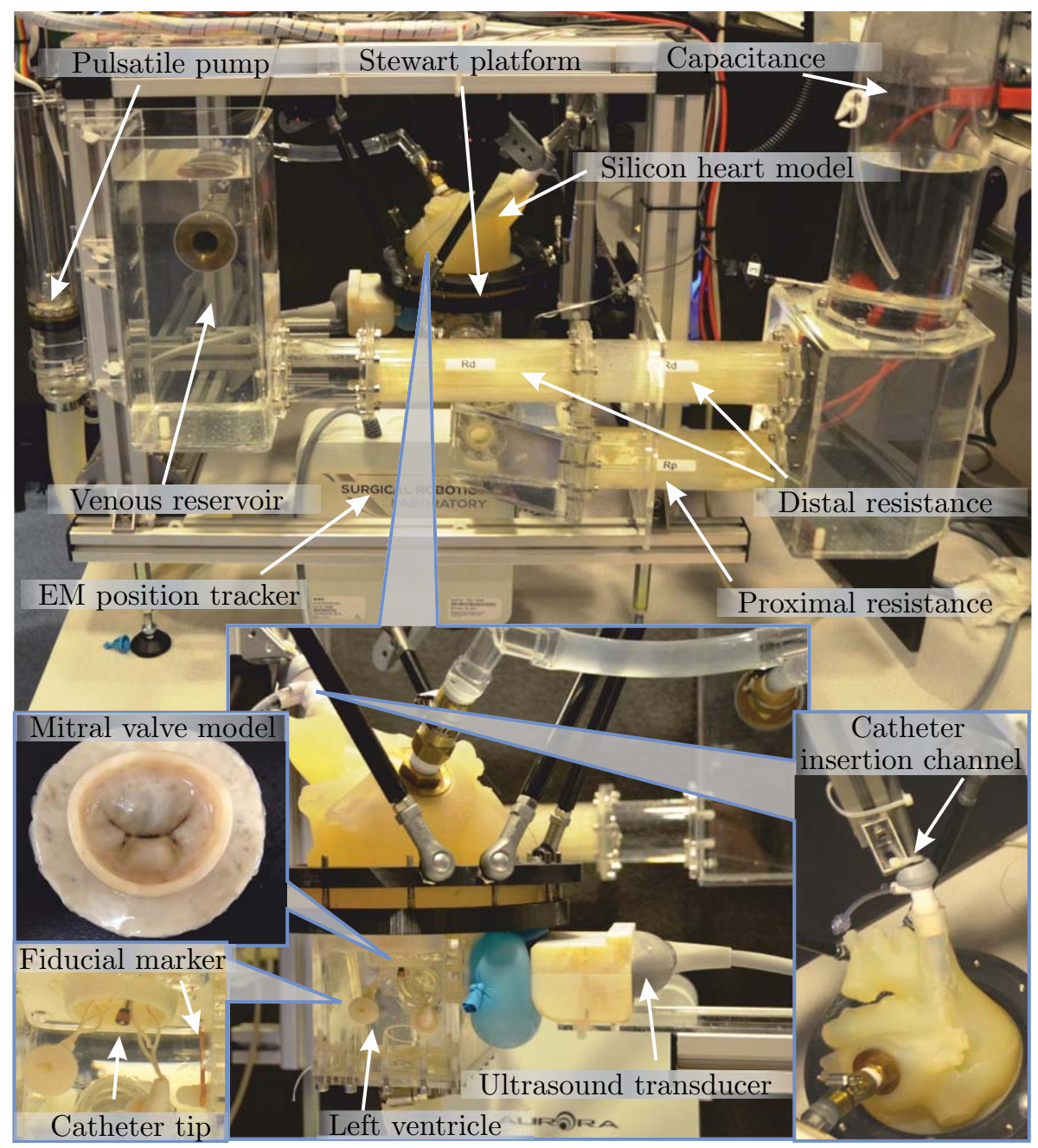

Figure 5.3: An overview of the beating heart testbed for the evaluation of robotic cardiovascular interventions. The beating heart testbed comprises of a silicon heart model with an embedded mitral valve model (bottom-left inset) and an acrylic left ventricle model (bottomcenter inset). In order to mimic systemic fluid flow, the silicon heart model is extended by a pulsatile pump and a systemic arterial model with a proximal resistance, a distal resistance, a capacitance and a venous reservoir. The heart model is attached to a Stewart platform in order to mimic the beating heart motions at the treatment location. Further, a robotic catheter insertion channel (bottom-right inset) provides access to the mitral valve via the superior vena cava, while ultrasound observations and electromagnetic (EM) position tracking are provided.

The functional opening and closing of the integrated MV model is provided by systemic circulation. Hence, we extend the heart model with an acrylic 
left ventricle, an artificial AV and a SAM. The SAM comprises of a proximal resistor, a distal resistor and a capacitance element, which is used to mimic resistance and expansion of the vascular system, respectively. A venous reservoir is included in the systemic circulation to serve as a buffer between the distal resistor and left atrium of the heart model. Note, that by neglecting the pulmonary circulation circuit, we introduce simplifications compared to clinical practice. Further, we attach an automated pump to the left ventricle of the heart model, which is used to reproduce the cardiac output by mimicking the change in ventricle volume. The pump is controlled by an Arduino Due (Arduino, Somerville, USA) and comprises of a piston positioned by a GP22S ball screw spindle drive with a pitch of $2 \mathrm{~mm}$ per rotation, which is attached to a ECMax22 motor (Maxon Motor, Sachseln, Switzerland).

In order to reproduce the beating heart motions at the treatment location (i.e., MV) in 3D space, we attach the heart model to a six DOF Stewart platform 125]. The Stewart platform is actuated by six MX-64AR servo motors (ROBOTIS Co., Ltd., Seoul, South Korea) and controlled by an Arduino Due. The reproduced beating heart motions are obtained from pre-operative patient data by using 3D magnetic resonance images of a MV. Further, we consider the heart-rate variability in the reproduced beating heart motions.

\subsubsection{Beating heart mitral valve motion modeling}

In this section, we describe the model used to reproduce the beating heart MV motions in 3D space, which is obtained by pre-operative patient data using 3D magnetic-resonance (MR) images. The MR volume (seven slices of 256 by 256 pixels) data are obtained with a rate of 30 volumes-per-second by a 1.5 Tesla imaging scanner using a transesophageal echocardiogram (TEE) system (Philips Healthcare, Amsterdam). Manual segmentation is used to obtain the motion path of the MV over the cardiac cycle. More details are provided in previous work, where the beating heart MV motions are reproduction in twodimensional (2D) space 125. The 3D periodic beating heart MV motion is obtained from manual segmentation, which can be described by a two-term Fourier series according to

$$
r_{*}=a_{0_{*}}+a_{1_{*}} c_{\left(\frac{k}{S_{r}} \omega\right)}+b_{1_{*}} s_{\left(\frac{k}{S_{r}} \omega\right)}+a_{2_{*}} c_{\left(2 \frac{k}{S_{r}} \omega\right)}+b_{2_{*}} s_{\left(2 \frac{k}{S_{r}} \omega\right)},
$$

where $*$ denotes the associated $x$-, $y$ - and $z$-axis, $k$ the discrete time variable, $S_{r}$ is the sample rate and $\omega$ denotes the frequency of the periodic motion. However, by considering a constant frequency $(\omega)$ of the periodic beating heart MV motions, the heart rate variability (HRV) is not taken into account. HRV is generated by heart and brain interactions, which is controlled by the autonomic nervous system. HRV is described by the inter-beat interval (IBI), which is the time period between successive heart beats (Fig. 5.4). The HRV frequency is considered to be $<0.4 \mathrm{~Hz}$. Further, the standard deviation (SDNN) of the beatto-beat or NN intervals for patients with SDNN values below 50 milliseconds 
Chapter 5. A Beating Heart Testbed for the Evaluation of Robotic Cardiovascular Interventions

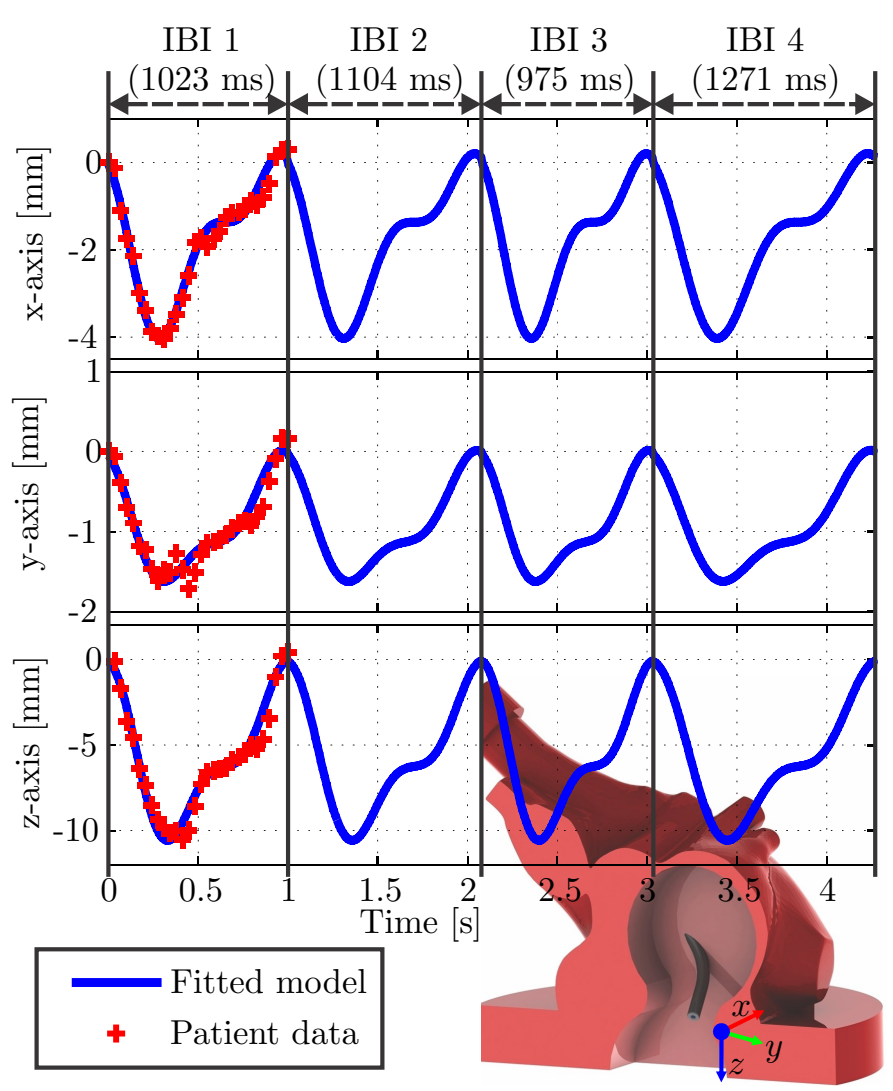

Figure 5.4: The beating heart mitral valve motions obtained from pre-operative patient data (red cross) and the corresponding fitted model (blue line). The fitted model is described by a two-term Fourier series, which is used to reproduce the periodic motion of the beating heart mitral valve in 3D space. Further, heart rate variability modeling is introduced to simulate the beat-to-beat variation, which is indicated by inter-beat intervals (IBI) 1 to 4 .

(ms) are classified as unhealthy, 50 to $100 \mathrm{~ms}$ have compromised health, and above $100 \mathrm{~ms}$ are considered healthy 161. HRV often poses a challenge for the (predictive) control of robotic catheters. Hence, we use a uniform distribution model $\left(\mathcal{U}\left(t_{1}, t_{2}\right)\right)$ to describe the HRV according to

$$
\omega=\omega_{h_{r}}+2 \pi \mathcal{U}\left(t_{1}, t_{2}\right)
$$

where the HRV interval is given by $t_{1}=-200 \mathrm{~ms}$ and $t_{2}=200 \mathrm{~ms}$, while the standard deviation is given by $\frac{1}{2 * \sqrt{3}} *\left(t_{2}-t_{1}\right)=115 \mathrm{~ms}$. Further, the frequency $\left(\omega_{h_{r}}\right)$ can be computed according to $\omega_{h_{r}}=\frac{2 \pi}{60} h_{r}$, where $h_{r}$ is the heart rate in beats-per-minute (BPM), which is used in 5.2 to evaluate the variable frequency $(\omega)$. By considering the variable frequency $(\omega)$ and the twoterm Fourier series in (5.1), we can reproduce beating heart motions with HRV. 


\subsubsection{Cardiac pump}

In order to provide a fluid flow in the heart model, we attach an automated pulsatile pump to the left ventricle of the heart model. The pulsatile pump is used to mimic the left ventricle volume as depicted in Fig. 5.5. The cardiac cycle comprises of systole and diastole phases, where the stroke volume $\left(l_{s v}\right)$ can be evaluated by the end-diastolic volume subtracted by the end-systolic volume. In this study we use a stroke volume of $70 \mathrm{~mL}$, which is observed in clinical practice. By considering the stroke volume $\left(l_{s v}\right)$, the total pump displacement $l_{d}$ is given by

$$
l_{d}=\frac{l_{s v}}{\pi r_{p}^{2}}
$$

where $r_{p}$ is the piston radius. Note, that the ventricle volume changes during the systole (i.e., isovolumic contraction, ejection) and diastole (i.e., isovolumic relaxation, rapid filling, diastasis and atrial systole) phases as depicted in Fig. 5.5 162. Hence, by considering the aforementioned phases and the left ventricle volume as depicted in Fig. 5.5. we used a Fourier series of six terms in order to mimic the ventricle volume $(V(k))$ during the cardiac cycle according to

$$
\begin{gathered}
V(k)=a_{0_{p}}+a_{1_{p}} c_{\left(\frac{k}{S_{r}} \omega\right)}+b_{1_{p}} s_{\left(\frac{k}{S_{r}} \omega\right)}+a_{2_{p}} c_{\left(2 \frac{k}{S_{r}} \omega\right)}+b_{2_{p}} s_{\left(2 \frac{k}{S_{r}} \omega\right)}+ \\
a_{3_{p}} c_{\left(3 \frac{k}{S_{r}} \omega\right)}+b_{3_{p}} s_{\left(3 \frac{k}{S_{r}} \omega\right)}+a_{4_{p}} c_{\left(4 \frac{k}{S_{r}} \omega\right)}+b_{4_{p}} s_{\left(4 \frac{k}{S_{r}} \omega\right)}+ \\
a_{5_{p}} c_{\left(5 \frac{k}{S_{r}} \omega\right)}+b_{5_{p}} s_{\left(5 \frac{k}{S_{r}} \omega\right)}+a_{6{ }_{p}} c_{\left(6 \frac{k}{S_{r}} \omega\right)}+b_{6 p} s_{\left(6 \frac{k}{S_{r}} \omega\right)},
\end{gathered}
$$

where $s_{(}(*)=\sin (*)$ and $\left.c_{(} *\right)=\cos (*)$, the Fourier coefficients are given by $a_{0_{p}}=35.03, a_{1_{p}}=24.34, b_{1_{p}}=-21.77, a_{2_{p}}=6.40, b_{2_{p}}=4.57, a_{3_{p}}=4.36$, $b_{3_{p}}=-0.91, a_{4_{p}}=-0.03, b_{4_{p}}=1.61, a_{5_{p}}=-0.19, b_{5_{p}}=1.06, a_{6_{p}}=-0.24$ and $b_{6_{p}}=1.15$, while the corresponding goodness of fit is given by the square of the correlation $\left(R^{2}=0.99\right)$. Further, the HRV model described $(5.2)$ can be used in the left ventricle volume model provided in (5.4), which is used in systemic circulation reproduction.

Table 5.1: Table of Fourier coefficients used to model the periodic mitral valve motions in (5.1), which are obtained from pre-operative patient data by using 3D magnetic-resonance imaging. Subscript $*$ indicates the associated $x-, y$ and $z$-axis, while the square of the correlation is denoted $\left(R^{2}\right)$.

\begin{tabular}{llllccc}
\hline axis & $a_{0_{*}}$ & $a_{1_{*}}$ & \multicolumn{1}{c}{$b_{1_{*}}$} & $a_{2_{*}}$ & $b_{2_{*}}$ & $R^{2}$ \\
\hline$x$ & -1.735 & 0.888 & -1.413 & 0.804 & -0.006 & 0.98 \\
$y$ & -0.906 & 0.584 & -0.363 & 0.273 & -0.050 & 0.93 \\
$z$ & 5.626 & -3.648 & 2.019 & -1.834 & -0.504 & 0.97 \\
\hline
\end{tabular}


Chapter 5. A Beating Heart Testbed for the Evaluation of Robotic Cardiovascular Interventions

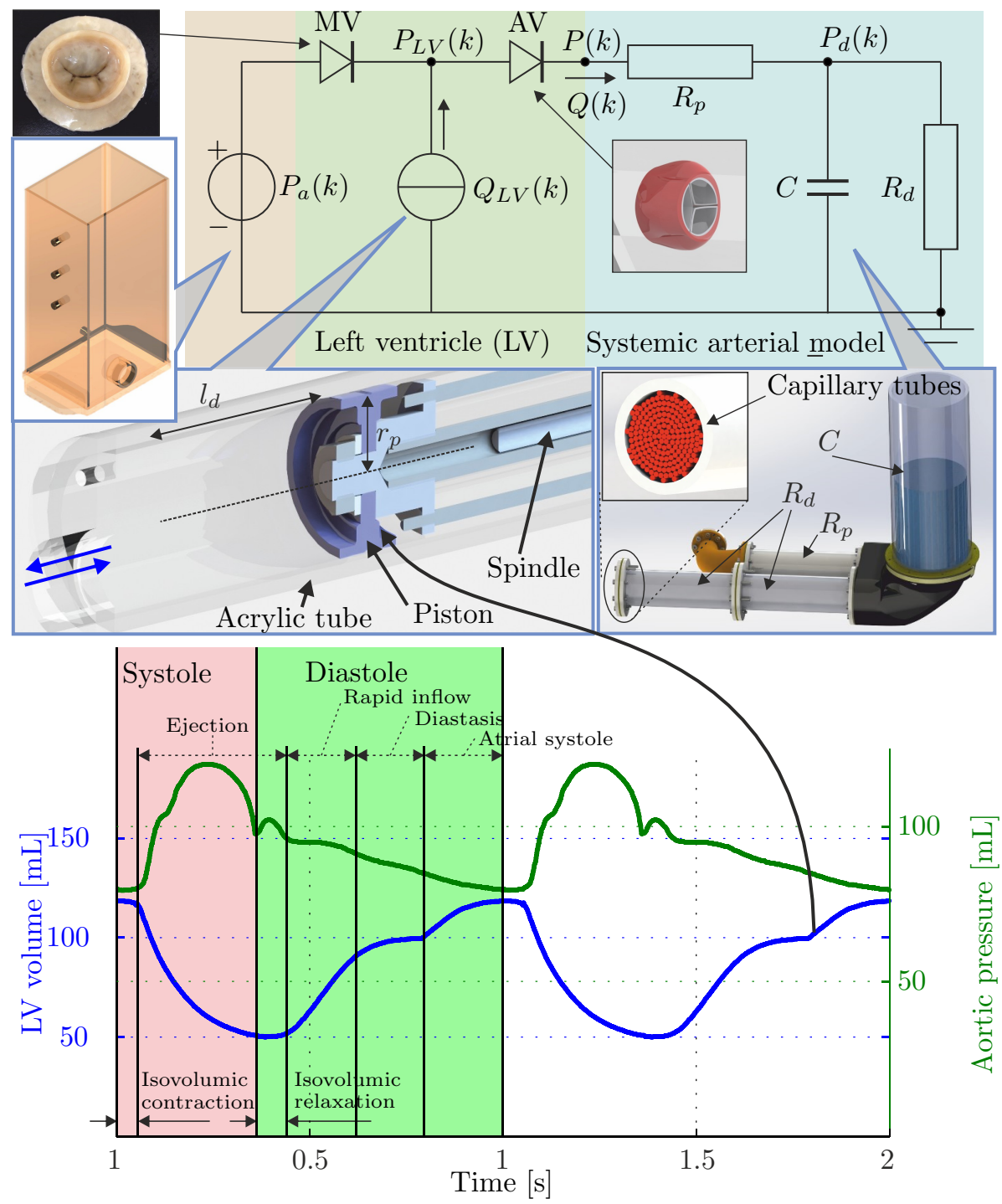

Figure 5.5: An overview of systemic circulation modeling with integrated mitral valve (MV) and aortic valve $(\mathrm{AV})$. A three-element Windkessel model with capacitance $(C)$, proximal resistance $\left(R_{p}\right)$ and distal resistance $\left(R_{d}\right)$ is used to mimic the left ventricle (LV) pressure $\left(P_{L V}(k)\right)$, the aortic pressure $(P(k))$ and distal systemic pressure $\left(P_{d}(k)\right)$, while a fixed filling pressure $\left(P_{a}(k)\right)$ is provided by a venous reservoir (inset left, second from the top). A pulsatile pump with piston radius $\left(r_{p}\right)$ and displacement $\left(l_{d}\right)$ is attached to the LV of the heart model. The pump provides a LV fluid flow $\left(Q_{L V}(k)\right)$ and aortic fluid flow $(Q(k))$, which is described by the time derivative of the LV volume. The bottom inset provides the $\mathrm{LV}$ volume and aortic pressure as described in literature 162 . 


\subsubsection{Systemic circulation modeling}

In order to provide realistic opening and closing of the MV and AV, we mimic the impedance of the systemic arterial system by using a systemic arterial model (SAM). A three-element Windkessel model can be used to describe the pressure and flow relation at the entrance of the systemic arterial system. The SAM as depicted in Fig. 5.5 comprises of a capacitance $(C)$ and proximal and distal resistors, $\left(R_{p}\right)$ and $\left(R_{p}\right)$, respectively. By considering a distal location in the SAM, the relation between the aortic fluid flow $(Q(k))$ and the aortic pressure $(P(k))$ and pressure $\left(P_{d}(k)\right)$ at a distal location is given by

$$
P(k)-P_{d}(k)=R_{p} Q(k) .
$$

Further, the left ventricle volume $(V(k))$ described in $(5.4)$ can be used to find an expression for the fluid flow $(Q(k))$ through the AV entering the SAM according to

$$
Q(k)=\left\{\begin{array}{ll}
-\Delta V(k) & \text { for } k \in\left[t_{n}, t_{n}+t_{s}\right] \\
0 & \text { for } k \in\left[t_{n}+t_{s}, t_{n}+t_{s}+t_{d}\right]
\end{array},\right.
$$

where $t_{n}$ indicates the start time of the cardiac cycle and $t_{s}$ and $t_{d}$ the systole and diastole times, respectively. The relation between fluid flow $(Q(k))$ described in (5.6) and the distal pressure $\left(P_{d}(k)\right)$ is described by a two-element Windkessel model as follows:

$$
Q(k)=C \Delta P_{d}(k)+\frac{1}{R_{d}} P_{d}(k),
$$

where $\Delta P_{d}(k)$ is the discrete time derivative of the distal pressure $\left(P_{d}(k)\right)$. The two-element Windkessel model can be extended to a three-element Windkessel model by rearranging the distal pressure $\left(P_{d}(k)\right)$ in $(5.5)$, which can be substituted into (5.7) according to

$$
C R_{p} \Delta Q(k)+\frac{R_{d}+R_{p}}{R_{d}} Q(k)=C \Delta P(k)+\frac{1}{R d} P(k),
$$

where $\Delta Q(k)$ is the discrete time derivative of the fluid flow $(Q(k))$ described in (5.6), thus know. By considering the differential equation in (5.8), the modeled fluid flow in 5.6 and the aortic pressure described by literature as shown in Fig. (5.5), we can estimate for the proximal resistance $\left(R_{p}=\right.$ $0.075 \mathrm{mmHg} \cdot \mathrm{s} / \mathrm{mL})$, distal resistance $\left(R_{p}=1.25 \mathrm{mmHg} \cdot \mathrm{s} / \mathrm{mL}\right)$ and capacitance $(C=1.9 \mathrm{~mL} / \mathrm{mmHg})$.

The proximal and distal resistances $\left(R_{*}\right)$ can be obtained by $N_{*}$ parallel capillary tubes with length $\left(l_{*}\right)$ and radius $\left(r_{*}\right)$ using the Hagen-Poiseuille equation according to

$$
R_{*}=\frac{P_{*}(k)}{Q_{*}(k)}=\frac{8 \mu l_{*}}{\pi N_{*} r_{*}^{4}} \Rightarrow N_{*}=\frac{8 \mu l_{*}}{\pi R_{*} r_{*}^{4}},
$$


where $*=p$ and $*=d$ are associated with the proximal and distal resistances, respectively. Further, $P_{*}(k)$ describes the pressure loss, $Q_{*}(k)$ is the fluid flow and $\mu$ represents the dynamic viscosity of blood $(0.029 \mathrm{~g} / \mathrm{cm} / \mathrm{s})$. By considering the density of blood $\left(\rho=1060.0 \mathrm{~kg} / \mathrm{m}^{3}\right)$ and the Reynolds number for laminar flow in a capillary tube according to

$$
R e=\frac{2 \rho}{\pi \mu N_{*} r_{*}} Q(k)<2300,
$$

we evaluate the capillary tube lengths $\left(l_{p}=150\right.$ and $\left.l_{d}=300 \mathrm{~mm}\right)$, the radii $\left(r_{p}=0.0775\right.$ and $\left.r_{d}=0.04 \mathrm{~mm}\right)$ and the total parallel tubes $\left(N_{p}=307\right.$ and $\left.N_{d}=519\right)$. Hence, we complete the systemic circulation modeling.

\subsection{Experiments}

In this section, the experimental plan used to evaluate the beating heart testbed is described and the corresponding results are presented.

\subsubsection{Experimental plan}

The experimental plan comprises of three parts. First we evaluate the reproduction of beating heart MV motions including HRV in 3D space based on pre-operative patient data described in section 5.2.2. The reproduced motion is evaluated by using a five DOF EM sensor (Northern Digital Inc., Ontario, Canada), which is attached to the moving Stewart platform. Subsequently, we evaluate the proposed systemic circulation model described in section 5.2 .4 by using MPX5050GP pressure sensors (NXP Semiconductors, Eindhoven, the Netherlands). Finally, we demonstrate ultrasound and EM observation of a stationary robotic catheter inserted in the MV of the beating heart model. The stationary robotic catheter is observed by a Siemens Acuson S2000 ${ }^{\mathrm{TM}}$ ultrasound system with a Siemens 18L6 transducer (Siemens AG, Erlangen, Germany) and by two embedded EM sensors (Northern Digital Inc., Ontario, Canada).

\subsubsection{Results}

The experimental results of the beating heart motion reproduction are provided in Table 5.2. while a representative experiment is depicted in the left plot of Fig. 5.6. Further, the experimental results of system circulation reproduction using a pulsatile pump, a SAM and a venous reservoir are depicted in the center inset (Fig. 5.6), while the observations of a stationary robotic catheter in ultrasound and EM position tracking are provided in the bottom-left and bottom-right insets (Fig. 5.6, respectively.

Experiments in reproducing the beating heart motion show a mean absolute distance error $(\epsilon)$ of approximately $0.8 \mathrm{~mm}$ for heart rates of 20 to $50 \mathrm{BPM}$, 

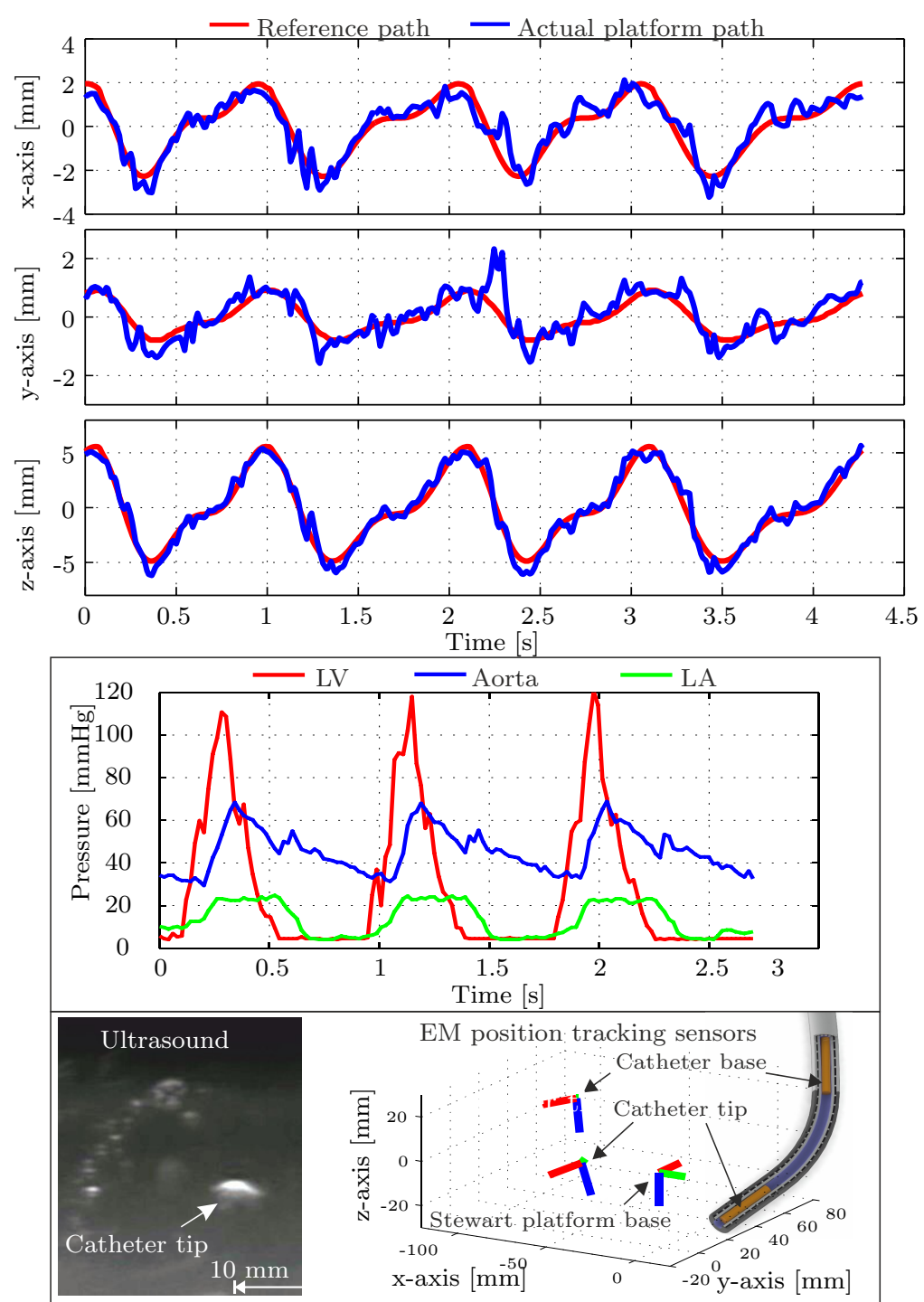

EM position: tracking sensors

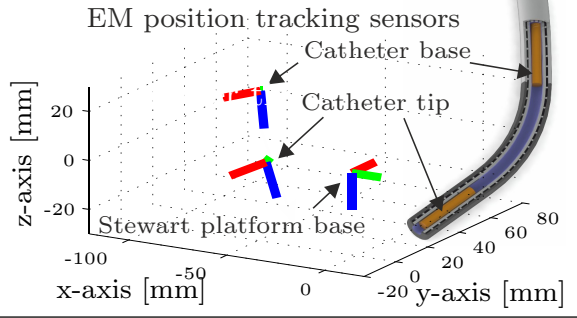

Figure 5.6: Representative experimental results of beating heart motion reproduction in three dimensions (top) and systemic circulation (center) for a heart rate of 60 BPM. The motion reference path is depicted red, while the actual platform path is shown in blue, which is measured by an electromagnetic (EM) sensor attached to the moving Stewart platform. The systemic circulation is provided by a pulsatile pump, while the pressure in the left ventricle (LV) (red), aorta (blue) and left atrium (LA) (blue) are measured. Further, the bottom-left inset demonstrates ultrasound observations and the bottom-right inset shows an EM position measurement of a robotic catheter inserted in the beating heart model. 
Chapter 5. A Beating Heart Testbed for the Evaluation of Robotic Cardiovascular Interventions

Table 5.2: The results of the beating heart testbed motion reproduction experiments for heart rates of 20 to 70 BPM. The mean absolute distance error $(\epsilon)$ and the mean absolute position errors $\left(\epsilon_{x}, \epsilon_{y}\right.$ and $\left.\epsilon_{z}\right)$ are provided with the standard deviation of the absolute error.

\begin{tabular}{ccccc}
\hline $\mathrm{BPM}$ & $\epsilon_{x}[\mathrm{~mm}]$ & $\epsilon_{y}[\mathrm{~mm}]$ & $\epsilon_{z}[\mathrm{~mm}]$ & $\epsilon[\mathrm{mm}]$ \\
\hline 20 & 0.17 & 0.31 & 0.63 & $0.79 \pm 0.53$ \\
30 & 0.26 & 0.38 & 0.57 & $0.80 \pm 0.52$ \\
40 & 0.24 & 0.33 & 0.47 & $0.70 \pm 0.40$ \\
50 & 0.24 & 0.36 & 0.60 & $0.81 \pm 0.57$ \\
60 & 0.33 & 0.49 & 0.78 & $1.07 \pm 0.70$ \\
70 & 0.34 & 0.52 & 0.98 & $1.26 \pm 0.95$ \\
\hline
\end{tabular}

while a deterioration for heart rates of 60 and $70 \mathrm{BPM}$ of 1.07 and $1.26 \mathrm{~mm}$ are observed, respectively. Note, that changing heart rates could be considered during catheter evaluation. The deterioration for higher heart rates (i.e., 60 and $70 \mathrm{BPM}$ ) could potentially be attributed to the tubes between the fixed elements of the SAM and the beating heart model attached to the moving Stewart platform.

Further, systemic circulation experiments showed that the change in LV volume provided by the pulsatile pump results in a maximum LV pressure of approximately $120 \mathrm{mmHg}$, while the observed maximum aortic pressure and the corresponding decay is approximately 70 and $35 \mathrm{mmHg}$, respectively. Note, that the reduced aortic pressure in experiments compared to clinical practice (bottom inset, Fig. 5.5 did not limit AV and MV opening and closing, which is considered in catheter evaluation. The difference between LV and aortic pressure indicates a resistance introduced by the artificial AV, which is similar to AV stenosis. The pressure in the left atrium (LA) during the systole increases to approximately $25 \mathrm{mmHg}$. This could be attributed to the MV with implemented prolapse, which causes MV regurgitation.

Further, observations of a stationary robotic catheter inserted in the beating heart model are provided in Fig. 5.6. Observations showed that the catheter is visible in ultrasound images and could be tracked by two embedded EM position sensors as provided in the bottom inset (Fig. 5.6.

\subsection{Conclusions and future work}

In this study, we described an experimental testbed for the evaluation of robotic cardiovascular interventions. We presented a beating heart model with a realistic interior, a realistic MV and an artificial AV. The heart model is attached to a Stewart platform in order to reproduce the beating heart motions based on pre-operative patient data. Further, systemic circulation is obtained by a pulsatile pump, a SAM and a venous reservoir. This provides a realistic 
cardiac environment for MIS MV repair surgery. In experiments, we demonstrated beating heart motion reproduction with an error of $1.26 \mathrm{~mm}$ for heart rates of $70 \mathrm{BPM}$, while the observed maximum LV and aortic pressures are approximately 120 and $70 \mathrm{mmHg}$, respectively. Further, we provided observations of a stationary catheter in the heart model by using ultrasound images and EM position tracking, which can be used in closed-loop control of robotic instruments.

In future work, we intend to perform robotic catheter studies in the beating heart testbed. Further, we aim to expand the testbed by considering various aortic and mitral valves in order to mimic different cardiac diseases. By considering different realistic valvular models, cardiac decreases such as insufficiency and stenosis could be mimicked, while a robotic catheter could demonstrate effective treatment. Our proposed experimental testbed demonstrates cardiac motion and circulation reproduction with integrated valve models. Therefore, the presented experimental testbed could provide a platform for the evaluation of future robotic catheter applications such as MV repair, navigational tasks and ablation. 
Chapter 5. A Beating Heart Testbed for the Evaluation of Robotic Cardiovascular Interventions 


\section{Part III \\ Outlook}





\section{CHAPTER 6 \\ Conclusions and future work}

The objective of this thesis is to contribute to the development of minimally invasive surgical procedures. The envisioned MIS applications are percutaneous and cardiovascular interventions. The proficient execution of these MIS interventions are often impeded by challenges such as reduced visibility during the procedure and limited dexterity of the instrument at the treatment location. In order to address the aforementioned challenges, methods are developed for the closed-loop control of continuum robots such as needles, delivery sheaths and catheters. In order to analyse the contribution to MIS interventions, the developed continuum robotic methods are evaluated in clinically-relevant testbeds. In this chapter, the conclusions are summarized and potential directions for future work are provided.

\subsection{Conclusions}

In Chapter 2 (Part I) of this thesis, a steering method capable of autonomously and accurately guiding a needle to a target is developed and evaluated. The needle steering method comprises of an ultrasound-based tracker, a needle motion planner, closed-loop controller and a needle insertion device. The ultrasoundbased tracker estimates the needle tip pose by controlling the position of the ultrasound transducer in order to obtain images at the the needle tip. Subsequently, the localization of the needle tip in ultrasound images is performed by a segmentation algorithm, which is used to extract features such as the comettail artifact. The needle tip pose is considered as an input to the needle motion planner, which reacts to perturbations in target and obstacle locations. The updated needle path is provided as a reference to the closed-loop controller. The closed-loop controller is used to steer the needle along the referenced path in order to reach the intended target, while feedback needle tip feedback is provided by the ultrasound-based tracker. Results obtained from experiments showed targeting accuracies of $0.9 \mathrm{~mm}$ and $2.2 \mathrm{~mm}$ for scenarios without obstacles and scenarios with an obstacle moving $7 \mathrm{~mm}$ (typical in breast biopsies), respectively. In literature, it was observed that experienced clinicians in brachytherapy showed an average seed placement error of $6.3 \mathrm{~mm} \mathrm{[108]}$. Furthermore, it was documented that experienced clinicians using rigid needles in prostate biopsies showed average targeting errors between $5.5 \mathrm{~mm}$ and 
$6.5 \mathrm{~mm}$ [109]. Although, the experienced clinicians show less targeting accuracy, the needle steering method described in this thesis is evaluated in softtissue phantoms, which leads to simplifications as opposed to clinical practice. Nevertheless, the proposed needle steering method is technically capable of delivering improved targeting accuracy. Hence, with modification the proposed needle steering method could be applicable to biological tissue, which can be considered a significant contribution to percutaneous MIS interventions.

In Part II of this thesis, methods for cardiovascular interventions using delivery sheaths and catheters are described. In Chapter 3 (Part II), a delivery sheath is steered by model predictive control in order to autonomously and accurately compensate the beating heart motions. An online segmentation algorithm is used to localize the delivery sheath tip position in ultrasound images by filtering, edge detection and a random sample consensus strategy. The localized delivery sheath tip is used for feedback in predictive control. The predictive control strategy with kinematic models is used to compensate aortic heart valve motions based on pre-operative patient data. Furthermore, the predictive control strategy is used to compensate mechanical hysteresis in the system and to restrict instrument motion to an allowable region. By restricting the instrument motion, sensitive tissue is avoided that could be present in surgery. In experiments, evidence is provided that the delivery sheath is capable of tracking aortic heart valve motions with an accuracy of $1.7 \mathrm{~mm}$. However, the system is evaluated in a water container which leads to considerable simplifications compared to clinical practice. Nonetheless, accurate tracking of beating heart motions based on pre-operative patient data is demonstrated. Note, that considerable attention of the surgeon is required in order to accurately track complex repetitive beating heart motions up to 60 beats per minute, while respecting sensitive tissue [19]. Hence, with modifications relevant to clinical practice (e.g., improved robustness), the proposed strategy could provide a significant contribution to minimally invasive cardiovascular surgery.

In Chapter 4 (Part II), the stabilization method described in Chapter 3 (Part II) is extended to the stabilization of a steerable and flexible catheter tip for beating heart mitral valve motions. In addition to ultrasound tracking of the catheter, electromagnetic sensors are embedded at base of the steerable segment and instrument tip in order to provide feedback of the catheter pose for closed-loop control. A novel catheter steering module concept is developed, which is capable of robot actuation of a wide range of tendon driven catheters. The catheter tip is stabilized for beating heart mitral valve motions in a realistic and functional mitral valve model. In order to provide a relevant experimental scenario, the realistic and functional mitral valve model is embedded in a acrylic heart system, which is attached to a 6 DOF Stewart platform. The beating heart mitral valve motions are reproduced by the Stewart platform and based on different pre-operative patient data sources, while a pulsatile pump provided fluid flow to enable opening and closing of the realistic mitral valve model. In 
experiments, catheter tip stabilization is demonstrated up to a reproduced heart rate of 30 beats per minute with an error of $0.6 \mathrm{~mm}$ and $1.4 \mathrm{~mm}$ for closed-loop control using electromagnetic and ultrasound instrument feedback, respectively. Although, an acrylic heart system with fluid flow and embedded realistic and function mitral valve model is used, the anatomy at the testbed treatment location does not describe clinical practice. The absence of realistic anatomy leads to simplifications in the evaluation of continuum robotic steering methods as opposed to clinical practice. Nevertheless, the results obtained by the evaluation of the closed-loop controlled catheter method suggests a system capable of improving minimally invasive surgical interventions. The closedloop controlled catheter offers improved tracking accuracy and uses multiple methods for instrument feedback, which is evaluated in a clinically-relevant testbed.

In Chapter 5 (Part II), an experimental testbed is developed for the evaluation of continuum robotic cardiovascular interventions. The beating heart model comprises of a realistic interior, a realistic mitral valve and an artificial aortic valve. Similar to Chapter 4 (Part II), the heart model is attached to a Stewart platform, which is used to reproduce the beating heart motions based on pre-operative patients data. Furthermore, a systemic circulation circuit is integrated by using a pulsatile pump, a systemic arterial model and a venous reservoir, which are used to reproduce systemic flow in order to enable opening and closing of the heart valves in the system. The beating heart motion reproduction combined with systemic circulations provides a realistic cardiac environment for the evaluation of continuum robots in minimally invasive cardiovascular surgery. In experiments, the reproduction of the beating heart motion is demonstrated with an error of $1.3 \mathrm{~mm}$ for heart rates of 70 beats per minute, while clinically-relevant maximum left ventricular and aortic pressures of approximately 120 and $70 \mathrm{mmHg}$ are observed, respectively. Subsequently, a stationary catheter is introduced in the beating heart model at the treatment location, which is observed by ultrasound images and electromagnetic position tracking. Thus, providing a functional and realistic testbed for the evaluation of closed-loop controlled continuum robots aimed to improve minimally invasive surgery.

\subsection{Future work}

The directions for future work are divided into four topics. First, suggestions for future work related to feedback of continuum robots and clinical environment are described. Subsequently, potential research topics for closed-loop control strategies are presented. Further, possible directions for future work associated with the design, modelling and actuation of continuum robots are provided. Finally, potential research opportunities for the reproduction of clinically-relevant scenarios are given. 
In this work, feedback of continuum robots is provided by ultrasound images and by electromagnetic tracking sensors embedded in the instrument. However, the robustness and accuracy could be improved. The visibility of continuum robots in ultrasound images could be enhanced by using special materials and coatings 163. The enhanced instrument visibility helps segmentation algorithms to extract well known ultrasound imaging artifacts such as reverberation, acoustic shadowing and specular side lobes that are often associated with medical devices. Furthermore, robustness could be provided by segmentation methods that use more than one feature (e.g., shape, structure, size and contrast) for the detection and localization of continuum robots in medical images. Hence, a combination of segmentation algorithms for the extraction of continuum robot artifacts and features in medical images could significantly improve the robustness and the localization accuracy. In addition to instrument feedback provided by ultrasound images and electromagnetic tracking, continuum robot shape measurements for closed-loop control could be provided by integrated fiber bragg grating sensors 36 . Feedback could also be provided by force measurement sensors at relevant locations in the continuum robot, which can be used for interactive control [78. Note, that data fusion methods can be used to combine instrument feedback obtained by multiple sensors (e.g., ultrasound images, electromagnetic tracking, shape sensing and force measurements). By combining instrument measurements from multiple sensors, the robustness and accuracy could be improved. In this work, a reference to the treatment location is provided by a fiducial marker embedded in the heart model. Although, the use of fiducial markers embedded in tissue has been described in clinical practice, the risk of migration should be considered 164, 165. As an alternative, segmentation of medical images could be used to evaluate relevant aspects of the treatment location. Furthermore, a fixed catheter at the treatment location could be used as a reference in medical images. As an example, a reference catheter inserted and clamped in the coronary sinus could potentially provide beating heart mitral valve motion feedback for the stabilization of continuum robots.

Closed-loop control strategies are used to steer continuum robots towards the treatment locations and to stabilize the continuum robot tip for tissue motions. Predictive control is used to stabilize the continuum robot tip for repetitive tissue motions (i.e., respiration and beating heart motions). However, the predictive control strategy could potentially be improved by including data from electrocardiography. This could potentially improve the accuracy of instrument tip stabilization for tissue motions during a procedure performed by a clinician. In order to facilitate a clinician in the loop, shared control by using a tele-operated device should be considered 166. Shared control enables the clinician to perform a procedure, while aid is provided by a control mechanism. As an example, a needle could be inserted or retracted by the clinician, while steering of the instrument is performed by a closed-loop control strategy updated by path planning. Furthermore, interaction between clinician controlling 
a continuum robot and the anatomy should be considered in closed-loop control strategies. Force feedback can be used for interaction, which could potentially provide intuitive control of the continuum robot during the intervention 167].

Various continuum robot designs, models and actuation mechanism are provided in this thesis. For continuum robots such as flexible needles, the torsional stiffness is important for accurate steering 168. In future work, designs should consider torsional stiffness in order to avoid inaccuracies in needle rotations, which is used by steering methods such as duty cycled spinning. Further, manipulation at the base of continuum robots such as steerable and flexible catheters should directly translate to the corresponding instrument tip deflection. However, the catheter tip deflection is often influenced by the instrument shaft configuration. Hence, catheter tip positioning inaccuracies are introduced if the shaft configuration is not considered by modelling or steering algorithms. Future steerable and flexible catheter designs could use mechanisms such as bowden cables in order to decouple the effects of shaft configuration from the instrument tip actuation. Furthermore, in the development of actuation mechanisms for continuum robots, the backdrivability should be considered. The force exerted by actuators (e.g., linear motors) with drive mechanisms for the manipulation of continuum robots could potentially be used in force feedback. As an example, optimal backdrivability is obtained for needles inserted by linear motors. Force feedback can be used for interactive control between clinician and the clinical environment.

In this thesis, the reproduction of clinically-relevant scenarios for percutaneous and cardiovascular applications are provided by soft-tissue phantoms and experimental testbeds with anatomy, respectively. Note, that the proposed instrument steering method described in Chapter 2 (Part I) is evaluated using soft-tissue phantoms, which leads to simplifications as opposed to studies in biological tissue. The interaction between instrument and biological tissue is highly dependent on viscoelastic properties. In many applications, soft tissue elasticity may vary with the anatomical site and often exhibits anisotropic behaviour 169]. The effects of varying tissue elasticity and anisotropic behaviour is not considered in current steering methods. Hence, the use of ex-vivo tissue should be considered. Further, an experimental testbed with relevant anatomy provides an accessible and available method for the evaluation of continuum robots in cardiovascular applications. However, the experimental testbed with relevant anatomy should be expanded to consider different cardiovascular diseases such as degenerative aortic and mitral valve stenosis and regurgitation. By using realistic valvular models, cardiac decreases such as stenosis and regurgitation could be reproduced, while a continuum robot potentially demonstrates effective treatment. Note, that an experimental testbed with relevant anatomy is a simplification of the clinical treatment location. Hence, the presented closed-loop controlled continuum robotic methods should be evaluated in ex-vivo beating heart experiments.

Finally, by considering the results from experimental testbed evaluation as 
described in this thesis, the closed-loop control methods for continuum robots could provide a significant contribution to percutaneous and cardiovascular interventions. The results show accurate positioning and stabilization of continuum robots such as needles and catheters. The work described in this thesis could benefit from the aforementioned suggestions. Hence, with modifications, the proposed methods could be successfully deployed in clinical practice. 


\section{Bibliography}

[1] D. Himbert, F. Descoutures, N. Al-Attar, B. Iung, G. Ducrocq, D. Détaint, E. Brochet, D. Messika-Zeitoun, F. Francis, H. Ibrahim, P. Nataf, and A. Vahanian, "Results of transfemoral or transapical aortic valve implantation following a uniform assessment in high-risk patients with aortic stenosis," Journal of the American College of Cardiology, vol. 54, no. 4, pp. 303-311, 2009.

[2] T. Walther, T. Dewey, M. A. Borger, J. Kempfert, A. Linke, R. Becht, V. Falk, G. Schuler, F. W. Mohr, and M. Mack, "Transapical aortic valve implantation: Step by step," The Annals of Thoracic Surgery, vol. 87, no. 1, pp. 276-283, 2009.

[3] D. R. Wong, J. Ye, A. Cheung, J. G. Webb, R. G. Carere, and S. V. Lichtenstein, "Technical considerations to avoid pitfalls during transapical aortic valve implantation," The Journal of Thoracic and Cardiovascular Surgery, vol. 140, no. 1, pp. 196-202, 2010.

[4] L. H. Cohn, D. H. Adams, G. S. Couper, D. P. Bichell, D. M. Rosborough, S. P. Sears, and S. F. Aranki, "Minimally invasive cardiac valve surgery improves patient satisfaction while reducing costs of cardiac valve replacement and repair," Annals of surgery, vol. 226, no. 4, pp. 421-428, 1997.

[5] S. G. Shulman and D. E. March, "Ultrasound-guided breast interventions: Accuracy of biopsy techniques and applications in patient management," Seminars in Ultrasound, CT, and MRI, vol. 27, no. 4, pp. 298-307, 2006.

[6] Y.-C. Wu, D.-R. Chen, and S.-J. Kuo, "Personal experience of ultrasound-guided 14-gauge core biopsy of breast tumor," European Journal of Surgical Oncology (EJSO), vol. 32, no. 7, pp. 715-718, 2006.

[7] J. H. Youk, E.-K. Kim, M. J. Kim, J. Y. Lee, and K. K. Oh, "Missed breast cancers at us-guided core needle biopsy: How to reduce them," Radiographics, vol. 27, no. 1, pp. 79-94, 2007.

[8] B. Iung, A. Cachier, G. Baron, D. Messika-Zeitoun, F. Delahaye, P. Tornos, C. Gohlke-Bärwolf, E. Boersma, P. Ravaud, and A. Vahanian, "Decision-making in elderly patients with severe aortic stenosis: why are so many denied surgery?," European Heart Journal, vol. 26, no. 24, pp. 2714-2720, 2005. 
[9] J. Ye, A. Cheung, S. V. Lichtenstein, F. Nietlispach, S. Albugami, J.-B. Masson, C. R. Thompson, B. Munt, R. Moss, R. G. Carere, W. R. E. Jamieson, and J. G. Webb, "Transapical transcatheter aortic valve implantation: Follow-up to 3 years," The Journal of Thoracic and Cardiovascular Surgery, vol. 139, no. 5, pp. 1107-1113, 2010.

[10] M. Johansson, S. Nozohoor, P. O. Kimblad, J. Harnek, G. K. Olivecrona, and J. Sjögren, "Transapical versus transfemoral aortic valve implantation: A comparison of survival and safety," The Annals of Thoracic Surgery, vol. 91, no. 1, pp. 57-63, 2011.

[11] Ê. E. Guérios, S. Gloekler, T. Pilgrim, S. Stortecky, L. Büllesfeld, A. A. Khattab, C. Huber, B. Meier, S. Windecker, and P. Wenaweser, "Second valve implantation for the treatment of a malpositioned transcatheter aortic valve," Journal of Invasive Cardiology, vol. 24, no. 9, pp. 457-462, 2012.

[12] R. R. Moss, K. H. Humphries, M. Gao, C. R. Thompson, J. G. Abel, G. Fradet, and B. I. Munt, "Outcome of mitral valve repair or replacement: A comparison by propensity score analysis," Circulation, vol. 108, no. 10 suppl 1, pp. II-90-II-97, 2003.

[13] S. F. Bolling, S. Li, S. M. O'Brien, J. M. Brennan, R. L. Prager, and J. S. Gammie, "Predictors of mitral valve repair: Clinical and surgeon factors," The Annals of Thoracic Surgery, vol. 90, no. 6, pp. 1904-1912, 2010.

[14] J. Seeburger, M. A. Borger, H. Tschernich, S. Leontjev, D. Holzhey, T. Noack, J. Ender, and F. W. Mohr, "Transapical beating heart mitral valve repair," Circulation: Cardiovascular Interventions, vol. 3, no. 6, pp. 611-612, 2010.

[15] J. Y. Woo, E. Rodriguez, P. Atluri, and W. R. Chitwood, Jr, "Minimally invasive, robotic, and off-pump mitral valve surgery," Seminars in Thoracic and Cardiovascular Surgery, vol. 18, no. 2, pp. 139-147, 2006.

[16] O. Alfieri, M. D. Bonis, F. Maisano, and G. L. Canna, "Future directions in degenerative mitral valve repair," Seminars in Thoracic and Cardiovascular Surgery, vol. 19, no. 2, pp. 127-132, 2007.

[17] C. Tamburino, G. P. Ussia, F. Maisano, D. Capodanno, G. La Canna, S. Scandura, A. Colombo, A. Giacomini, I. Michev, S. Mangiafico, V. Cammalleri, M. Barbanti, and O. Alfieri, "Percutaneous mitral valve repair with the mitraclip system: acute results from a real world setting," European Heart Journal, vol. 31, no. 11, pp. 1382-1389, 2010. 
[18] F. Maisano, O. Alfieri, S. Banai, M. Buchbinder, A. Colombo, V. Falk, T. Feldman, O. Franzen, H. Herrmann, S. Kar, K.-H. Kuck, G. Lutter, M. Mack, G. Nickenig, N. Piazza, M. Reisman, C. E. Ruiz, J. Schofer, L. Søndergaard, G. W. Stone, M. Taramasso, M. Thomas, A. Vahanian, J. Webb, S. Windecker, and M. B. Leon, "The future of transcatheter mitral valve interventions: competitive or complementary role of repair vs. replacement?," European Heart Journal, vol. 36, no. 26, pp. 1651$1659,2015$.

[19] V. Falk, "Manual control and tracking a human factor analysis relevant for beating heart surgery," The Annals of Thoracic Surgery, vol. 74, no. 2, pp. 624-628, 2002.

[20] W. R. Chitwood Jr, "Robotic mitral valve surgery: overview, methodology, results, and perspective," Annals of Cardiothoracic Surgery, vol. 5, no. 6 , pp. 544-555, 2016.

[21] N. Abolhassani, R. V. Patel, and M. Moallem, "Needle insertion into soft tissue: A survey," Medical Engineering and Physics, vol. 29, no. 4, pp. 413-431, 2007.

[22] S. P. DiMaio and S. E. Salcudean, "Needle steering and motion planning in soft tissues," IEEE Transactions on Biomedical Engineering, vol. 52, no. 6 , pp. 965-974, 2005.

[23] S. Okazawa, R. Ebrahimi, J. Chuang, S. Salcudean, and R. Rohling, "Hand-held steerable needle device," IEEE/ASME Transactions on Mechatronics, vol. 10, no. 3, pp. 285-296, 2005.

[24] D. S. Minhas, J. A. Engh, M. M. Fenske, and C. N. Riviere, "Modeling of needle steering via duty-cycled spinning," in Proceedings of the IEEE International Conference on Engineering in Medicine and Biology Society (EMBC), pp. 2756-2759, Lyon, France, August 2007.

[25] N. J. Cowan, K. Goldberg, G. Chirikjian, G. Fichtinger, R. Alterovitz, K. B. Reed, V. Kallem, W. Park, S. Misra, and A. M. Okamura, Surgical Robotics, ch. Robotic Needle Steering: Design, Modeling, Planning, and Image Guidance, pp. 557-582. Springer US, 2011.

[26] S.-Y. Ko, L. Frasson, and F. R. y Baena, "Closed-loop planar motion control of a steerable probe with a "programmable bevel" inspired by nature," IEEE Transactions on Robotics, vol. 27, no. 5, pp. 970-983, 2011.

[27] P. E. Dupont, J. Lock, B. Itkowitz, and E. Butler, "Design and control of concentric-tube robots," IEEE Transactions on Robotics, vol. 26, no. 2, pp. 209-225, 2010. 
[28] R. J. Webster, J. S. Kim, N. J. Cowan, G. S. Chirikjian, and A. M. Okamura, "Nonholonomic modeling of needle steering," International Journal of Robotics Research, vol. 25, no. 5-6, pp. 509-525, 2006.

[29] S. Misra, K. B. Reed, B. W. Schafer, K. T. Ramesh, and A. M. Okamura, "Mechanics of flexible needles robotically steered through soft tissue," International Journal of Robotic Research, vol. 29, no. 13, pp. 1640-1660, 2010 .

[30] R. J. Roesthuis, Y. R. J. van Veen, A. Jayha, and S. Misra, "Mechanics of needle-tissue interaction," in Proceedings of the IEEE International Conference on Intelligent Robots and Systems (IROS), pp. 2557-2563, San Francisco, USA, September 2011.

[31] R. J. Webster III and B. A. Jones, "Design and kinematic modeling of constant curvature continuum robots: A review," International Journal of Robotics Research, vol. 29, no. 13, pp. 1661-1683, 2010.

[32] J. Hong, T. Dohi, M. Hashizume, K. Konishi, and N. Hata, "An ultrasound-driven needle-insertion robot for percutaneous cholecystostomy," Physics in Medicine and Biology, vol. 49, no. 3, pp. 441-455, 2004.

[33] R. Webster III, J. Memisevic, and A. Okamura, "Design considerations for robotic needle steering," in Proceedings of the IEEE International Conference on Robotics and Automation (ICRA), pp. 3588-3594, Barcelona, Spain, April 2005.

[34] J. A. Engh, G. Podnar, S. Y. Khoo, and C. N. Riviere, "Flexible needle steering system for percutaneous access to deep zones of the brain," in Proceedings of the IEEE Annual Northeast Bioengineering Conference (NEBEC), pp. 103-104, Easton, Pennsylvania, USA, April 2006.

[35] V. Kallem and N. Cowan, "Image guidance of flexible tip-steerable needles," IEEE Transactions on Robotics, vol. 25, no. 1, pp. 191-196, 2009.

[36] R. J. Roesthuis, N. J. van de Berg, J. J. van den Dobbelsteen, and S. Misra, "Modeling and steering of a novel actuated-tip needle through a soft-tissue simulant using fiber bragg grating sensors," in Proceedings of the IEEE International Conference on Robotics and Automation (ICRA), pp. 2283-2289, Seattle, USA, May 2015.

[37] R. J. Webster, III, J. M. Romano, and N. J. Cowan, "Mechanics of precurved-tube continuum robots," IEEE Transactions on Robotics, vol. 25 , no. 1 , pp. $67-78,2009$.

[38] D. Glozman and M. Shoham, "Image-guided robotic flexible needle steering," IEEE Transactions on Robotics, vol. 23, no. 3, pp. 459-467, 2007. 
[39] P. M. Novotny, J. A. Stoll, N. V. Vasilyev, P. J. del Nido, P. E. Dupont, T. E. Zickler, and R. D. Howe, "GPU based real-time instrument tracking with three-dimensional ultrasound," Medical Image Analysis, vol. 11, no. 5 , pp. 458-464, 2007.

[40] H. R. S. Neshat and R. V. Patel, "Real-time parametric curved needle segmentation in 3d ultrasound images," in Proceedings of the IEEE International Conference on Biomedical Robotics and Biomechatronics (BioRob), pp. 670-675, Scottsdale, USA, October 2008.

[41] Z. Neubach and M. Shoham, "Ultrasound-guided robot for flexible needle steering," IEEE Transactions on Biomedical Engineering, vol. 57, no. 4, pp. $799-805,2010$.

[42] C. Nadeau and A. Krupa, "Intensity-based direct visual servoing of an ultrasound probe," in Proceedings of the IEEE International Conference on Robotics and Automation (ICRA), pp. 5677-5682, Shanghai, China, May 2011.

[43] A. Majewicz, S. P. Marra, M. G. van Vledder, M. Lin, M. A. Choti, D. Y. Song, and A. M. Okamura, "Behavior of tip-steerable needles in ex vivo and in vivo tissue," IEEE Transactions on Biomedical Engineering, vol. 59, no. 10, pp. 2705-2715, 2012.

[44] J. Stoll, H. Ren, and P. Dupont, "Passive markers for tracking surgical instruments in real-time $3 \mathrm{~d}$ ultrasound imaging," IEEE Transactions on Medical Imaging, vol. 31, no. 3, pp. 563-575, 2012.

[45] G. J. Vrooijink, M. Abayazid, and S. Misra, "Real-time three-dimensional flexible needle tracking using two-dimensional ultrasound," in Proceedings of the IEEE International Conference on Robotics and Automation (ICRA), pp. 1680-1685, Karlsruhe, Germany, May 2013.

[46] R. Alterovitz, M. Branicky, and K. Goldberg, "Motion planning under uncertainty for image-guided medical needle steering," International Journal of Robotics Research, vol. 27, no. 11-12, pp. 1361-1374, 2008.

[47] J. Xu, V. Duindam, R. Alterovitz, and K. Goldberg, "Motion planning for steerable needles in 3d environments with obstacles using rapidlyexploring random trees and backchaining," in Proceedings of the IEEE International Conference on Automation Science and Engineering (CASE), pp. 41-46, August 2008.

[48] V. Duindam, J. Xu, R. Alterovitz, S. Sastry, and K. Goldberg, "Threedimensional motion planning algorithms for steerable needles using inverse kinematics," International Journal of Robotics Research, vol. 29, no. 7 , pp. $789-800,2010$. 
[49] W. Park, Y. Wang, and G. Chirikjian, "The path-of-probability algorithm for steering and feedback control of flexible needles," International Journal of Robotics Research, vol. 29, no. 7, pp. 813-830, 2010.

[50] S. Patil and R. Alterovitz, "Interactive motion planning for steerable needles in 3d environments with obstacles," in Proceedings of the IEEE International Conference on Biomedical Robotics and Biomechatronics (BioRob), pp. 893-899, Tokyo, Japan, September 2010.

[51] A. Asadian, M. Kermani, and R. Patel, "Robot-assisted needle steering using a control theoretic approach," Journal of Intelligent and Robotic Systems, vol. 62, no. 3-4, pp. 397-418, 2011.

[52] K. B. Reed, A. Majewicz, V. Kallem, R. Alterovitz, K. Goldberg, N. J. Cowan, and A. M. Okamura, "Robot-assisted needle steering," IEEE Robotics Automation Magazine, vol. 18, no. 4, pp. 35 -46, 2011.

[53] M. Bernardes, B. Adorno, P. Poignet, and G. Borges, "Robot-assisted automatic insertion of steerable needles with closed-loop imaging feedback and intraoperative trajectory replanning," Mechatronics, vol. 23, no. 6, pp. 630-645, 2013.

[54] K. Hauser, R. Alterovitz, N. Chentanez, A. M. Okamura, and K. Goldberg, "Feedback control for steering needles through 3d deformable tissue using helical paths," in Proceedings of Robotics: Science and Systems (RSS), vol. 37, Seattle, USA, June 2009.

[55] M. Abayazid, R. J. Roesthuis, R. Reilink, and S. Misra, "Integrating deflection models and image feedback for real-time flexible needle steering," IEEE Transactions on Robotics, vol. 29, no. 2, pp. 542-553, 2013.

[56] M. Abayazid, M. Kemp, and S. Misra, "3D flexible needle steering in soft-tissue phantoms using fiber bragg grating sensors," in Proceedings of the IEEE International Conference on Robotics and Automation (ICRA), pp. 5823-5829, Karlsruhe, Germany, May 2013.

[57] G. J. Vrooijink, M. Abayazid, S. Patil, R. Alterovitz, and S. Misra, "Needle path planning and steering in a three-dimensional non-static environment using two-dimensional ultrasound images," The International Journal of Robotics Research, vol. 33, no. 10, pp. 1361-1374, 2014.

[58] D. Camarillo, C. Milne, C. Carlson, M. Zinn, and J. Salisbury, "Mechanics modeling of tendon-driven continuum manipulators," IEEE Transactions on Robotics, vol. 24, no. 6, pp. 1262-1273, 2008.

[59] R. J. Roesthuis, S. Janssen, and S. Misra, "On using an array of fiber bragg grating sensors for closed-loop control of flexible minimally invasive 
surgical instruments," in Proceedings of the IEEE International Conference on Intelligent Robots and Systems (IROS), pp. 2545-2551, Tokyo, Japan, November 2013.

[60] Y. Bailly and Y. Amirat, "Modeling and control of a hybrid continuum active catheter for aortic aneurysm treatment," in Proceedings of the IEEE International Conference on Robotics and Automation (ICRA), pp. 924-929, Barcelona, Spain, April 2005.

[61] S. Tully, G. Kantor, M. A. Zenati, and H. Choset, "Shape estimation for image-guided surgery with a highly articulated snake robot," in Proceedings of the IEEE International Conference on Intelligent Robots and Systems (IROS), pp. 1353-1358, San Francisco, USA, September 2011.

[62] N. Simaan, A. Bajo, A. Reiter, L. Wang, P. Allen, and D. Fowler, "Lessons learned using the insertable robotic effector platform (irep) for single port access surgery," Journal of Robotic Surgery, vol. 7, no. 3, pp. 235-240, 2013.

[63] B. Bardou, P. Zanne, F. Nageotte, and M. De Mathelin, "Control of a multiple sections flexible endoscopic system," in Proceedings of the IEEE International Conference on Intelligent Robots and Systems (IROS), pp. 2345-2350, Taipei, Taiwan, October 2010.

[64] A. Degani, H. Choset, A. Wolf, T. Ota, and M. A. Zenati, "Percutaneous intrapericardial interventions using a highly articulated robotic probe," in Proceedings of the IEEE International Conference on Biomedical Robotics and Biomechatronics (BioRob), pp. 7-12, Pisa, Italy, Feb 2006.

[65] J. Ding, K. Xu, R. Goldman, P. Allen, D. Fowler, and N. Simaan, "Design, simulation and evaluation of kinematic alternatives for insertable robotic effectors platforms in single port access surgery," in Proceedings of the IEEE International Conference on Robotics and Automation (ICRA), pp. 1053-1058, Anchorage, USA, May 2010.

[66] J. Ding, R. E. Goldman, K. Xu, P. K. Allen, D. L. Fowler, and N. Simaan, "Design and coordination kinematics of an insertable robotic effectors platform for single-port access surgery.," IEEE/ASME Transactions on Mechatronics, vol. 18, no. 5, pp. 1612-1624, 2013.

[67] A. Reiter, A. Bajo, K. Iliopoulos, N. Simaan, and P. Allen, "Learningbased configuration estimation of a multi-segment continuum robot," in Proceedings of the IEEE International Conference on Biomedical Robotics and Biomechatronics (BioRob), pp. 829-834, Rome, Italy, June 2012.

[68] W. Rone and P. Ben-Tzvi, "Continuum robot dynamics utilizing the principle of virtual power," IEEE Transactions on Robotics, vol. 30, no. 1, pp. 275-287, 2014. 
[69] J. Jayender, M. Azizian, and R. Patel, "Autonomous image-guided robot-assisted active catheter insertion," IEEE Transactions on Robotics, vol. 24 , no. 4 , pp. $858-871,2008$.

[70] Y. Nakamura, K. Kishi, and H. Kawakami, "Heartbeat synchronization for robotic cardiac surgery," in Proceedings of the IEEE International Conference on Robotics and Automation (ICRA), vol. 2, pp. 2014-2019, Seoul, Korea, 2001.

[71] R. Ginhoux, J. Gangloff, M. de Mathelin, L. Soler, M. Sanchez, and J. Marescaux, "Active filtering of physiological motion in robotized surgery using predictive control," IEEE Transactions on Robotics, vol. 21, no. 1, pp. 67-79, 2005.

[72] J. Gangloff, R. Ginhoux, M. de Mathelin, L. Soler, and J. Marescaux, "Model predictive control for compensation of cyclic organ motions in teleoperated laparoscopic surgery," IEEE Transactions on Control Systems Technology, vol. 14, no. 2, pp. 235-246, 2006.

[73] T. Ortmaier, M. Groger, D. Boehm, V. Falk, and G. Hirzinger, "Motion estimation in beating heart surgery," IEEE Transactions on Biomedical Engineering, vol. 52, no. 10, pp. 1729-1740, 2005.

[74] O. Bebek and M. Cavusoglu, "Intelligent control algorithms for roboticassisted beating heart surgery," IEEE Transactions on Robotics, vol. 23, no. 3, pp. 468-480, 2007.

[75] S. Yuen, P. Novotny, and R. Howe, "Quasiperiodic predictive filtering for robot-assisted beating heart surgery," in Proceedings of the IEEE International Conference on Robotics and Automation (ICRA), pp. 38753880, Pasadena, USA, May 2008.

[76] E. Tuna, T. Franke, O. Bebek, A. Shiose, K. Fukamachi, and M. Cavusoglu, "Heart motion prediction based on adaptive estimation algorithms for robotic-assisted beating heart surgery," IEEE Transactions on Robotics, vol. 29, no. 1, pp. 261-276, 2013.

[77] S. G. Yuen, M. C. Yip, N. V. Vasilyev, D. P. Perrin, P. J. del Nido, and R. D. Howe, "Robotic force stabilization for beating heart intracardiac surgery," in Proceedings of the Medical Image Computing and ComputerAssisted Intervention (MICCAI), pp. 26-33, London, UK, September 2009 .

[78] S. B. Kesner and R. D. Howe, "Robotic catheter cardiac ablation combining ultrasound guidance and force control," The International Journal of Robotics Research, vol. 33, no. 4, pp. 631-644, 2014. 
[79] C. Nadeau, H. Ren, A. Krupa, and P. Dupont, "Intensity-based visual servoing for instrument and tissue tracking in 3d ultrasound volumes," IEEE Transactions on Automation Science and Engineering, vol. 12, no. 1, pp. 367-371, 2015.

[80] A. H. Gosline, N. V. Vasilyev, E. J. Butler, C. Folk, A. Cohen, R. Chen, N. Lang, P. J. del Nido, and P. E. Dupont, "Percutaneous intracardiac beating-heart surgery using metal mems tissue approximation tools," The International Journal of Robotics Research, vol. 31, no. 9, pp. 1081-1093, 2012 .

[81] M. Bowthorpe, A. Alvarez Garcia, and M. Tavakoli, "GPC-based teleoperation for delay compensation and disturbance rejection in image-guided beating-heart surgery," in Proceedings of the IEEE International Conference on Robotics and Automation (ICRA), pp. 4875-4880, Hong Kong, China, May 2014.

[82] P. Breedveld, "Multi-steerable cardiology instruments - multi." https://www.bitegroup.nl/category/research-projects/multi/, 2019.

[83] J. Dankelman, "Final report perspectief program instruments for minimally invasive techniques (imit)." https://www.nwo.nl/binaries/content/documents/nwo/algemeen/ documentation/application/ttw/perspectief/final-report-imit/ Final+report+Perspectief-programma+iMIT, 2019.

[84] W. Bogdanich, "At V.A. Hospital, a Rogue Cancer Unit." http://www.nytimes.com/2009/06/21/health/21radiation.html, 2009.

[85] E. E. Deurloo, K. G. Gilhuijs, L. J. S. Kool, and S. H. Muller, "Displacement of breast tissue and needle deviations during stereotactic procedures," Investigative Radiology, vol. 36, no. 6, pp. 347-353, 2001.

[86] J. Op den Buijs, M. Abayazid, C. L. de Korte, and S. Misra, "Target motion predictions for pre-operative planning during needle-based interventions," in Proceedings of the IEEE International Conference on Engineering in Medicine and Biology Society (EMBC), pp. 5380-5385, Boston, Massachusetts, USA, September 2011.

[87] J. Op den Buijs, H. H. G. Hansen, R. G. P. Lopata, C. L. de Korte, and S. Misra, "Predicting target displacements using ultrasound elastography and finite element modeling," IEEE Transactions on Biomedical Engineering, vol. 58, no. 11, pp. 3143-3155, 2011.

[88] M. Abayazid, J. Buijs, op den, C. Korte, de, and S. Misra, "Effect of skin thickness on target motion during needle insertion into soft-tissue phantoms," in Proceedings of the IEEE International Conference on Biomed- 
ical Robotics and Biomechatronics (BioRob), pp. 755-760, Rome, Italy, June 2012.

[89] D. J. Brenner and E. J. Hall, "Computed tomographyan increasing source of radiation exposure," New England Journal of Medicine, vol. 357, no. 22, pp. 2277-2284, 2007.

[90] H. Fred, "Drawbacks and limitations of computed tomography: views from a medical educator," Texas Heart Institute Journal, vol. 31, no. 4, pp. 345-348, 2004.

[91] S. P. DiMaio, E. Samset, G. Fischer, I. Iordachita, G. Fichtinger, F. Jolesz, and C. M. Tempany, "Dynamic mri scan plane control for passive tracking of instruments and devices," in Proceedings of the International Conference on Medical Image Computing and Computer-Assisted Intervention (MICCAI), pp. 50-58, Brisbane, Australia, October 2007.

[92] N. D. Glossop, K. Cleary, L. Kull, and F. Banovac, "Needle tracking using the aurora magnetic position sensor," in Proceedings of the International Society for Computer Assisted Orthopaedic Surgery (CAOS), pp. 90-92, Santa Fe, USA, June 2002.

[93] J. van den Berg, S. Patil, R. Alterovitz, P. Abbeel, and K. Goldberg, "LQG-based planning, sensing, and control of steerable needles," in Workshop on Algorithmic Foundations of Robotics (WAFR), pp. 373389, Singapore, December 2010.

[94] S. Patil, J. van den Berg, and R. Alterovitz, "Motion planning under uncertainty in highly deformable environments," in Proceedings of Robotics: Science and Systems (RSS), Los Angeles, CA, USA, June 2011.

[95] J. E. Aldrich, "Basic physics of ultrasound imaging," Critical Care Medicine, vol. 35, no. 5, pp. S131-S137, 2007.

[96] J. Huang, J. Triedman, N. Vasilyev, Y. Suematsu, R. Cleveland, and P. Dupont, "Imaging artifacts of medical instruments in ultrasoundguided interventions," Journal of Ultrasound in Medicine, vol. 26, no. 10, pp. 1303-1322, 2007.

[97] Y. Bar-Shalom, X. R. Li, and T. Kirubarajan, Estimation with Applications to Tracking and Navigation. New York, USA: John Wiley \& Sons., 2001.

[98] S. M. LaValle, Planning Algorithms. Cambridge University Press, 2006. Available at http://planning.cs.uiuc.edu. 
[99] A. Shkolnik, M. Walter, and R. Tedrake, "Reachability guided sampling for planning under differential constraints," in Proceedings of the IEEE International Conference on Robotics and Automation (ICRA), pp. 28592865, Kobe, Japan, May 2009.

[100] G. Taubin, "Estimation of planar curves, surfaces, and nonplanar space curves defined by implicit equations with applications to edge and range image segmentation," IEEE Transactions on Pattern Analysis and Machine Intelligence, vol. 13, no. 11, pp. 1115-1138, 1991.

[101] Y. R. J. van Veen, A. Jahya, and S. Misra, "Macroscopic and microscopic observations of needle insertion into gels," Proceedings of the Institution of Mechanical Engineers, Part H: Journal of Engineering in Medicine, vol. 226 , no. 6 , pp. 441-449, 2012.

[102] A. Gefen and B. Dilmoney, "Mechanics of the normal woman's breast," Technology and Health Care, vol. 15, no. 4, pp. 259-271, 2007.

[103] J. R. Cook, R. R. Bouchard, and S. Y. Emelianov, "Tissue-mimicking phantoms for photoacoustic and ultrasonic imaging," Biomedical Optics Express, vol. 2, no. 11, pp. 3193-3206, 2011.

[104] Y. Tomozawa, Y. Inaba, H. Yamaura, Y. Sato, M. Kato, T. Kanamoto, and M. Sakane, "Clinical value of ct-guided needle biopsy for retroperitoneal lesions," Korean Journal of Radiology, vol. 12, no. 3, pp. 351-357, 2011.

[105] M. A. C. Onuigbo, M. E. Cuffy-Hallam, N. A. Dunsmore, and E. S. Zinreich, "Mammography reveals a 2-mm intraductal breast carcinoma," Hospital Physician, vol. 37, no. 5, pp. 61-64, 2001.

[106] P.-J. D. Vos, "Effect of system parameters on target motion," bachelor's degree in advanced technology, Faculty of Science and Technology, University of Twente, The Netherlands, Januari 2013.

[107] U. Güth, D. J. Huang, M. Huber, A. Schötzau, D. Wruk, W. Holzgreve, E. Wight, and R. Zanetti-Dällenbach, "Tumor size and detection in breast cancer: Self-examination and clinical breast examination are at their limit," Cancer Detection and Prevention, vol. 32, no. 3, pp. 224-228, 2008.

[108] R. Taschereau, J. Pouliot, J. Roy, and D. Tremblay, "Seed misplacement and stabilizing needles in transperineal permanent prostate implants," Radiotherapy and Oncology, vol. 55, no. 1, pp. 59-63, 2000.

[109] P. Blumenfeld, N. Hata, S. DiMaio, K. Zou, S. Haker, G. Fichtinger, and C. M. Tempany, "Transperineal prostate biopsy under magnetic resonance image guidance: A needle placement accuracy study," Journal of Magnetic Resonance Imaging, vol. 26, no. 3, pp. 688-694, 2007. 
[110] G. J. Vrooijink, A. Denasi, J. G. Grandjean, and S. Misra, "Model predictive control of a robotically actuated delivery sheath for beating heart compensation," The International Journal of Robotics Research, vol. 36, no. 2, pp. 193-209, 2017.

[111] G. J. Vrooijink, T. T. M. Ellenbroek, P. Breedveld, J. G. Grandjean, and S. Misra, "A preliminary study on using a robotically-actuated delivery sheath (rads) for transapical aortic valve implantation," in Proceedings of the IEEE International Conference on Robotics and Automation (ICRA), pp. 4380-4386, Hong Kong, China, June 2014.

[112] A. Alwan et al., Global status report on noncommunicable diseases 2010. World Health Organization, 2011.

[113] A. Cribier, H. Eltchaninoff, C. Tron, F. Bauer, C. Agatiello, L. Sebagh, A. Bash, D. Nusimovici, P. Litzler, J.-P. Bessou, and M. B. Leon, "Early experience with percutaneous transcatheter implantation of heart valve prosthesis for the treatment of end-stage inoperable patients with calcific aortic stenosis," Journal of the American College of Cardiology, vol. 43, no. 4, pp. 698-703, 2004.

[114] R. Richa, A. Bó, and P. Poignet, "Beating heart motion prediction for robust visual tracking," in Proceedings of the IEEE International Conference on Robotics and Automation (ICRA), pp. 4579-4584, Anchorage, USA, May 2010.

[115] R. Roy, L. Wang, and N. Simaan, "Modeling and estimation of friction, extension, and coupling effects in multisegment continuum robots," IEEE/ASME Transactions on Mechatronics, vol. 22, no. 2, pp. 909-920, 2017.

[116] A. Orekhov, C. Abah, and N. Simaan, "Snake-like robots for minimally invasive, single port, and intraluminal surgeries," Encyclopedia of Medical Robotics, vol. 1, pp. 203-243, 2018.

[117] A. B. Koolwal, F. Barbagli, C. Carlson, and D. Liang, "An ultrasoundbased localization algorithm for catheter ablation guidance in the left atrium," The International Journal of Robotics Research, vol. 29, no. 6, pp. 643-665, 2010.

[118] N. Simaan, R. Taylor, and P. Flint, "A dexterous system for laryngeal surgery," in Proceedings of the IEEE International Conference on Robotics and Automation (ICRA), pp. 351-357, New Orleans, USA, April 2004.

[119] D. A. Forsyth and J. Ponce, Computer vision a modern approach. Prentice Hall Professional Technical Reference, 2002. 
[120] T. van den Boom and A. A. Stoorvogel, "Model predictive control." Lecture Notes for the Dutch Institute of Systems and Control (DISC), The Netherlands, January 2012.

[121] M. Kinnaert, "Adaptive generalized predictive controller for mimo systems," International Journal of Control, vol. 50, no. 1, pp. 161-172, 1989.

[122] H. Ferreau, C. Kirches, A. Potschka, H. Bock, and M. Diehl, "qpOASES: A parametric active-set algorithm for quadratic programming," Mathematical Programming Computation, vol. 6, no. 4, pp. 327-363, 2014.

[123] R. Reilink, S. Stramigioli, and S. Misra, "Image-based hysteresis reduction for the control of flexible endoscopic instruments," Mechatronics, vol. 23 , no. 6 , pp. $652-658,2013$.

[124] P. Breedveld, "Steerable laparoscopic cable-ring forceps," in Proceedings of the Design of Medical Devices Conference (DMD), p. 7, Minneapolis, MN, USA, April 2010.

[125] G. J. Vrooijink, M. P. Jansen, M. L. Tolhuisen, J. G. Grandjean, and S. Misra, "Ultrasound-guided stabilization of a robotically-actuated delivery sheath (rads) for beating heart mitral valve motions," in Proceedings of the IEEE International Conference on Biomedical Robotics and Biomechatronics, pp. 73-79, Singapore, June 2016.

[126] P. Breedveld, J. S. Sheltes, E. M. Blom, and J. E. I. Verheij, "A new, easily miniaturized steerable endoscope," IEEE Engineering in Medicine and Biology Magazine, vol. 24, no. 6, pp. 40-47, 2005.

[127] A. Loeve, P. Breedveld, and J. Dankelman, "Scopes too flexible...and too stiff," IEEE Pulse, vol. 1, no. 3, pp. 26-41, 2010.

[128] E. S. Gang, B. L. Nguyen, Y. Shachar, L. Farkas, L. Farkas, B. Marx, D. Johnson, M. C. Fishbein, C. Gaudio, and S. J. Kim, "Dynamically shaped magnetic fieldsclinical perspective," Circulation: Arrhythmia and Electrophysiology, vol. 4, no. 5, pp. 770-777, 2011.

[129] K. J. Boskma, S. Scheggi, and S. Misra, "Closed-loop control of a magnetically-actuated catheter using two-dimensional ultrasound images," in Proceedings of the IEEE International Conference on Biomedical Robotics and Biomechatronics (BioRob), pp. 61-66, Singapore, June 2016.

[130] Y. Ganji, F. Janabi-Sharifi, and A. N. Cheema, "Robot-assisted catheter manipulation for intracardiac navigation," International Journal of Computer Assisted Radiology and Surgery, vol. 4, no. 4, pp. 307-315, 2009. 
[131] J. Jayender, R. V. Patel, and S. Nikumb, "Robot-assisted active catheter insertion: Algorithms and experiments," The International Journal of Robotics Research, vol. 28, no. 9, pp. 1101-1117, 2009.

[132] J. W. Park, J. Choi, H.-N. Pak, S. J. Song, J. C. Lee, Y. Park, S. M. Shin, and K. Sun, "Development of a force-reflecting robotic platform for cardiac catheter navigation," Artificial Organs, vol. 34, no. 11, pp. 1034$1039,2010$.

[133] Y. Fu, A. Gao, H. Liu, and S. Guo, "The master-slave catheterisation system for positioning the steerable catheter," International Journal of Mechatronics and Automation, vol. 1, no. 3-4, pp. 143-152, 2011.

[134] M. A. Tavallaei, Y. Thakur, S. Haider, and M. Drangova, "A magneticresonance-imaging-compatible remote catheter navigation system," IEEE Transactions on Biomedical Engineering, vol. 60, pp. 899-905, April 2013.

[135] B. Conrad and M. Zinn, "Closed loop task space control of an interleaved continuum-rigid manipulator," in Proceedings of the IEEE International Conference on Robotics and Automation (ICRA), pp. 1743-1750, Seattle, USA, May 2015.

[136] P. Loschak, L. Brattain, and R. Howe, "Automated pointing of cardiac imaging catheters," in Proceedings of the IEEE International Conference on Robotics and Automation (ICRA), pp. 5794-5799, Karlsruhe, Germany, May 2013.

[137] N. V. Vasilyev, A. H. Gosline, E. Butler, N. Lang, P. J. Codd, H. Yamauchi, E. N. Feins, C. R. Folk, A. L. Cohen, R. Chen, D. Zurakowski, P. J. del Nido, and P. E. Dupont, "Percutaneous steerable robotic tool delivery platform and metal microelectromechanical systems device for tissue manipulation and approximation: Closure of patent foramen ovale in an animal model.," Circulation. Cardiovascular interventions, vol. 6, no. 4 , pp. $468-475,2013$.

[138] N. V. Vasilyev, A. H. Gosline, A. Veeramani, M. T. Wu, G. P. Schmitz, R. T. Chen, V. Arabagi, P. J. del Nido, and P. E. Dupont, "Tissue removal inside the beating heart using a robotically delivered metal mems tool," The International Journal of Robotics Research, vol. 34, no. 2, pp. 236$247,2015$.

[139] W. Bachta, P. Renaud, E. Laroche, A. Forgione, and J. Gangloff, "Active stabilization for robotized beating heart surgery," IEEE Transactions on Robotics, vol. 27, no. 4, pp. 757-768, 2011.

[140] J. Zhu, Optimization of Matching Layer Design for Medical Ultrasonic Transducer. PhD thesis, Pennsylvania State University, 2008. 
[141] U. A. van der Heide, A. N. Kotte, H. Dehnad, P. Hofman, J. J. Lagenijk, and M. van Vulpen, "Analysis of fiducial marker-based position verification in the external beam radiotherapy of patients with prostate cancer," Radiotherapy and Oncology, vol. 82, no. 1, pp. 38-45, 2007.

[142] E. Fichter, "A stewart platform-based manipulator: General theory and practical construction," The International Journal of Robotics Research, vol. 5, no. 2, pp. 157-182, 1986.

[143] M. L. Tolhuisen, "Three-dimensional spatial movement of the mitral valve." Master's degree in Technical Medicine (Internship Report), Faculty of Science and Technology, University of Twente, the Netherlands, February 2015.

[144] M. P. Jansen, "Three-dimensional translation and rotation of the mitral valve during the cardiac cycle." Master's degree in Technical Medicine (Internship Report), Faculty of Science and Technology, University of Twente, the Netherlands, November 2015.

[145] G. J. Vrooijink, H. Irzan, and S. Misra, "A beating heart testbed for the evaluation of robotic cardiovascular interventions," in Proceedings of the IEEE International Conference on Biomedical Robotics and Biomechatronics (Biorob), pp. 1076-1082, Enschede, the Netherlands, Aug 2018.

[146] A. B. Goldstone, J. E. Cohen, J. L. Howard, B. B. Edwards, A. L. Acker, W. Hiesinger, J. W. M. Jr., P. Atluri, and J. Y. Woo, "A "repair-all" strategy for degenerative mitral valve disease safely minimizes unnecessary replacement," The Annals of Thoracic Surgery, vol. 99, no. 6, pp. 1983-1991, 2014.

[147] O. De Backer, N. Piazza, S. Banai, G. Lutter, F. Maisano, H. C. Herrmann, O. W. Franzen, and L. Søndergaard, "Percutaneous transcatheter mitral valve replacement," Circulation: Cardiovascular Interventions, vol. 7, no. 3, pp. 400-409, 2014.

[148] D. A. Murphy, E. Moss, J. Binongo, J. S. Miller, S. K. Macheers, E. L. Sarin, A. M. Herzog, V. H. Thourani, R. A. Guyton, and M. E. Halkos, "The expanding role of endoscopic robotics in mitral valve surgery: 1,257 consecutive procedures," The Annals of Thoracic Surgery, vol. 100, no. 5, pp. 1675-1682, 2015.

[149] N. Xiao, P. Guo, and S. Guo, "Push force feedback for a kind of robotic catheter navigation system," in Proceedings of the IEEE International Conference on Information and Automation, pp. 32-37, Lijiang, China, August 2015. 
[150] X. Yin, S. Guo, H. Hirata, and H. Ishihara, "Design and experimental evaluation of a teleoperated haptic robot-assisted catheter operating system," Journal of Intelligent Material Systems and Structures, vol. 27, no. 1, pp. 3-16, 2016.

[151] B. Rosa, A. Devreker, H. D. Praetere, C. Gruijthuijsen, S. Portoles-Diez, A. Gijbels, D. Reynaerts, P. Herijgers, J. V. Sloten, and E. V. Poorten, "Intuitive teleoperation of active catheters for endovascular surgery," in Proceedings of the IEEE International Conference on Intelligent Robots and Systems (IROS), pp. 2617-2624, Hamburg, Germany, September 2017.

[152] P. M. Loschak, A. Değirmenci, and R. D. Howe, "Predictive filtering in motion compensation with steerable cardiac catheters," in Proceedings of the IEEE International Conference on Robotics and Automation (ICRA), pp. 4830-4836, Singapore, May 2017.

[153] R. Vismara, G. B. Fiore, A. Mangini, M. Contino, M. Lemma, A. Redaelli, and C. Antona, "A novel approach to the in vitro hydrodynamic study of the aortic valve: Mock loop development and test," American Society for Artificial Internal Organs, vol. 56, no. 4, pp. 279$284,2010$.

[154] J.-P. Rabbah, N. Saikrishnan, and A. P. Yoganathan, "A novel left heart simulator for the multi-modality characterization of native mitral valve geometry and fluid mechanics," Annals of biomedical engineering, vol. 41, no. 2, pp. 305-315, 2013.

[155] N. Westerhof, J.-W. Lankhaar, and B. E. Westerhof, "The arterial windkessel," Medical $\&$ Biological Engineering 8 Computing, vol. 47, no. 2, pp. 131-141, 2009.

[156] E. O. Kung and C. A. Taylor, "Development of a physical windkessel module to re-create in-vivo vascular flow impedance for in-vitro experiments," Cardiovascular engineering and technology, vol. 2, no. 1, pp. 2$14,2011$.

[157] K. Her, J. Y. Kim, K. M. Lim, and S. W. Choi, "Windkessel model of hemodynamic state supported by a pulsatile ventricular assist device in premature ventricle contraction," BioMedical Engineering OnLine, vol. 17, no. 1, pp. 1-13, 2018.

[158] R. A. Chaudhury, V. Atlasman, G. Pathangey, N. Pracht, R. J. Adrian, and D. H. Frakes, "A high performance pulsatile pump for aortic flow experiments in 3-dimensional models," Cardiovascular Engineering and Technology, vol. 7, no. 2, pp. 148-158, 2016. 
[159] R. R. Mechoor, T. Schmidt, and E. Kung, "A real-time programmable pulsatile flow pump for in vitro cardiovascular experimentation," Journal of Biomechanical Engineering, vol. 138, no. 11, pp. 1-5, 2016.

[160] A. M. Franz, T. Haidegger, W. Birkfellner, K. Cleary, T. M. Peters, and L. Maier-Hein, "Electromagnetic tracking in medicine - a review of technology, validation, and applications," IEEE Transactions on Medical Imaging, vol. 33, no. 8, pp. 1702-1725, 2014.

[161] F. Shaffer and J. P. Ginsberg, "An overview of heart rate variability metrics and norms," Frontiers in Public Health, vol. 5, no. 258, 2017.

[162] J. R. Mitchell and J. Wang, "Expanding application of the wiggers diagram to teach cardiovascular physiology," Advances in Physiology Education, vol. 38, no. 2, pp. 170-175, 2014.

[163] M. Tavakoli, E. Kellar, D. Nassiri, and A. Joseph, "A novel polymeric coating for enhanced ultrasound visibility of medical devices," Medical device technology, vol. 17, no. 2, pp. 8-10, 12, 2006.

[164] C. R. Maurer, J. M. Fitzpatrick, M. Y. Wang, R. L. Galloway, R. J. Maciunas, and G. S. Allen, "Registration of head volume images using implantable fiducial markers," IEEE Transactions on Medical Imaging, vol. 16, no. 4, pp. 447-462, 1997.

[165] K. Kitamura, H. Shirato, S. Shimizu, N. Shinohara, T. Harabayashi, T. Shimizu, Y. Kodama, H. Endo, R. Onimaru, S. Nishioka, H. Aoyama, K. Tsuchiya, and K. Miyasaka, "Registration accuracy and possible migration of internal fiducial gold marker implanted in prostate and liver treated with real-time tumor-tracking radiation therapy (rtrt)," Radiotherapy and Oncology, vol. 62, no. 3, pp. 275-281, 2002.

[166] C. Pacchierotti, M. Abayazid, S. Misra, and D. Prattichizzo, "Teleoperation of steerable flexible needles by combining kinesthetic and vibratory feedback," IEEE Transactions on Haptics, vol. 7, no. 4, pp. 551-556, 2014.

[167] C. R. Wagner, N. Stylopoulos, and R. D. Howe, "The role of force feedback in surgery: analysis of blunt dissection," in Proceedings of the International Symposium on Haptic Interfaces for Virtual Environment and Teleoperator Systems (HAPTICS), pp. 68-74, Washington, USA, March 2002.

[168] K. B. Reed, A. M. Okamura, and N. J. Cowan, "Modeling and control of needles with torsional friction," IEEE Transactions on Biomedical Engineering, vol. 56, no. 12, pp. 2905-2916, 2009. 
[169] J. Humphrey, "Review paper: Continuum biomechanics of soft biological tissues," Proceedings of the Royal Society of London. Series A: Mathematical, Physical and Engineering Sciences, vol. 459, no. 2029, pp. 3-46, 2003. 


\section{Acknowledgements}

With great joy and satisfaction, I completed this thesis. However, this achievement would not have been possible without the help from a great number of people. Therefore, I would like to take this opportunity to express my gratitude and appreciation.

First, I would like to thank my doctoral supervisor Prof. Sarthak Misra for offering me a research opportunity in the field of medical robotics. I remember the first time I provided a conference paper draft to you in order to receive feedback. I knew my experience in scientific writing was zero and I also knew my writing skills were not that good. But when I received your first feedback ... my thoughts were ... how am I going to fix this?! Well, after a dozen revisions, I felt like, this could be going somewhere. A dozen revisions more, I knew this is going somewhere. Thank you for helping me to put my research findings into actual readable literature and for showing me the ways of scientific research.

Furthermore, I would like to thank members of the scientific staff of the Robotics and Mechatronics (RAM) group and the Biomechanical Engineering (BW) group. Prof. Bart Koopman and Prof. Stefano Stramigioli for their support, advice and inspiration. Prof. Jan Grandjean for showing me the clinical setting and explaining to me the relevant aspects from the clinicians point of view. And not to be forgotten, your endless supply of medical equipment that helped my research in more ways than you can imagine. Dr. Ferdi van der Heijden for showing me the world of computer vision. Ir. Edsko Hekman for helping me with the complex mechanical issues I needed to solve.

Furthermore, I would like to thank Prof. Paul Breedveld and Ir. Awaz Ali from the University of Delft for the interesting discussions we had and the support you provided. I also would like to thank Dr. Ron Alterovitz from the University of North Carolina at Chapel Hill and Dr. Sachin Patil from the University of California at Berkeley for a very productive collaboration in needle steering research.

A sincere thanks to my colleagues. Alper, thank you for being a control theorist ;-) when I needed it. A special thanks to my paranymphs Stefan Groothuis and Roy Roesthuis. Stefan, although we didn't share any research topics, you advice has always been appreciated. Roy, you have been a great sparring partner for all my research problems. I am very thankful for that! I also would like to thank the colleagues I shared an office with. Due to the many moves (i.e., from Carré $3^{\text {rd }}$ floor to Carré $2^{\text {nd }}$ floor to Horst-ring west $1^{\text {st }}$ floor to Horst-ring west ground floor), it could be possible that I am forgetting someone. But here it goes, thank you Dian, Gert, Momen, Navid and Fouzia for your 
support, advice and the interesting discussion we had. Further, I would like to thank my colleagues Pedro, Stefano, Federico, Klaas, Jakub and Christoff for our talks, your assistance and support on many occasions. Federico and Klaas we had a great time in Singapore!

A special thanks to all the students I worked with during my Ph.D. I am very grateful for the contributions you provided. Tim Ellenbroek for the initial version of the RADS setup. Manon Tolhuisen and Myléne Jansen for gathering and analysing pre-operative patient data from the clinic. Data I used to evaluate my robotic steering methods. Hassna Irzan for her contribution to the beating heart setup.

I would also like to thank the secretaries and technicians of the RAM and BW groups for the help they provided during my Ph.D. Secretaries Jolanda, Sandra, Lianne and Jeanine for solving my administrative problems and a good talk! Technicians Gerben, Hennie, Marcel and Wouter for your technical advice and for helping me to get the parts I needed for my setup. Nikolai for keeping the SVN repositories up and running. Technicians Remi and Menno from the Electronic and Mechanical Support Division of the Delft University of Technology for the help I received with the Steward platform and fabrication of catheter components.

Furthermore, I would like to thank my friends for their support, joy and the good times we have. This includes numerous parties, weekends and motorcycle trips. Further, I would like to thank the friends I met at the University. Meisters, thank you for the interesting talks, beers and extensive Christmas dinner parties.

To my parents, thank you for supporting me all of those years! This would not have been possible without your support and stimulation throughout my entire education. From a diploma of the ROC Technical College to get a B.Eng. degree from the Saxion University of Applied Science to obtain a M.Sc. degree from the University of Twente and finally to acquire a doctoral degree. Further, I would like to thank my sister Marleen and my parents in law Jan and Berna for their support when it was needed.

Last but not least, I would like to thank my beloved wife, Loes, for being there every step of the way. Throughout my Ph.D. you have been very supportive, understanding and patient. I could always rely on your helping hand. And most importantly, you gave me a push in the right direction when I needed it the most. I am lucky to have you! Thank you for your love, support and patience.

Gustaaf J. Vrooijink

De Lutte, the Netherlands 


\section{About the author}

Gustaaf Johannes Vrooijink is born in Oldenzaal, the Netherlands, on the $29^{\text {th }}$ of January, 1986. He went to primary school "St. Plechelmusschool" in De Lutte, the Netherlands, and secondary school "Twents Carmellyceum" in Oldenzaal, the Netherlands. At young age he developed an interest in computers and electronics. Hence, in 2002, he started a technical education at the ROC van Twente and graduated in 2006. In 2006, he continued his education at the Saxion University of Applied Sciences where he re-

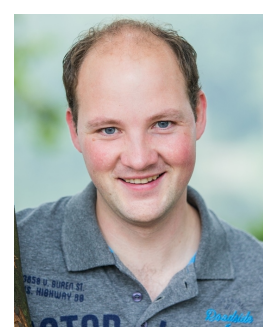
ceived his B.Eng. degree in Electrical Engineering in 2010. In addition to his education, he worked as a technician in the food processing industry, where he gathered practical experience in the field of electrical engineering. His deep interest in engineering motivated him to start at the University of Twente. He received his M.Sc. degree in electrical engineering from the University of Twente, Enschede, the Netherlands in 2012. He is currently pursuing the doctoral degree within the Department of Biomechanical Engineering, University of Twente, Enschede, the Netherlands. He is a member of the Surgical Robotics Laboratory (https://www.surgicalroboticslab.nl), University of Twente, where he was involved in the $i$ MIT-Instruments project for Minimally Invasive Techniques Interactive Multi-Interventional Tools (Project: MULTI)). His current research interests include medical robotics, control engineering, continuum robotics, computer vision using medical images and mimicking of a clinically-relevant environments for the evaluation of medical robotics. He is currently employed at Thales Nederland B.V. as a Systems Specialties Engineer. In his spare time he enjoys cycle racing and hiking. Furthermore, he likes to ride his motorcycle and spend time on home automation.

De Lutte, the Netherlands 